NIST Technical Note 1979

\title{
NIST Channel Sounder Overview and Channel Measurements in Manufacturing Facilities
}

\author{
Jeanne T. Quimby \\ Richard Candell \\ Kate A. Remley \\ David Novotny \\ Joseph Diener \\ Peter B. Papazian \\ Alexandra Curtin \\ Galen Koepke
}


NIST Technical Note 1979

\section{NIST Channel Sounder Overview and Channel Measurements in Manufacturing Facilities}

Jeanne T. Quimby ${ }^{1}$ Richard Candell ${ }^{2}$ Kate A. Remley ${ }^{1}$ David Novotny ${ }^{1}$ Joseph Diener ${ }^{3}$ Peter B. Papazian ${ }^{1}$ Alexandra Curtin ${ }^{1}$ Galen Koepke ${ }^{1}$

${ }^{1}$ Communication Technology Laboratory National Institute of Standards and Technology 325 Broadway, Boulder, CO 80305

${ }^{2}$ Engineering Laboratory National Institute of Standards and Technology 100 Bureau Drive, Gaithersburg, MD 20899

${ }^{3}$ Colorado School of Mines, Golden, Colorado

This publication is available free of charge from: https://doi.org/10.6028/NIST.TN.1979

November 2017

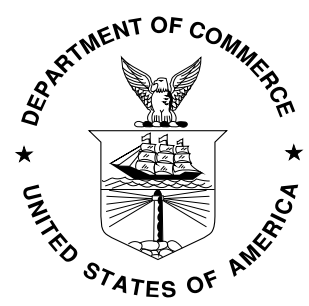

U.S. Department of Commerce Wilbur L. Ross, Jr., Secretary

National Institute of Standards and Technology Walter Copan, NIST Director and Undersecretary of Commerce for Standards and Technology 
Certain commercial entities, equipment, or materials may be identified in this document in order to describe an experimental procedure or concept adequately. Such identification is not intended to imply recommendation or endorsement by the National Institute of Standards and Technology, nor is it intended to imply that the entities, materials, or equipment are necessarily the best available for the purpose.

National Institute of Standards and Technology Technical Note 1979 Natl. Inst. Stand. Technol. Tech. Note 1979, 83 pages (November 2017) CODEN: NTNOEF

This publication is available free of charge from: https://doi.org/10.6028/NIST.TN.1979 


\section{Table of Contents}

1 Introduction - Purpose and Overview ……………..................................................... 9

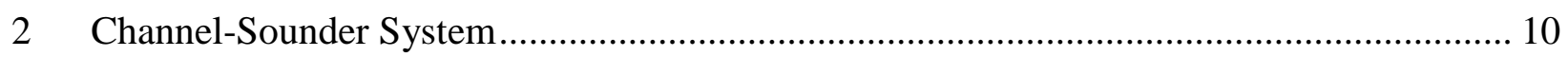

2.1 Channel-Sounder System Description .................................................................... 10

2.1.1 Channel-Sounder System Design Considerations ..................................................14

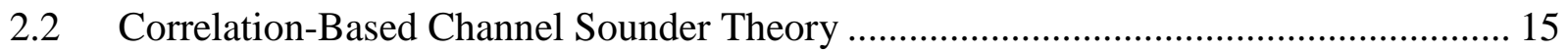

2.2.1 Correlation-based Channel Sounder Back-to-Back Calibration ...............................17

2.2.2 Channel Sounder Calibration Channel Response ......................................................18

2.2.3 Channel Sounder PDP and Path Gain ................................................................19

2.3 Channel-Sounder Measurement Approach ............................................................ 19

2.3.1 Channel-Sounder Measurement Approach for Mobile Measurements ....................20

2.3.2 Channel Sounder Measurement Approach for Fixed-Position Measurements........22

2.4 Channel Sounder Data Acquisition ........................................................................... 22

2.5 Channel Sounder Post-Processing............................................................................ 23

2.5.1 Correlation-Based Measurements Two-Slope Polynomial Fit .................................24

3 Channel-Sounder Configuration and Validation ................................................................. 25

3.1 Initial Set-up and Channel Sounder Calibration ......................................................... 25

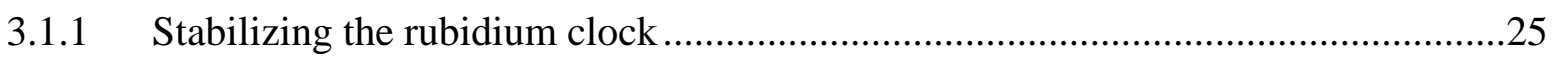

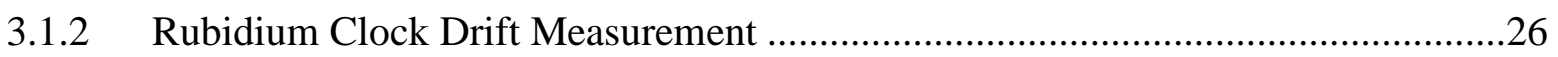

3.1.3 Power Amplifier Linearity Measurement .............................................................27

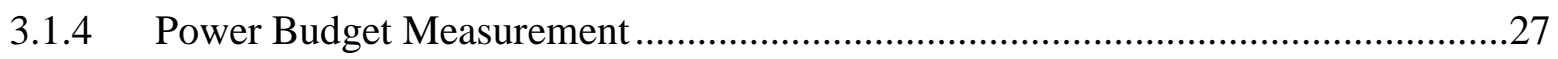

3.2 Open Area Test Site (OATS) Measurement Campaign .................................................... 29

4 Channel Measurements in Smart-Manufacturing Facilities ................................................... 32

$4.1 \quad$ NIST Central Utility Plant (CUP) Measurement Campaign ............................................ 32

4.2 Automotive Assembly Plant (AAP) Measurement Campaign......................................... 57

4.3 NIST-Gaithersburg, MD Machine Shop Measurement Campaign ................................... 65

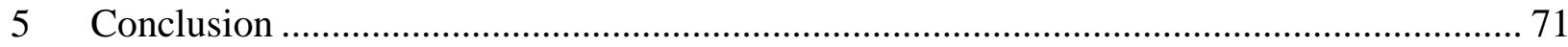

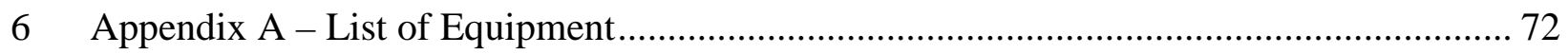

6.1 Transmitter (TX) System Equipment ....................................................................... 72

6.2 Receiver (RX) System Equipment ........................................................................... 72

6.3 Additional Channel Sounder System Equipment............................................................ 72

$7 \quad$ Appendix B - Installation Guide ................................................................................. 73

8 Appendix C - Channel Sounder Measurement Software Settings …………….................... 75 
9 Appendix D - Variability of Sounder Measurements in Manufacturing Facilities Guide.... 76

9.1 Variability in Path Loss Linear Regression.................................................................. 76

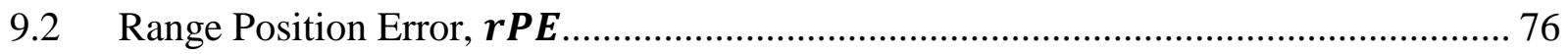

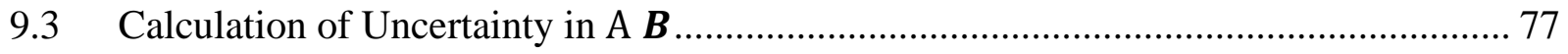

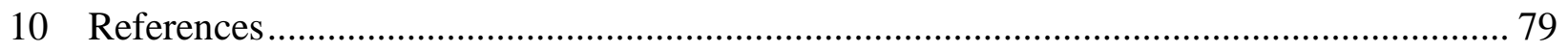




\section{List of Figures}

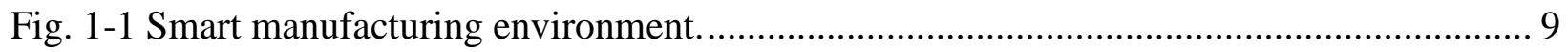

Fig. 1-2 Channel sounder system block diagram............................................................ 10

Fig. 2-1 Antenna gain for ETS-LINGRED 3115 Double-Ridged Guide antenna from ETS-

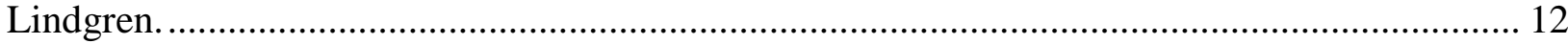

Fig. 2-2 TECOM omnidirectional biconical antenna gain and Seibersdorf Laboratories precision

omnidirectional dipole gain. Plot based on manufacturer data................................................ 12

Fig. 2-3 Antenna icon representation of the antennas........................................................... 13

Fig. 2-4 Hardware effects on the channel sounder measurement.......................................... 16

Fig. 2-5 Illustration of uncalibrated and linear-back-to-back-calibrated PDPs......................... 18

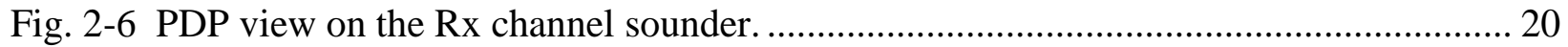

Fig. 2-7 Central Utility Plant (CUP) measurement route. ................................................... 21

Fig. 2-8 Example test sheet for acquisition number and check point linking............................ 21

Fig. 2-9 (a) RX antenna mounted on the antenna positioner for fixed measurements and (b)

antenna mount positions for check point 8 and a transmitter position of TX2 ......................... 22

Fig. 2-10 (a) Usable reference measurement for channel sounder post-processing, (b) unusable

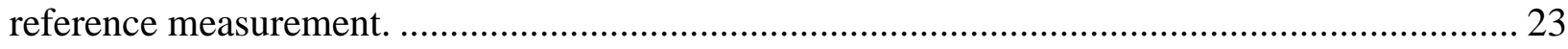

Fig. 2-11 (a) Reference measurement for $20 \mathrm{~dB}$ attenuation with $0 \mathrm{dBm}$ output power. (b)

Reference measurement for $50 \mathrm{~dB}$ attenuation with $0 \mathrm{dBm}$ output power.............................. 24

Fig. 3-1 Block diagram of the channel sounder architecture............................................... 25

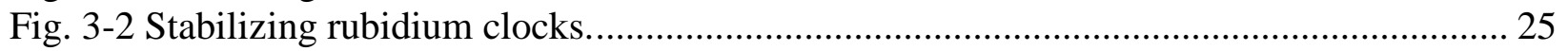

Fig. 3-3 Rubidium clocks drift measurement set-up........................................................... 26

Fig 3-4 (a) Clock discipline LabVIEW program with poor synchronization and (b) rubidium

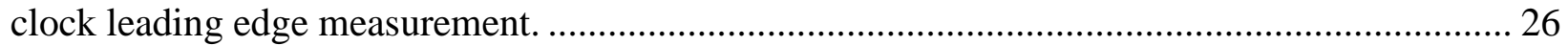

Fig. 3-5: Power budget measurement set-up...................................................................... 28

Fig. 3-6 Channel sounder deployment at NIST OATS facility. ............................................ 29

Fig. 3-7: Measurement of path gain set-up for the OATS .................................................... 29

Fig. 3-8 Path gain comparison to ray tracing at the OATS for (a) $2.245 \mathrm{GHz}$ and (b) $5.4 \mathrm{GHz}$. 31

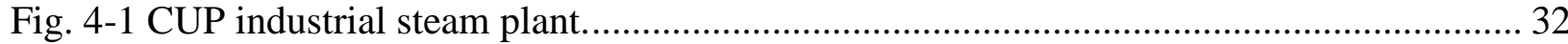

Fig. 4-2 Measurement route through the CUP facility. ........................................................ 33

Fig. 4-3 Path gain measurements of the NIST Central Utility Plant (CUP) for $2.245 \mathrm{GHz}$ (a)

Vpol omnidirectional TX antenna at position TX2 and Vpol omnidirectional RX antenna (b)

Raised Cosine filter applied to the TX signal for the same antenna configuration. .................... 38

Fig. 4-4 Path gain measurements of the NIST CUP for $2.245 \mathrm{GHz}$ Vpol horn TX antenna and

Vpol omnidirectional RX antenna at (a) position TX2 and (b) position TX3........................... 39

Fig. 4-5 Path gain measurements in the NIST CUP at $5.4 \mathrm{GHz}$ for TX antenna and Vpol or Xpol polarized RX omnidirectional antenna at (a) Vpol omnidirectional TX antenna position TX2 and

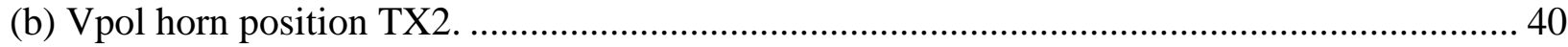

Fig. 4-6 Path gain measurements of NIST CUP at 5.4 GHz for TX antenna at position TX3 for

Vpol or Xpol omnidirectional RX antenna and (a) Vpol omnidirectional TX antenna and (b)

Vpol TX antenna........................................................................................................ 41

Fig. 4-7 Path gain measurements of NIST CUP at 2.245 GHz for Vpol TX antenna at position

TX2 or TX3 and Vpol omnidirectional RX antenna at check point 6: (a) range plot (b) contour

plot with omnidirectional TX antenna at check point 6 .

Fig. 4-8 Path gain measurements of NIST CUP at 2.245 GHz for Vpol horn TX antenna and

Vpol omnidirectional RX antenna at check point 6: (a) at position TX2, (b) at position TX3... 43 
Fig. 4-9 Path gain measurements of NIST CUP at 5.4 GHz for Vpol horn TX antenna at position TX3 and Vpol omnidirectional RX antenna at check point 6 (a) range plot (b) contour plot...... 44 Fig. 4-10 Path gain measurements of NIST CUP at 2.245 GHz for Vpol horn or omnidirectional TX antenna at position TX2 and Vpol omnidirectional RX antenna at check point 8: (a) range plot, (b) contour plot with omnidirectional TX antenna. ....................................................... 45 Fig. 4-11 Path gain measurements of NIST CUP at 2.245 GHz for Vpol TX antenna at position TX2 and Vpol omnidirectional RX antenna at check point 8: (a) omnidirectional TX antenna, (b) horn TX antenna.

Fig. 4-12 Path gain measurements of NIST CUP at 2.245 GHz for Vpol horn TX antenna at position TX3 and Vpol omnidirectional RX antenna at check point 8 (a) range plot (b) contour plot.

Fig. 4-13 Path gain measurements of NIST CUP at 2.245 GHz for Vpol horn or omnidirectional TX antenna at position TX2 and Vpol omnidirectional RX antenna at check point 13 (a) range plot (b) contour plot with Root Raised Cosine Filter applied to the TX signal with an omnidirectional TX antenna. 48

Fig. 4-14 Path gain measurements of NIST CUP at 2.245 GHz for Vpol horn TX antenna and Vpol omnidirectional RX antenna at check point 13: (a) contour plot at position TX2, (b) range plot at position TX3.

Fig. 4-15 Path gain measurements of NIST CUP for Vpol horn TX antenna and Vpol omnidirectional RX antenna at check point 13: (a) contour plot at $2.245 \mathrm{GHz}$, (b) range plot at $5.4 \mathrm{GHz}$.

Fig. 4-16 Path gain measurements of NIST CUP at 5.4 GHz for Vpol horn TX antenna and omnidirectional RX antenna: (a) contour plot for Vpol Rx antenna at check point 13, (b) range plot at $5.4 \mathrm{GHz}$ for Vpol or Xpol RX antenna at check point 18. ......................................... 51 Fig. 4-17 Path gain measurements of NIST CUP at 5.4 GHz for Vpol TX antenna at position TX3 and omnidirectional RX antenna at check point 18: (a) Vpol RX antenna, (b) Xpol RX antenna.

Fig. 4-18 Path gain measurements of NIST CUP at 5.4 GHz for Vpol horn TX antenna at position TX3 and omnidirectional RX antenna at check point 19: (a) range plot for Vpol or Xpol Rx antenna, (b) contour plot for Vpol RX antenna. 53 Fig. 4-19 Path gain measurements of NIST CUP at 5.4 GHz for Vpol horn TX antenna at position TX3 and omnidirectional RX antenna: (a) contour plot at check point 19 for Xpol RX antenna, (b) range plot at check point 29 for Vpol RX antenna. 54 Fig. 4-20 Path gain measurements of NIST CUP at 5.4 GHz for Vpol horn TX antenna at position TX3 and omnidirectional RX antenna at check point 29: (a) run 1, (b) run 2 .............. 55 Fig. 4-21 Path gain measurements of NIST CUP at 5.4 GHz for Vpol horn TX antenna at position TX3 and omnidirectional RX antenna at check point 33: (a) range plot, (b) contour plot.

Fig. 4-22 Measurement routes through the automotive assembly plant facility: (a) inner loop, and (b) outer loop.

Fig. 4-23 Path gain measurements of AAPlant for Vpol omnidirectional TX antenna at position TX1 for Vpol or Xpol omnidirectional RX antenna: (a) at $2.245 \mathrm{GHz}$ and (b) at $5.4 \mathrm{GHz}$. 61 Fig. 4-24 Path gain measurements of AAPlant for Vpol omnidirectional TX antenna at position TX1 for Vpol or Xpol omnidirectional RX antenna: (a) at 2.245 GHz internal run, and (b) at 5.4 $\mathrm{GHz}$ internal run. 
Fig. 4-25 Path gain measurements of AAPlant for Vpol omnidirectional TX antenna at position TX2 for Vpol or Xpol omnidirectional RX antenna: (a) at $2.245 \mathrm{GHz}$, and (b) at $5.4 \mathrm{GHz} . . . . . . .63$ Fig. 4-26 Path gain measurements of AAPlant for Vpol omnidirectional TX antenna at position TX2 for Vpol or Xpol omnidirectional RX antenna: (a) at $2.245 \mathrm{GHz}$ internal run, and (b) at 5.4

$\mathrm{GHz}$ internal run. 64

Fig. 4-27: Measurement route through the Gaithersburg machine shop. ….................................. 67 Fig. 4-28 Path gain measurements of Gaithersburg (GBurg) Machine Shop for Vpol omnidirectional TX antenna at position TX1 for Vpol or Xpol or Longpol omnidirectional RX antenna (a) at $2.245 \mathrm{GHz}$, and (b) at $5.4 \mathrm{GHz}$. 68 Fig. 4-29 Path gain measurements of Gaithersburg (GBurg) Machine Shop for Vpol omnidirectional TX antenna at position TX2low for Vpol or Xpol or Longpol omnidirectional RX antenna (a) at $2.245 \mathrm{GHz}$ and (b) at $5.4 \mathrm{GHz}$. 69 Fig. 4-30 Path gain measurements of Gaithersburg (GBurg) Machine Shop for Vpol omnidirectional TX antenna at position TX2high for Vpol or Xpol or Longpol omnidirectional

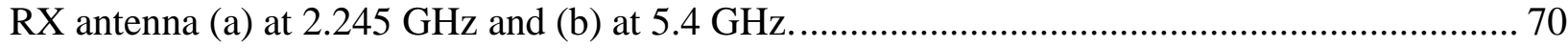

Fig. 7-1 Installation of PXIe-8135 controller into the PXIe-1085 receiver chassis....................... 73

Fig. 7-2 Installation of PXIe-5646R VST into the PXIe-1085 receiver chassis............................ 73

Fig. 7-3 Installation of PXIe-6683H and PXIe-6674T into the PXIe-1085 receiver chassis. ...... 73

Fig. 9-1 Path loss versus range with uncertainties for a human walking....................................... 78 


\section{List of Tables}

Table 2-1 Possible combinations of channel-sounder system parameters................................ 15

Table 3-1 OATS measurement campaign component losses .............................................. 27

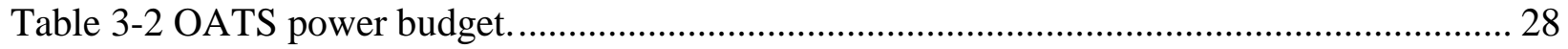

Table 4-1: CUP industrial steam plant TX locations............................................................. 32

Table 4-2: CUP mobile runs: omnidirectional = biconical antenna and horn = Double-Ridged

Guide antenna. 34

Table 4-3: CUP fixed-position runs: omnidirectional = biconical antenna and horn = Double-

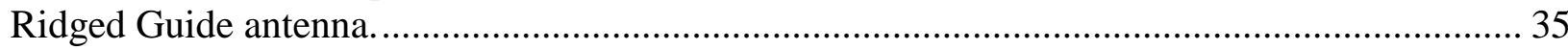

Table 4-4: Automotive Assembly Plant TX locations......................................................... 57

Table 4-5: AAP runs: omnidirectional = biconical antenna. .................................................. 58

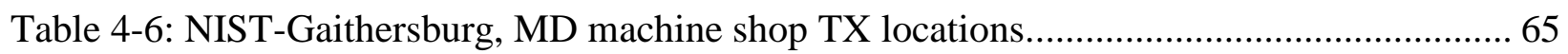

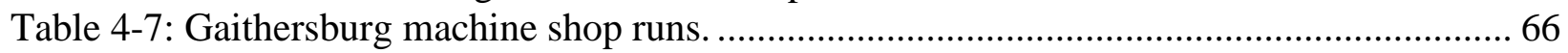

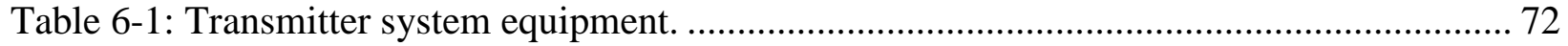

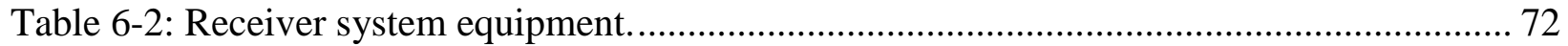

Table 6-3 Additional channel sounder system equipment..................................................... 72

Table 8-1 Set Time Reference LabVIEW code. ................................................................. 75

TABLE 8-2 Discipline clock LabVIEW code................................................................. 75

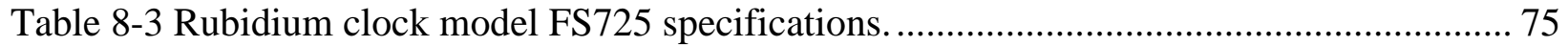

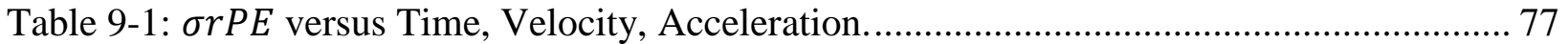

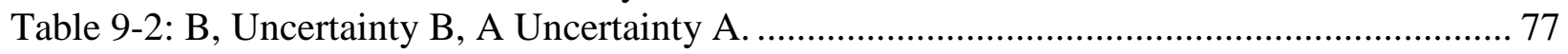




\section{Executive Summary}

Manufacturers are increasingly choosing wireless platforms to replace wired ones due to their ease of installation, upgrade, and reconfigurability. Reliable and secure real-time performance of wireless platforms is technically challenging because wireless data communication, reliability, and throughput can be easily affected by the physical environment in which the wireless platforms operate. These effects need to be investigated and better understood through physics-based modeling, correlated with real-world measurements.

To support U.S. manufacturers, the NIST Engineering Laboratory has launched a five-year project entitled "Wireless Systems for Industrial Environments" as part of the NIST Standards for Manufacturing Systems Program. The key idea for this project is to develop best-practice guidelines for integrated performance evaluation of secure wireless platforms in dynamic production systems. This will include a methodology for selecting wireless technology and protocols that will assure real-time performance of wireless platforms in various manufacturing environments. These guidelines will enable manufacturers, technology providers, and solution providers to design, deploy, and assess robust, secure integrated wireless solutions for use in smart manufacturing. As part of this project, NIST characterize the wireless environment through measurements in representative production facilities.

One objective for the wireless measurements in manufacturing environments presented here is to provide representative propagation-channel parameters for channel-model development. The measurements reported here have been carried out with a channel sounder that provides data on the attenuation and impulse response of the wireless channel. These "active measurements" were conducted in multiple locations within three manufacturing environments.

The measurement system, or "channel sounder", used for these measurements is described in detail in this technical note. The NIST channel sounder system is a Pseudo Noise (PN)-sequence correlation-based system with an operating frequency range of $65 \mathrm{MHz}$ to $6 \mathrm{GHz}$ and up to 200 $\mathrm{MHz}$ bandwidth. It consists of a single transmitter (TX) and a single receiver (RX) coordinated with two synchronized rubidium clock.

The channel sounder's transmitter repetitively transmits a PN sequence of digital symbols that modulate a Binary Phased-Shift Keying (BPSK) signal that is upconverted to the RF carrier frequency. After traveling through a power amplifier, the signal propagates over a RF channel to the channel sounder's receiver. The receiver downconverts and digitizes the received signal, which has been modified by channel impairments. Removal of the channel sounder hardware effects occur through use of back-to-back measurements.

Three industrial facilities were measured during our measurement campaigns. The NIST Central Utility Plant (CUP) is representative of a medium-sized steam plant. The CUP had few moving components during the measurement campaign that would produce fast fading except for the humans that were performing the measurements. Next, an Automotive Assembly Plant (AAP) had a considerable physical footprint with multiple moving components from inspection vehicles, 
gantries in the ceiling and humans performing tasks. Finally, the NIST Gaithersburg Machine Shop is a small machine shop at the NIST-Gaithersburg campus. It was a very static environment with very few moving parts during the measurement campaign. Specific dimensional information about the measurement campaigns environments are provided later. The measurement campaign data are available for download at http://doi.org/10.18434/T44S3N. Please reference the DOI when using the data for research and/or publications.

A brief summary analysis of the measurement campaign industrial environments:

Slopes derived from the linear regression for a large range values are comparable to previous studies for industrial facilities environments. The placement of the transmit antenna (with respect to its antenna pattern) is critical for nearby receiving sensors where the RF propagation has fewer multipath mechanisms to reach the receive antenna. The exact placement becomes less critical as the propagation path involves more multipath and utilizes fewer line-of-sight paths. The power received by the channel sounder was polarization dependent. 


\section{Introduction - Purpose and Overview}

The NIST Engineering Laboratory with support from NIST Communication Technology Laboratory (CTL) has launched a five-year project entitled "Wireless Systems for Industrial Environments” [1] as part of the NIST Standards for Manufacturing Systems Interactive Program. Manufacturers and manufacturing facilities are currently undergoing an incredible transformation. Along with the use of robotics to enable greater flexibility and efficiency on the factory floor, they are increasing their connectivity through use of wireless systems in place of wired systems. These wireless systems are easy to install, upgrade, and reconfigure on the factory floor. An example is shown in Fig. 1-1. Reliable and secure real-time performance of wireless systems is technically

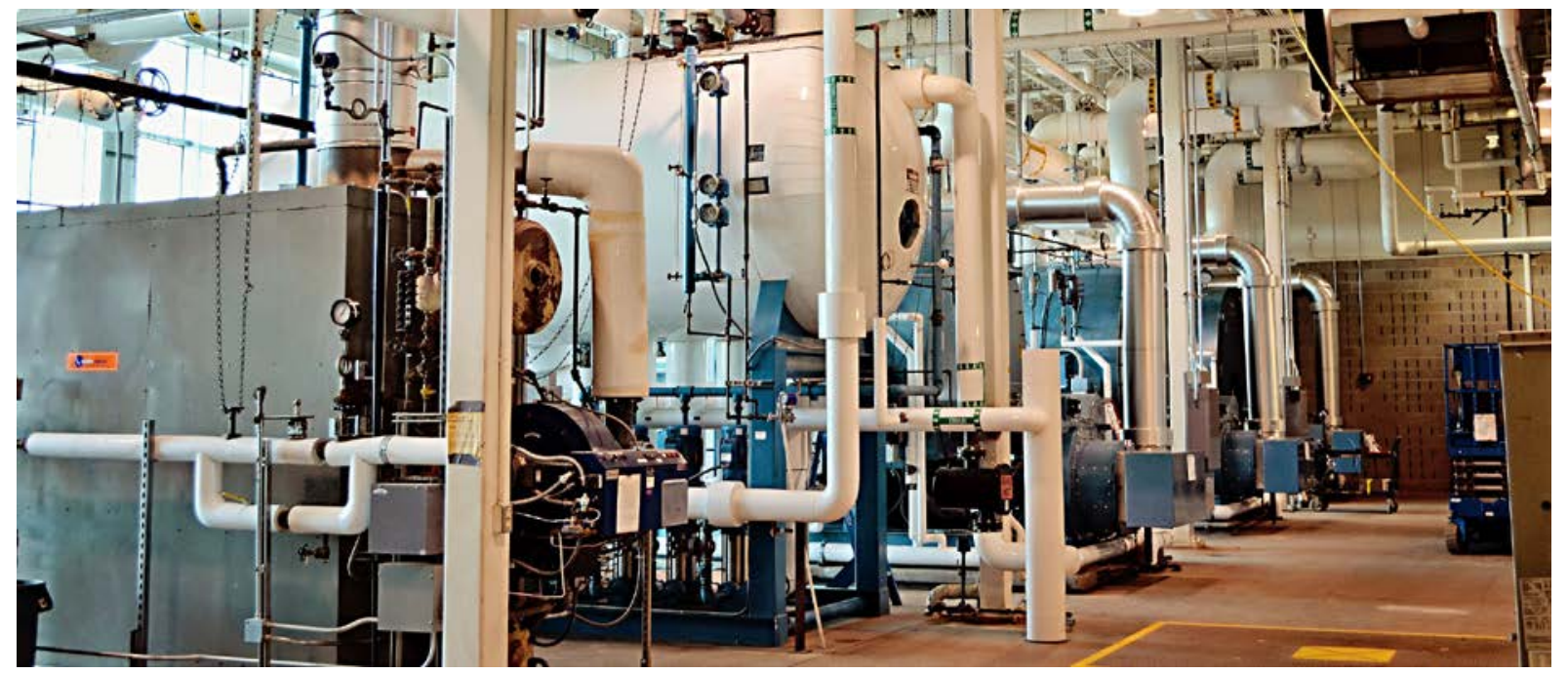

Fig. 1-1 Smart manufacturing environment.

challenging because wireless operation, reliability, and throughput can be easily affected by the physical environment. Investigation of these effects can be done through better understanding of wireless propagation if the models are correlated with real-world measurements of the wireless channel.

Real-world channel characterization of production environments is essential for deployment of reliable real-time wireless systems. Measurements and propagation characteristics of the wireless channel may be obtained with a channel sounder. The channel sounder collects impulse response data of both the spatial and temporal behavior of the wireless channel. This impulse response measures the electromagnetic environment of the channel by capturing the reflections, diffractions, and scattering phenomena that occur between the transmitter and receiver. This information can then help wireless systems and standards bodies by enabling the determination of data rate, InterSymbol Interference (ISI), and RMS delay spread, among many other useful parameters, when planning a suitable deployment of a system.

A straightforward way of performing channel sounding in the frequency domain is to use a vector network analyzer (VNA); however, this approach is typically too slow for these dynamic environments to capture the time-varying nature of a wireless channel across a wide bandwidth. VNAs are difficult to use when trying to capture the time-varying phenomena which arises when 

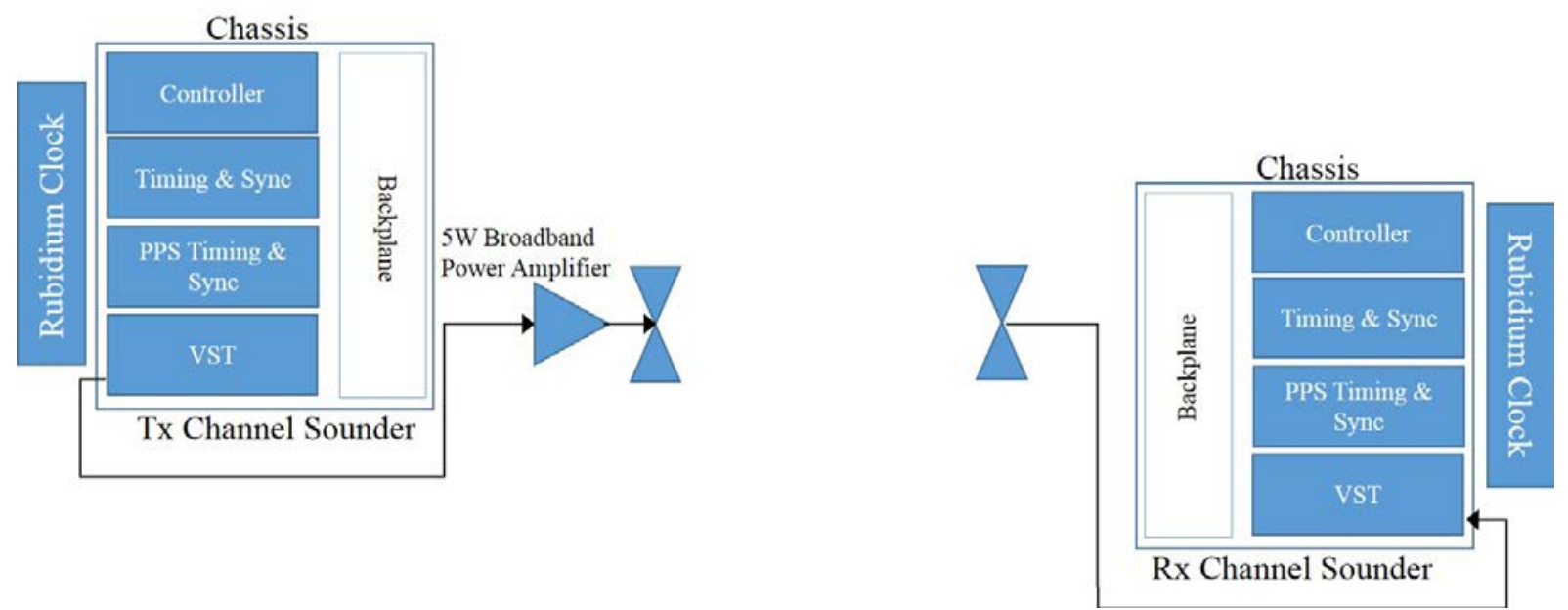

Fig. 1-2 Channel sounder system block diagram.

there is relative motion between the transmitter and receiver because the transmitter and receiver must be tethered together for timing synchronization.

To assess the dynamic characteristics of the channel, NIST used a correlation-based channel sounder. This channel sounder is mobile and utilizes rubidium clock to provide untethered synchronization. The sounder can make multiple measurements quickly for aggregation of data in a large wireless production environment. The channel sounder is capable of gathering data for carrier frequencies between $65 \mathrm{MHz}$ to $6 \mathrm{GHz}$ with an operational bandwidth up to $200 \mathrm{MHz}$ and a Vector Signal Transceiver (VST).

In the end, the measurements and propagation models developed for the correlation-based channel sounder system will help in developing best-practice guidelines for integrated performance evaluation of secure wireless platforms in dynamic production environments. This will include a methodology for selecting wireless technologies and protocols that will assure reliable real-time performance of wireless platforms in various manufacturing environments. These guidelines are intended to enable manufacturers, technology providers, and solution providers to design, deploy, and assess robust, secure integrated wireless solutions for use in smart manufacturing.

This technical note is organized as follows. The channel-sounder system is described in Section 2; channel sounder configuration hardware and system set-up for a measurement are given in Section 3; measurement campaigns with measured results are provided Section 4; and finally conclusions are drawn in Section 5.

\section{Channel-Sounder System}

\subsection{Channel-Sounder System Description}

The NIST channel-sounder system for the measurement of production environments is a PNsequence correlation-based system. It consists of a single transmitter (TX) and a single receiver (RX) synchronized with two rubidium clock, as shown in Fig. 1-2. The clock ensures that drift 
between samples is small enough for accurate delay resolution of the multipath components and also allows for measurement of the absolute timing between transmitter and receiver [2].

The channel sounder's transmitter contains a vector signal generator that generates a PN code sequence which modulates a Binary Phased-Shift Keying (BPSK) signal and upconverts to the RF carrier frequency. The bandwidth of the channel sounder corresponds to the bandwidth of the BPSK symbols modulated by the PN sequence. The vector signal generator is specified to have a maximum output power of $+10 \mathrm{dBm}$ with a $-161 \mathrm{dBm} / \mathrm{Hz}$ noise floor. The waveform corresponding to each PN sequence is oversampled by a factor of four, providing, 8188 samples with a 5 nanosecond/symbol sampling rate. Therefore, a single record of 400 PN sequences (or “code words") has a duration of 16.37 milliseconds.

The channel sounder's transmitter repetitively transmits a PN sequence of digital symbols (the PN code) with a maximum length sequence order of 11, depending upon the measurement campaign. The average power transmitted is maintained through the continuous transmission of the signal. The PN code is transmitted through an amplifier and a matched filter to reduce the radiated harmonics. The signal is then transmitted to the receiver either through an attenuator for a backto-back measurement or over an RF propagation channel measurement.

The channel sounder's receiver downconverts and digitizes the received signal, which has been modified by channel impairments. Correlation processing of the measured signal is performed in post processing to obtain the channel's time-domain impulse response. This system exploits correlation processing gain proportional to the maximum length sequence order while suppressing narrowband interference signals. From the time-domain impulse response, the Power Delay Profile (PDP) can be computed along with other channel parameters such as RMS delay spread, number of multipath components, and initial time of arrival. The channel sounder has a processing gain that is proportional to the length of the PN length. With the processing gain, this channel sounder achieves higher dynamic range than a simple wideband measurement system. Our system uses National Instruments ${ }^{1}$ hardware and software for data acquisition, with NIST-written programs used for post processing.

The timing system is crucial for the synchronization between the transmit and receive channel sounder sections when they are disconnected from one another. The transmitter system is tied directly to a $10 \mathrm{MHz}$ rubidium clock with a timing synchronizer (NI PXI 6674T). This unit shares clocks and triggers between the multiple modules in the chassis (see Fig. 1-2). A timing synchronization unit (NI PXI 6683H) generates the triggers from the Pulse Per Second (PPS) signal of the rubidium clock and disciplines its Temperature Compensated Crystal Oscillator (TCXO). The rubidium clock's PPS is used to create a reference trigger to coherently initiate signal generation in the transmitter and acquisition in the receiver. With this approach, the trigger timing and the Local Oscillator (LO) RF up- and down- conversion are locked and synchronized. This use of the rubidium clock for both triggering and frequency conversion from the LO minimizes the distribution jitter and minimizes phase and time drift.

${ }^{1}$ The mention of brand names does not imply an endorsement by NIST. Other products may work as well or better. 
The RF section of the transmitter is incorporated in the vector signal generator. The output of the vector signal generator was connected to an amplifier. For the results presented here, the amplifier setting was adjusted so that the input power into the antenna was 1.5 watts at the $2.245 \mathrm{GHz}$ operating frequency and 1.25 watts at the $5.4 \mathrm{GHz}$ operating frequency.

The transmit antenna used was either a TECOM [3] broadband, omnidirectional, slant linear, biconical antenna or an ETS-LINGREN 3115 double-ridged guide antenna [4]. The nominal vertical plane beamwidth for the TECOM antenna is from $100^{\circ}$ to $35^{\circ}$ [3] over the operating frequency of 1.0 to $4.0 \mathrm{GHz}$. The nominal antenna gain $g^{\mathrm{TX}}$ is $2.9 \mathrm{dBi}$ at the $2.245 \mathrm{GHz}$ operating frequency and $3.6 \mathrm{dBi}$ at the $5.4 \mathrm{GHz}$ operating frequency, as seen in Fig. 2-2. The antenna gain for the ETS-LINGREN 3115 double-ridged guide is around $9.26 \mathrm{dBi}$ for the $2.245 \mathrm{GHz}$ operating frequency and $10.32 \mathrm{dBi}$ for the $5.4 \mathrm{GHz}$ operating frequency as seen in Fig. 2-1. The entire transmitter system except for the monitor, keyboard, power amplifier, and antenna was contained in a single chassis.

The receiver is based upon a vector signal analyzer. The vector signal analyzer demodulates the BPSK signal from the impaired channel to measure signal $S_{\text {meas }}$. As with the $\mathrm{TX}$, the timing system for the RX is tied directly to a $10 \mathrm{MHz}$ rubidium clock using a timing router. The RX has an internal computer to run the LabVIEW software and to save data to an NI HDD-8260 Redundant Array of Independent Disks (RAID) hard drive, a data storage unit located in the RX chassis.

The receive antenna was a Seibersdorf Laboratories Precision Omnidirectional Dipole [5]. The antenna gain is $-4.2 \mathrm{dBi}$ at the 2.245 $\mathrm{GHz}$ operating frequency and $-3.5 \mathrm{dBi}$ at the 5.4 $\mathrm{GHz}$ operating frequency as seen in Fig. 2-2. The antenna icon representation may be seen in Fig. 2-3. These icons represent the antenna type and antenna orientation used during the measurement campaigns shown in Section 4.

We automatically save the collected data are automatically saved onto a RAID drive using the NI proprietary "tdms" file type specifically designed for waveform data. We collected data extracted from this tiered file type using a MathWorks MATLAB program written by NIST staff. The .tdms file bins the measured

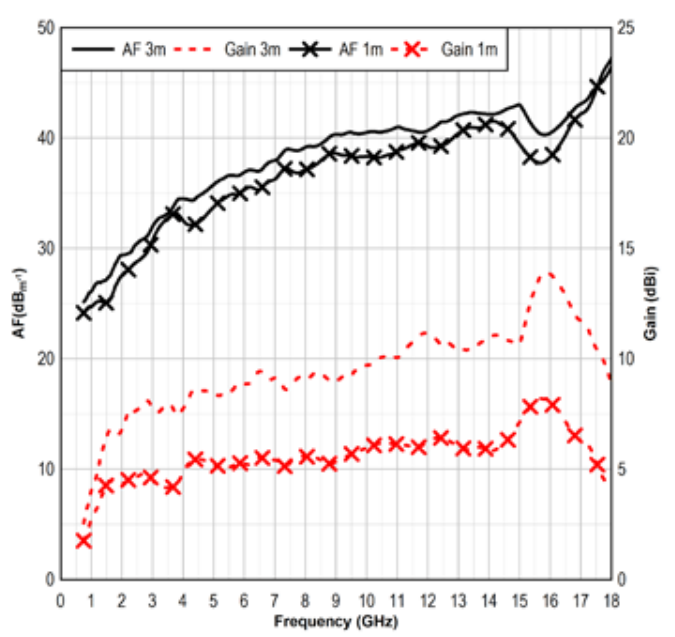

Fig. 2-1 Antenna gain for ETS-LINGRED 3115 double-ridged guide antenna from ETS-Lindgren.

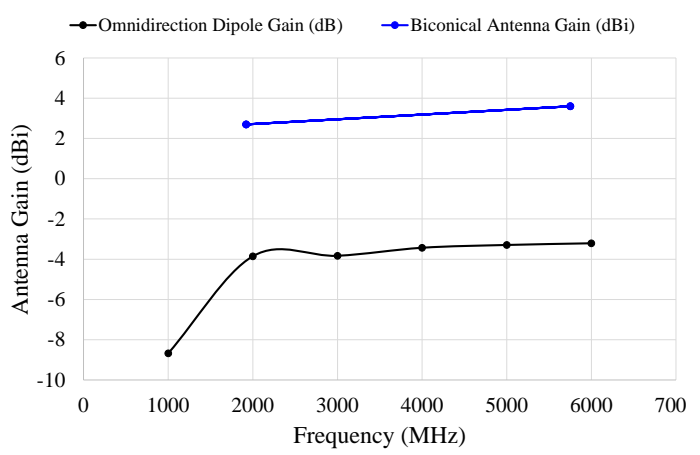

Fig. 2-2 TECOM omnidirectional biconical antenna gain and Seibersdorf Laboratories precision omnidirectional dipole gain. Plot based on manufacturer data. 


\section{ETS-LINGREN 3115}

Double-Ridged Guide antenna

TECOM broadband, omnidirectional, slant linear biconical antenna Seibersdorf Laboratories Precision Omnidirectional Dipole

Fig. 2-3 Antenna icon representation of the antennas. channel-sounder impulse responses into "records." The length of a record equals the length of the ideal $\mathrm{PN}$ sequence, $\mathrm{PN}_{\text {ideal, }}$ times the number of samples/symbol (e.g., a 2047-point ideal PN sequence times four samples/symbol equals 8188). We term a collection of records an "acquisition." The number of records in an acquisition is userspecified in the TX control software. A "file" is made up of user-defined number of acquisitions. Multiple files may be created during a measurement run. The total number of acquisitions

in a measurement run equals the sum of the acquisition files for a particular measurement run. A specific acquisition is denoted by its acquisition number within the measurement run as defined by its sequential-order number within the filename. For example, the tenth acquisition is 10 . Using a NIST-written MATLAB program, we can process the collected data to obtain the I/Q data of the channel measurement. A description of the measurement approach and post processing algorithm are provided later in this document.

Finally, the acquisition time is related to the time duration of a single record, the number of records, and the number of acquisitions for a single data run. A record of the timing data is contained in each .tdms file. Using information contained in these files, we can determine information about the start of a record or acquisition. Using the symbol period $T_{\text {sym }}$ with the duration of a code word $T_{c w}$, and the number of samples per record $S_{r e c}$, we can determine the duration of a single record, $T_{\text {rec }}$. $T_{\text {sym }}$ may be computed from the inverse of the Symbol Rate $\mathrm{R}_{\text {sym }}$, which is the number of symbols transmitted per second. In the case of oversampling, the Sample Rate $R_{S}$ is very useful. The $R_{S}$ equals $\frac{\text { samples }}{\text { symbol }}$, corresponding to the number of IQ samples in 1 symbol multiplied by the $\mathrm{R}_{\text {sym }}$. Key relationships between these quantities are given in (2.1) - (2.6).

$$
\begin{gathered}
T_{\text {sym }}=\frac{1}{\mathrm{R}_{\text {sym }}} \\
R_{S}=\mathrm{R}_{\text {sym }} \frac{\text { Samples }}{\text { Symbols }} \\
\text { Samples }_{\text {codeword }}=\left(2^{N}-1\right) \frac{\text { Samples }}{\text { Symbol }} \\
T_{c w}=\frac{\text { Samples }}{R_{S}} \\
T_{r e c}=T_{c w} N_{c w}=\frac{\left(2^{N}-1\right)}{R_{S}} N_{c w}
\end{gathered}
$$


where $N$ is the maximum length sequence orde, and $N_{c w}$ is the number of codewords for a single record. Therefore, if the $\mathrm{R}_{\text {sym }}=50$ Msymbols/second, $N_{c w}=400, \mathrm{~N}=2047$, $\frac{\text { Samples }}{\text { symbol }}=4$, then $T_{\text {rec }}$ equals 16.37 milliseconds. If there are 40 records per acquisition and 60 acquisition per .tdms file, we can calculate the total time for a single .tdms file as 39.3 seconds, using Eq. 2.5. We add a 2 millisecond delay between acquisitions to obtain the duration of a file

$$
T_{\text {file }}=\left(T_{\text {rec }} N_{\text {records/acqusition }}+2 \text { millisecond }\right) * N_{\text {acqusition } / \text { tdms }} .
$$

\subsubsection{Channel-Sounder System Design Considerations}

The channel sounder's delay resolution, detectable distance, dynamic range and maximum Doppler shift are important design considerations. The resolution that the channel sounder can obtain for closely spaced multipath components is a function of the Symbol Rate, $\mathrm{R}_{\text {sym }}$. The delay resolution, $\Delta T$ corresponds to the $3 \mathrm{~dB}$ bandwidth for the correlation pulse

$$
\Delta T=\frac{2}{R_{S}} \text {. }
$$

This time resolution is the accuracy of determining the relative delay of a given multipath component. The maximum detectable delay can be computed from the delay resolution with

$$
T_{\max }=\left(2^{N}-1\right) T_{\text {sym }} .
$$

We can compute the maximum detectable distance $D_{\max }$ from the multipath time resolution using

$$
D_{\max }=c T_{\max },
$$

where $c$ is the speed of light, $c=2.99792 \times 10^{8} \mathrm{~m} / \mathrm{s}$.

The dynamic range of the channel sounder determines how accurately both strong and weak multipath components can be measured. The dynamic range will be limited by the noise floor of the hardware instrumentation but acquires additional range using the correlation processing gain from the PN sequence. The correlation processing gain, $P G_{\mathrm{dB}}$, may be computed from

$$
P G_{d B}=10 \log _{10}\left(2^{N}-1\right) .
$$

The maximum Doppler shift $v_{\max }$ can be measured at the rate that the channel measurements are made [6, 7]

$$
v_{\text {max }}=\frac{1}{2 * T_{\text {rec }}} .
$$

Therefore, increasing the PN sequence length, which is advantageous for dynamic range and maximum detectable delay, increases the symbol period while decreasing Doppler shift resolution. Table 2-1 shows some typical channel-sounder system parameters for the system described here for different frequencies with $N_{c w}=400$. 
Table 2-1 Possible combinations of channel-sounder system parameters.

\begin{tabular}{|c|c|c|c|c|c|c|c|}
\hline \multicolumn{3}{|c|}{$\begin{array}{c}\text { Users Inputs to TX Control } \\
\text { Software }\end{array}$} & \multicolumn{5}{|c|}{ Derived Channel Measurement Parameters } \\
\hline $\mathbf{N}$ & $\underset{(\mathbf{M S y m} / \mathrm{S})}{\boldsymbol{R}_{\boldsymbol{S v m}}}$ & $\begin{array}{c}\text { Sampl } \\
\text { es/Sym } \\
\text { bol }\end{array}$ & $\begin{array}{c}\Delta T \text { (ns) } \\
\text { (Eq. 2.7) }\end{array}$ & $\begin{array}{r}T_{\max }(\mu \mathrm{s}) \\
(\mathrm{Eq} .2 .8)\end{array}$ & $\begin{array}{r}D_{\max }(\mathrm{km}) \\
\quad \text { (Eq. 2.9) }\end{array}$ & $\begin{array}{c}\text { PG(dB) } \\
\text { (Eq. 2.10) }\end{array}$ & $\begin{array}{r}v_{\max }(\mathrm{Hz}) \\
(\mathrm{Eq} .2 .11)\end{array}$ \\
\hline 11 & 20 & 4 & 25 & 102.3 & 304.8 & 67 & 12.2 \\
\hline 11 & 40 & 4 & 12.5 & 51.2 & 152.5 & 67 & 24.4 \\
\hline 11 & 50 & 4 & 10 & 40.9 & 121.8 & 67 & 30.5 \\
\hline 11 & 100 & 2 & 10 & 20.5 & 61.1 & 67 & 61.1 \\
\hline 12 & 20 & 4 & 25 & 102.3 & 304.8 & 72 & 6.1 \\
\hline 12 & 40 & 4 & 12.5 & 51.2 & 152.5 & 72 & 12.2 \\
\hline 12 & 50 & 4 & 10 & 40.9 & 121.8 & 72 & 15.3 \\
\hline 12 & 100 & 2 & 10 & 20.5 & 61.1 & 72 & 30.5 \\
\hline
\end{tabular}

\subsection{Correlation-Based Channel Sounder Theory}

The channel impulse response (CIR) is a valuable parameter for channel sounder measurements and analysis [8]. The CIR may be derived from $h(\tau)$, the impulse response of the wireless channel such as the industrial environment between transmit and receive antennas defined as

$$
\hat{h}(\tau)=g^{\mathrm{TX}}(\tau) * h(\tau) * g^{\mathrm{RX}}(\tau),
$$

where * denotes temporal convolution. The antenna gain patterns for the transmitter and receiver are $g^{\mathrm{TX}}(\tau)$ and $g^{\mathrm{RX}}(\tau)$, respectively for a given elevation and azimuthal angle.

Collecting data in the industrial environments with the correlation-based channel sounder requires multiple hardware components and processing.; these hardware components such as the antennas and the non-ideal effects of the transmitter and receiver can have adverse effects on the impulse channel response of the environment. The non-ideal effects can come from the Digital-Analog Converters (DAC), Analog-Digital Converters (ADC) the frequency upconverters/downconverters mixers, power amplifiers, and many other hardware components.

These effects must be removed from the measurements $S_{\text {meas }}$ prior to calculation of channel sounder parameters such as PDP, RMS delay spread, and others channel sounder quantities. The correlation-based channel sounder setup for measurement of hardware effects is shown in Fig. 2-4. 


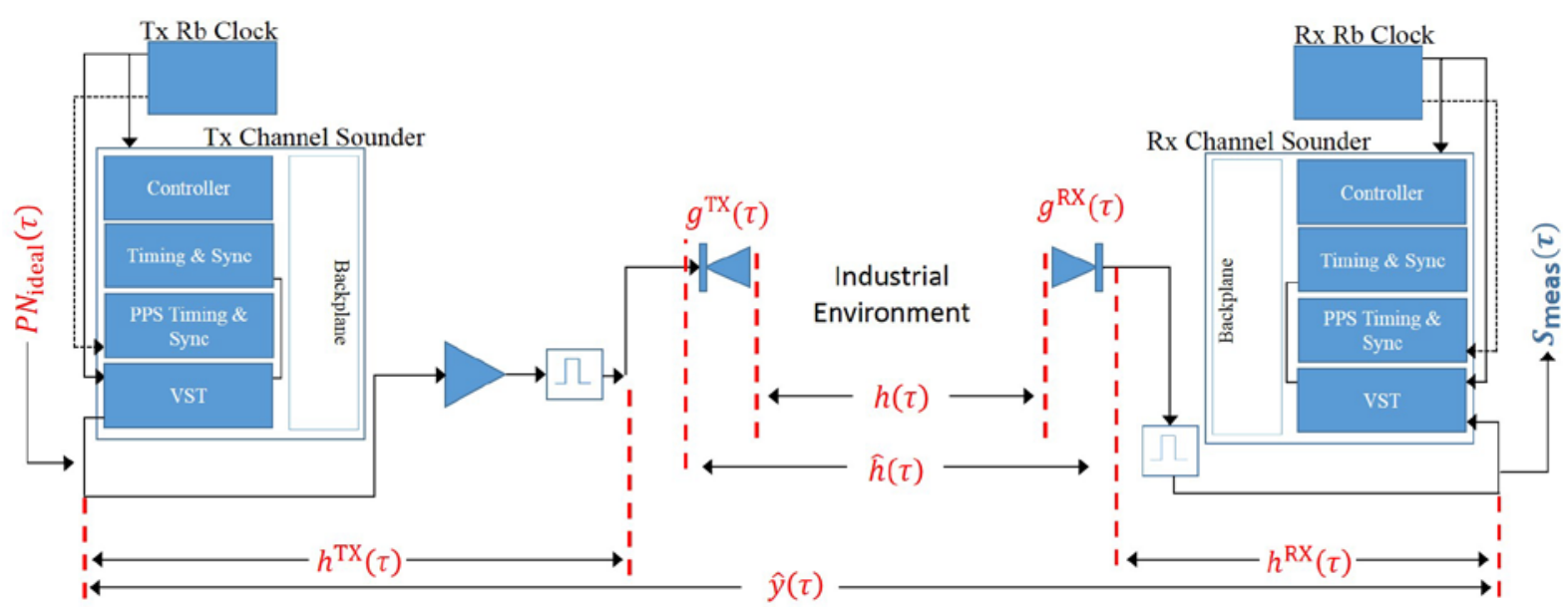

Fig. 2-4 Hardware effects on the channel sounder measurement.

As described in Section 2.1, the transmitter channel sounder repetitively transmits the $P N_{\text {ideal }}(\tau)$. After propagating through the $\mathrm{Tx}$ channel sounder non-ideal hardware responses $h^{\mathrm{TX}}(\tau)$, the industrial environment $h(\tau)$, the $\mathrm{Rx}$ channel sounder non-ideal channel sounder hardware responses $h^{\mathrm{RX}}(\tau)$, the signal is measured by the Rx channel sounder as $S_{\text {meas }}(\tau)$, as seen in Eq. 2.13:

$$
S_{\text {meas }}(\tau)=P N_{\text {ideal }}(\tau) * h^{\mathrm{TX}}(\tau) * g^{\mathrm{TX}}(\tau) * h(\tau) * g^{\mathrm{RX}}(\tau) * h^{\mathrm{RX}}(\tau) .
$$

An estimate of the CIR is computed by the convolution of the measured signal with the ideal PN sequence. The uncalibrated and unfiltered CIR $\hat{y}(\tau)$ is

$$
\hat{y}(\tau)=S_{\text {meas }}(\tau) * P N_{\text {ideal }}(-\tau) .
$$

If we rewrite Eq. 2.13 in terms of $\hat{y}(\tau)$ and using $\hat{h}(\tau)=g^{\mathrm{TX}}(\tau) * h(\tau) * g^{\mathrm{RX}}(\tau)$ leads to

$$
\hat{y}(\tau)=P N_{\text {ideal }}(\tau) * h^{\mathrm{TX}}(\tau) * \hat{h}(\tau) * h^{\mathrm{RX}}(\tau) * P N_{\text {ideal }}(-\tau) .
$$

The autocorrelation $p(\tau)$ of the ideal PN sequence $p(\tau)=P N_{\text {ideal }}(\tau) * P N_{\text {ideal }}(-\tau)$. Applying the associative property to the uncalibrated and unfiltered CIR, $\hat{y}(\tau)$ becomes

$$
\hat{y}(\tau)=p(\tau) * h^{\mathrm{TX}}(\tau) * \hat{h}(\tau) * h^{\mathrm{RX}}(\tau), \text { where }
$$

$p(\tau)$ is usually a short, impulsive-like signal that may be several time steps in duration and has a frequency response of

$$
P(f)=\mathcal{F}[p(\tau)]=\mathcal{F}\left[P N_{\text {ideal }}(\tau)\right] \times \mathcal{F}^{*}\left[P N_{\text {ideal }}(\tau)\right],
$$

where $\mathcal{F}$ is the Fourier transform and the superscript * denotes the complex conjugate. 


\subsubsection{Correlation-based Channel Sounder Back-to-Back Calibration}

The correlation-based channel sounder used a "back-to-back" measurement to correct for the linear distortion introduced by non-ideal system hardware. The TX channel sounder and RX channel sounder were connected via $50 \mathrm{~dB}$ of attenuator and RF coaxial cables without the antennas. The frequency response of the attenuations and cables were measured by a VNA. The constant attenuation, $A$, where $A=10^{\text {Attenuation }(\mathrm{dB}) / 20}$, is a function of frequency.

The back-to-back signal $y^{\mathrm{B} 2 \mathrm{~B}}(\tau)$ is

$$
y^{\mathrm{B} 2 \mathrm{~B}}(\tau)=A \times p(\tau) * h^{\mathrm{TX}}(\tau) * h^{\mathrm{RX}}(\tau) .
$$

To obtain the calibrated the CIR, $y^{\mathrm{B} 2 \mathrm{~B}}(\tau)$ is deconvolved from $\hat{y}(\tau)$ within the band of interest; thus, we remove the non-ideal effects of $h^{\mathrm{TX}}(\tau)$ and $h^{\mathrm{RX}}(\tau)$ :

$$
\begin{gathered}
y(\tau)=\mathcal{F}^{-1}\left\{\mathcal{F}[w(\tau)] \times \frac{\mathcal{F}\left[S_{\text {meas }}(\tau) * P N_{\text {ideal }}(-\tau)\right]}{\mathcal{F}\left[S_{\text {meas }}^{\text {B2B }}(\tau) * P N_{\text {ideal }}(-\tau)\right] / \mathcal{F}[A]}\right\} \\
=\mathcal{F}^{-1}\left\{\mathcal{F}[w(\tau)] \times \frac{\mathcal{F}\left[S_{\text {meas }}(\tau)\right] \times \mathcal{F}\left[P N_{\text {ideal }}(-\tau)\right]}{\mathcal{F}\left[S_{\text {meas }}^{\text {B2B }}(\tau)\right] \times \mathcal{F}\left[P N_{\text {ideal }}(-\tau)\right] / \mathcal{F}[A]}\right\},
\end{gathered}
$$

where $y(\tau)$ is the calibrated and filtered CIR, $\mathcal{F}^{-1}$ denotes the inverse Fourier transform, $S_{\text {meas }}^{\mathrm{B} 2 \mathrm{~B}}(\tau)$ is the back-to-back-measured signal, and $w(\tau)$ is a bandpass filter (or "window" in the frequency domain).

Eq. 2.19c is used for all processing of the PDPs. At the band edges in Eq. 2.19c, the noise in $\mathcal{F}\left[S_{\text {meas }}(\tau)\right]$ is divided by the noise in $\mathcal{F}\left[S_{\text {meas }}^{\text {B2B }}(\tau)\right]$. Because the measured quantities contain noise, dividing them typically will not result in a numerical division by zero resulting in a software anomaly. However, division of the low-level noisy signals can amplify the noise at the band edges. The bandpass filter $w(\tau)$ is often used to remove this amplified noise from $y(\tau)$ and to reduce sidelobes by truncating the measured spectrum.

As illustrated in Fig. 2-5, the linear-back-to-back-calibrated PDP (blue curve) removes a number of the non-ideal effects (seen in the tails after the main peak) in the channel-sounding system hardware. The blue curve represents calibrated, filtered, and discrete sum scaled PDP. The green curve is the uncalibrated PDP and it is scaled to match the calibrated PDP. 


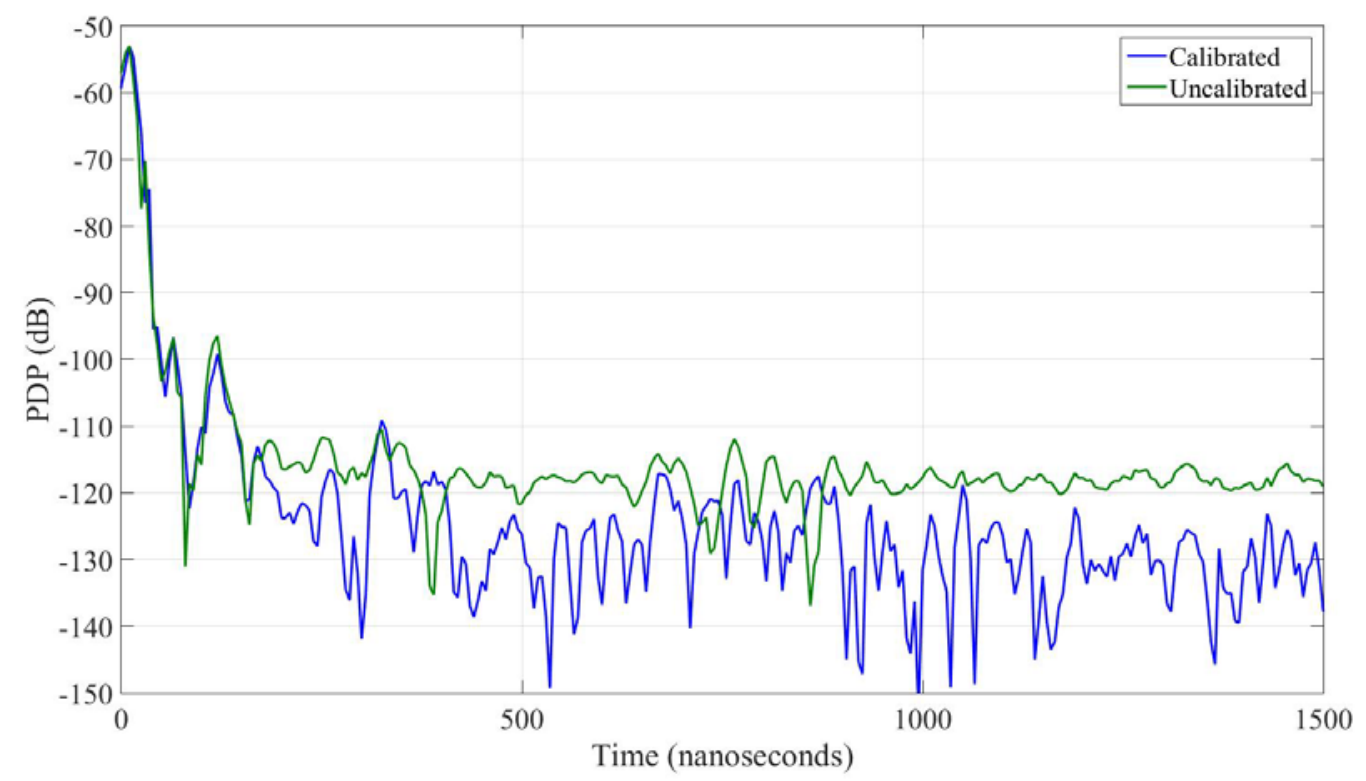

Fig. 2-5 Illustration of uncalibrated and linear-back-to-back-calibrated PDPs.

\subsubsection{Channel Sounder Calibration Channel Response}

$y(\tau)$ is the calibrated and filtered version of uncalibrated channel response, $\hat{y}(\tau)$.

$$
y(\tau)=\mathcal{F}^{-1}\left\{\mathcal{F}[w(\tau)] \times \frac{\mathcal{F}\left[S_{\text {meas }}(\tau)\right]}{\mathcal{F}\left[S_{\text {meas }}^{\text {BRe }}(\tau)\right] / \mathcal{F}[A]}\right\} .
$$

However, keep in mind that $y(\tau)$ is only an estimate of the channel response. This is true, not only because of the presence of noise, but also because of the additional filtering imposed on $y(\tau)$ by the filter $w(\tau)$.

One common implementation of $w(\tau)$ comes from the $P N_{\text {ideal }}$ code as seen in Eq. 2.20. Here, the $P N_{\text {ideal }}(\tau)$ is the oversampled PN sequence used by the transmitter where $N$ is the maximum length sequence order and divided by $2 \times \sqrt{N}$ to obtain unity gain for $w(\tau)$ :

$$
w(\tau)=\mathcal{F}^{-1}\left\{\mathcal{F}\left[\frac{P N_{\text {ideal }}(\tau)}{2 \times \sqrt{N}}\right] \times \mathcal{F}^{*}\left[\frac{P N_{\text {ideal }}(\tau)}{2 \times \sqrt{N}}\right]\right\} .
$$

\subsubsection{Channel Sounder Scaling and Calibration of Channel Response}

A reduction of the magnitude of the received signal occurs when the filter $w(\tau)$ is applied. To rescale the magnitude of the $y(\tau)$, we applied a Discrete Sum scaling [9] to achieve a calibrated and scaled solution, $y_{\text {scaled }}(\tau)$. Using Eq. 2.25, we implemented the scaling as 


$$
y_{\text {scaled }}(\tau)=y(\tau) \times \frac{\sqrt{N}}{\sqrt{\sum_{n=1}^{N_{f}}|\mathcal{F}[w(\tau)]|^{2}}} .
$$

The summation in Eq. 2.22 is over the frequency components, which results in a scalar value. This value may be taken in or out of the Fourier transform depending upon the desired implementation of the scaling.

\subsubsection{Channel Sounder PDP and Path Gain}

The average path gain over the frequency range may be computed from the calibrated and scaled channel response. Often, the PDP is used for this purpose and can be computed from the $y_{\text {scaled }}(t)$ in the time domain as

$$
\begin{gathered}
P D P(\tau)=\left|y_{\text {scaled }}(\tau)\right|^{2}, \\
P D P(\tau)=\frac{N}{\sum_{n=1}^{N}|\mathcal{F}[w(\tau)]|^{2}}\left|\mathcal{F}^{-1}\left\{\frac{\mathcal{F}[w(\tau)] \times \mathcal{F}\left[S_{\text {meas }}(\tau)\right]}{\mathcal{F}\left[S_{\text {meas }}^{\mathrm{B} 2 \mathrm{~B}}(\tau)\right] / \mathcal{F}[A]}\right\}\right|^{2} .
\end{gathered}
$$

While the channel path gain $G$ can be computed in the time domain, we can also compute it by averaging over the frequency-domain data. Note: the channel path gain does not include the antenna gains. For a single record, the path gain may be computed from the calibrated and scaled channel response in the frequency domain as

$$
G=\frac{1}{g^{\mathrm{RX}} g^{\mathrm{TX}} N} \sum_{n=1}^{N} \frac{N}{\sum_{n=1}^{N_{f}}|\mathcal{F}[w(\tau)]|^{2}}\left|\frac{\mathcal{F}[w(\tau)] \times \mathcal{F}\left[S_{\text {meas }}(\tau)\right]}{\mathcal{F}\left[S_{\text {meas }}^{\mathrm{B} 2 \mathrm{~B}}(\tau)\right] / \mathcal{F}[A]}\right|^{2}
$$

The summation is over the frequency range of the record. The number of points in the frequency range equals the length of the $\mathrm{PN}$ sequence. In order to compute the average and standard deviation for an acquisition, we average the path gain over the number of records $N_{\text {rec }}$ within the acquisition using

$$
\begin{gathered}
G_{A v g}=\frac{1}{N_{r e c}} \sum_{p=1}^{N_{r e c}} G(p), \\
G_{S T D}=\frac{1}{\left(N_{r e c}-1\right)} \sum_{p=1}^{N_{r e c}}\left(G(p)-G_{A v g}\right)^{2} .
\end{gathered}
$$

We computed the standard deviation of the path gain using Eq. 2.27.

\subsection{Channel-Sounder Measurement Approach}

Parameters $S_{\text {meas }}, S_{\text {meas }}^{\text {B2B }}$, and $A$ of Eq. 2.19c we can derive from measurements taken using the channel sounder during an individual measurement run during a measurement campaign. A "measurement run" is a complete dynamic walk-through following a pre-established route or multiple static positions within a smart-manufacturing environment. A "measurement campaign" consists of the total number of measurement runs for a specific manufacturing environment. 
During the measurement campaigns, three smart-manufacturing environments were measured. These environments were the Central Utility Plant (CUP) at NIST Boulder, Colorado, an Automotive Assembly Plant (AAP), and the Machine Shop at NIST in Gaithersburg, Maryland. Measurements were also made at the Open Area Test Site (OATS) at NIST in Boulder, Colorado. Channel parameters were derived from measurements acquired with the process described here for all measurement campaigns.

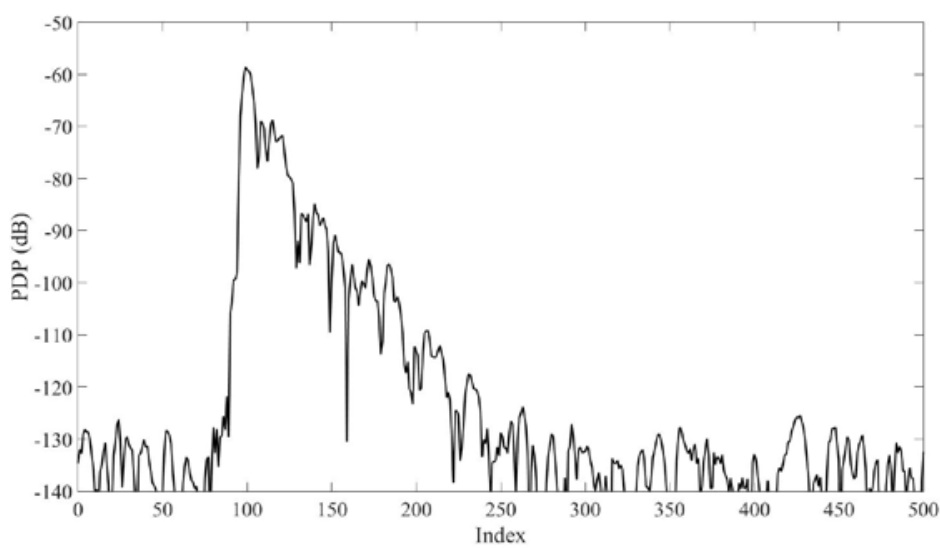

Fig. 2-6 PDP view on the Rx channel sounder.

Before each measurement campaign, a rigorous calibration of the channel-sounder hardware was conducted. This calibration and initial channel sounder set-up are discussed in detail in Section 3.1. The value of $50 \mathrm{~dB}$ was selected for $A$ for this calibration from Section 2.2 for all measurement campaigns and frequencies. After the initial set-up and before the measurement run, the channel sounder is used to measure the Back-to-Back signal $S_{\text {meas }}^{\text {B2B }}$ (see Eq. 2.19c) with the rubidium clock tied together. During this portion of the set-up, the PDP is processed in real time and its peak is inspected to ensure the drift is small and within acceptable limits. The PDP is displayed on the receiver's LabVIEW graph, as seen in Fig. 2-6. The PDP peak should stay within a few timing samples during the inspection. For example, if the PDP peak is positioned at sample 10, then it should only drift at most between samples 9 and 11 during the inspection. Any more drift would indicate poor synchronization between the rubidium clock or timing drift within the timing distribution circuitry in the TX and RX chassis. To ensure proper synchronization between the rubidium clock, the clocks are disciplined together for 24 hours before the measurement campaign, before the Backto-Back signal measurement.

\subsubsection{Channel-Sounder Measurement Approach for Mobile Measurements}

During the smart-manufacturing mobile measurement campaigns, the TX and RX were loaded onto individual carts with soft wheels and plastic tops. The soft wheels helped reduce the bounce when pushing the channel sounder through the manufacturing environment. The RX was mobile while the TX was stationary during all measurement campaigns.

Before a measurement run may be conducted, a route through the manufacturing environment is typically mapped out with check points (CPs). These check points can be seen in Fig. 2-7 as the numbers in the green boxes. The check points are marked on the floor with duct tape on the floor and measuring the distance between the duct tape location (in $\mathrm{x}$ and $\mathrm{y}$ coordinates) to a global origin. While the global origin must be held constant through an entire measurement campaign, the route through the manufacturing environment may changed as long as the check points are annotated. Ambiguity in the $\mathrm{x}$ and $\mathrm{y}$ coordinates of the check points from true $\mathrm{x}$ and $\mathrm{y}$ coordinates 
contributes to uncertainty in range when plotting against range or a $2 \mathrm{D}$ representation of the manufacturing environment, as discussed in Appendix D.

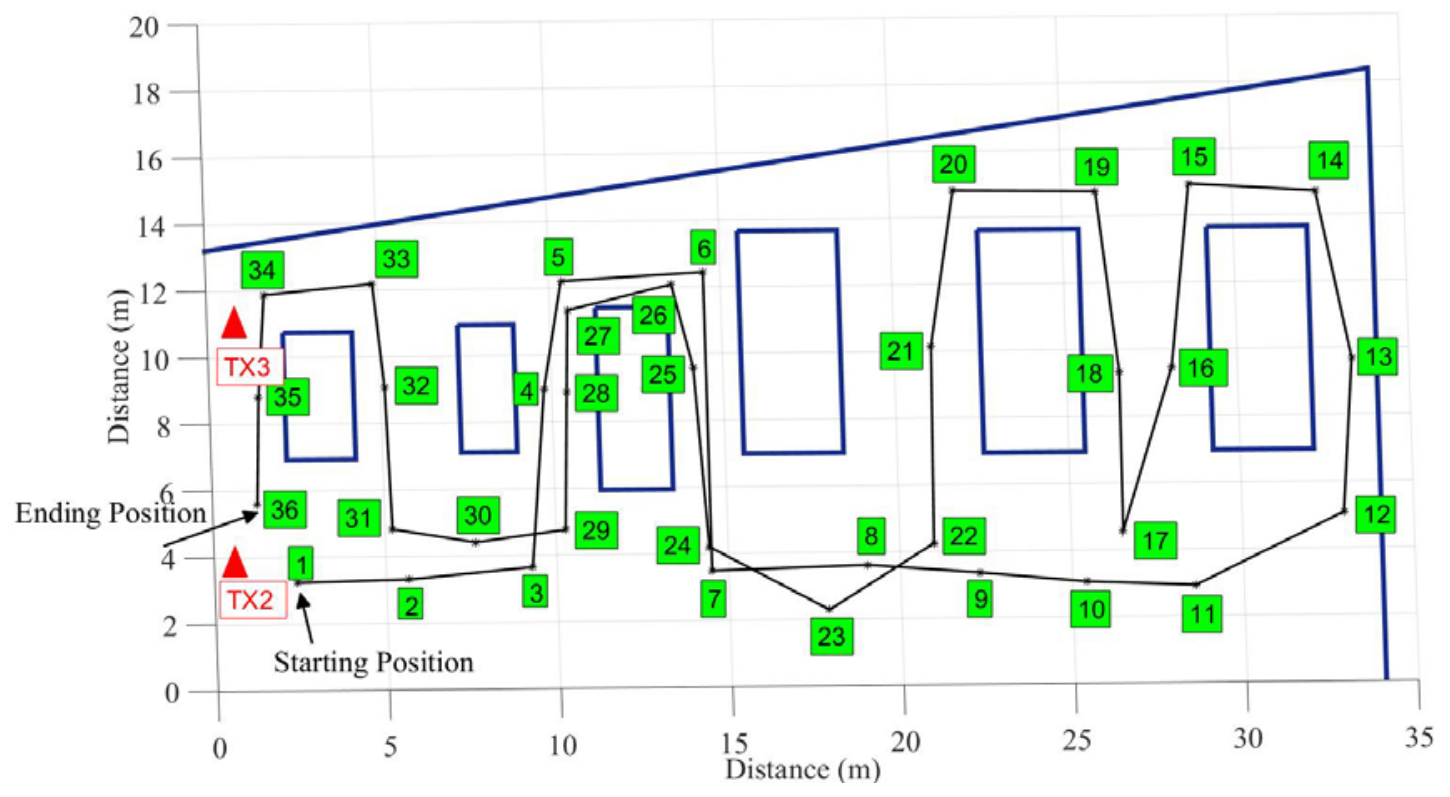

Fig. 2-7 Central Utility Plant (CUP) measurement route.

A measurement run requires two to three people to perform the channel-sounder measurements through the manufacturing environment. One person pushes the cart, containing the RX. Another person calls out the check point number when the cart's left or right front wheel moves over the check point duct tape marker. The third person, if available, writes down the acquisition number on the test sheet reported by the RX software at the moment the check point is called out. An example of a test sheet is shown in Fig. 2-8. This measurement process ensures a tight linkage between the acquisition number and the check point. Variability in this linkage can be considered as uncertainty. A discussion of position variability can be found in Appendix D.

At the start of a measurement run, the RX is placed at a check point, usually check point 1 , and the acquisition of data at the RX is started. The cart is pushed at a walking pace along the route. At the end of the route, the data acquisition is stopped. Typically, a second $S_{\text {meas }}^{\text {B2B }}$ measurement is performed for the same

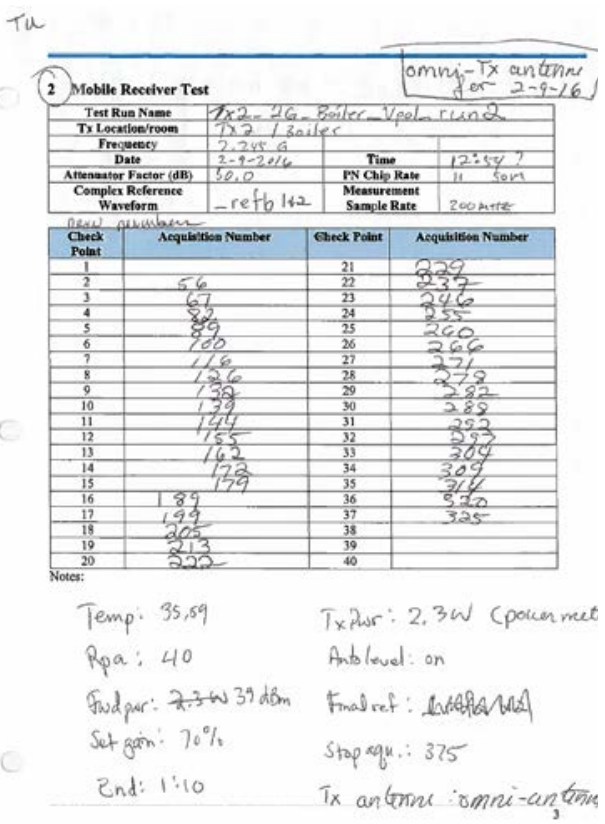

Fig. 2-8 Example test sheet for acquisition number and check point linking. attenuation as the first $S_{\text {meas }}^{\mathrm{B} 2 \mathrm{~B}}$ measurement to study the effects of environmental temperature drift and of the impact of moving the RX cart. Once again, during this measurement of the $S_{\text {meas }}^{\mathrm{B} 2 \mathrm{~B}}$, the 
graph of the PDP on the RX is viewed to inspect whether any drift of the PDP peak that had occurred during the measurement run.

\subsubsection{Channel Sounder Measurement Approach for Fixed-Position Measurements}

Both the TX and RX carts were held stationary during the manufacturing fixed-position measurement campaigns. The RX cart was placed at a check point. The RX antenna was mounted with the antenna positioner seen in Fig. 2-9(a). The initial position of the antenna was located at the edge of the RX cart. This starting position was chosen to minimize the risk of catching a cable on pipes or other obstructions that may have possibly been around the cart. The parameter $S_{\text {meas }}$ was measured when the antenna positioner was stationary, then the antenna positioner moved slowly to the new position and $S_{\text {meas }}$ was measured once again. The antenna positioner moved from the edge of the cart to various locations over the surface of the cart. The resulting antenna positions are shown in Fig. 2-9(b). The Antenna Mount Positions in Fig. 2-9(b) are referenced to the TX antenna position. As with the mobile measurements, a back-to-back measurement for $S_{\text {meas }}^{\mathrm{B} 2 \mathrm{~B}}$ was conducted before and after the measurements.

\subsection{Channel Sounder Data Acquisition}

Prior to a measurement campaign, the user inputs the desired $\mathrm{PN}_{\text {ideal }}$ length, Symbol Rate, and

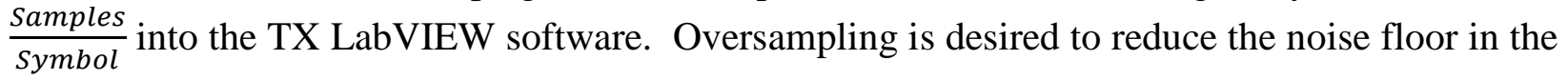
measurements. The user inputs the number of records to be collected within an acquisition, the time delay in units of the number of codewords transmitted between each of those records, the time delay in milliseconds between each acquisition, and the number of acquisitions to be collected before closing the file and opening a new measurement file during a measurement run. The TX input parameters are chosen with knowledge of the estimated cart speed and other experimental parameters to ensure data will capture fast fading as well as path-gain statistics and other slow-
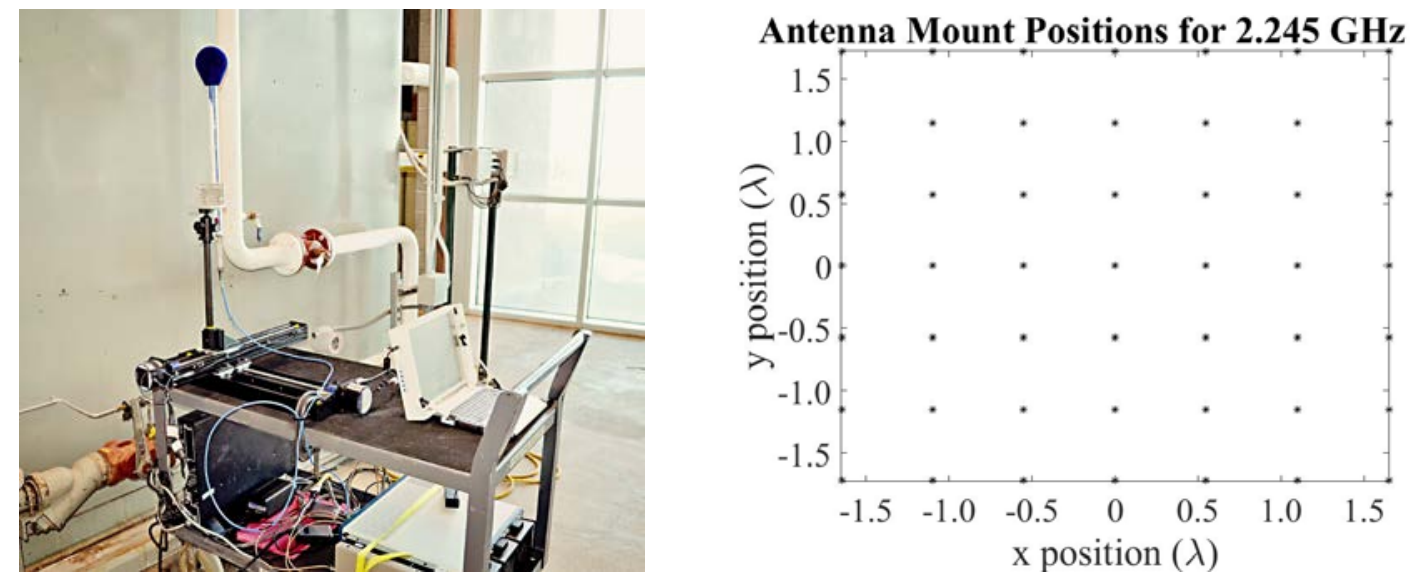

Fig. 2-9 (a) RX antenna mounted on the antenna positioner for fixed measurements and (b) antenna mount positions for check point 8 and a transmitter position of TX2.

fading behavior. The number of acquisitions in a file is chosen to avoid significantly slowing down the RX controller when saving the measurement data to the RAID hard drive. 


\subsection{Channel Sounder Post-Processing}

Path gain is computed from a measurement run by use of the channel sounder theory. The post processing of the channel sounder data takes a few steps. The first step us to convert the NI tdms data format into a more flexible post-processing and storage format such as MATLAB mat. An open-source software program performed the conversion from tdms to mat formats. A NIST software program was written to post process the measurement data such as applying the back-toback, filtering, and calculation of the PDP.

Next, $S_{\text {meas }}^{\mathrm{B} 2 \mathrm{~B}}$ measurements are checked to ensure that the data are usable. This step is vital for suitable post-processing. Fig. 2-10(a) shows a usable PDP result with $-80 \mathrm{~dB}$ noise floor and an impulse response at $0 \mu \mathrm{s}$. If the $S_{\text {meas }}^{\mathrm{B} 2 \mathrm{~B}}$ measurement provides poor performance such as the $-20 \mathrm{~dB}$ noise floor seen in Fig. 2-10(b), it should not be used in the final data analysis. Another parameter to be aware of is the overload flag; if the reference file has the overload flag set to 1 then an overload event occurred during the measurement. An overload event occurs when the RF signal starts to exceed the expected input power of the RX channel sounder. If this occurs, this will result

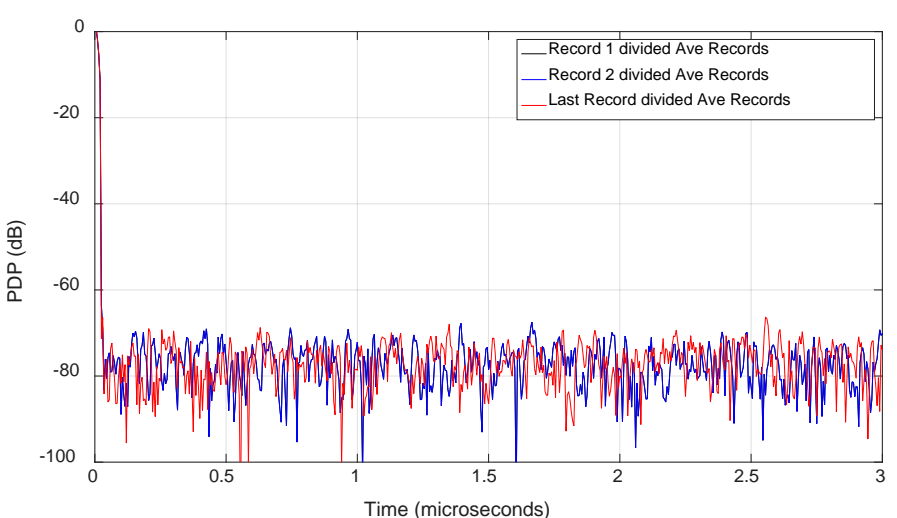

(a)

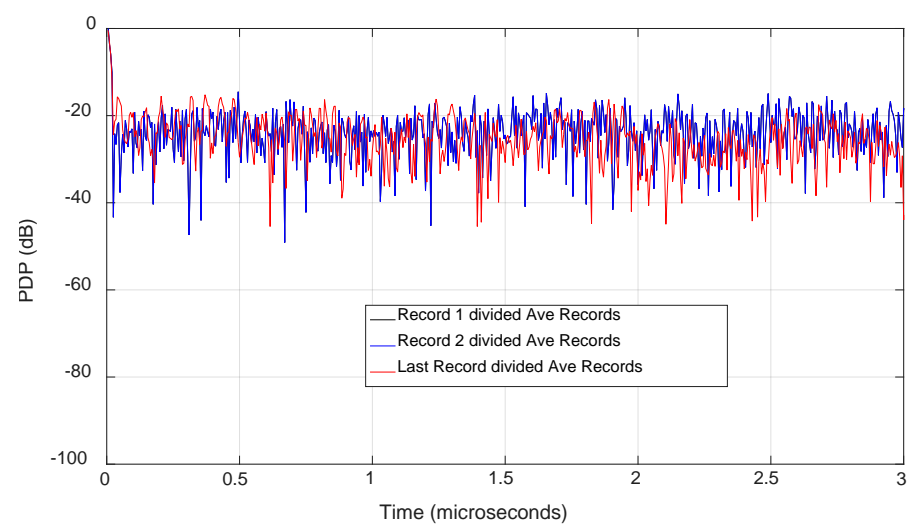

(b)

Fig. 2-10 (a) Usable reference measurement for channel sounder post-processing, (b) unusable reference measurement.

format are converted to a numerical array of $S_{\text {meas }}$ values. The size of the numerical array is a 2D in a poor reference measurement. Other poor references may occur due to loose connections in the cables, broken cables or attenuators, or operator error. Fig. 2-10(a) and (b), showing usable and unusable references, were measured with a $50 \mathrm{~dB}$ attenuation at $30 \mathrm{dBm}$ output power.

Another challenge with the reference file occurs when choosing the attenuation value for the B2B. If the output power from the TX is low $(\sim 0 \mathrm{dBm}$ for our system), a lower value of attenuation such as $20 \mathrm{~dB}$ will provide a more favorable reference file compared to an attenuation of $50 \mathrm{~dB}$. If the output power is higher (such as $30 \mathrm{dBm}$ for our system), then a higher value of attenuation of $50 \mathrm{~dB}$ will be suitable to protect the $\mathrm{RX}$ from possible damage. Fig. 2-11 shows typical reference files produced by $20 \mathrm{~dB}$ attenuation and $50 \mathrm{~dB}$ attention, respectively.

After inspection of the $S_{\text {meas }}^{\mathrm{B} \text {, }}$, all other measurement records in the NI tdms
The size of the numerical array is a 2D 
array. The first dimension equals the $\mathrm{PN}_{\text {ideal }}$ sequence length times the number of samples/symbol times. The second dimension equals the total number of records for the measurement run. The $\mathrm{PN}_{\text {ideal }}$ sequence length times the samples/symbol equals the $\mathrm{PN}_{\text {ideal }}$ sequence length. Each record in this numerical array is post-processed with $S_{\text {meas }}^{\mathrm{B} \text { 2B }}$, as described in Section 2.1.1.

At this point in the post processing, the channel impulse response $y(t)$ has been computed and stored into a numerical array. In our processing, $y(t)$ consist of in-phase and quadrature components as opposed to a real-valued time-domain waveform. This numerical array is saved to a .mat file format with additional metadata in the .mat file. The metadata is contained in the excel file: SmartManufacturing_MetaData. This spreadsheet and .mat files are contained on a NIST server and can be provided to the public through a web portal, http://doi.org/10.18434/T44S3N [10].

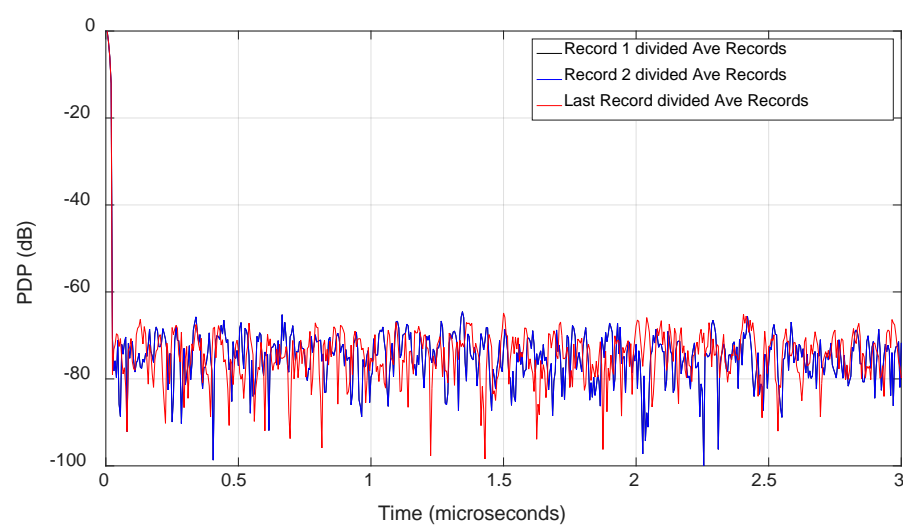

(a)

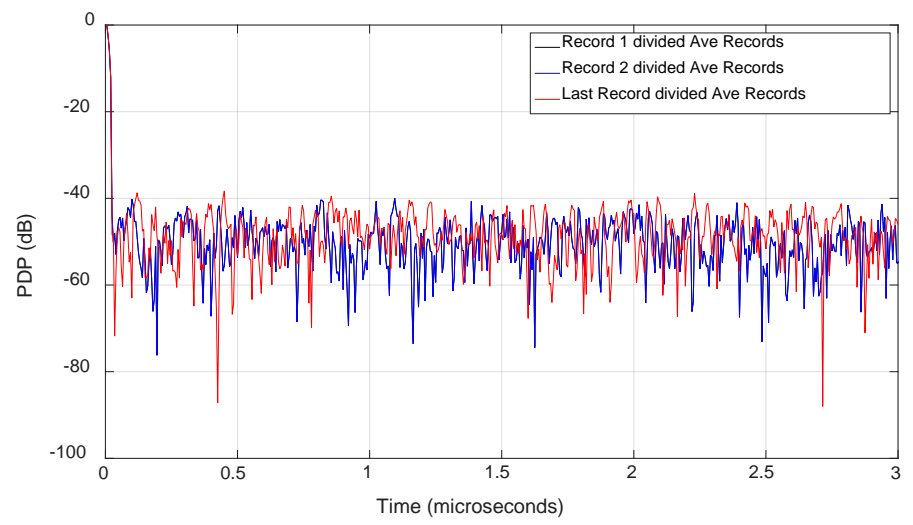

(b)

Fig. 2-11 (a) Reference measurement for $20 \mathrm{~dB}$ attenuation with $0 \mathrm{dBm}$ output power. (b) Reference measurement for 50 $\mathrm{dB}$ attenuation with $0 \mathrm{dBm}$ output power.

\subsubsection{Correlation-Based Measurements Two-Slope Polynomial Fit}

A log-distance path gain model [11] was applied to the path gain measurements. The model is

$$
\begin{array}{cc}
Y(r)=B 1 \times 10 \log _{10}(r)+A 1 & r \leq r_{B P} \\
Y(r)=B 2 \times 10 \log _{10}(r)+\left(A 2+r_{B P}(B 2-B 1)\right) & r>r_{B P}
\end{array}
$$

where $\mathrm{Y}$ is the resulting log-distance path gain model. B1 and B2 are the path gain exponent and is dimensionless. A1 is the path gain with respect to the minimum range of 5 or 10 meters depending upon the measurement campaign. A2 is the path gain at $r_{B P}$, where $\mathrm{r}$ is the distance between the transmitter and the receiver in linear scale. The break point $r_{B P}$ was kept constant between similar dimension campaign routes. This allows for a comparison between the measurement campaigns. 


\section{Channel-Sounder Configuration and Validation}

A block diagram of the channel-sounder system is shown in Fig. 3-1. There are multiple tests that must be accomplished before the channel sounder data may be processed to provide wirelesschannel metrics. A description and information about the NIST set-up and verification tests are given next. A list of the NI components is provided in Appendix A.

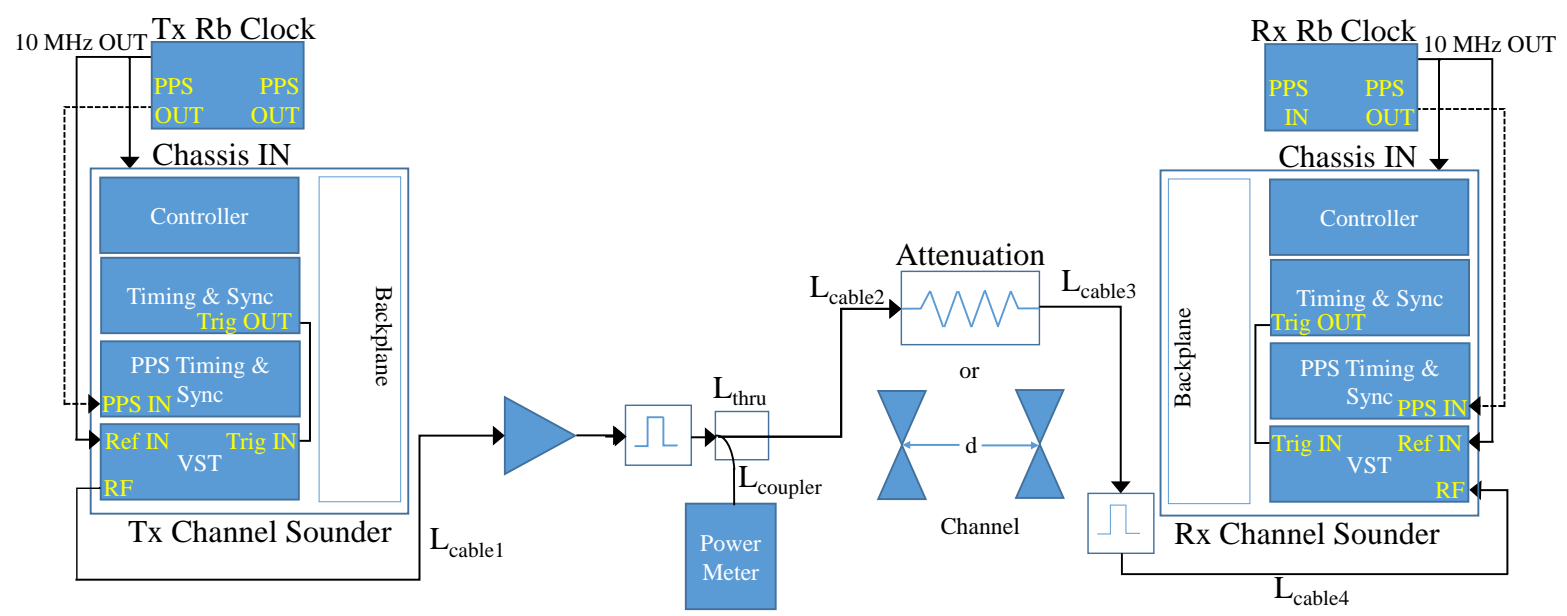

Fig. 3-1 Block diagram of the channel sounder architecture.

\subsection{Initial Set-up and Channel Sounder Calibration}

The initial set-up and calibration of the channel sounder requires a series of measurements to check drift, linearity, and to correct for system losses. These measurements are described below.

\subsubsection{Stabilizing the rubidium clock}

This step must be done approximately 72 hours before all measurement campaigns. The set-up for this step is seen in Fig. 3-2. The TX rubidium clock PPS OUT is connected to the PPS Timing \& Sync module in the TX. The RX rubidium clock PPS OUT is connected to the PPS Timing \& Sync module on the RX. The PPS OUT of the RX rubidium clock is connected to the PPS IN of the TX rubidium clock. If the PPS outputs are not being used, terminate with a capacitor end cap. Note: The Fan Setting Must Be On High.

Next, the LabVIEW codes that set the time reference and discipline the clocks are run on both the RX and TX systems. See Tables 3-1 and 3-2, respectively, for input values. To ensure the accuracy specified in Table 8-3, this procedure must be conducted at least 72 hours before the measurement.

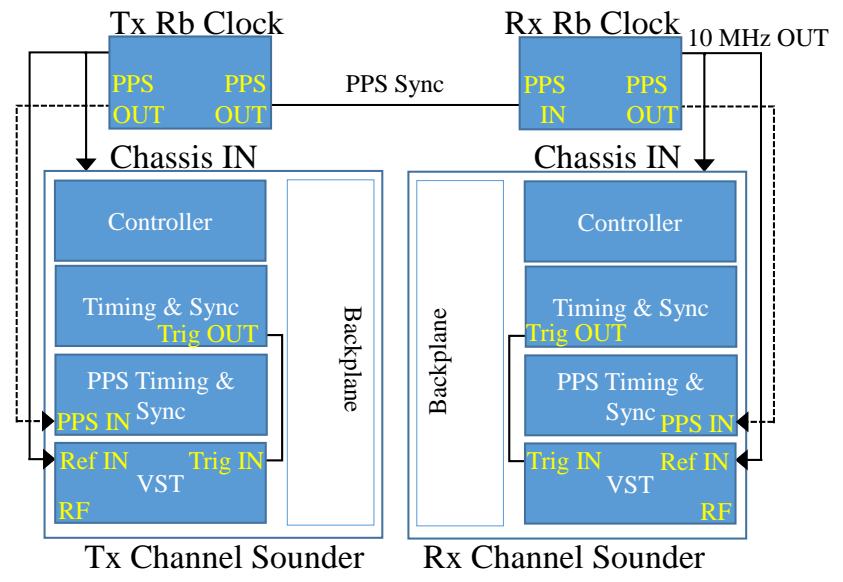

Fig. 3-2 Stabilizing rubidium clocks. 


\subsubsection{Rubidium Clock Drift Measurement}

This measurement determines the drift between the TX rubidium clock and the RX rubidium clock. The set-up for this measurement can be seen in Fig. 3-3. This setup is very similar to the Stabilizing of the rubidium clock set-up in Fig. 3-2. The main difference is the use of an oscilloscope to measure the drift between the rubidium clock. Using a splitter, we connect the $10 \mathrm{MHz}$ TX rubidium clock to the TX and an oscilloscope. Using another splitter, we connect the $10 \mathrm{MHz}$ $\mathrm{RX}$ rubidium clock to the $\mathrm{RX}$ and the oscilloscope. Initially the PPS OUT from the $\mathrm{RX}$ rubidium clock should be connected to the PPS IN of the TX rubidium clock after the clocks warm up for 72 hours in order to obtain

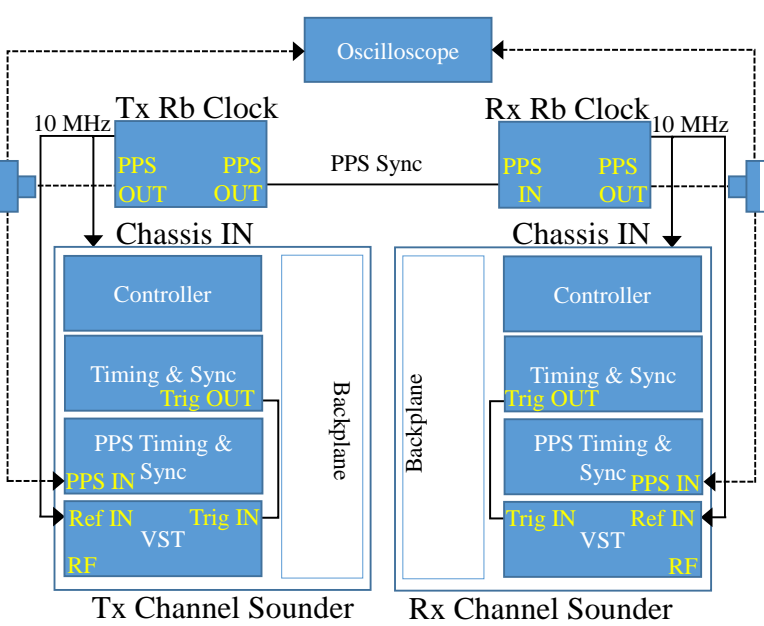

Fig. 3-3 Rubidium clocks drift measurement set-up. absolute timing. The 72 hours requirement comes from an empirical observation of the resolution and stability for measurements conducted on a moving cart in an out-of-laboratory environment. If the measurements with the channel sounders are conducted in a controlled laboratory setting,

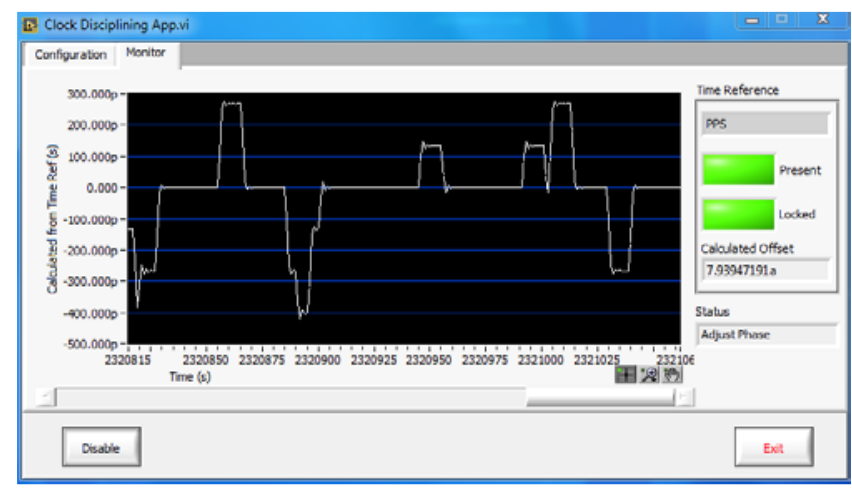

(a)

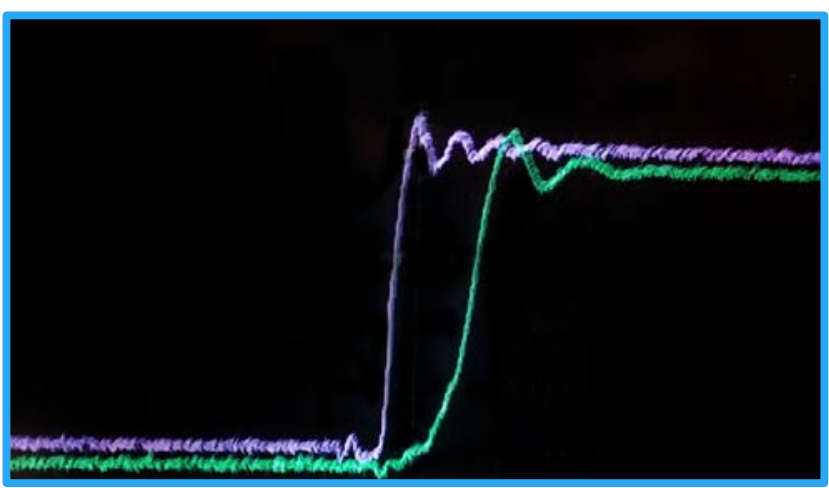

(b)

Fig 3-4 (a) Clock discipline LabVIEW program with poor synchronization and (b) rubidium clock leading edge measurement. then 24 hours would generally be sufficient.

After the 72 hour warm-up time, the oscilloscope is turned on. The oscilloscope is set to measure the difference between the leading edge of the $10 \mathrm{MHz}$ TX and RX rubidium Clock reference signals. The LabVIEW program that disciplines the clock is run on the RX system. This software plots the "Calculated from Time Ref" vs time. This plot provides the measured stability of the channel-sounder timing circuits. The “Calculated from Time Ref” plot should have values in the picoseconds and not nanoseconds, as seen in Fig 3-4 (a). If Fig 3-4 (a) is shown in the LabVIEW program during this measurement, then there is drift that must be corrected before measurements can be conducted. If the "Calculated from Time Ref" plot has values in picoseconds, then disconnect the PPS IN on the TX rubidium clock from the PPS OUT of the RX clock. With the PPSs now disconnected and the rubidium clock 
still connected to the oscilloscope, the leading edges of the rubidium clock should still be held constant with only picoseconds of drift between the clocks, as seen as in Fig 3-4(b). If this is found to be true, then next steps of the measurement set-up for the channel sounders can be conducted. Future work will investigate the long term stability and timing of the channel sounder for indoor and outdoor use. The specification and parameters of the rubidium clock may be found in Appendix C.

\subsubsection{Power Amplifier Linearity Measurement}

This measurement finds the linear range of the power amplifier when combined with the TX of the channel sounder. If the clocks are turned off after the rubidium clock drift measurement, then the set-up must be warmed up again to ensure proper distribution of the clock information through the TX and RX.

To ensure protection of the RX, an attenuator is connected between the power amplifier and the RX VST. The attenuation for the measurement campaign is chosen by reviewing the PDPs for saturation and noise floor. The attenuation values are chosen to provide the best dynamic range, without clipping or falling below the noise floor of the RX hardware during the measurement campaigns. For the measurements reported here, $50 \mathrm{~dB}$ was used.

With $50 \mathrm{~dB}$ attenuation set between the TX and RX of the channel sounder, a single acquisition is measured with the RX. Initially, a very-low power setting for the power amplifier was used by controlling the TX VST's output power level. For the system shown here, the power level starting value was $-30 \mathrm{dBm}$, then a sequence was measured. This process continued in $1 \mathrm{~dB}$ increments to -6 dBm, see Table 3-2.

\subsubsection{Power Budget Measurement}

This measurement determines the power budget for the channel sounder for an attenuation of 50 $\mathrm{dB}$. The losses for multiple components may be measured with a spectrum or signal analyzer. After the measurement of the components, a power budget analysis is performed for the set-up seen in Fig. 3-5. The value of the attenuation is chosen to protect the Power Meter from excessive output power from the power amplifier.

Table 3-1 OATS measurement campaign component losses.

\begin{tabular}{|l|c|c|}
\hline \multicolumn{1}{|c|}{ Component } & Losses (dB) at 2.245 GHz & Losses (dB) at 5.4 GHz \\
\hline Attenuation & 50.12 & 50.23 \\
\hline Cable 3 & 0.5 & 0.5 \\
\hline Coupler Thru Line & 0.46 & 0.51 \\
\hline Coupler Coupled Line & 21.5 & 20.515 \\
\hline Cable 2 & 2.03 & 2.4 \\
\hline Power Meter Offset Value & $19.01 \leftarrow(21.5-0.46-2.03)$ & $17.61 \leftarrow(20.515-0.51-2.4)$ \\
\hline
\end{tabular}

For the set-up shown here, a Keysight PXA signal analyzer was used to measure the loss in Cable 2, Coupler, Attenuation and Cable 3 by comparing the power at the output of Cable 3 to the power at the coupler. Note that these components could also be measured with a vector network analyzer. Table 3-1 shows typical losses for these components for the measurement campaign. 
The power meter offset value shifts the power meter reference plane to the star location. With the known component losses, the power budget is computed at the input to the attenuator as shown by the star in Fig. 3-5. The power meter power reading was compared to the PXA power reading for different TX settings, as seen in Table 3-2. The OATS measurement campaign power budget in Table 3-2, with power meter offsets from Table 3-1, is used to determine the output power for the power amplifier. Similar power budgets were measured for the other measurement campaigns.

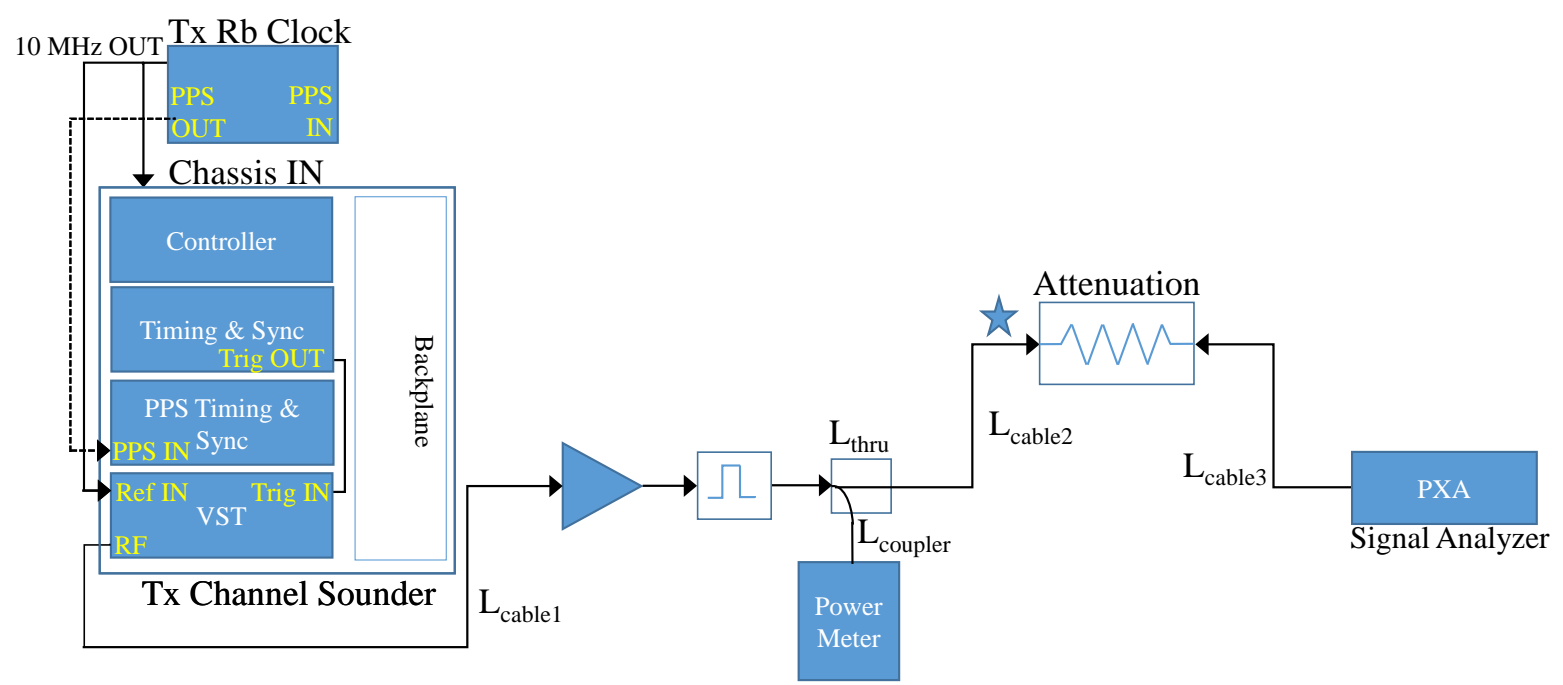

Fig. 3-5: Power budget measurement set-up.

Table 3-2 OATS power budget.

\begin{tabular}{|c|c|c|}
\hline TX Setting (dBm) & $\begin{array}{c}\text { Power Meter Measured Value (dBm) } \\
\text { at Star Location for } \mathbf{2 . 2 4 5} \mathbf{~ G H z}\end{array}$ & $\begin{array}{c}\text { PXA Measured Value (dBm) at } \\
\text { Star Location for 2.245 GHz }\end{array}$ \\
\hline-30 & 13.43 & 12.62 \\
\hline-20 & 23.50 & 22.72 \\
\hline-16 & 27.57 & 26.72 \\
\hline-14 & 29.50 & 28.82 \\
\hline-12 & 31.54 & 30.82 \\
\hline-10 & 33.54 & 32.72 \\
\hline-9.4 & 34.24 & 33.42 \\
\hline-9 & 34.62 & 33.82 \\
\hline-8 & 35.56 & 34.82 \\
\hline-6 & 37.63 & 36.82 \\
\hline TX Setting (dBm) & Power Meter Measured Value (dBm) & PXA Measured Value (dBm) at \\
\hline-30 & at Star Location for 5.4 GHz & Star Location for 5.4 GHz \\
\hline-25 & 13.43 & 13.00 \\
\hline-20 & 18.33 & 18.03 \\
\hline-15 & 23.30 & 22.93 \\
\hline-10 & 27.61 & 27.83 \\
\hline-9 & 32.96 & 32.53 \\
\hline-8 & 33.86 & 33.43 \\
\hline-7 & 34.75 & 34.23 \\
\hline-6 & 35.59 & 35.11 \\
\hline-5 & 36.41 & 35.93 \\
\hline & 37.19 & 36.63 \\
\hline
\end{tabular}




\subsection{Open Area Test Site (OATS) Measurement Campaign}

To validate the channel-sounder measurements and processing, we compared the path loss vs. range between the channel-sounder measurements and simulated results. The OATS is a measurement location at NIST, Boulder, seen in Fig. 3-6. It is located in an open field with an electrically conducting mesh over an area of 40 meters by 60 meters.

We measured the $S_{\text {meas }}^{\mathrm{B} 2 \mathrm{~B}}$ was measured using $50 \mathrm{~dB}$ attenuation between $\mathrm{TX}$ and RX. Next, the RX antenna was

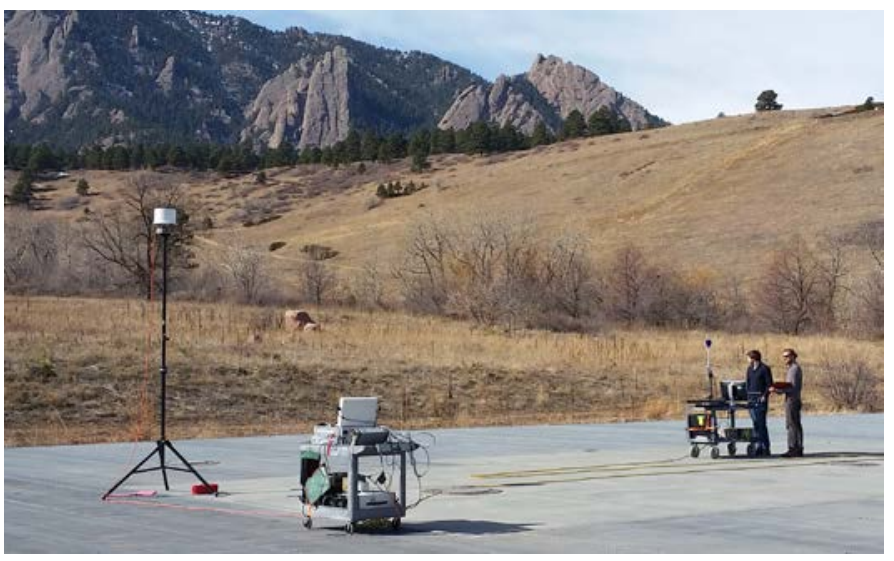

Fig. 3-6 Channel sounder deployment at NIST OATS facility. positioned 1.5 meters away from the TX antenna. At this position, $S_{\text {meas }}$ was measured for 10 acquisitions with 40 records/acquisition. The RX cart was stationary. The RX cart was then moved in 1 meter increments to a 20 meter separation from the TX antenna and then in 2 meter increments to a 40 meter separation from the TX antenna. At each position, 10 acquisitions of $S_{\text {meas }}$ were measured while the RX cart was stationary. We conducted two measurement runs using this approach. The measurement runs used the ETS-Lindgren directional double-ridged guide TX antenna for operating frequencies of 2.245 $\mathrm{GHz}$ and $5.4 \mathrm{GHz}$, also described in Section 2.1. The RX antenna was the Seibersdorf Laboratories omnidirectional dipole.

In post processing, we computed the PDP for each $\mathrm{RX}$ position using a $\mathrm{PN}_{\text {ideal }}$ window with discrete sum scaling for $w(\tau)$ from (2.25). The path gain was computed from the PDP by subtracting off the gains of the TX and RX antennas, see (2.29).

We computed the measured path gain from the OATS is compared to a Uniform Theory of Diffraction (UTD) ray-tracing simulation using the Numerical Electrical Computer-Basic

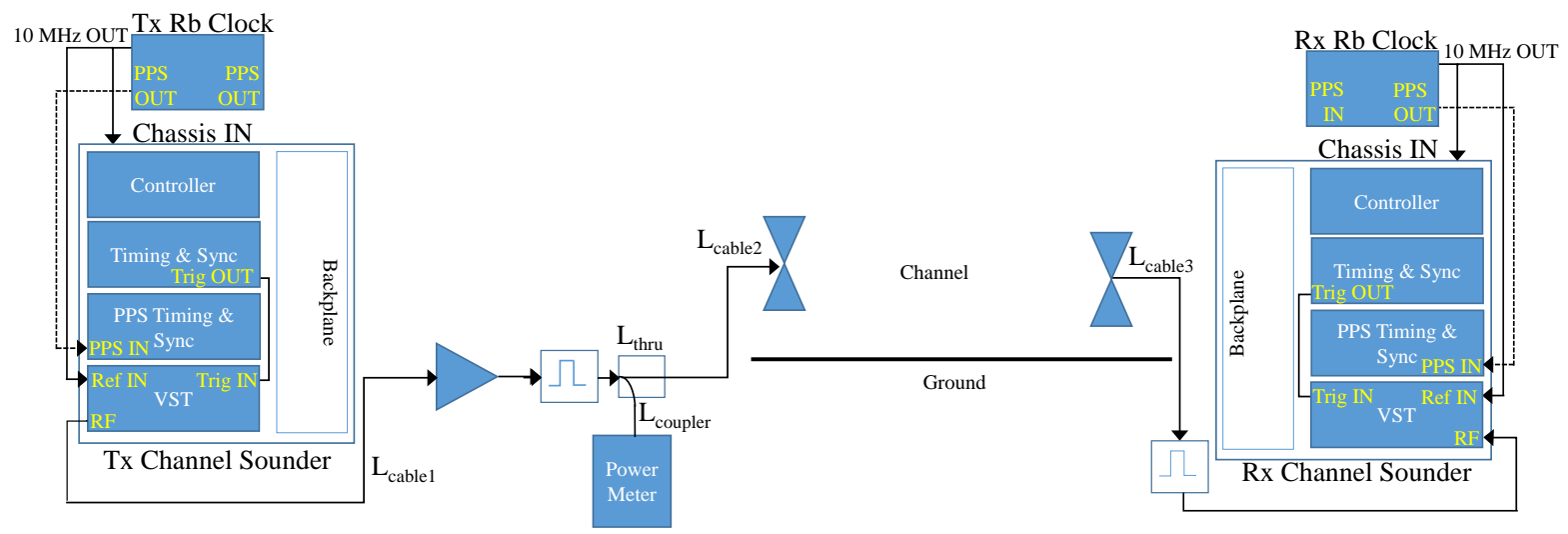

Fig. 3-7: Measurement of path gain set-up for the OATS. 
Scattering Code [12]. The simulation accounts for a ground bounce from a perfect electrically conducting (PEC) ground. The antenna effects were simulated by use of a horn model. The geometrical set-up for the simulation is shown in Fig. 3-7. Finally, the path gains for $2.245 \mathrm{GHz}$ and $5.4 \mathrm{GHz}$ operating frequencies are shown in Fig. 3-8. For simplicity, we used a polynomial fit for the path gain that lead to a slope of $2.1 \mathrm{~dB}$ for $2.245 \mathrm{GHz}$ and $2.0 \mathrm{~dB}$ for $5.4 \mathrm{GHz}$, where 2.0 would match with free space. Fig. 3-8 shows the path gain comparison between the polynomial fit of the OATS measurement data to the ray-tracing simulation. The ray-tracing simulation slope for $2.245 \mathrm{GHz}$ operating frequency is $2.3 \mathrm{~dB}$ and $2.1 \mathrm{~dB}$ for $5.4 \mathrm{GHz}$. The ray tracing results are also provided in green in the following figures. The blue plot is the linear regression of the channel-sounder measurements. The black dashed line plot is the simulated freespace result. 


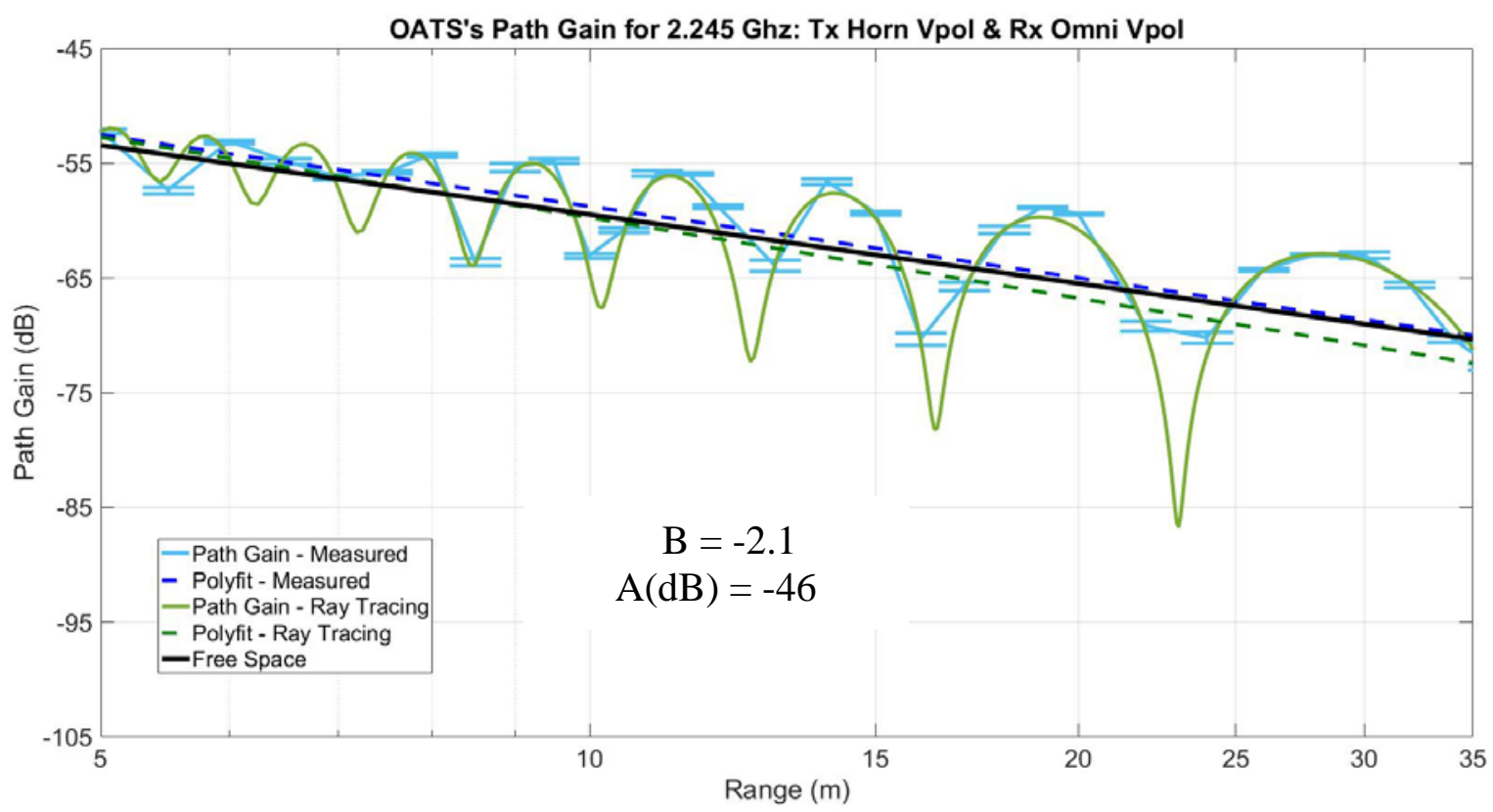

(a)

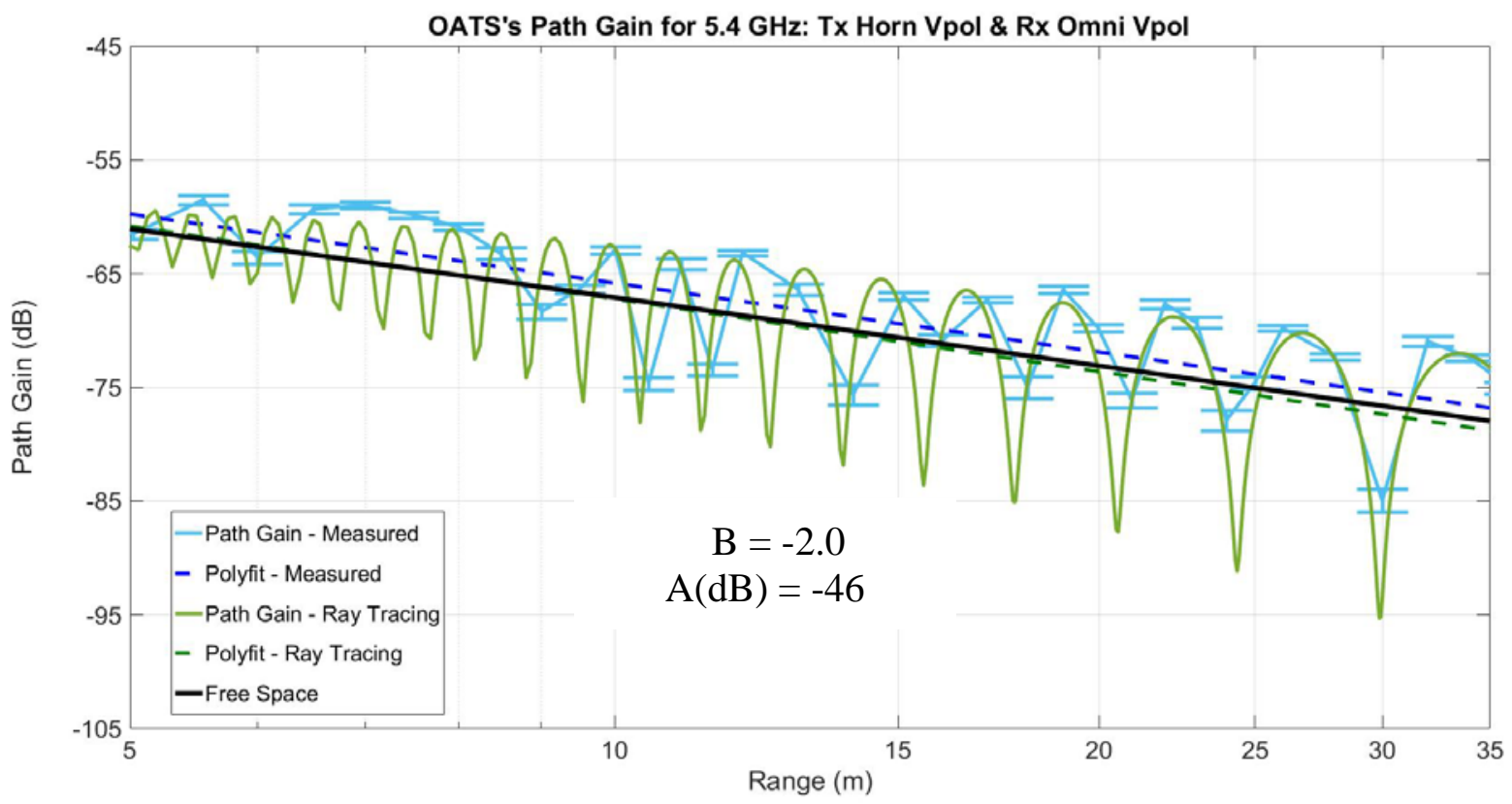

(b)

Fig. 3-8 Path gain comparison to ray tracing at the OATS for (a) $2.245 \mathrm{GHz}$ and (b) $5.4 \mathrm{GHz}$. 


\section{Channel Measurements in Representative Facilities}

\subsection{NIST Central Utility Plant (CUP) Measurement Campaign}

The Central Utility Plant (CUP) on the NIST Boulder campus is an indoor industrial steam generation plant containing large machinery such as boilers with multiple overhead obstructions, as seen in Fig. 4-1. The outer floor dimensions are approximately $50 \mathrm{~m}$ x $80 \mathrm{~m}$ with a ceiling height of approximately $7.6 \mathrm{~m}$. The floor was separated into one section for refrigeration equipment and with boilers in the other section. The measurement campaign stayed in the boiler section due to construction in the refrigeration section during the time of the measurement campaign. The sections were separated by a large cinder block wall with two openings of $3 \mathrm{~m}$, as seen in Fig. 4-2. This figure contains the floor plan of the boiler section with the mobile measurement check point paths. The boiler section was approximately $20 \mathrm{~m}$ x $80 \mathrm{~m}$.

During the measurement campaign, the transmitter was placed at two locations. Table 4-1 contains the TX position information with the origin reference (orange circle) shown in Fig. 4-2. The transmit antenna was placed at one of two locations during the measurement campaign. The first position, shown in Fig. 4-2, was at the top left corner on the boiler floor of the CUP facility. This is denoted TX2 because it was on the second floor above the basement. TX2 was located on the same floor as the RX antenna. The second location for the transmit antenna was on the upper floor of the CUP facility. This floor had an open view of the first floor with a single safety rail. This is denoted as TX3. The receive antenna was the omnidirectional dipole described in Section 2.1.

Table 4-1: CUP industrial steam plant TX locations.

\begin{tabular}{|c|c|c|c|}
\hline $\begin{array}{c}\text { TX } \\
\text { Location }\end{array}$ & $\mathbf{X ( m )}$ & $\mathbf{Y ( m )}$ & $\mathbf{Z ( m )}$ \\
\hline $\mathrm{TX} 2$ & 1.14 & 1.95 & 3.36 \\
\hline $\mathrm{TX} 3$ & -1.45 & 10.64 & 6.22 \\
\hline
\end{tabular}

We conducted the channel-sounder measurements for this campaign during the spring of 2016 using the previouslydescribed channel-sounder system with a symbol rate of 50 Msym/s, $4 \frac{\text { Samples }}{\text { symbol }}$ and a PN code of 2047 symbols

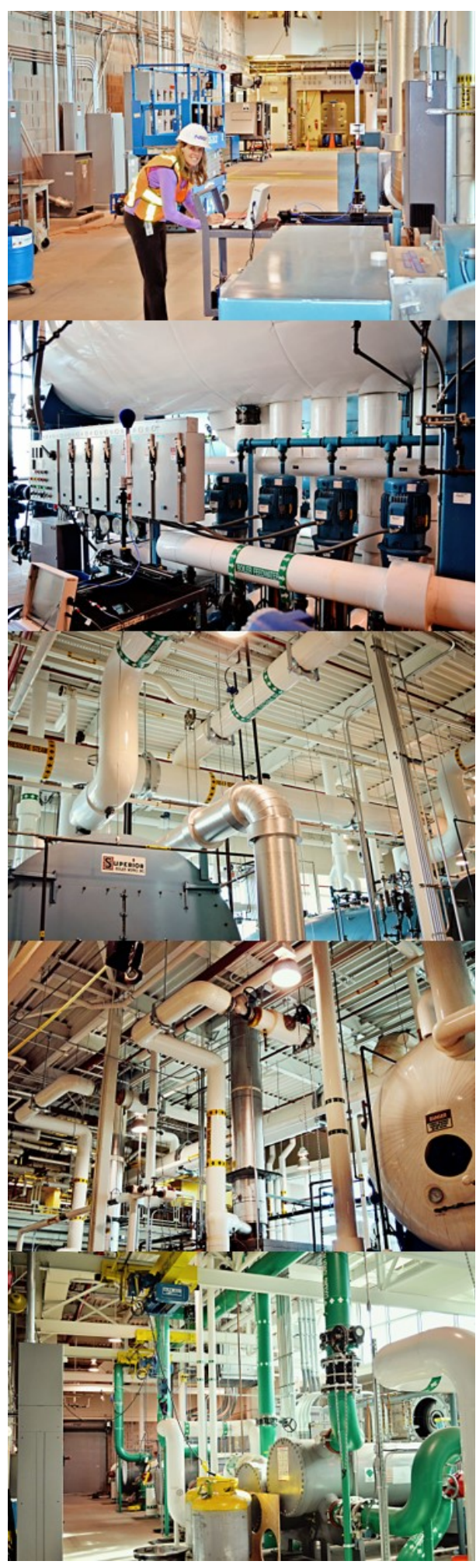

Fig. 4-1 CUP industrial steam plant. 
corresponding to a PN maximum length sequence of 11 . The attenuation for the back-to-back measurement was $50 \mathrm{~dB}$. The two types of transmit antennas described in Section 2.1 were used. The post processing of the measurement data used a PNideal filter with discrete sum scaling.

The data collected during the measurement campaigns can be accessed at the NIST website http://doi.org/10.18434/T44S3N.

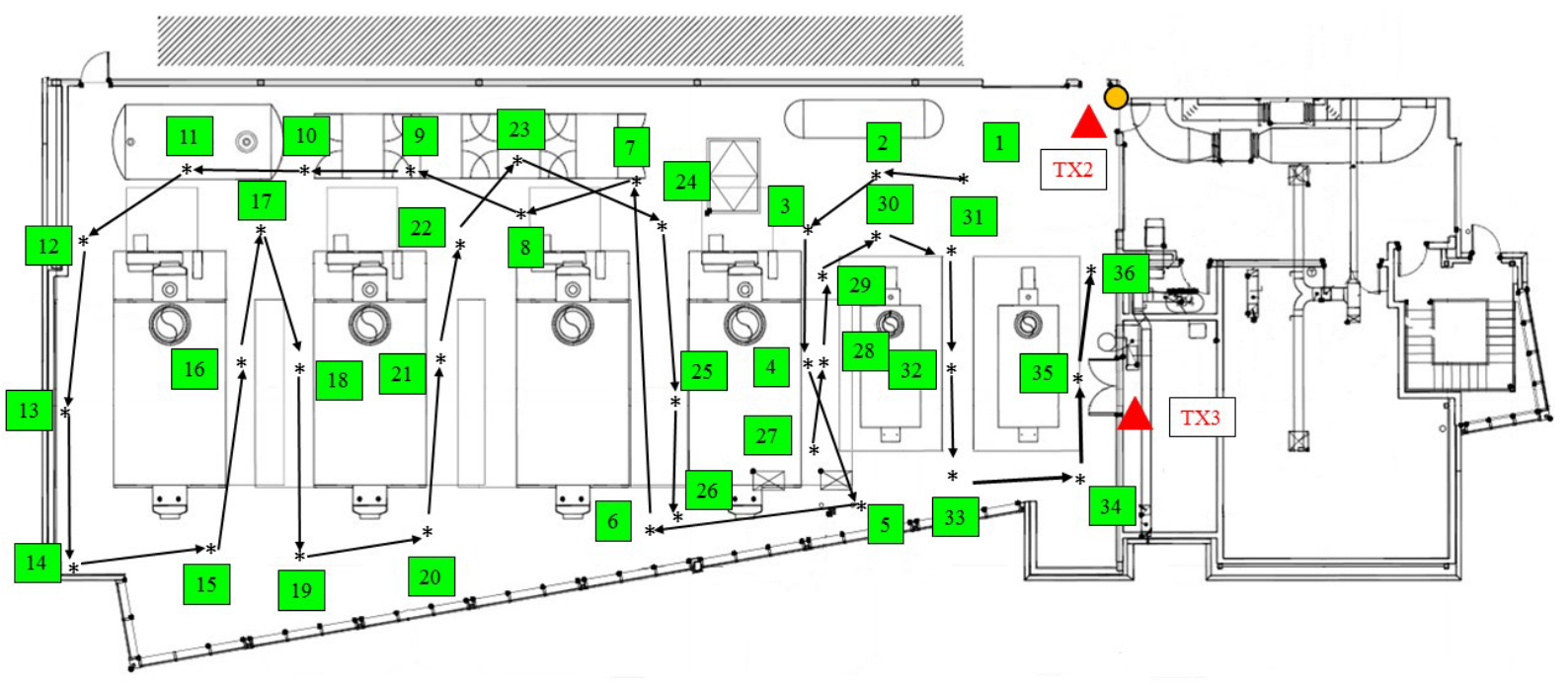


Table 4-2: CUP mobile runs: omnidirectional = biconical antenna and horn = double-ridged guide antenna .

\begin{tabular}{|c|c|c|c|c|c|c|}
\hline $\begin{array}{c}\text { Channel } \\
\text { Sounder } \\
\text { Information }\end{array}$ & $\begin{array}{l}\text { Frequency } \\
\text { (GHz) }\end{array}$ & $\begin{array}{c}\text { TX } \\
\text { Position }\end{array}$ & $\begin{array}{c}\text { Path Gain Model } \\
\text { B, A(dB) }\end{array}$ & $\begin{array}{l}\text { Acquisition } \\
\text { Numbers } \\
\text { (start,end) }\end{array}$ & $\begin{array}{c}\text { Figure } \\
\#\end{array}$ & $\begin{array}{c}\text { Measurement } \\
\text { Filename }\end{array}$ \\
\hline $\begin{array}{l}\text { RX: Vertical Pol. } \\
\text { TX: Vertical Pol. } \\
\text { Omnidirectional }\end{array}$ & 2.245 & TX2 & $\begin{array}{c}r_{B P}=11 \\
\mathrm{~B} 1=-1.1, \mathrm{~A} 1=-46 \\
\mathrm{~B} 2=-2.6, \mathrm{~A} 2=-30\end{array}$ & 58,325 & $\begin{array}{l}\text { Fig. } \\
4-3(a)\end{array}$ & $\begin{array}{l}\text { TX2_2G_Boiler_ } \\
\text { Vpol_run2 }\end{array}$ \\
\hline $\begin{array}{l}\text { RX: Vertical Pol. } \\
\text { TX: Vertical Pol. } \\
\text { Omnidirectional }\end{array}$ & 2.245 & TX2 & $\begin{array}{c}r_{B P}=11 \\
\mathrm{~B} 1=-1.1, \mathrm{~A} 1=-45 \\
\mathrm{~B} 2=-2.5, \mathrm{~A} 2=-32\end{array}$ & 51, 285 & $\begin{array}{l}\text { Fig. } \\
\text { 4-3(a) }\end{array}$ & $\begin{array}{l}\text { TX2_2G_Boiler_ } \\
\text { Vpol_run3 }\end{array}$ \\
\hline $\begin{array}{l}\text { RX: Vertical Pol. } \\
\text { TX: Vertical Pol. } \\
\text { Omnidirectional }\end{array}$ & 2.245 & TX2 & $\begin{array}{c}r_{B P}=11 \\
\mathrm{~B} 1=-1.3, \mathrm{~A} 1=-45 \\
\mathrm{~B} 2=-2.7, \mathrm{~A} 2=-29\end{array}$ & 76, 387 & $\begin{array}{l}\text { Fig. } \\
4-3(a)\end{array}$ & $\begin{array}{l}\text { TX2_2G_Boiler__ } \\
\text { Vpol_run4 }\end{array}$ \\
\hline $\begin{array}{l}\text { RX: Vertical Pol. } \\
\text { TX: Vertical Pol. } \\
\text { Omnidirectional }\end{array}$ & 2.245 & TX2 & $\begin{array}{c}r_{B P}=11 \\
\mathrm{~B} 1=-0.88, \mathrm{~A} 1=-48 \\
\mathrm{~B} 2=-2.3, \mathrm{~A} 2=-36\end{array}$ & 250,528 & $\begin{array}{l}\text { Fig. } \\
\text { 4-3(b) }\end{array}$ & $\begin{array}{l}\text { TX2_2G_Boiler_ } \\
\text { Vpol_rrcos_run3 }\end{array}$ \\
\hline $\begin{array}{l}\text { RX: Vertical Pol. } \\
\text { TX: Vertical Pol. } \\
\text { Horn }\end{array}$ & 2.245 & TX2 & $\begin{array}{c}r_{B P}=11 \\
\mathrm{~B} 1=-0.05, \mathrm{~A} 1=-58 \\
\mathrm{~B} 2=-2.1, \mathrm{~A} 2=-40\end{array}$ & 250,535 & $\begin{array}{l}\text { Fig. } \\
\text { 4-4(a) }\end{array}$ & $\begin{array}{l}\text { TX2_2G_boiler_ } \\
\text { 3115Vpol_run1 }\end{array}$ \\
\hline $\begin{array}{l}\text { RX: Vertical Pol. } \\
\text { TX: Vertical Pol. } \\
\text { Horn }\end{array}$ & 2.245 & TX2 & $\begin{array}{c}r_{B P}=11 \\
\mathrm{~B} 1=0.12, \mathrm{~A} 1=-60 \\
\mathrm{~B} 2=-2.5, \mathrm{~A} 2=-35\end{array}$ & 163,450 & $\begin{array}{l}\text { Fig. } \\
4-4(a)\end{array}$ & $\begin{array}{l}\text { TX2_2G_boiler_ } \\
\text { 3115Vpol_run2 }\end{array}$ \\
\hline $\begin{array}{l}\text { RX: Vertical Pol. } \\
\text { TX: Vertical Pol. } \\
\text { Horn }\end{array}$ & 2.245 & TX3 & $\begin{array}{c}r_{B P}=13 \\
\mathrm{~B} 1=1.8, \mathrm{~A} 1=-84 \\
\mathrm{~B} 2=-3.7 \mathrm{~A} 2=-23\end{array}$ & 132, 370 & $\begin{array}{l}\text { Fig. } \\
\text { 4-4(b) }\end{array}$ & $\begin{array}{l}\text { TX3_2G_boiler_ } \\
\text { 3115Vpol_run2 }\end{array}$ \\
\hline $\begin{array}{l}\text { RX: Vertical Pol. } \\
\text { TX: Vertical Pol. } \\
\text { Omnidirectional }\end{array}$ & 5.4 & TX2 & $\begin{array}{c}r_{B P}=11 \\
\mathrm{~B} 1=-2.5, \mathrm{~A} 1=-37 \\
\mathrm{~B} 2=-2.7, \mathrm{~A} 2=-35\end{array}$ & 204,490 & $\begin{array}{l}\text { Fig. } \\
4-5(a)\end{array}$ & $\begin{array}{l}\text { TX2_5G_Boiler_ } \\
\text { Vpol_run1 }\end{array}$ \\
\hline $\begin{array}{l}\text { RX: Horizontal Pol. } \\
\text { TX: Vertical Pol. } \\
\text { Omnidirectional }\end{array}$ & 5.4 & TX2 & $\begin{array}{c}r_{B P}=11 \\
\mathrm{~B} 1=-2.1, \mathrm{~A} 1=-48 \\
\mathrm{~B} 2=-2.7, \mathrm{~A} 2=-40\end{array}$ & 30,326 & $\begin{array}{l}\text { Fig. } \\
4-5(a)\end{array}$ & $\begin{array}{l}\text { TX2_5G_Boiler_ } \\
\text { Xpol_run1 }\end{array}$ \\
\hline $\begin{array}{l}\text { RX: Vertical Pol. } \\
\text { TX: Vertical Pol. } \\
\text { Horn }\end{array}$ & 5.4 & TX2 & $\begin{array}{c}r_{B P}=11 \\
\mathrm{~B} 1=-2.7, \mathrm{~A} 1=-43 \\
\mathrm{~B} 2=-3.1, \mathrm{~A} 2=-36\end{array}$ & 8, 222 & $\begin{array}{l}\text { Fig. } \\
\text { 4-5(b) }\end{array}$ & $\begin{array}{l}\text { TX2_5G_Boiler_ } \\
\text { 3115Vpol_run1 }\end{array}$ \\
\hline $\begin{array}{l}\text { RX: Horizontal Pol. } \\
\text { TX: Vertical Pol. } \\
\text { Omnidirectional }\end{array}$ & 5.4 & TX2 & $\begin{array}{c}r_{B P}=11 \\
\mathrm{~B} 1=-0.7, \mathrm{~A} 1=-66 \\
\mathrm{~B} 2=-2.7, \mathrm{~A} 2=-45\end{array}$ & 130,419 & $\begin{array}{l}\text { Fig. } \\
\text { 4-5(b) }\end{array}$ & $\begin{array}{l}\text { TX2_5G_Boiler_ } \\
\text { 3115Xpol_run1 }\end{array}$ \\
\hline $\begin{array}{l}\text { RX: Vertical Pol. } \\
\text { TX: Vertical Pol. } \\
\text { Omnidirectional }\end{array}$ & 5.4 & TX3 & $\begin{array}{c}r_{B P}=13 \\
\mathrm{~B} 1=0.33, \mathrm{~A} 1=-73 \\
\mathrm{~B} 2=-3.1, \mathrm{~A} 2=-35\end{array}$ & 20, 296 & $\begin{array}{l}\text { Fig. } \\
\text { 4-6(a) }\end{array}$ & $\begin{array}{l}\text { TX3_5G_Boiler_ } \\
\text { Vpol_run1 }\end{array}$ \\
\hline $\begin{array}{l}\text { RX: Horizontal Pol. } \\
\text { TX: Vertical Pol. } \\
\text { Omnidirectional }\end{array}$ & 5.4 & TX3 & $\begin{array}{c}r_{B P}=13 \\
\mathrm{~B} 1=0.43, \mathrm{~A} 1=-75 \\
\mathrm{~B} 2=-3.3, \mathrm{~A} 2=-33\end{array}$ & 25,317 & $\begin{array}{l}\text { Fig. } \\
4-6(a)\end{array}$ & $\begin{array}{l}\text { TX3_5G_Boiler_ } \\
\text { Xpol_run1 }\end{array}$ \\
\hline $\begin{array}{l}\text { RX: Vertical Pol. } \\
\text { TX: Vertical Pol. } \\
\text { Horn }\end{array}$ & 5.4 & TX3 & $\begin{array}{c}r_{B P}=13 \\
\mathrm{~B} 1=1.3, \mathrm{~A} 1=-87 \\
\mathrm{~B} 2=-3.0, \mathrm{~A} 2=-40\end{array}$ & 204, 507 & $\begin{array}{l}\text { Fig. } \\
\text { 4-6(b) }\end{array}$ & $\begin{array}{l}\text { TX3_5G_3115V } \\
\text { pol_run1 }\end{array}$ \\
\hline $\begin{array}{l}\text { RX: Vertical Pol. } \\
\text { TX: Vertical Pol. } \\
\text { Horn }\end{array}$ & 5.4 & TX3 & $\begin{array}{c}r_{B P}=13 \\
\mathrm{~B} 1=1.2, \mathrm{~A} 1=-86 \\
\mathrm{~B} 2=-3.0, \mathrm{~A} 2=-39\end{array}$ & 32, 317 & $\begin{array}{l}\text { Fig. } \\
\text { 4-6(b) }\end{array}$ & $\begin{array}{l}\text { TX3_5G_3115V } \\
\text { pol_run2 }\end{array}$ \\
\hline $\begin{array}{l}\text { RX: Vertical Pol. } \\
\text { TX: Vertical Pol. } \\
\text { Horn }\end{array}$ & 5.4 & TX3 & $\begin{array}{c}r_{B P}=13 \\
\mathrm{~B} 1=1.3, \mathrm{~A} 1=-87 \\
\mathrm{~B} 2=-3.3, \mathrm{~A} 2=-37\end{array}$ & 35, 328 & $\begin{array}{l}\text { Fig. } \\
\text { 4-6(b) }\end{array}$ & $\begin{array}{l}\text { TX3_5G_3115X } \\
\text { pol_run1 }\end{array}$ \\
\hline
\end{tabular}


Table 4-3: CUP fixed-position runs: omnidirectional = biconical antenna and horn = double-ridged guide antenna.

\begin{tabular}{|c|c|c|c|c|}
\hline $\begin{array}{l}\text { Channel Sounder } \\
\text { Information }\end{array}$ & $\begin{array}{l}\text { Frequency } \\
\text { (GHz) }\end{array}$ & $\begin{array}{c}\text { TX } \\
\text { Position }\end{array}$ & Figure \# & Measurement Filename \\
\hline $\begin{array}{l}\text { RX: Vertical Pol. } \\
\text { TX: Vertical Pol. } \\
\text { Omnidirectional }\end{array}$ & 2.245 & TX2 & $\begin{array}{l}\text { Fig. 4-7(a), } \\
\text { Fig. 4-7(b) }\end{array}$ & Tx2_2G_Boiler_Vpol_CP6_run1 \\
\hline $\begin{array}{l}\text { RX: Vertical Pol. } \\
\text { TX: Vertical Pol. Horn }\end{array}$ & 2.245 & TX2 & $\begin{array}{l}\text { Fig. 4-7(a), } \\
\text { Fig. 4-8(a) }\end{array}$ & Tx2_2G_Boiler_3115Vpol_CP6_run1 \\
\hline $\begin{array}{l}\text { RX: Vertical Pol. } \\
\text { TX: Vertical Pol. Horn }\end{array}$ & 2.245 & TX3 & $\begin{array}{l}\text { Fig. 4-7(a), } \\
\text { Fig. 4-8(b) }\end{array}$ & Tx3_2G_Boiler_3115Vpol_CP6_run2 \\
\hline $\begin{array}{l}\text { RX: Vertical Pol. } \\
\text { TX: Vertical Pol. Horn }\end{array}$ & 5.4 & TX3 & Fig. 4-9 & Tx3_5G_3115Vpol_CP6_run1 \\
\hline $\begin{array}{l}\text { RX: Vertical Pol. } \\
\text { TX: Vertical Pol. } \\
\text { Omnidirectional }\end{array}$ & 2.245 & TX2 & $\begin{array}{l}\text { Fig. 4-10(a), } \\
\text { Fig. 4-10(b) }\end{array}$ & Tx2_2G_Boiler_Vpol_CP8_run2 \\
\hline $\begin{array}{l}\text { RX: Vertical Pol. } \\
\text { TX: Vertical Pol. } \\
\text { Omnidirectional }\end{array}$ & 2.245 & TX2 & $\begin{array}{l}\text { Fig. 4-10(a), } \\
\text { Fig. 4-11(a) }\end{array}$ & Tx2_2G_Boiler_Vpol_CP8_run3 \\
\hline $\begin{array}{l}\text { RX: Vertical Pol. } \\
\text { TX: Vertical Pol. Horn }\end{array}$ & 2.245 & TX2 & $\begin{array}{l}\text { Fig. 4-10(a), } \\
\text { Fig. 4-11(b) }\end{array}$ & Tx2_2G_Boiler_3115Vpol_CP8_run1 \\
\hline $\begin{array}{l}\text { RX: Vertical Pol. } \\
\text { TX: Vertical Pol. Horn }\end{array}$ & 2.245 & TX3 & Fig. 4-12 & Tx3_2G_Boiler_3115Vpol_CP8_run1 \\
\hline $\begin{array}{l}\text { RX: Vertical Pol. } \\
\text { TX: Vertical Pol. } \\
\text { Omnidirectional }\end{array}$ & 2.245 & TX2 & $\begin{array}{l}\text { Fig. 4-13(a), } \\
\text { Fig. 4-13(b) }\end{array}$ & Tx2_2G_Boiler_Vpol_rrcos_CP13_run1 \\
\hline $\begin{array}{l}\text { RX: Vertical Pol. } \\
\text { TX: Vertical Pol. Horn }\end{array}$ & 2.245 & TX2 & $\begin{array}{l}\text { Fig. 4-13(a), } \\
\text { Fig. 4-14(a) }\end{array}$ & Tx2_2G_Boiler_3115Vpol_CP13_run1 \\
\hline $\begin{array}{l}\text { RX: Vertical Pol. } \\
\text { TX: Vertical Pol. Horn }\end{array}$ & 2.245 & TX3 & $\begin{array}{l}\text { Fig. 4-14(b), } \\
\text { Fig. 4-15(a) }\end{array}$ & Tx3_2G_Boiler_3115Vpol_CP13_run1 \\
\hline $\begin{array}{l}\text { RX: Vertical Pol. } \\
\text { TX: Vertical Pol. Horn }\end{array}$ & 5.4 & TX3 & $\begin{array}{l}\text { Fig. 4-15(b), } \\
\text { Fig. 4-16(a) }\end{array}$ & Tx3_5G_3115Vpol_CP13_run1 \\
\hline $\begin{array}{l}\text { RX: Vertical Pol. } \\
\text { TX: Vertical Pol. Horn }\end{array}$ & 5.4 & TX3 & $\begin{array}{l}\text { Fig. 4-16(b), } \\
\text { Fig. 4-17(a) }\end{array}$ & Tx3_5G_3115Vpol_CP18_run1 \\
\hline $\begin{array}{l}\text { RX: Horizontal Pol. } \\
\text { TX: Vertical Pol. Horn }\end{array}$ & 5.4 & TX3 & $\begin{array}{l}\text { Fig. 4-16(b), } \\
\text { Fig. 4-17(b) }\end{array}$ & Tx3_5G_3115Xpol_CP18_run1 \\
\hline $\begin{array}{l}\text { RX: Vertical Pol. } \\
\text { TX: Vertical Pol. Horn }\end{array}$ & 5.4 & TX3 & $\begin{array}{l}\text { Fig. 4-18(a), } \\
\text { Fig. 4-18(b) }\end{array}$ & Tx3_5G_3115Vpol_CP19_run1 \\
\hline $\begin{array}{l}\text { RX: Horizontal Pol. } \\
\text { TX: Vertical Pol. Horn }\end{array}$ & 5.4 & TX3 & $\begin{array}{l}\text { Fig. 4-18(a), } \\
\text { Fig. 4-19(a) }\end{array}$ & Tx3_5G_3115Xpol_CP19_run1 \\
\hline $\begin{array}{l}\text { RX: Vertical Pol. } \\
\text { TX: Vertical Pol. Horn }\end{array}$ & 5.4 & TX3 & $\begin{array}{l}\text { Fig. 4-19(b), } \\
\text { Fig. 4-20(a) }\end{array}$ & Tx3_5G_3115Vpol_CP29_run1 \\
\hline $\begin{array}{l}\text { RX: Vertical Pol. } \\
\text { TX: Vertical Pol. Horn }\end{array}$ & 5.4 & TX3 & $\begin{array}{l}\text { Fig. 4-19(b), } \\
\text { Fig. 4-20(b) }\end{array}$ & Tx3_5G_3115Vpol_CP33_run1 \\
\hline
\end{tabular}


The measurement campaign for the CUP industrial steam plant includes mobile and fixed measurements. The route for the mobile measurements went between the large boilers in the facility on the large loop. The loop route went past multiple reflective metals, six large boilers with many overhead pipes and conduits. Also, while one side of the boiler floor had a large cinder block wall, the opposite wall was a large glass window wall with visibility to the outside. This glass wall is located near the check points 5, 6, 15, 19, 20, and 33. Check points 1, 2, 7, 9, 10, 11, and 23 had an almost direct Line-of-Sight (LOS) to the transmitter TX2 while check points 14 would have the greatest Non-Line-of-Sight (nLOS) components. Table 4-2 contains a list of the measurement runs and names for the mobile measurements.

The mobile CUP measurements consist of multiple identical measurements for different transmitter positions, transmit antenna types, and center frequencies. The first set of mobile measurements used the TX2 position and a Biconical ("omnidirectional”) transmit antenna at the center frequency of $2.245 \mathrm{GHz}$ for path gain. Results are seen in Fig. 4-3. For a single measurement run, a raised-cosine filter was applied digitally to the TX channel sounder signal in order to reduce the Internal Symbol Interference (ISI). The results are shown in Fig. 4-3(b). Using a two-slope polynomial fit, we performed a linear regression on the path gain results with a break point at 11 or $13 \mathrm{~m}$ for all measurement runs. This break point is kept the same for all of the similar dimensional campaign routes in this document [13]. This allows for ease of comparison over all of the measurement campaigns. The slope of the linear regression fit can be found in Table 4-2. For a break point of $11 \mathrm{~m}$, the slopes, B2, of these measurements are similar for similar measurement runs $(-2.6 \mathrm{~dB} / \mathrm{m},-2.5 \mathrm{~dB} / \mathrm{m}$, and $-2.7 \mathrm{~dB} / \mathrm{m})$. These are similar to values reported in $[14,15]$. The slopes are somewhat larger than the free space slope of $2 \mathrm{~dB} / \mathrm{m}$ caused by an increase due to multipath effects.

Fig. 4-4(a) contains the path gain for transmitter position TX2 with the double-ridged guide ("horn”) TX antenna at a center frequency of $2.245 \mathrm{GHz}$. The slopes are $-2.1 \mathrm{~dB} / \mathrm{m}$ and $-2.5 \mathrm{~dB} / \mathrm{m}$ for two similar runs assuming a break point of $11 \mathrm{~m}$. In contrast, Fig. 4-4(b) contains the path gain for the TX3 position, with the horn transmit antenna at the center frequency of $2.245 \mathrm{GHz}$. The slope for this figure is $-3.7 \mathrm{~dB} / \mathrm{m}$. This dramatic change from $-2.5 \mathrm{~dB} / \mathrm{m}$ to $-3.7 \mathrm{~dB} / \mathrm{m}$ indicates that the position of the transmitter antenna is critical to the placement of the wireless access point. In this case, the TX antenna on the upper floor interacted more with pipes and other reflective objects on the ceiling, causing additional multipath.

The path gain slopes at the center frequency of $5.4 \mathrm{GHz}$ are all comparable, between $-2.7 \mathrm{~dB} / \mathrm{m}$ to $-3.3 \mathrm{~dB} / \mathrm{m}$ after the break point of 11 or $13 \mathrm{~m}$, as shown in Fig. 4-5. However, before the break point, the slopes are dramatically different. The slope for TX2 is around $-2.1 \mathrm{~dB} / \mathrm{m}$ to $-2.7 \mathrm{~dB} / \mathrm{m}$ except for the Xpol case, which had a slope of $-0.7 \mathrm{~dB} / \mathrm{m}$. Meanwhile for the TX3 transmitter position, the slopes are different than the TX2 transmitter position. These slopes range from 0.33 to $1.3 \mathrm{~dB} / \mathrm{m}$ [14]. These slopes represent ranges distances of less than $11 \mathrm{~m}$. From Eq. 2.25, we see that the path gain values are computed by subtracting a bulk antenna gain from the PDPs, thus ignoring any antenna radiation pattern influences. As the range decreases, these antenna radiation patterns will have greater effects on the power being received by the channel sounder, since the signal may be propagating strongly into off-angle radiation pattern values. Current research being conducted at the NIST Communication Technology Laboratory is analyzing these effects and providing a traceable uncertainty analysis for proper handling of the antenna radiation pattern 
effects on the path gain. Thus, the approach for a bulk antenna gain may lead to small slope value at small range values.

The shapes of the path gain plots are depend directly upon the position of the transmitter location and not the center frequency or antenna type. Specifically, at position TX2, the path gains are linear with nearly identical slopes through the entire plots. At position TX3, the path gains have a "knee" in the graphs. This "knee" usually occurs at $13 \mathrm{~m}$ independent of the frequency and antenna type. The "knee" depends upon the configuration of the antenna position relative to the environment. The TX3 as mentioned before, is placed high up near the piping and conduits close to the ceiling. Therefore, the radiation from the antenna may be trapped in a waveguide geometry for the first $13 \mathrm{~m}$ before it can begin to drop down into the boiler floor. Once the radiation gets to the boiler floor than it begins to have a typical $\sim-3 \mathrm{~dB} / \mathrm{m}$ slope.

The CUP steam plant also had many fixed-position measurement runs as described in Section 2.3.2. The check points were $6,8,13,18,19,29$, 33. As seen from Fig. 4-2, check points 8 and 29 have a direct LOS to the transmitter position TX2 and all other check points for the fixedposition measurement runs do not. The path gain plots are presented in two ways for the fixedposition measurement runs. The first method shows the path gain with error bars versus range (i.e., distance from the TX antenna). The error bars are computed over the range of the antenna positioner. The second method shows the path gain versus electrical wavelength based upon the check point. Therefore, there is no range between the TX and RX antenna involved with the second method.

The fixed-position measurement runs provide insight into the fast fading that an industrial environment may exhibit. While there is a wealth of information and data here, two points stand out. The first point can be seen in Fig. 4-7(a) and Fig. 4-10(a), which show path gain versus check points 6 and 8, respectively. For the case where the TX and RX antennas are on the same level, check point 6 is in a NLOS condition but in Fig. 4-7(a) the omnidirectional transmit antenna exhibits greater path gain than the horn transmit antenna. For check point 8, which is in a LOS condition, the horn transmit antenna has more path gain than the omnidirectional transmit antenna. This makes physical sense because the RX antenna is in direct line to the radiation from the TX antenna, while at check point 6 because the horn was not reoriented as the RX moves, the majority of the radiation from the horn antenna will not propagate in the direction of check point 6 . Conversely, for an omnidirectional antenna, the radiation is distributed in multiple directions so it has a higher probability to reach check point 6.

The second point deals with the vertical polarization versus horizontal polarization for the RX antenna. In Fig. 4-16(b), the path gain for the transmitter position TX3 for the $5.4 \mathrm{GHz}$ center frequency is compared for the different polarizations. The vertical polarization $\mathrm{Rx}$ antenna measurements have less path gain than the horizontal polarized receiver antenna measurements. Now contrast these results to Fig. 4-19(a) where the vertically and horizontally polarized RX antenna measurements have more comparable results. Clearly, further investigation into polarization diversity is warranted although, as seen through these results, the vertically polarized $\mathrm{Rx}$ antenna measurements usually have less path gain than the other polarized $\mathrm{Rx}$ antenna measurements. 


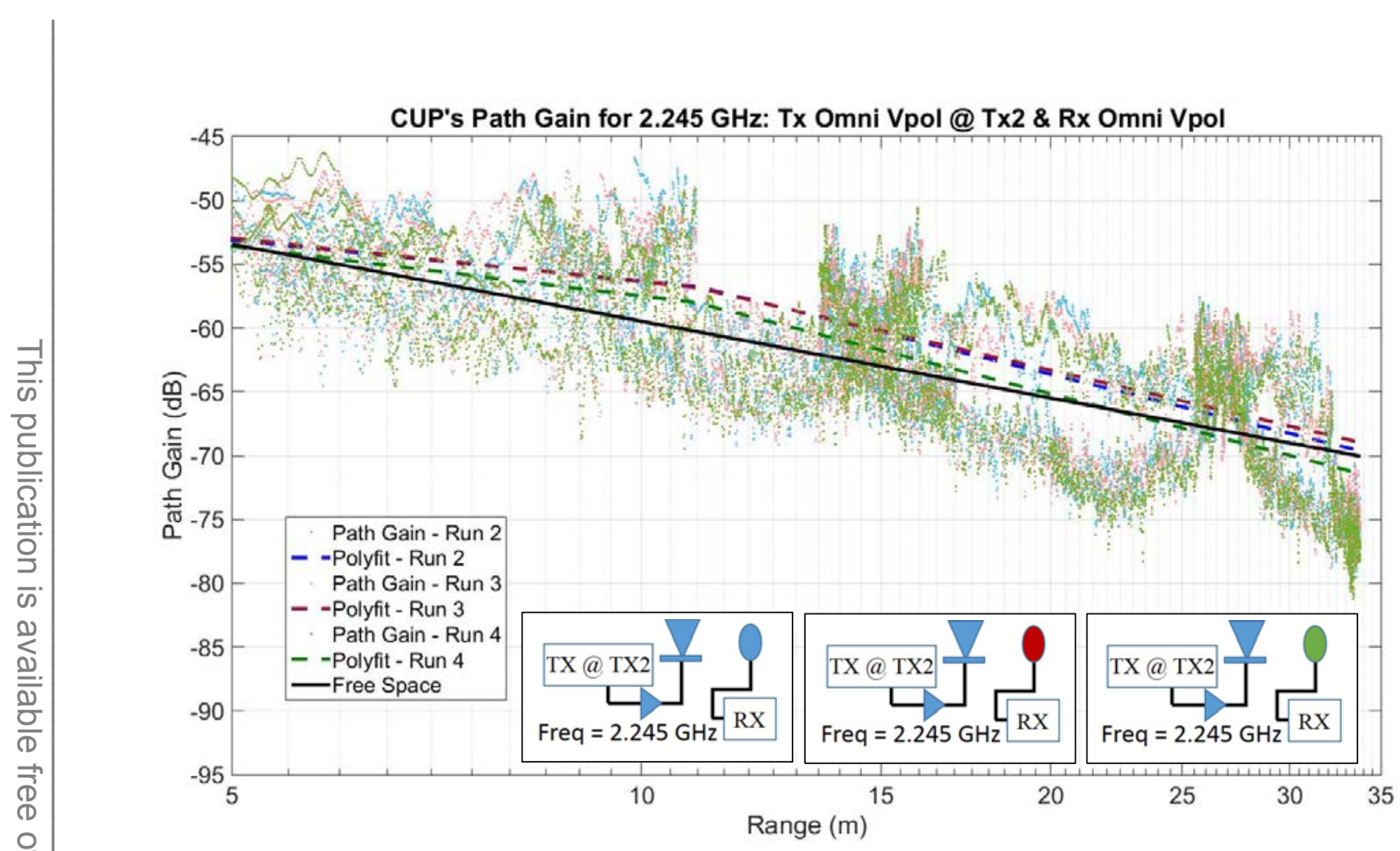

(a)

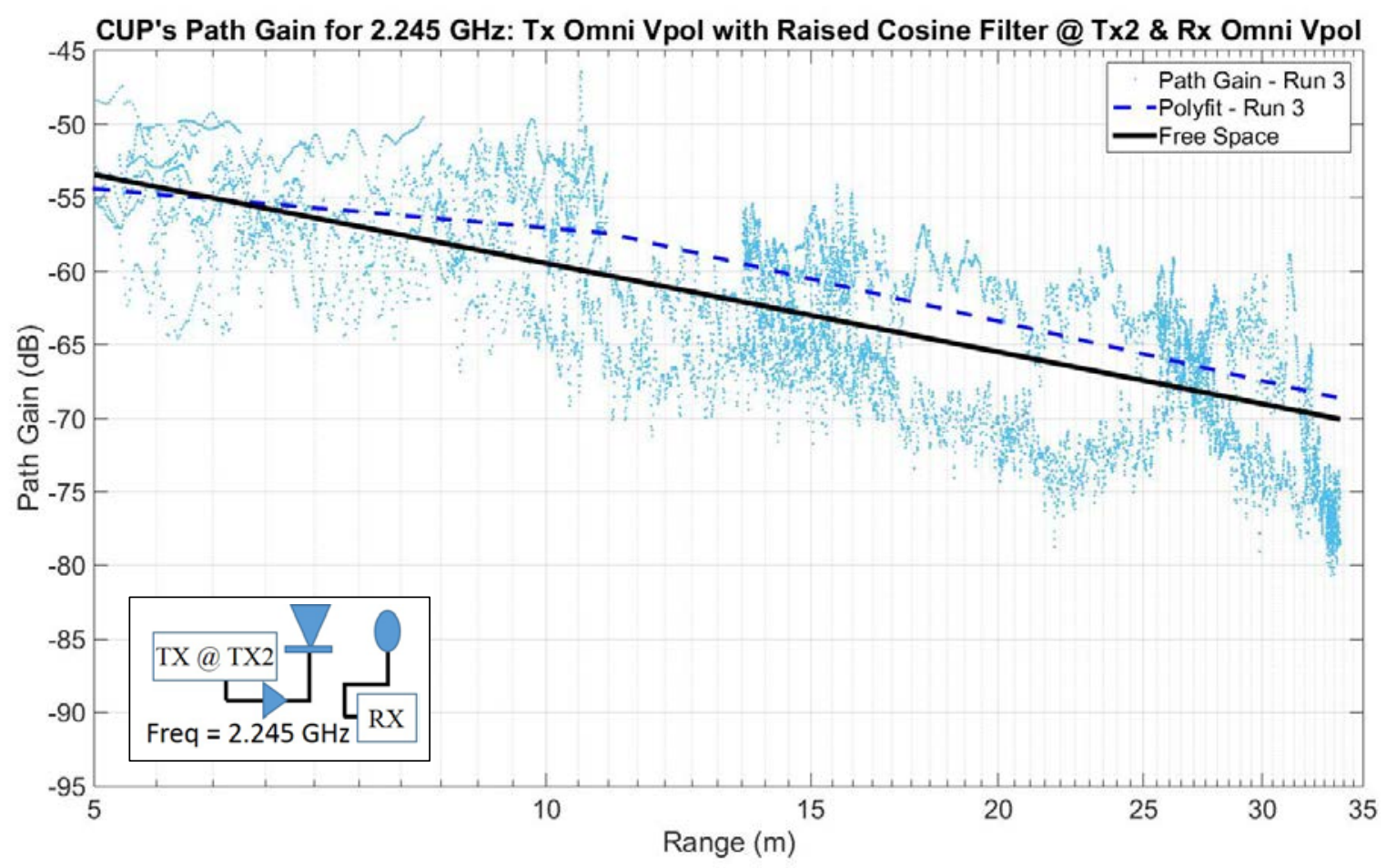

(b)

Fig. 4-3 Path gain measurements in the NIST Central Utility Plant (CUP) for $2.245 \mathrm{GHz}$ (a) Vpol omnidirectional TX antenna at position TX2 and Vpol omnidirectional RX antenna and (b) raised cosine filter applied to the TX signal for the same antenna configuration. 


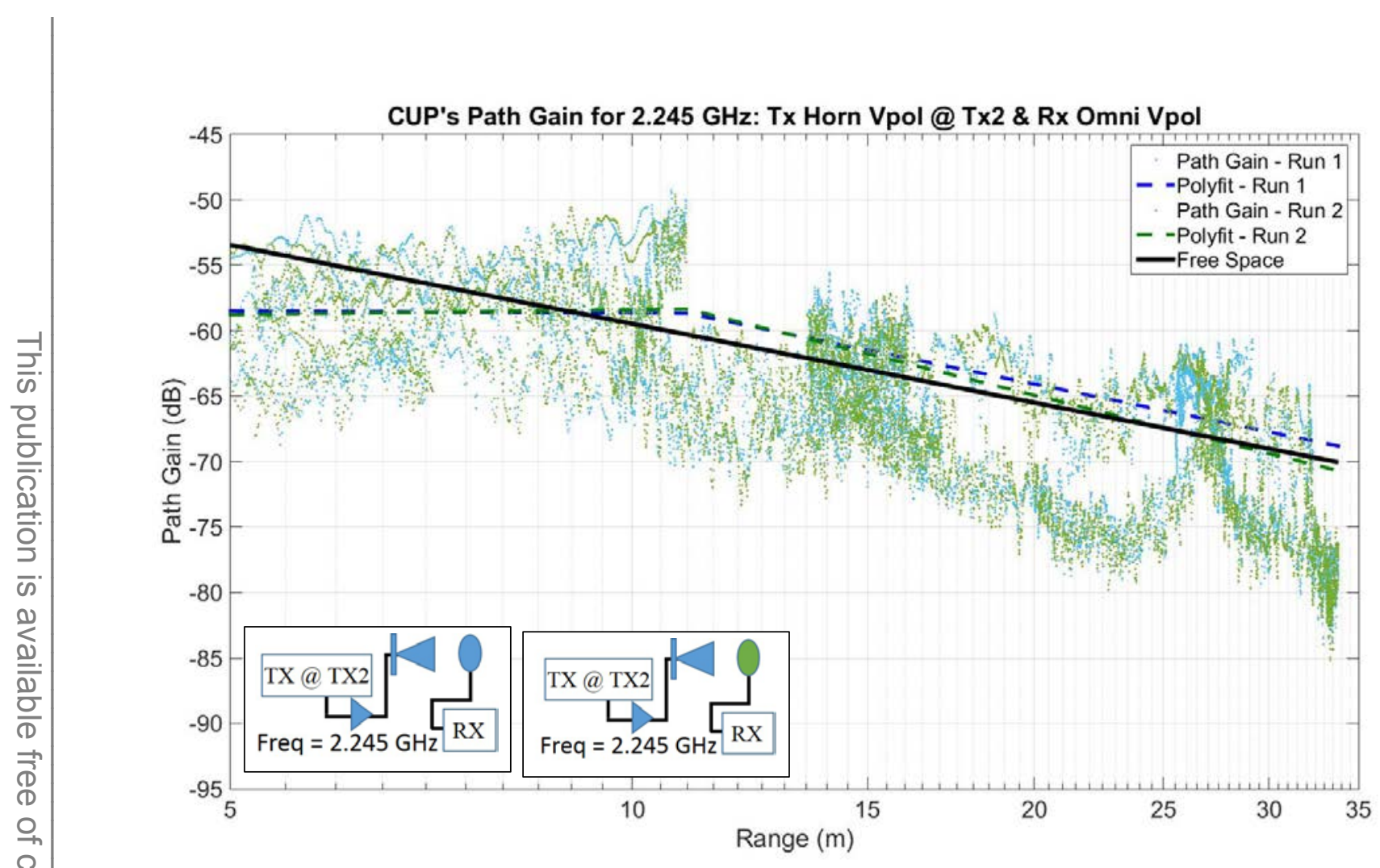

(a)

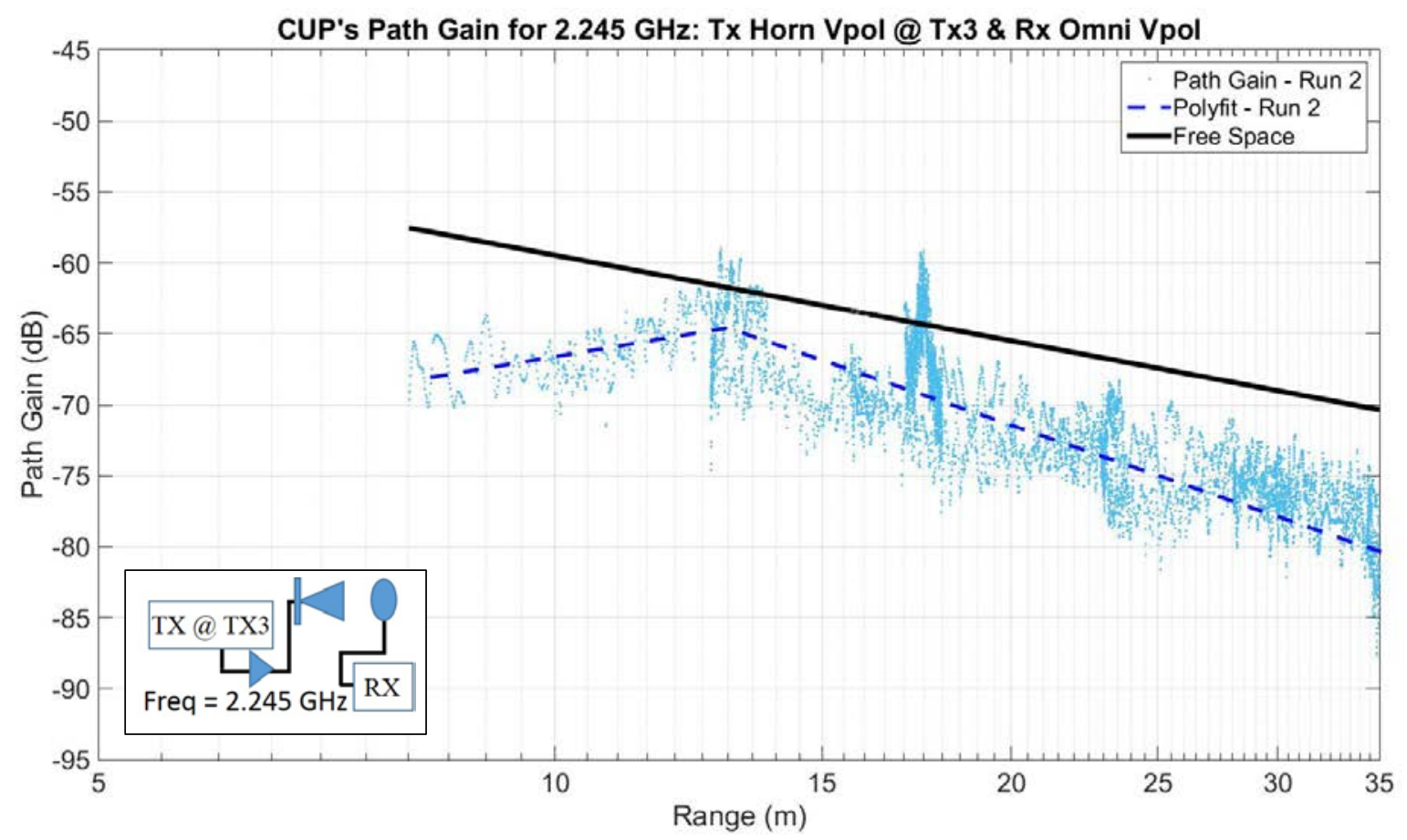

(b)

Fig. 4-4 Path gain measurements in the NIST CUP for 2.245 GHz Vpol horn TX antenna and Vpol omnidirectional RX antenna at (a) position TX2 and (b) position TX3. 


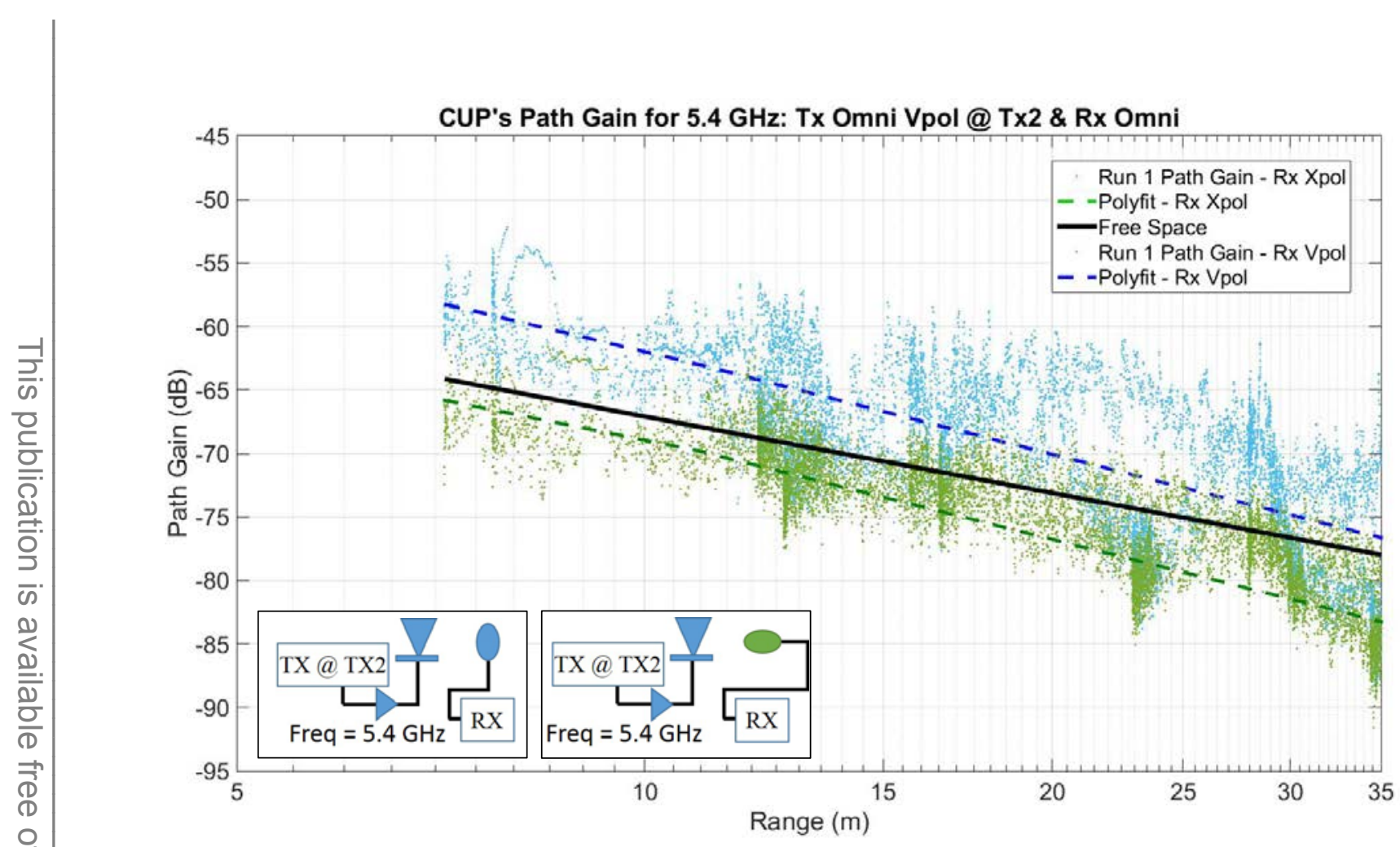

(a)

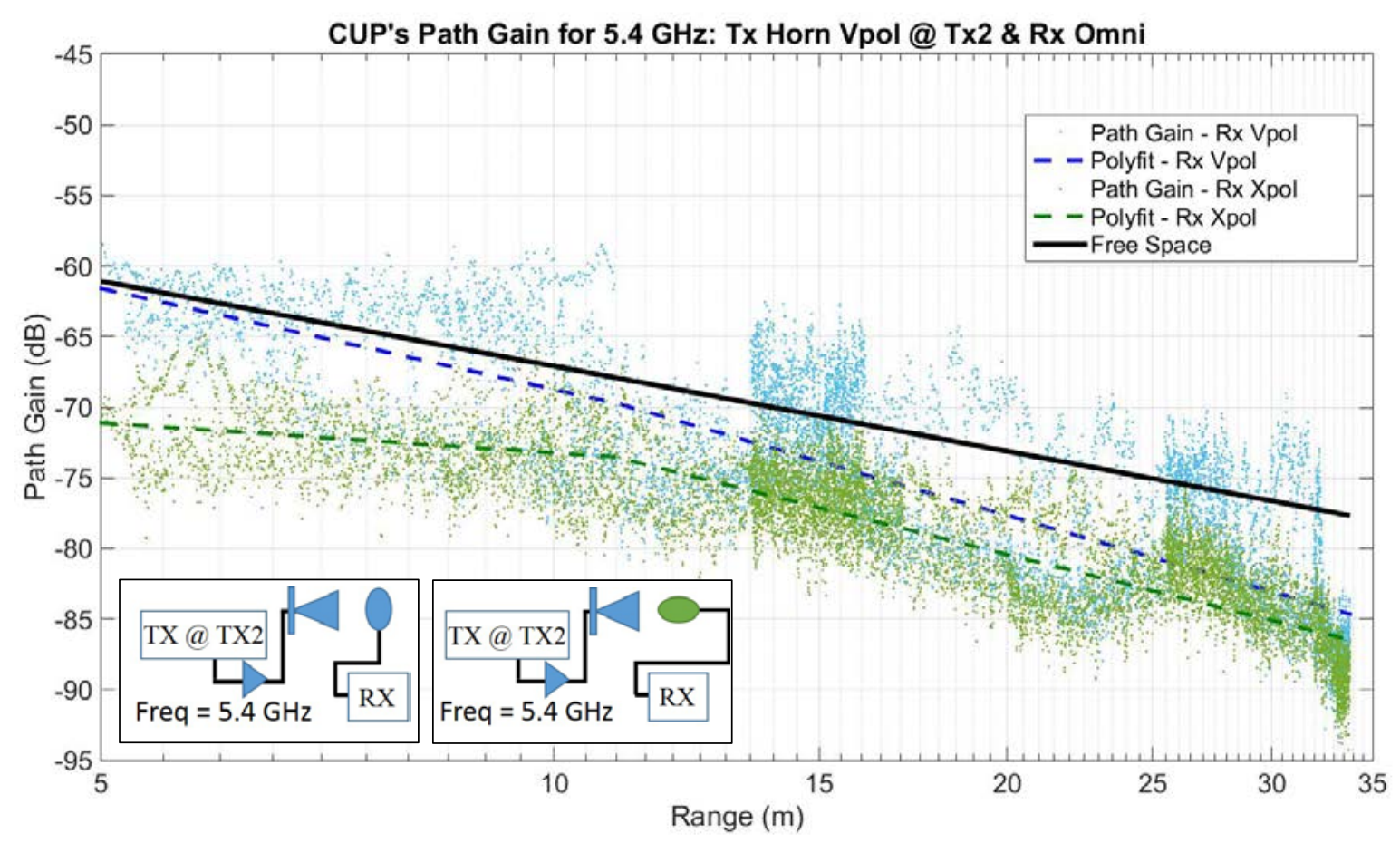

(b)

Fig. 4-5 Path gain measurements in the NIST CUP at 5.4 GHz for TX antenna and Vpol or Xpol polarized RX omnidirectional antenna at (a) Vpol omnidirectional TX antenna position TX2 and (b) Vpol horn position TX2. 


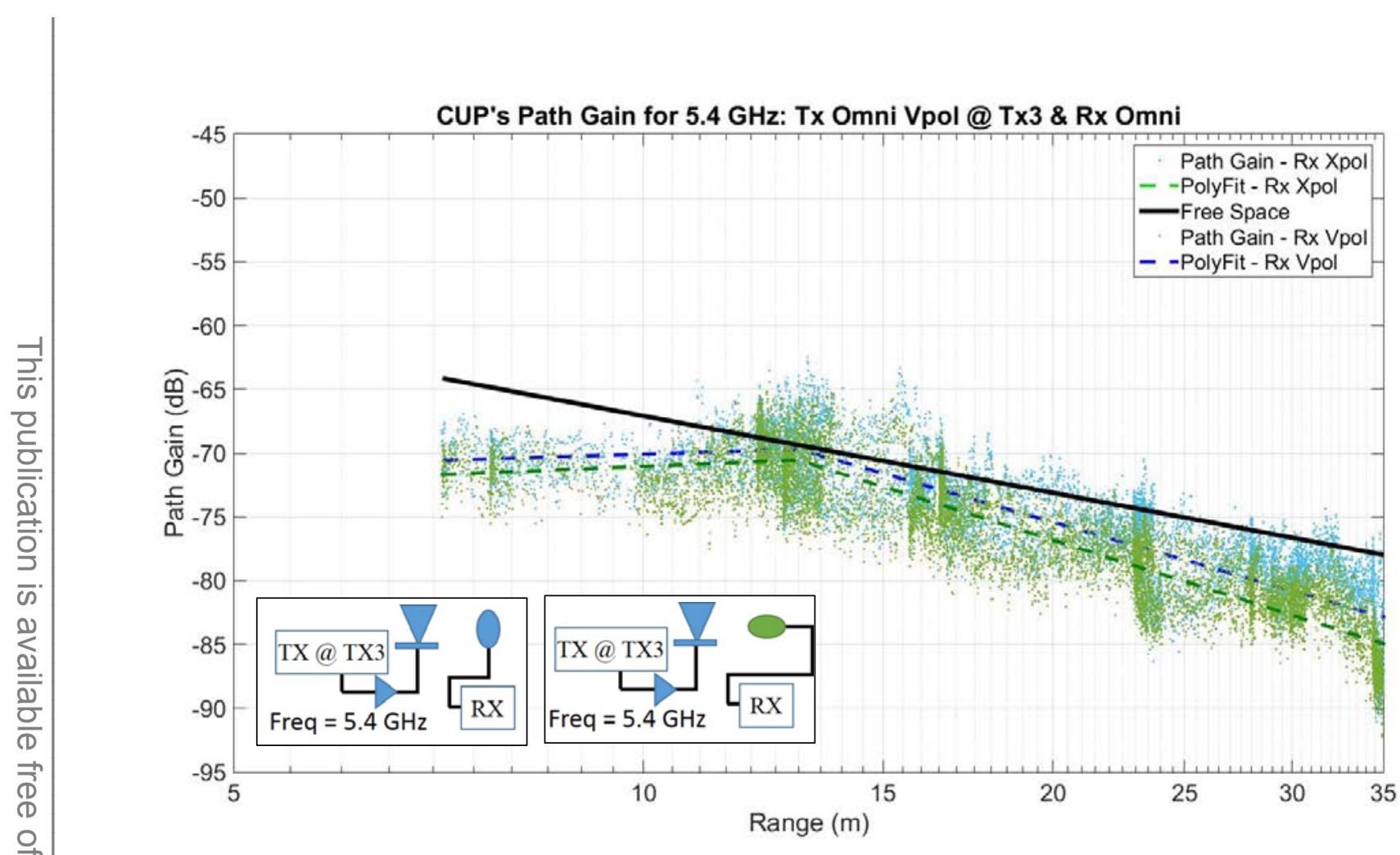

(a)

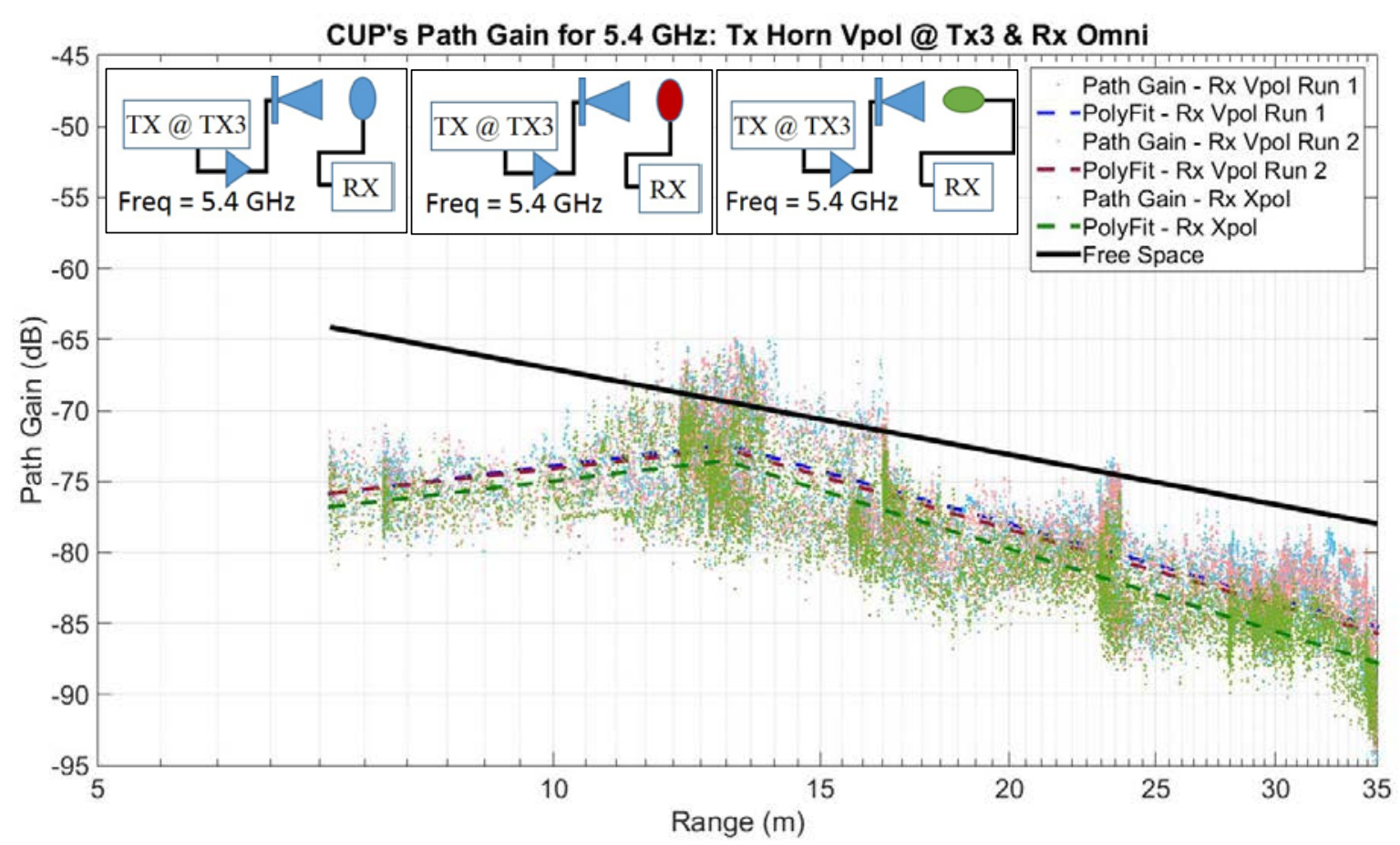

(b)

Fig. 4-6 Path gain measurements in the NIST CUP at $5.4 \mathrm{GHz}$ for TX antenna at position TX3 for Vpol or Xpol omnidirectional RX antenna and (a) Vpol omnidirectional TX antenna and (b) Vpol TX antenna. 


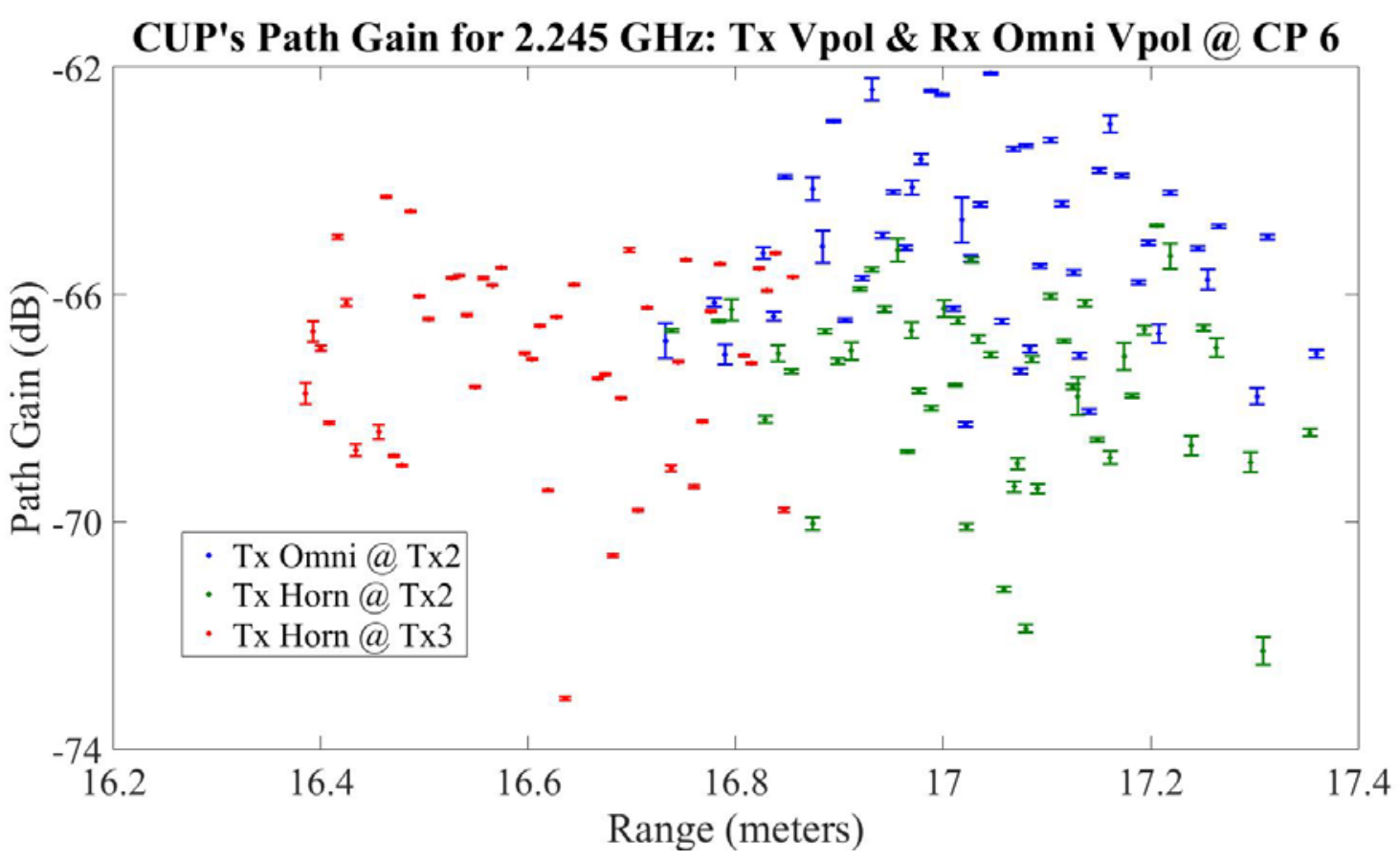

(a)

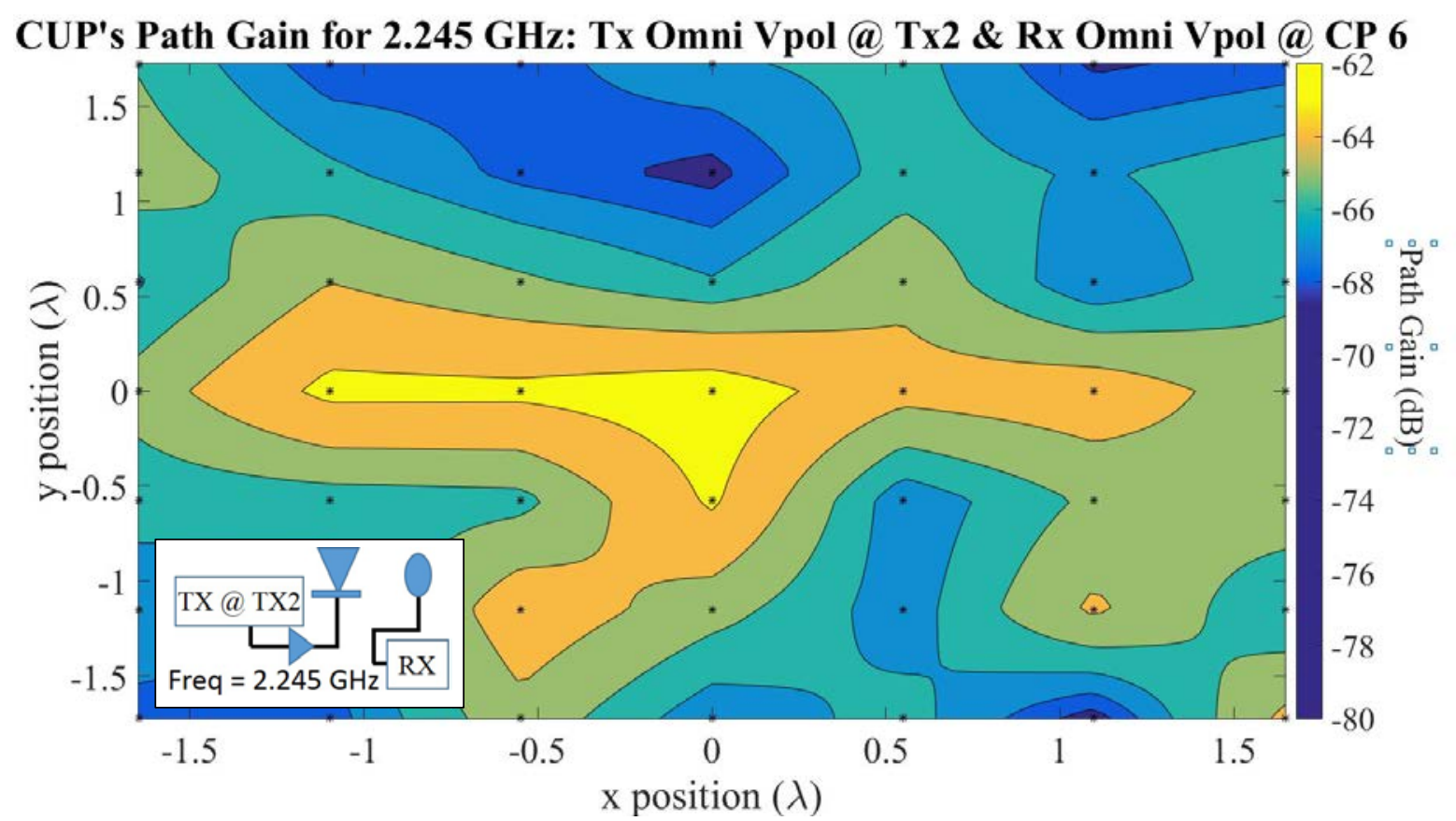

(b)

Fig. 4-7 Path gain measurements in the NIST CUP at 2.245 GHz for Vpol TX antenna at position TX2 or TX3 and Vpol omnidirectional RX antenna at check point 6: (a) range plot and (b) contour plot with omnidirectional TX antenna at check point 6. 


\section{CUP's Path Gain for 2.245 GHz: Tx Horn Vpol @ Tx2 \& Rx Omni Vpol @ CP 6}

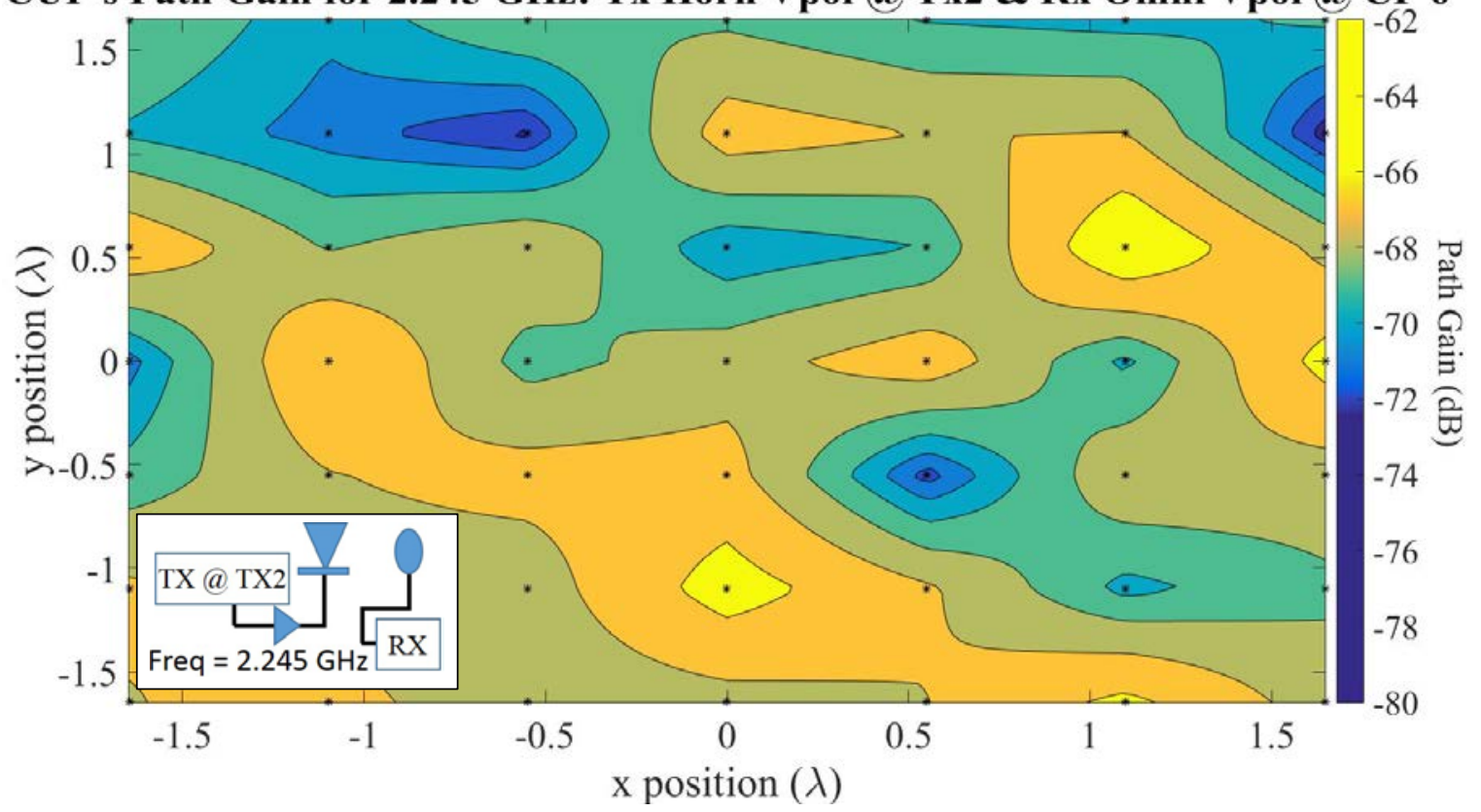

(a)

\section{CUP's Path Gain for 2.245 Ghz: Tx Horn Vpol@Tx3 \& Rx Omni Vpol@ CP 6}

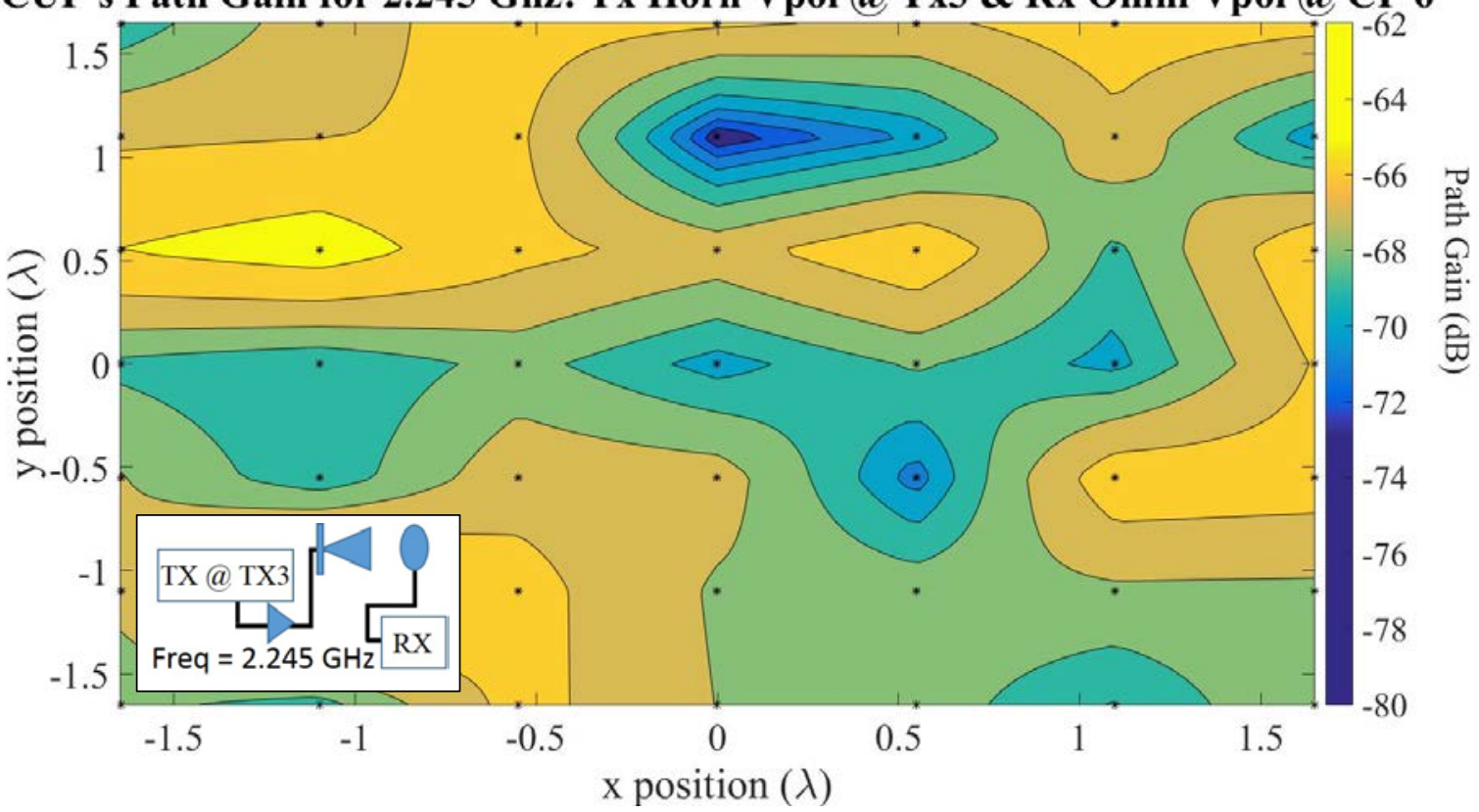

(b)

Fig. 4-8 Path gain measurements in the NIST CUP at $2.245 \mathrm{GHz}$ for Vpol horn TX antenna and Vpol omnidirectional RX antenna at check point 6: (a) at position TX2 and (b) at position TX3. 


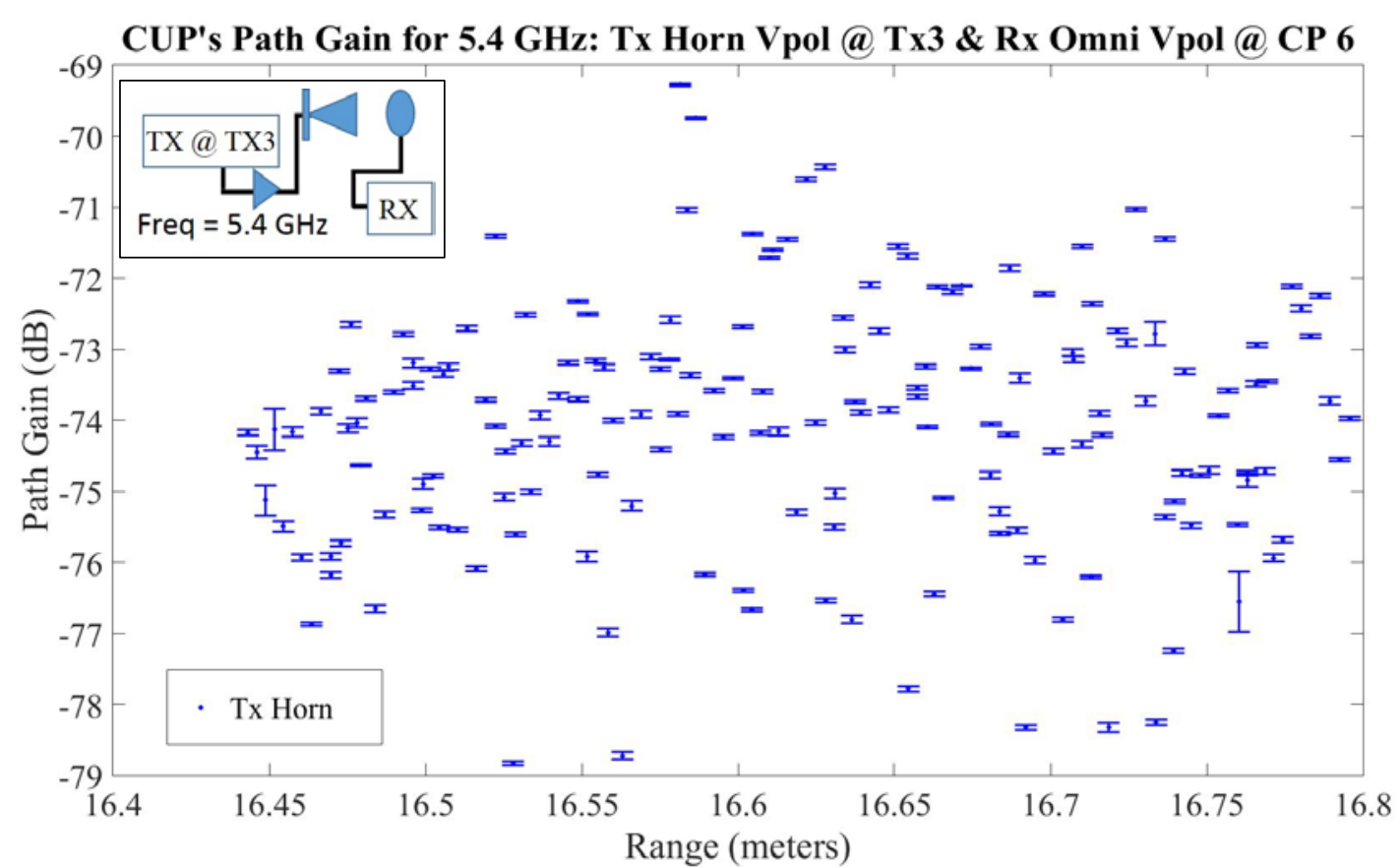

(a)

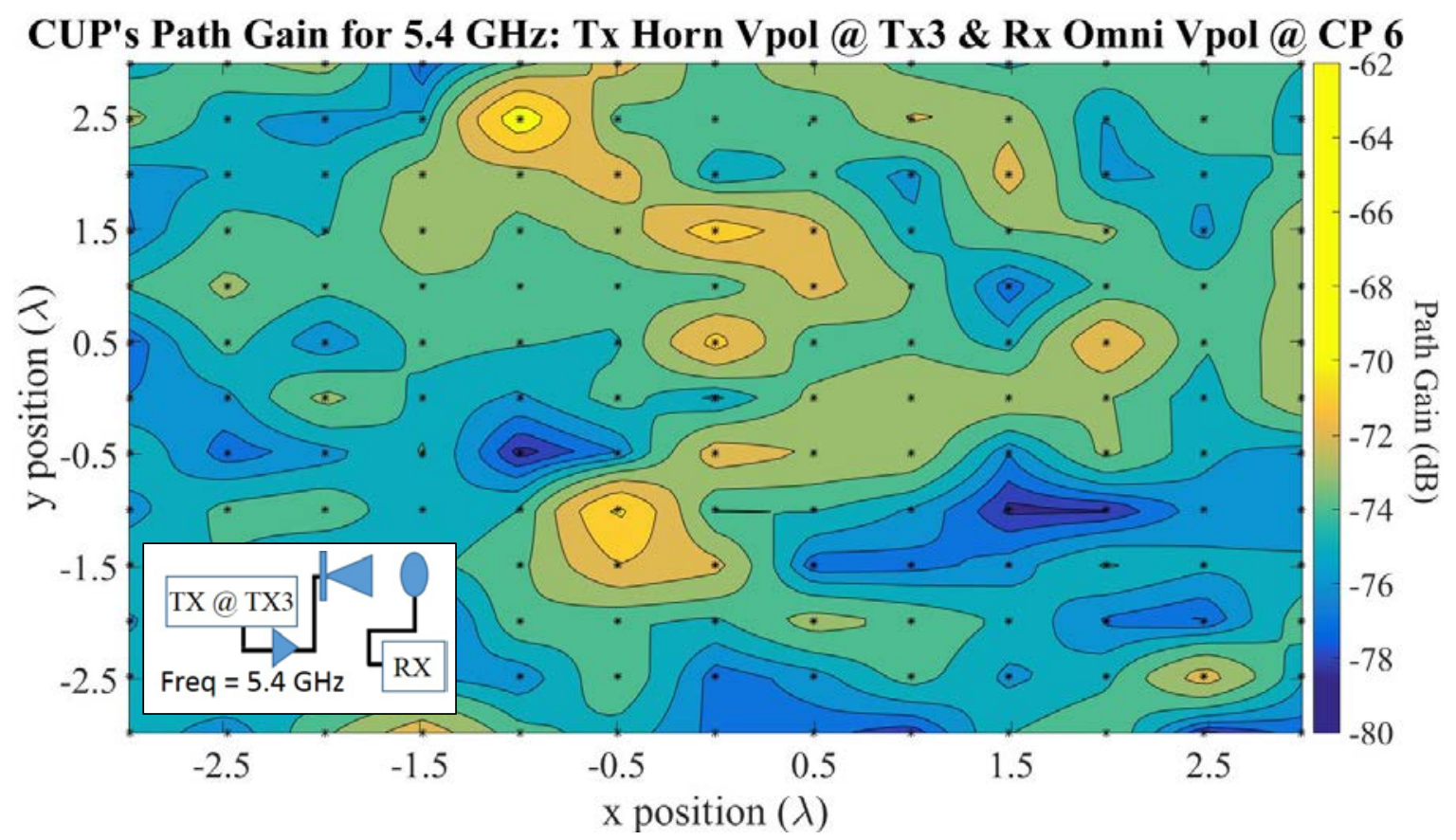

(b)

Fig. 4-9 Path gain measurements in the NIST CUP at 5.4 GHz for Vpol horn TX antenna at position TX3 and Vpol omnidirectional RX antenna at check point 6: (a) range plot and (b) contour plot. 


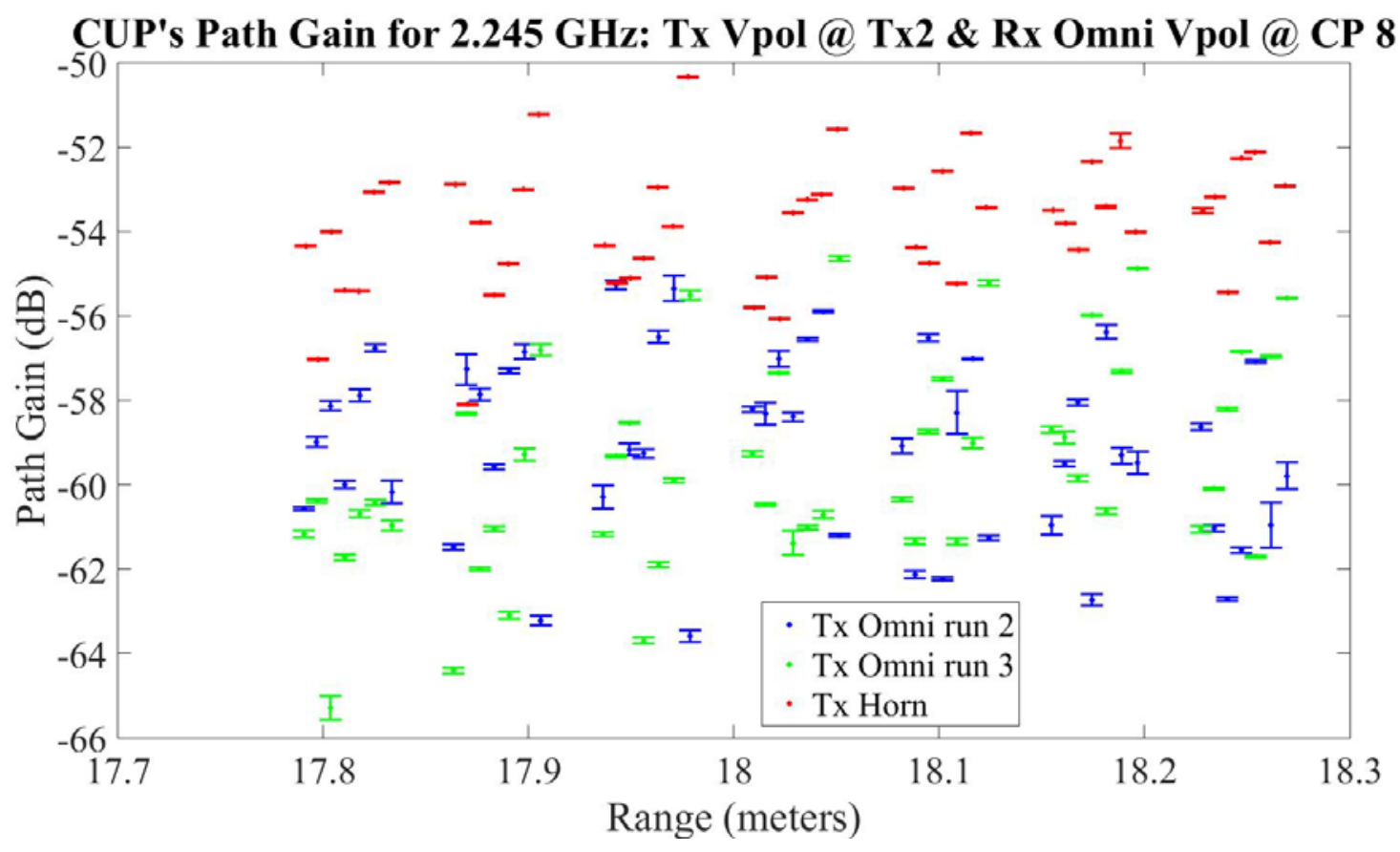

(a)

\section{CUP's Path Gain for 2.245 GHz Run 2:Tx Omni Vpol@ Tx2 \& Rx Omni Vpol @ CP 8}

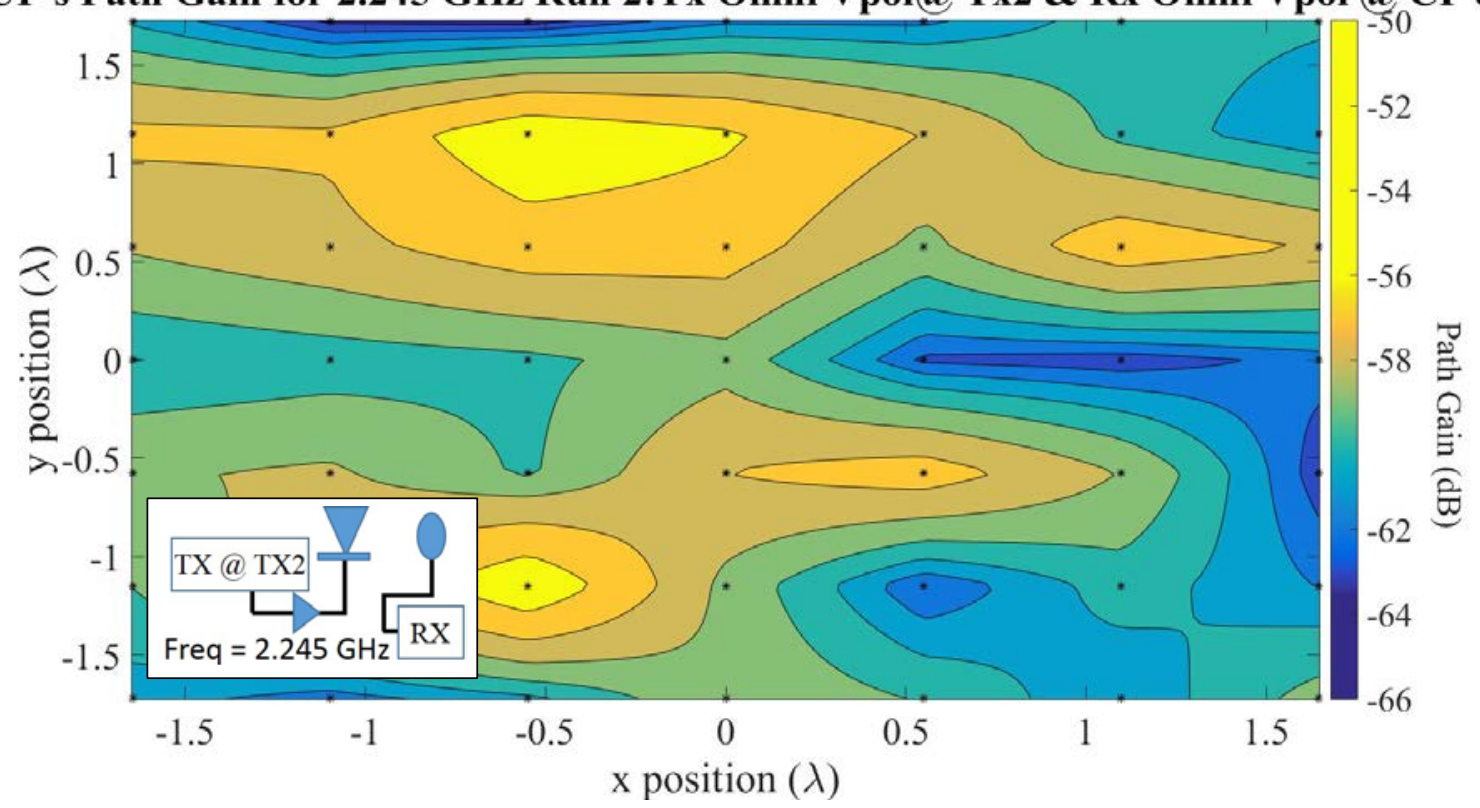

(b)

Fig. 4-10 Path gain measurements in the NIST CUP at $2.245 \mathrm{GHz}$ for Vpol horn or omnidirectional TX antenna at position TX2 and Vpol omnidirectional RX antenna at check point 8: (a) range plot and (b) contour plot with omnidirectional TX antenna. 


\section{CUP's Path Gain for 2.245 GHz Run 3:Tx Omni Vpol@ Tx2 \& Rx Omni Vpol @CP 8}

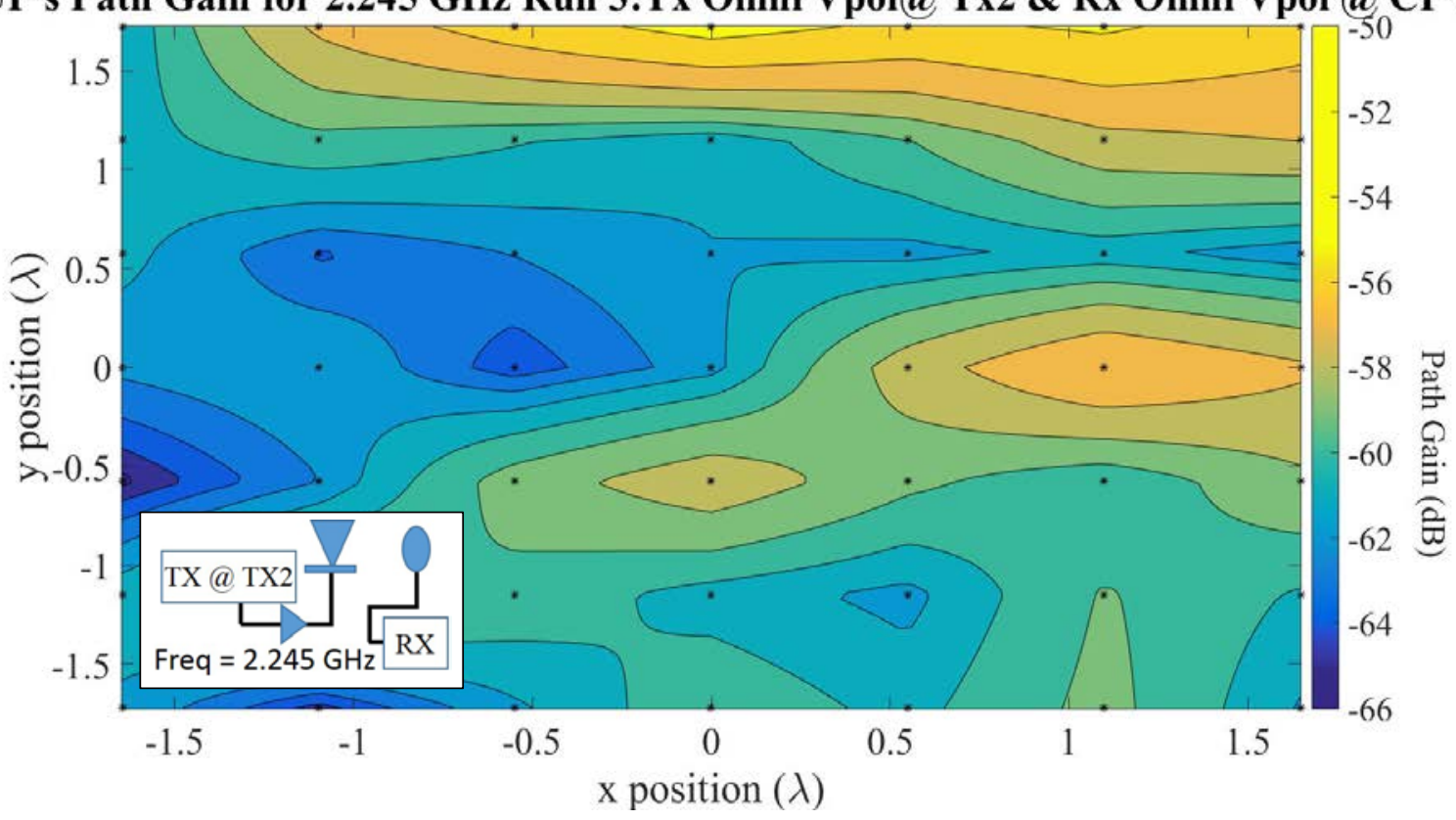

(a)

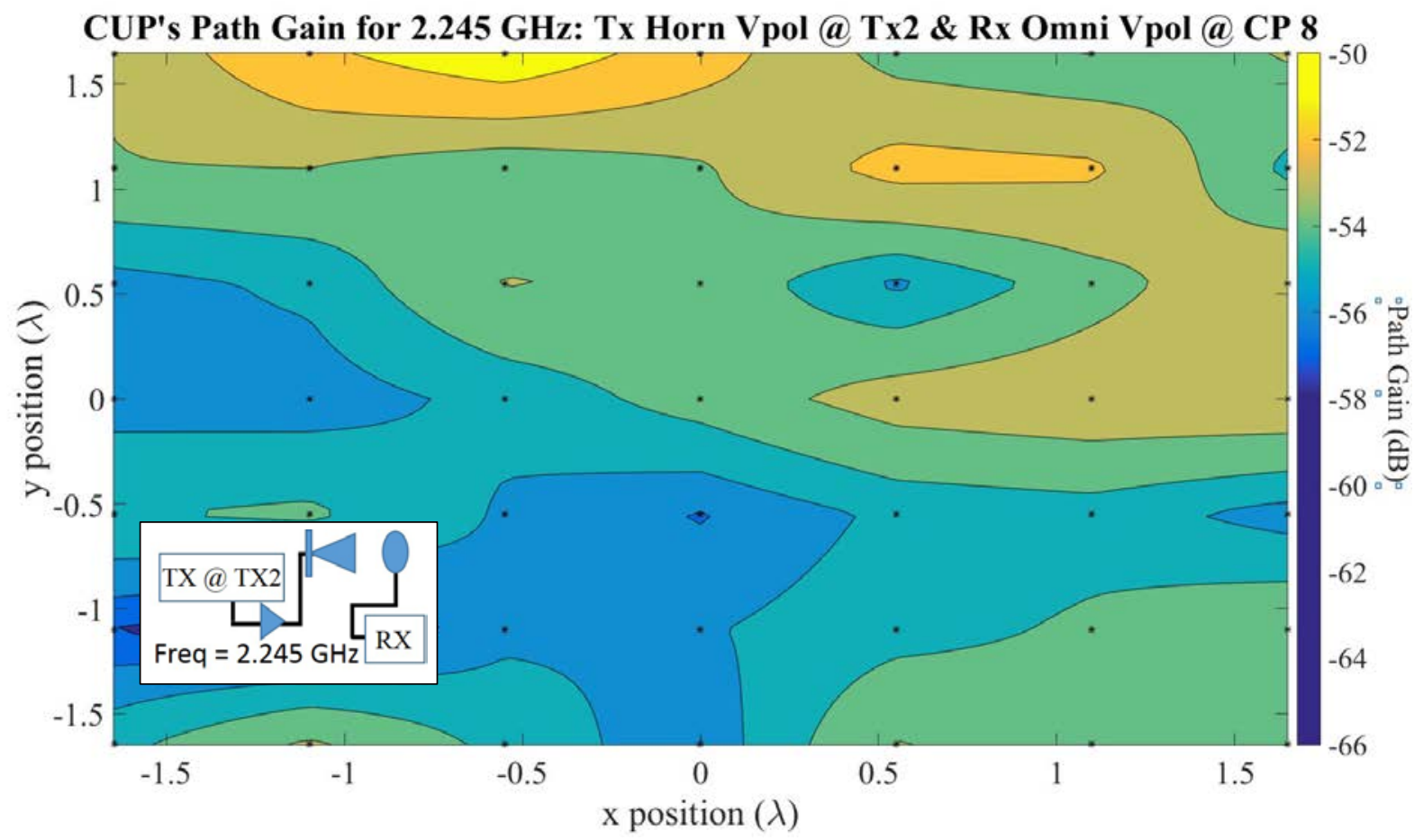

(b)

Fig. 4-11 Path gain measurements in the NIST CUP at 2.245 GHz for Vpol TX antenna at position TX2 and Vpol omnidirectional RX antenna at check point 8: (a) omnidirectional TX antenna and (b) horn TX antenna. 


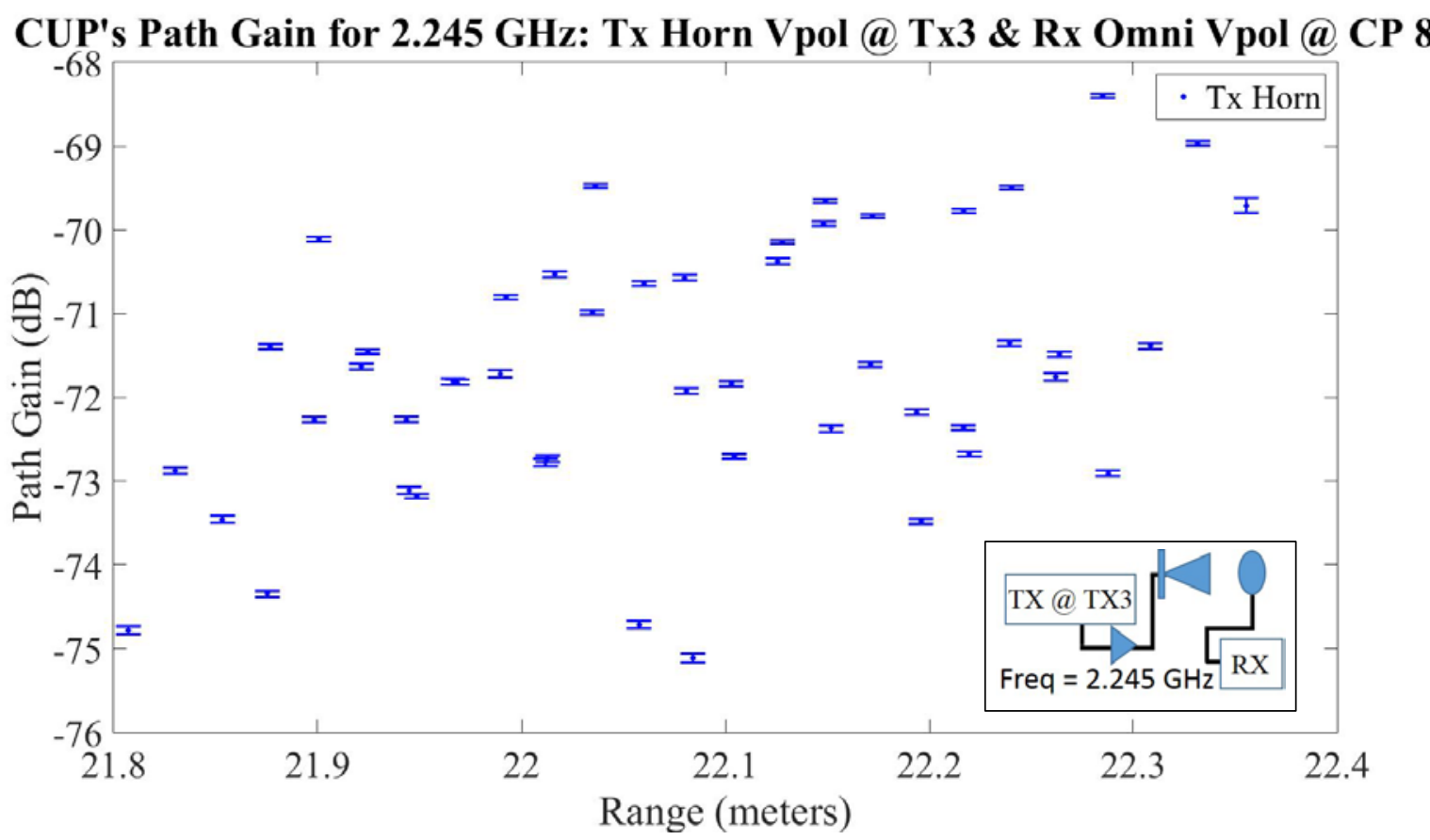

(a)

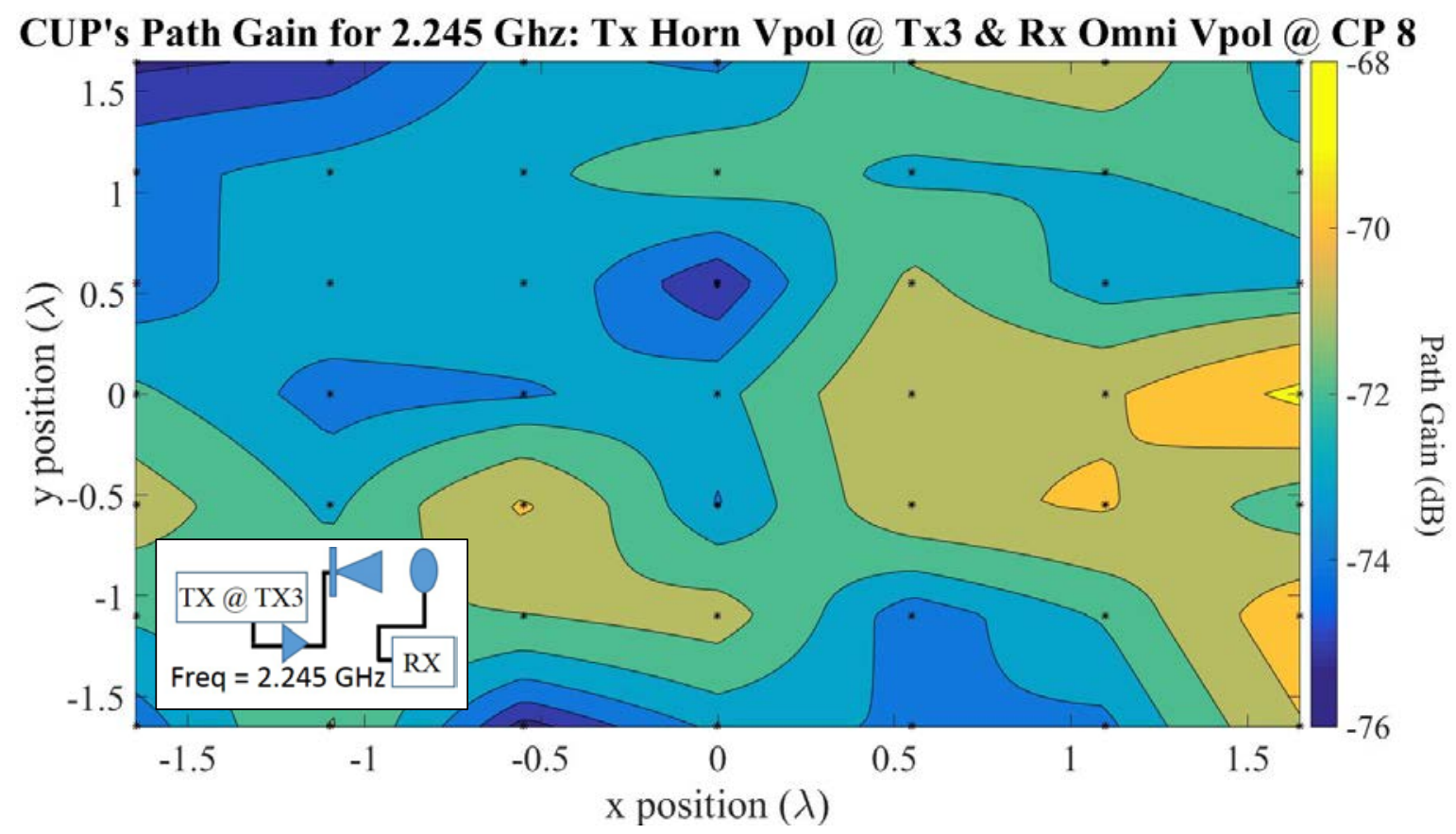

(b)

Fig. 4-12 Path gain measurements in the NIST CUP at $2.245 \mathrm{GHz}$ for Vpol horn TX antenna at position TX3 and Vpol omnidirectional RX antenna at check point 8: (a) range plot and (b) contour plot. 


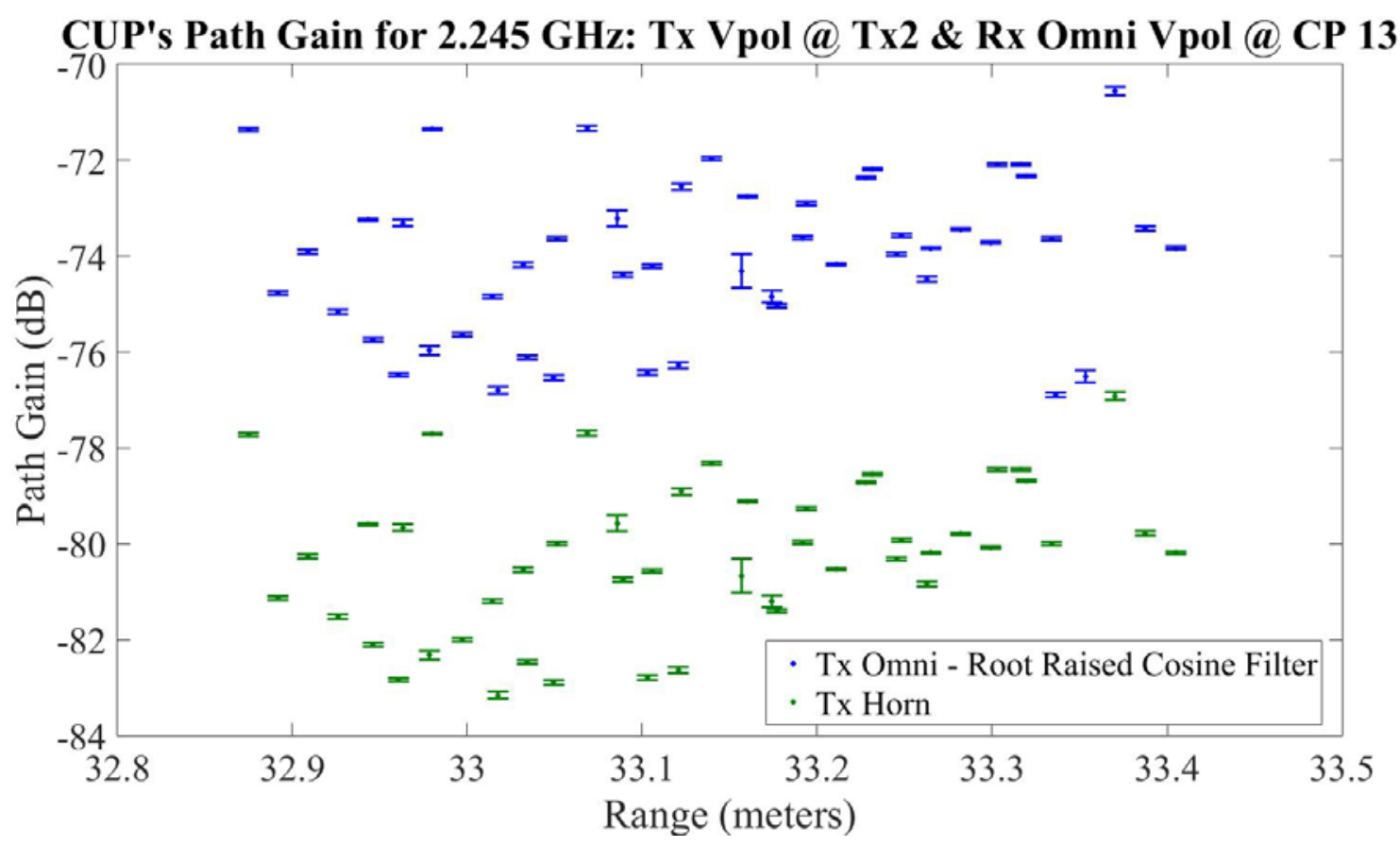

(a)

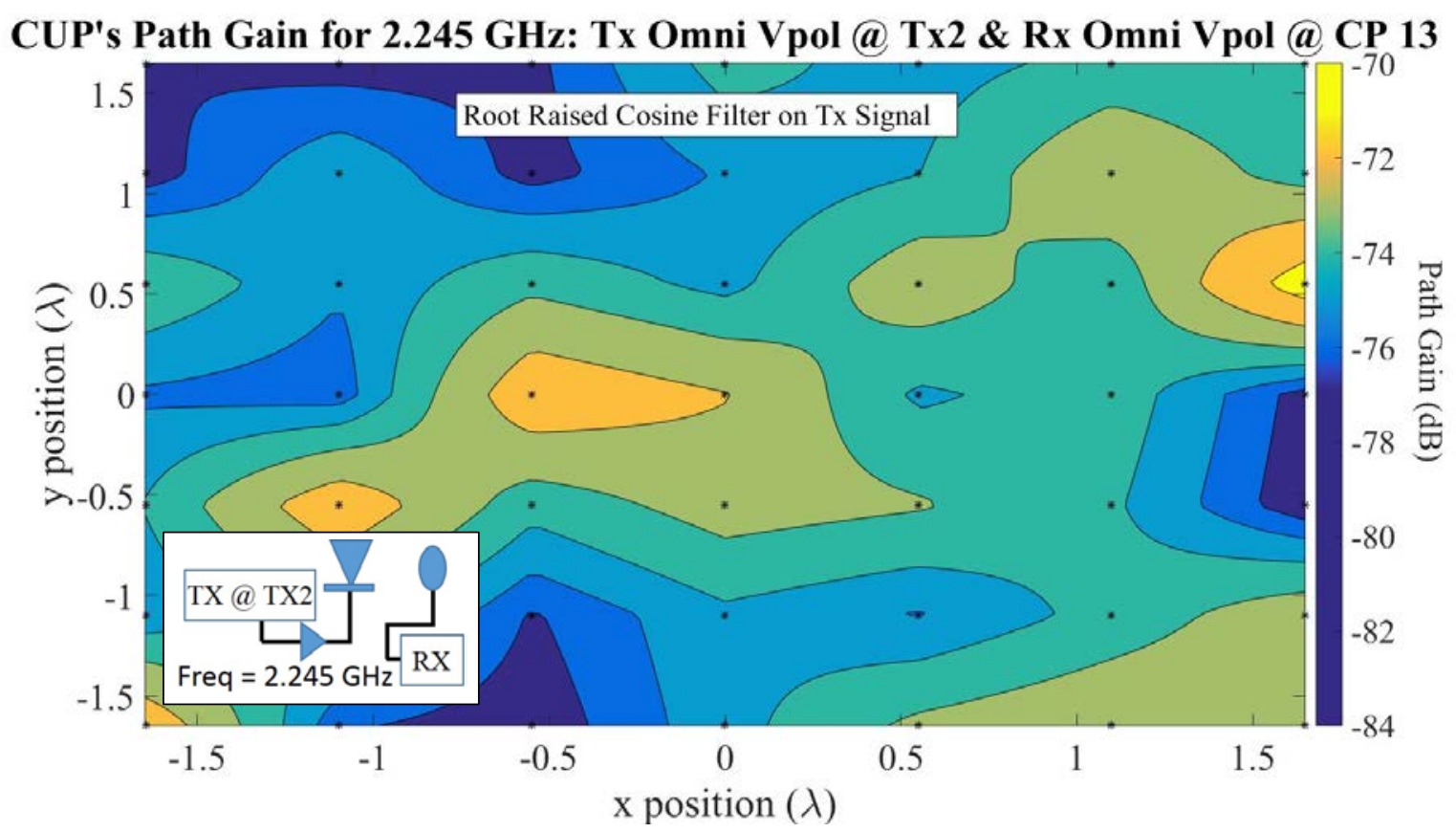

(b)

Fig. 4-13 Path gain measurements in the NIST CUP at $2.245 \mathrm{GHz}$ for Vpol horn or omnidirectional TX antenna at position TX2 and Vpol omnidirectional RX antenna at check point 13: (a) range plot and (b) contour plot with root raised cosine filter applied to the TX signal with an omnidirectional TX antenna. 


\section{CUP's Path Gain for 2.245 GHz: Tx Horn Vpol@ Tx2 \& Rx Omni Vpol@CP 13}

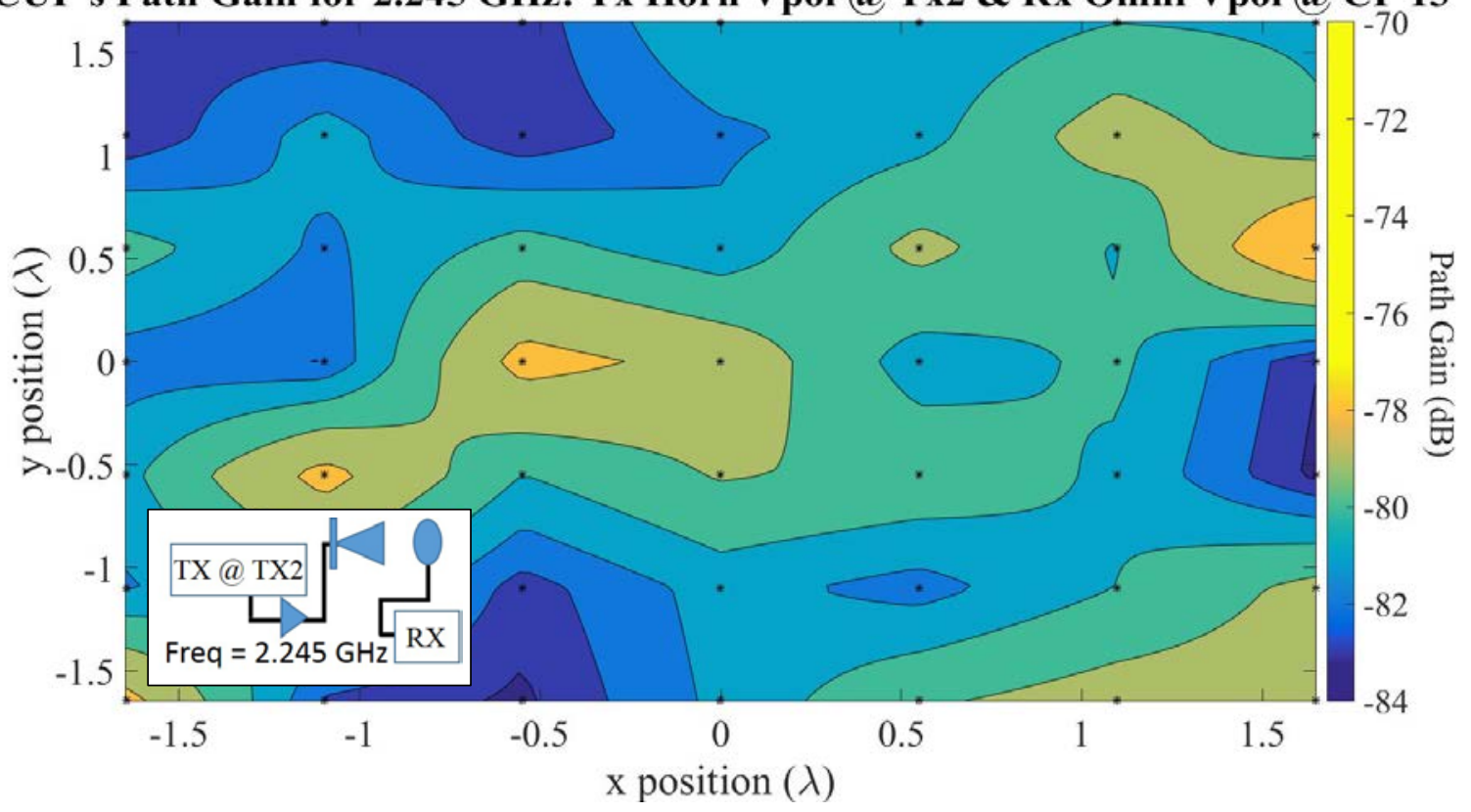

(a)

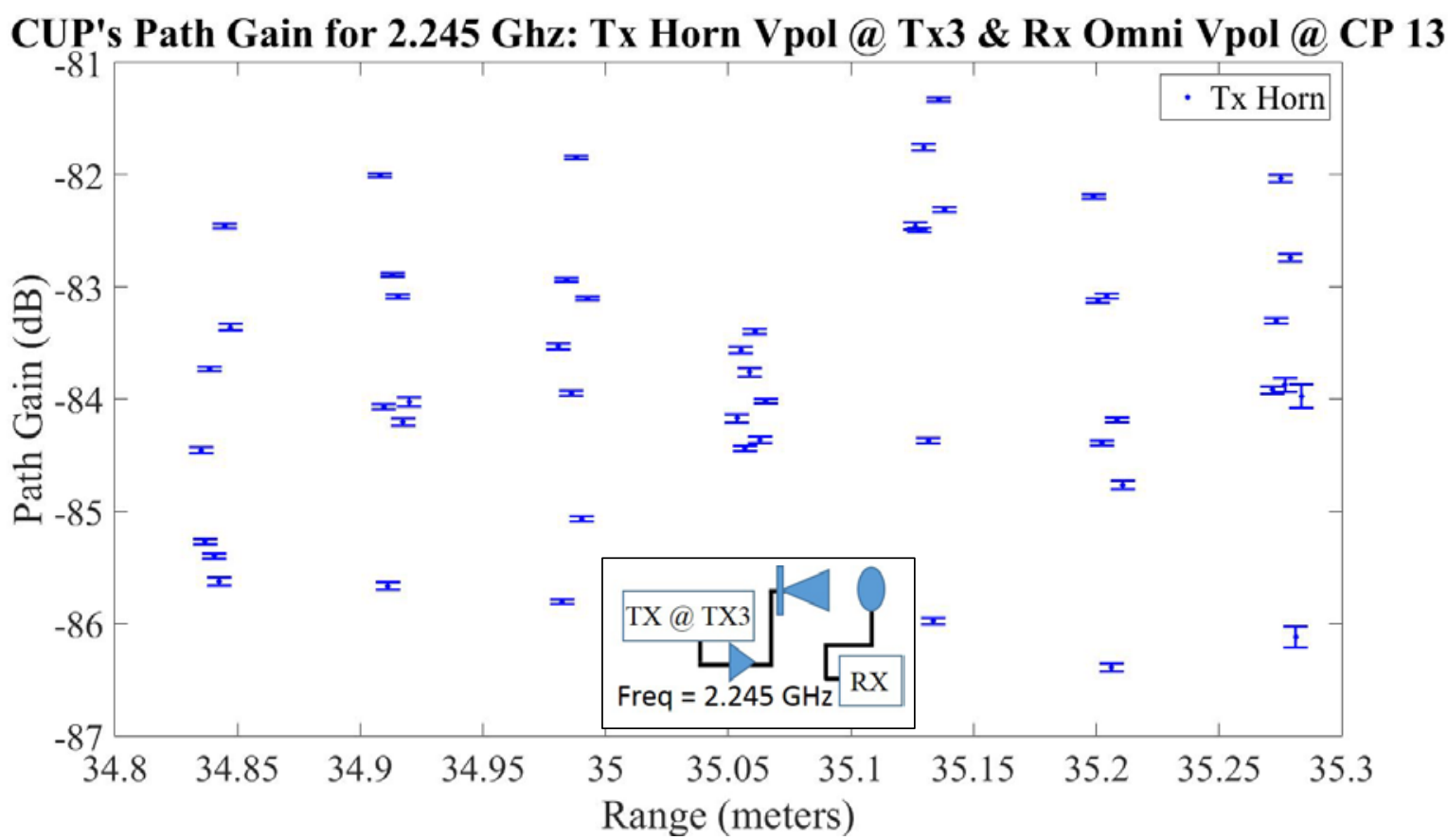

(b)

Fig. 4-14 Path gain measurements in the NIST CUP at $2.245 \mathrm{GHz}$ for Vpol horn TX antenna and Vpol omnidirectional RX antenna at check point 13: (a) contour plot at position TX2 and (b) range plot at position TX3. 


\section{CUP's Path Gain For 2.245 GHz: Tx Horn Vpol@Tx3 \& Rx Omni Vpol@CP 13}

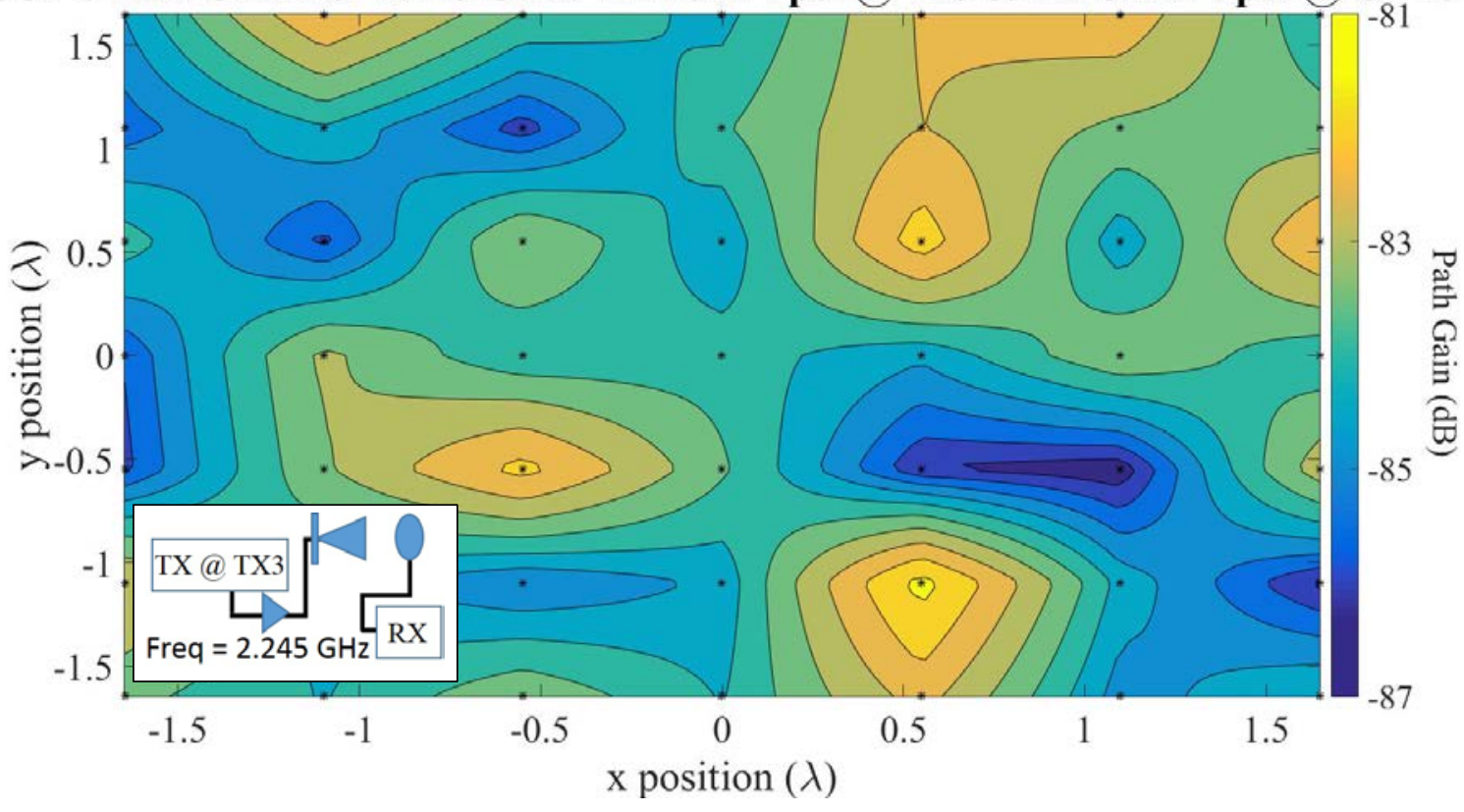

(a)

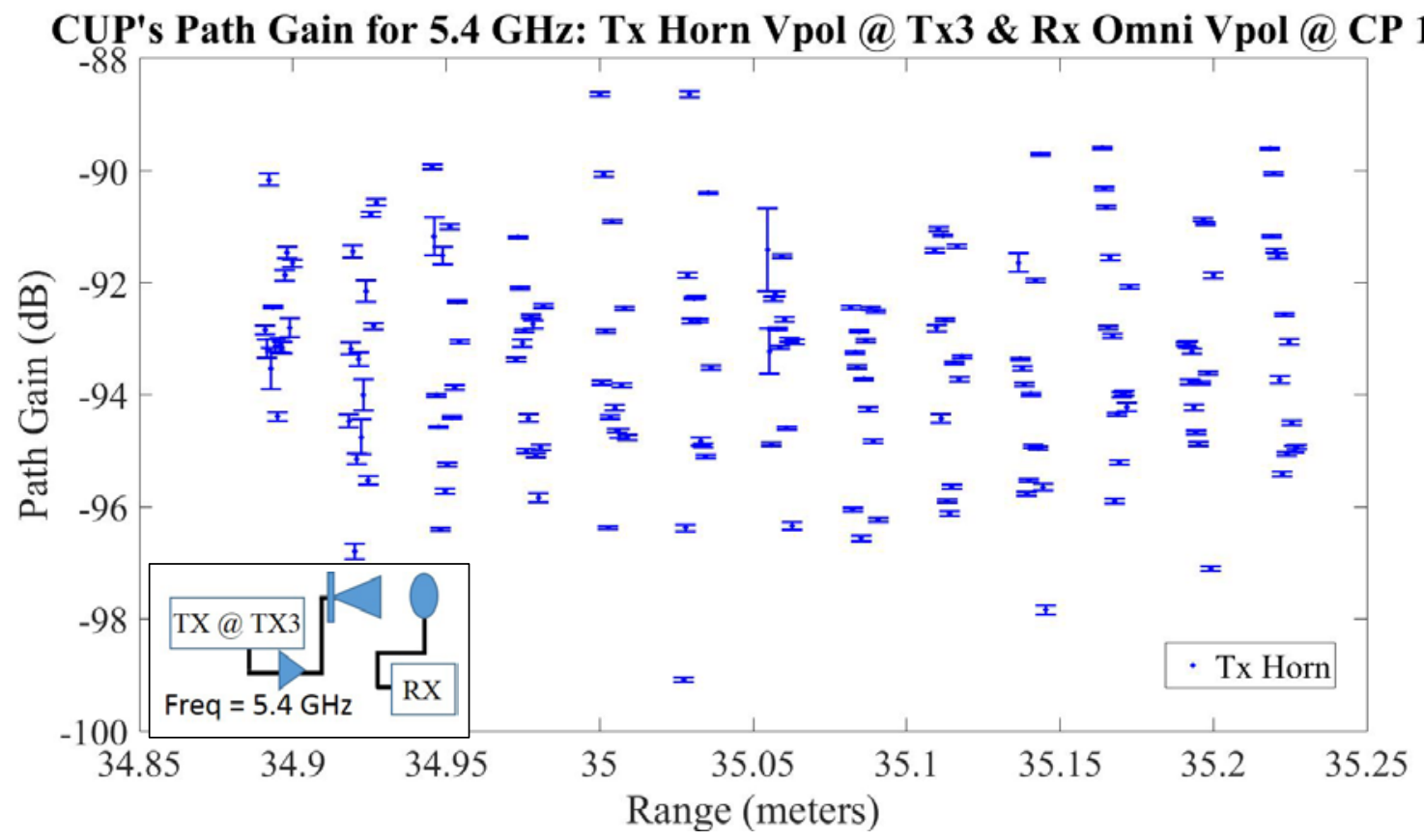

(b)

Fig. 4-15 Path gain measurements in the NIST CUP for Vpol horn TX antenna and Vpol omnidirectional RX antenna at check point 13: (a) contour plot at $2.245 \mathrm{GHz}$ and (b) range plot at $5.4 \mathrm{GHz}$. 


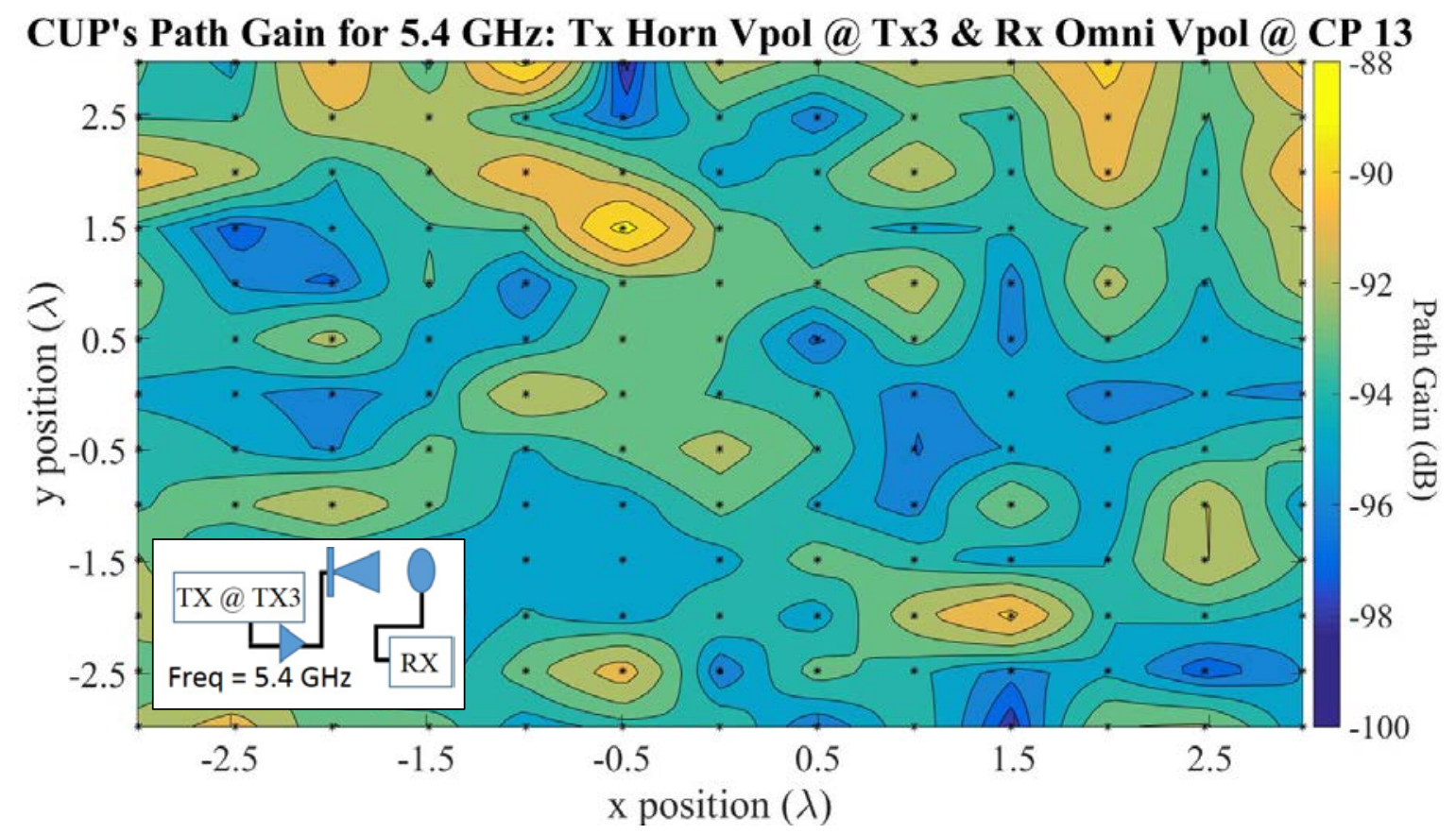

(a)

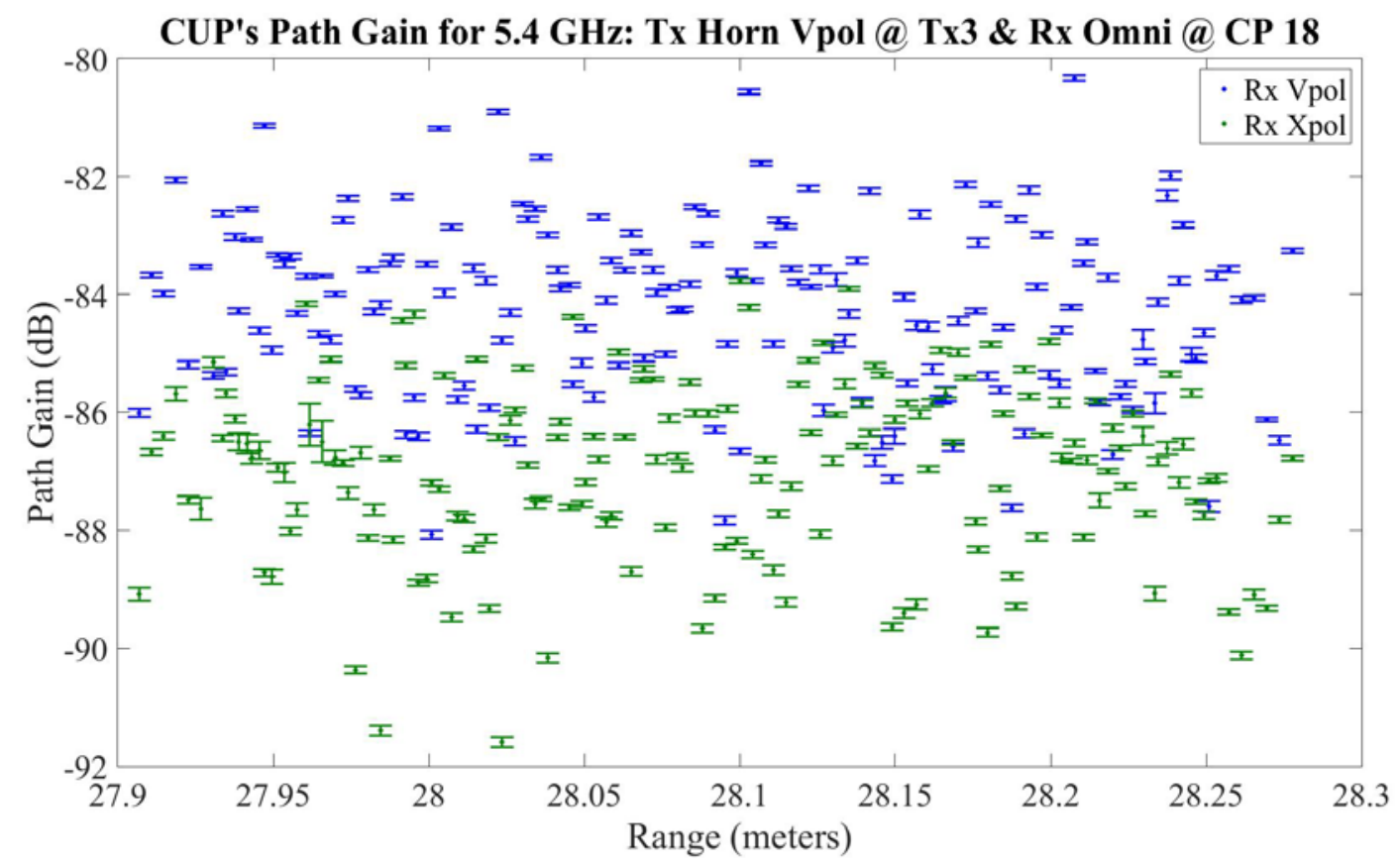

(b)

Fig. 4-16 Path gain measurements in the NIST CUP at $5.4 \mathrm{GHz}$ for Vpol horn TX antenna and omnidirectional RX antenna: (a) contour plot for Vpol Rx antenna at check point 13 and (b) range plot at $5.4 \mathrm{GHz}$ for Vpol or Xpol RX antenna at check point 18 . 
CUP's Path Gain for 5.4 GHz: Tx Horn Vpol @ Tx3 \& Rx Omni Vpol @ CP 18

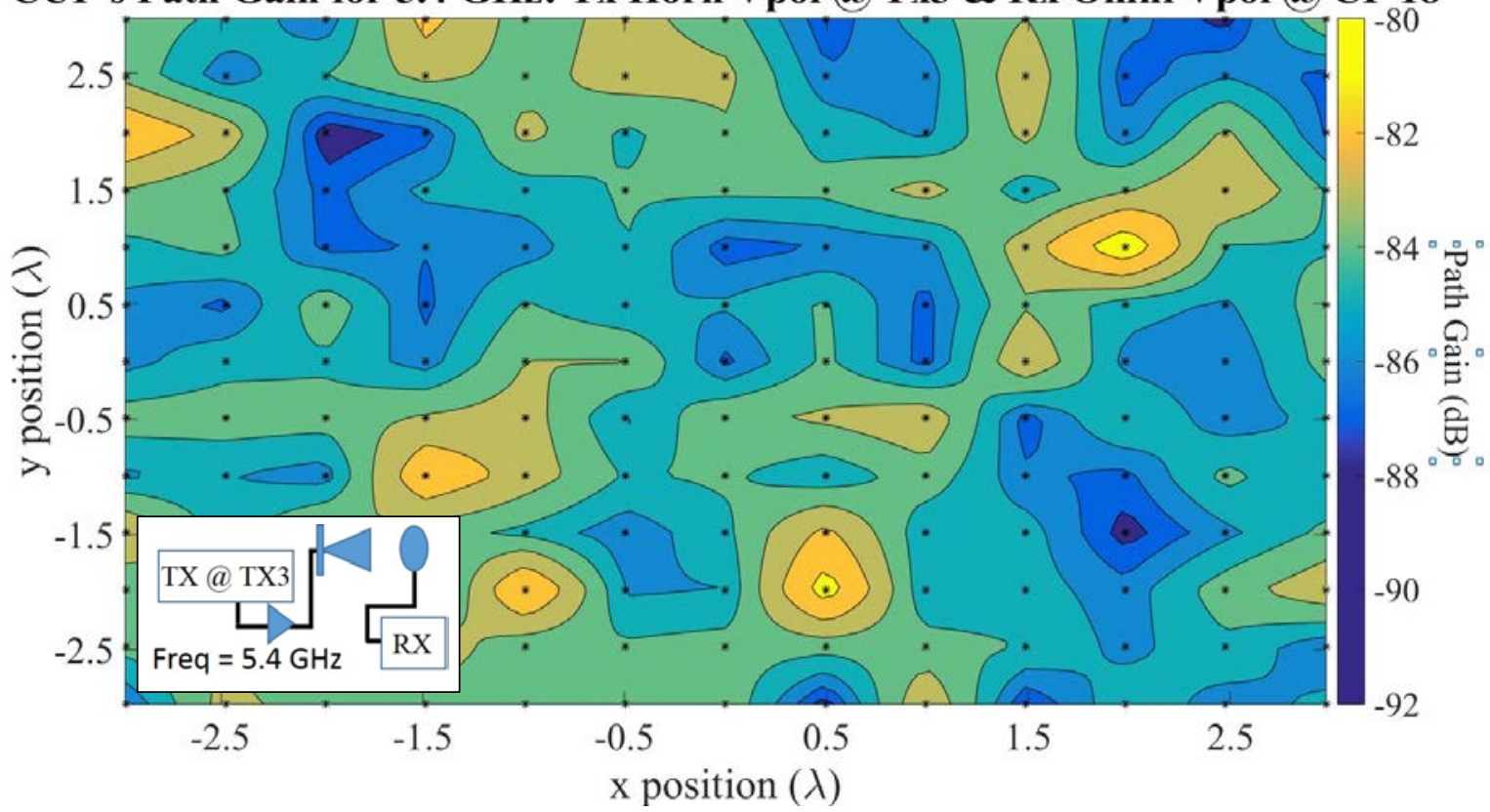

(a)

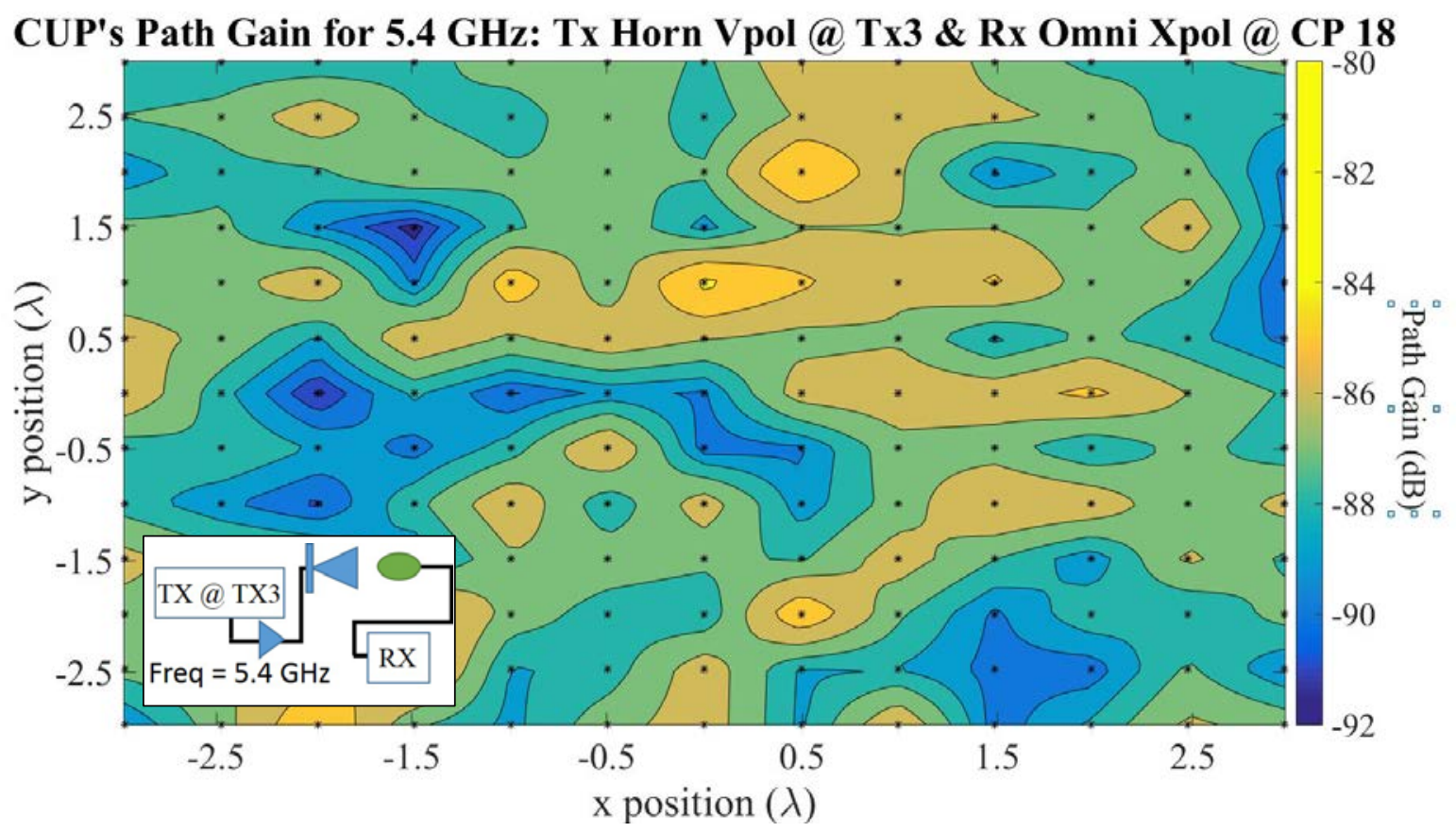

(b)

Fig. 4-17 Path gain measurements in the NIST CUP at 5.4 GHz for Vpol TX antenna at position TX3 and omnidirectional RX antenna at check point 18: (a) Vpol RX antenna and (b) Xpol RX antenna. 


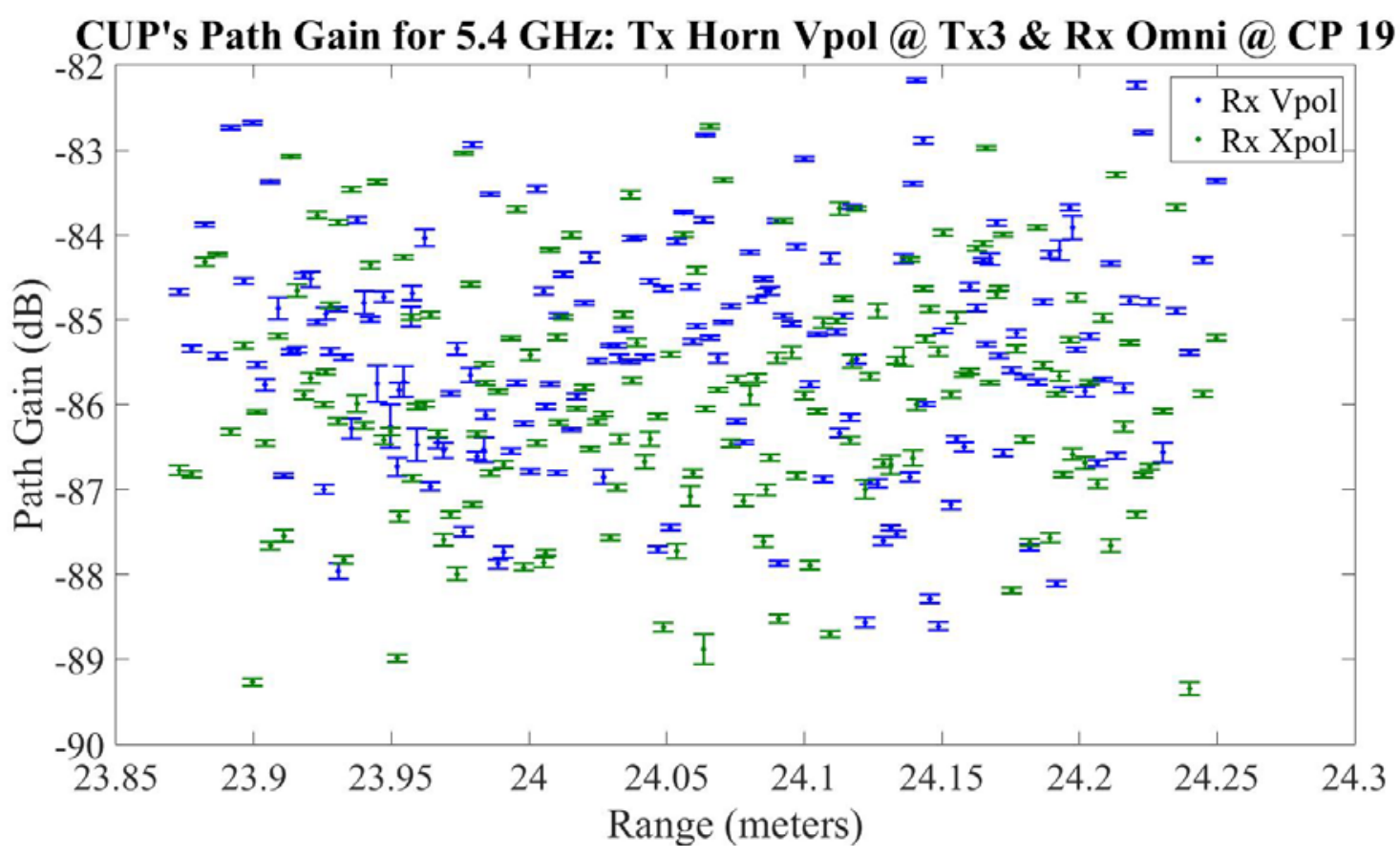

(a)

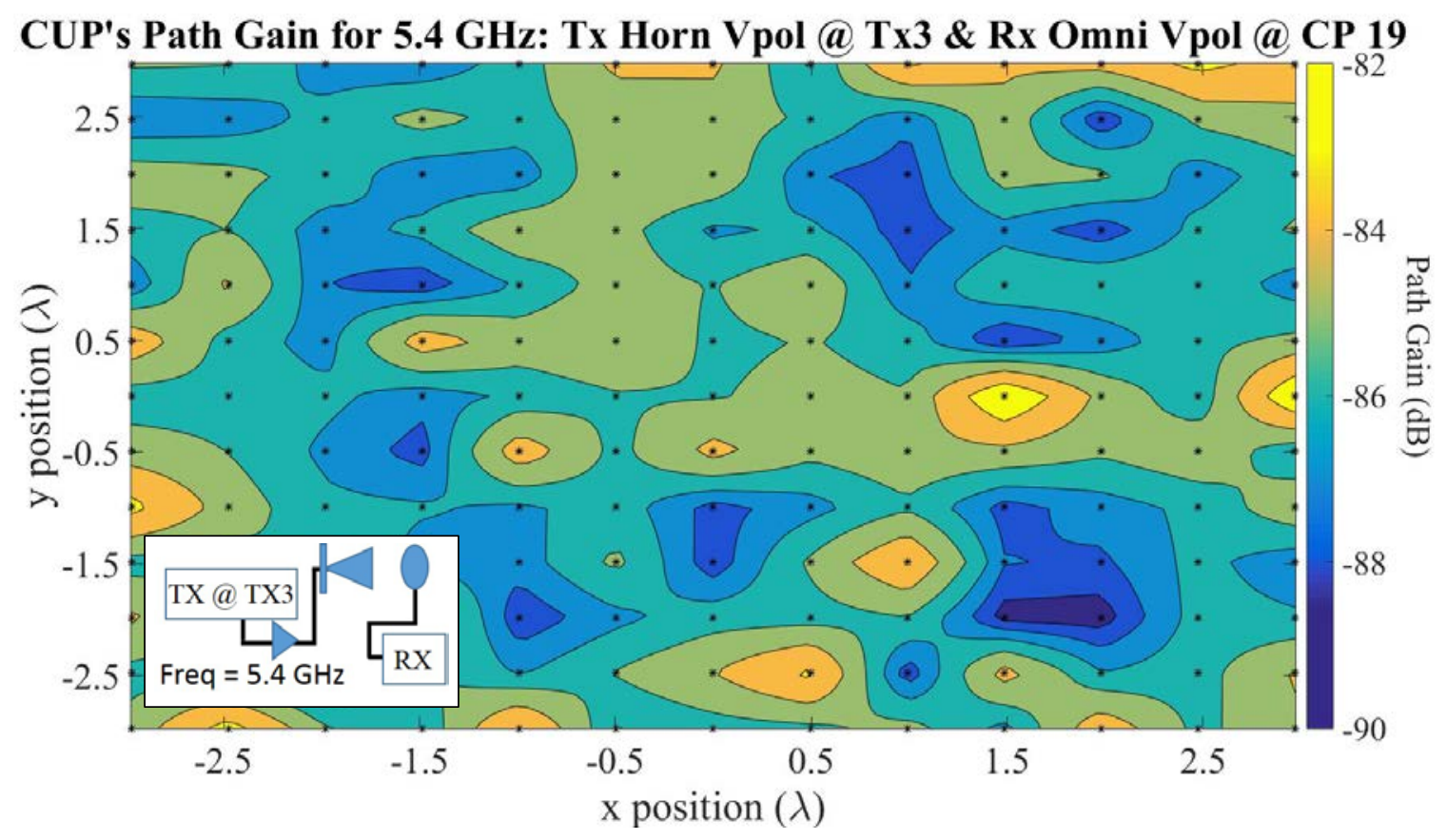

(a)

Fig. 4-18 Path gain measurements in the NIST CUP at $5.4 \mathrm{GHz}$ for Vpol horn TX antenna at position TX3 and omnidirectional RX antenna at check point 19: (a) range plot for Vpol or Xpol Rx antenna and (b) contour plot for Vpol RX antenna. 


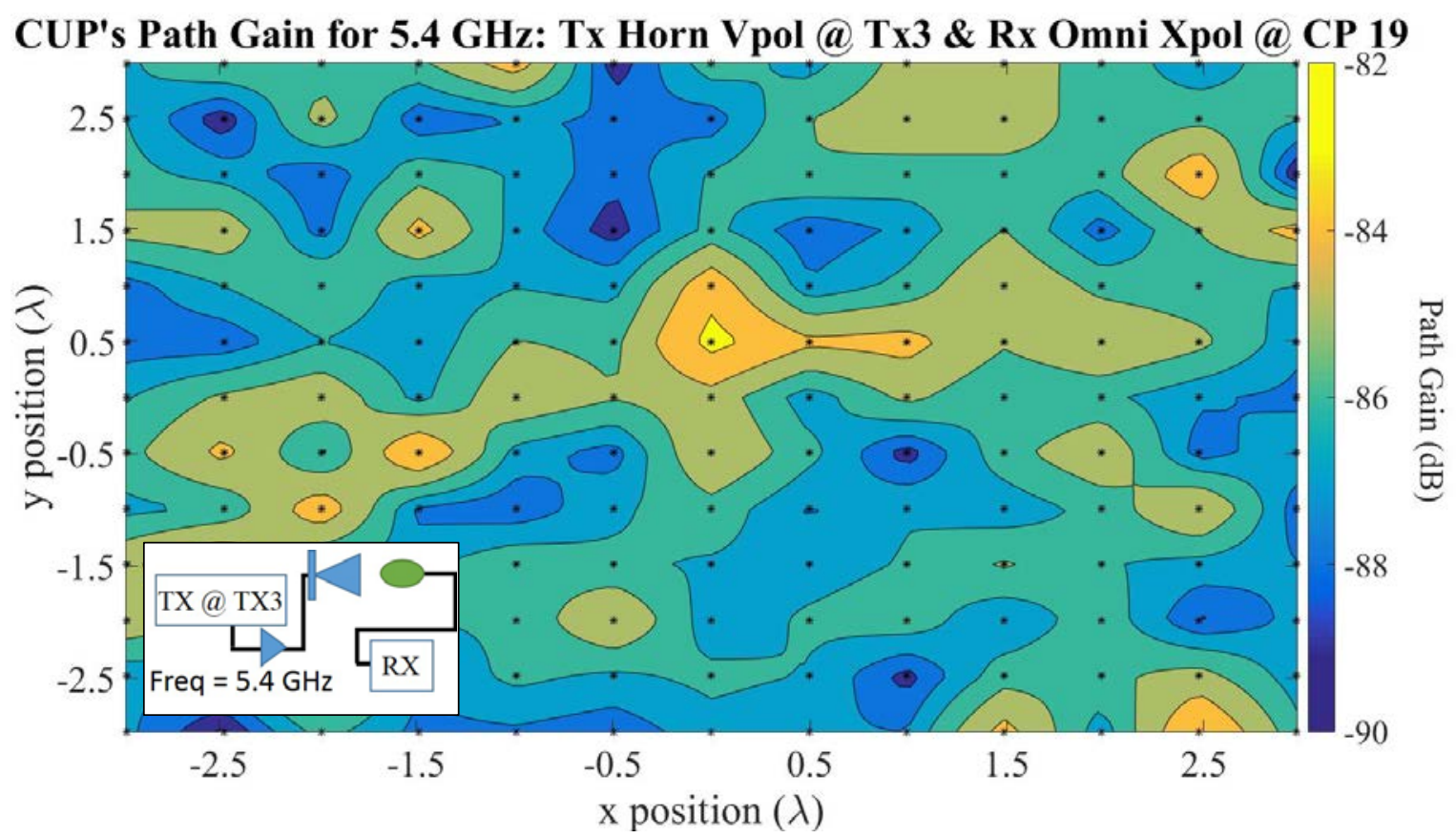

(a)

\section{CUP's Path Gain for 5.4 GHz: Tx HornVpol @ Tx3 \& Rx Omni Vpol@ CP 29}

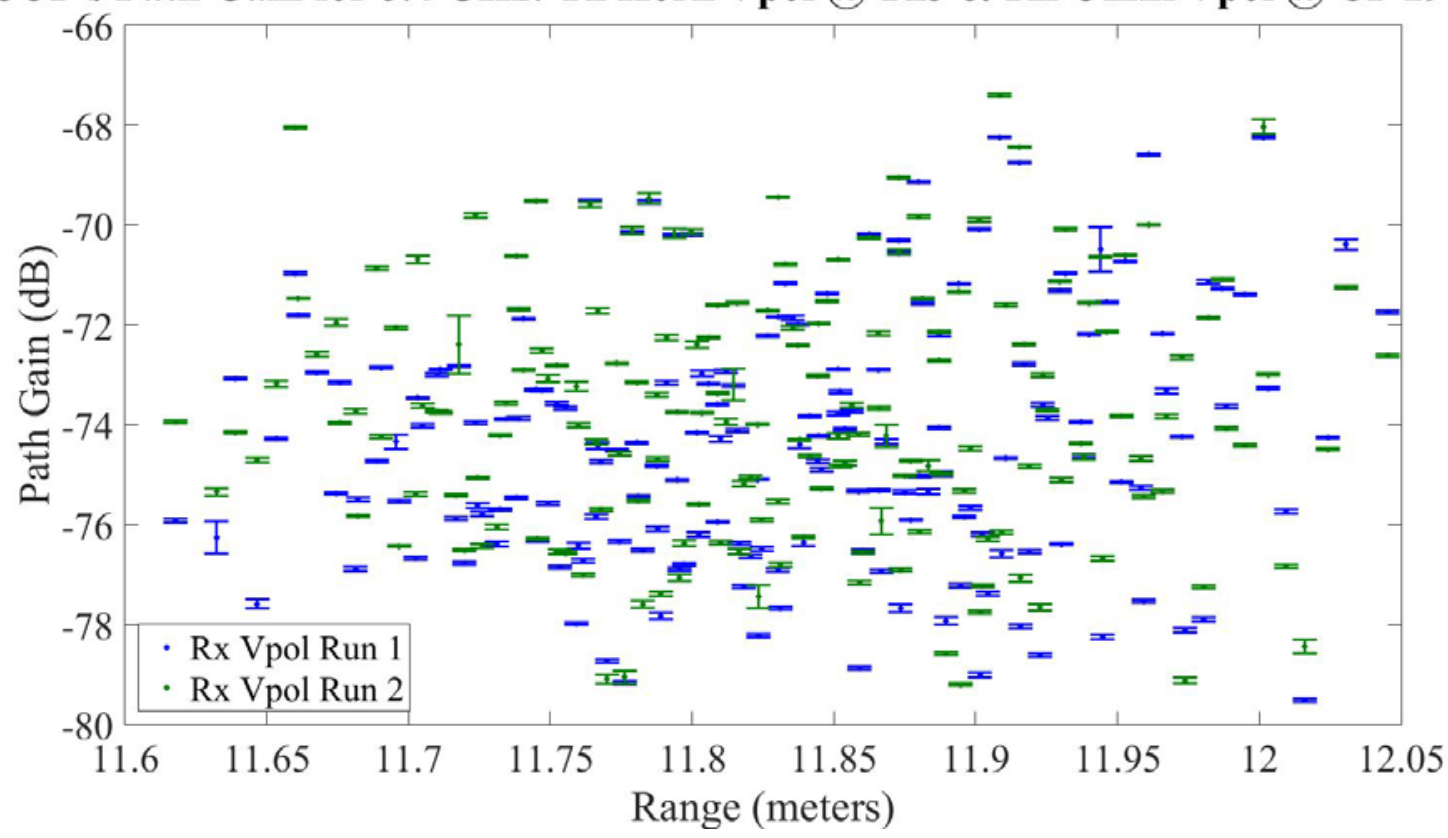

(b)

Fig. 4-19 Path gain measurements in the NIST CUP at $5.4 \mathrm{GHz}$ for Vpol horn TX antenna at position TX3 and omnidirectional RX antenna: (a) contour plot at check point 19 for Xpol RX antenna and (b) range plot at check point 29 for Vpol RX antenna. 


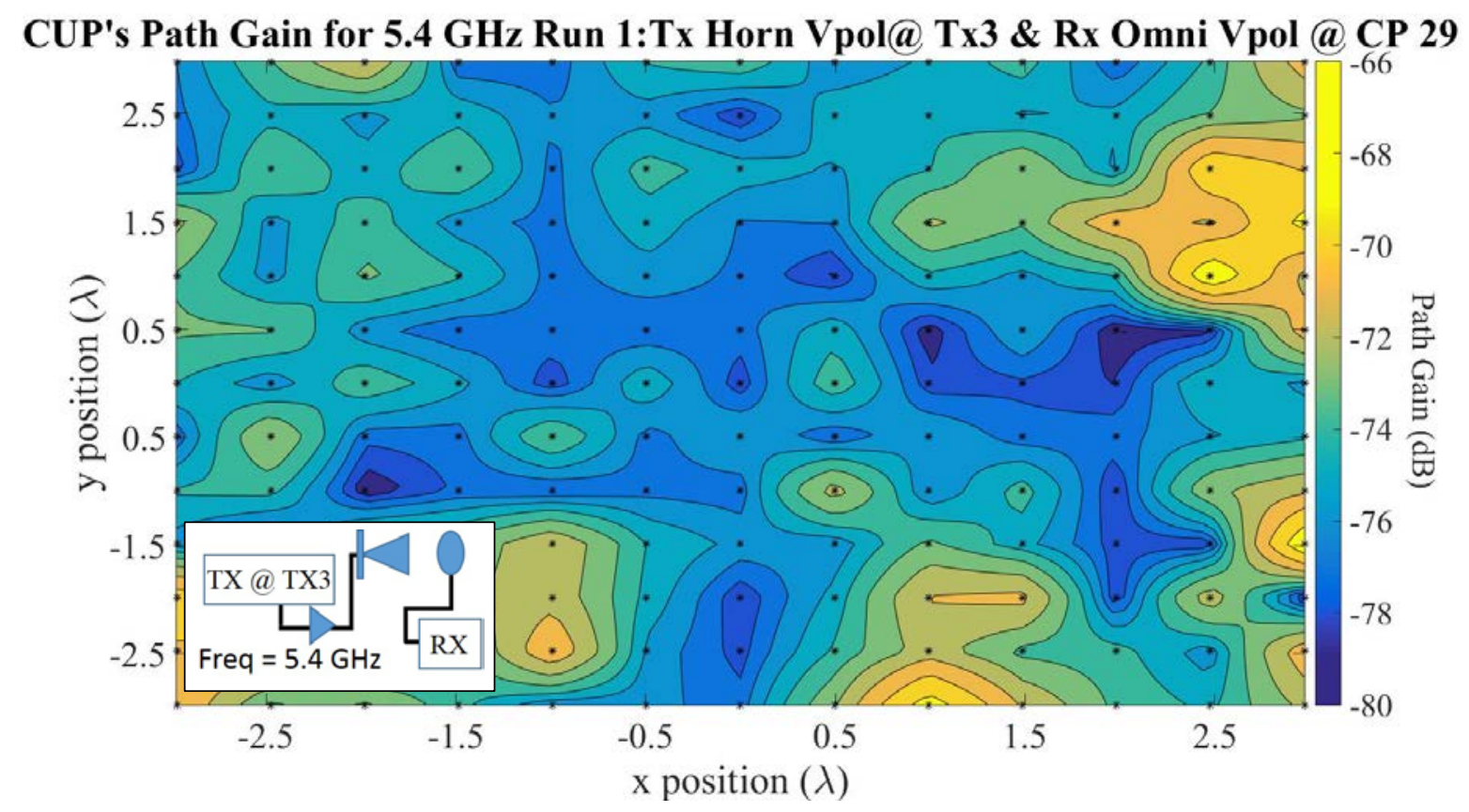

(a)

\section{CUP's Path Gain for 5.4 GHz Run 2:Tx Horn Vpol@Tx3 \& Rx Omni Vpol @ CP 29}

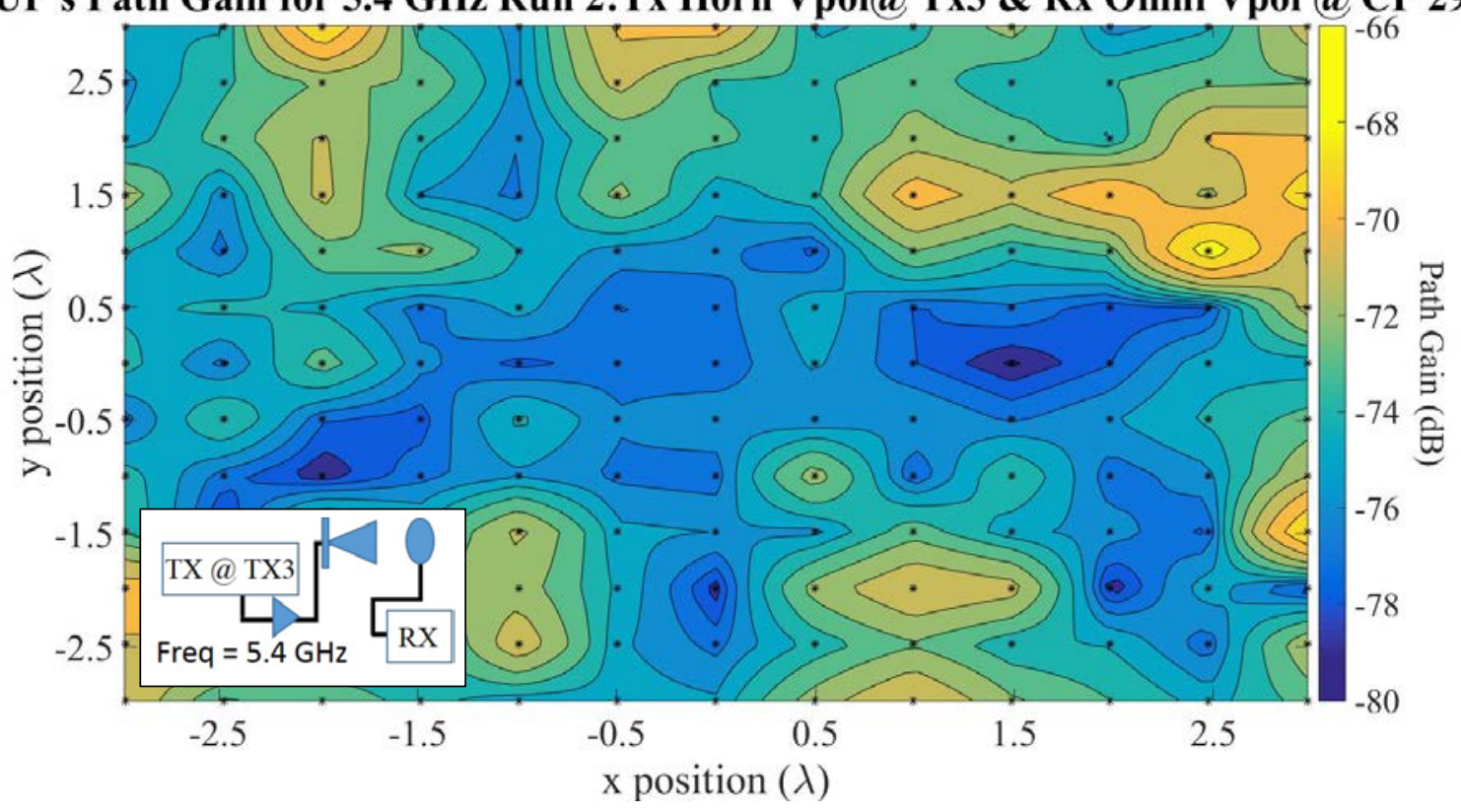

(b)

Fig. 4-20 Path gain measurements in the NIST CUP at 5.4 GHz for Vpol horn TX antenna at position TX3 and omnidirectional RX antenna at check point 29: (a) run 1 and (b) run 2 


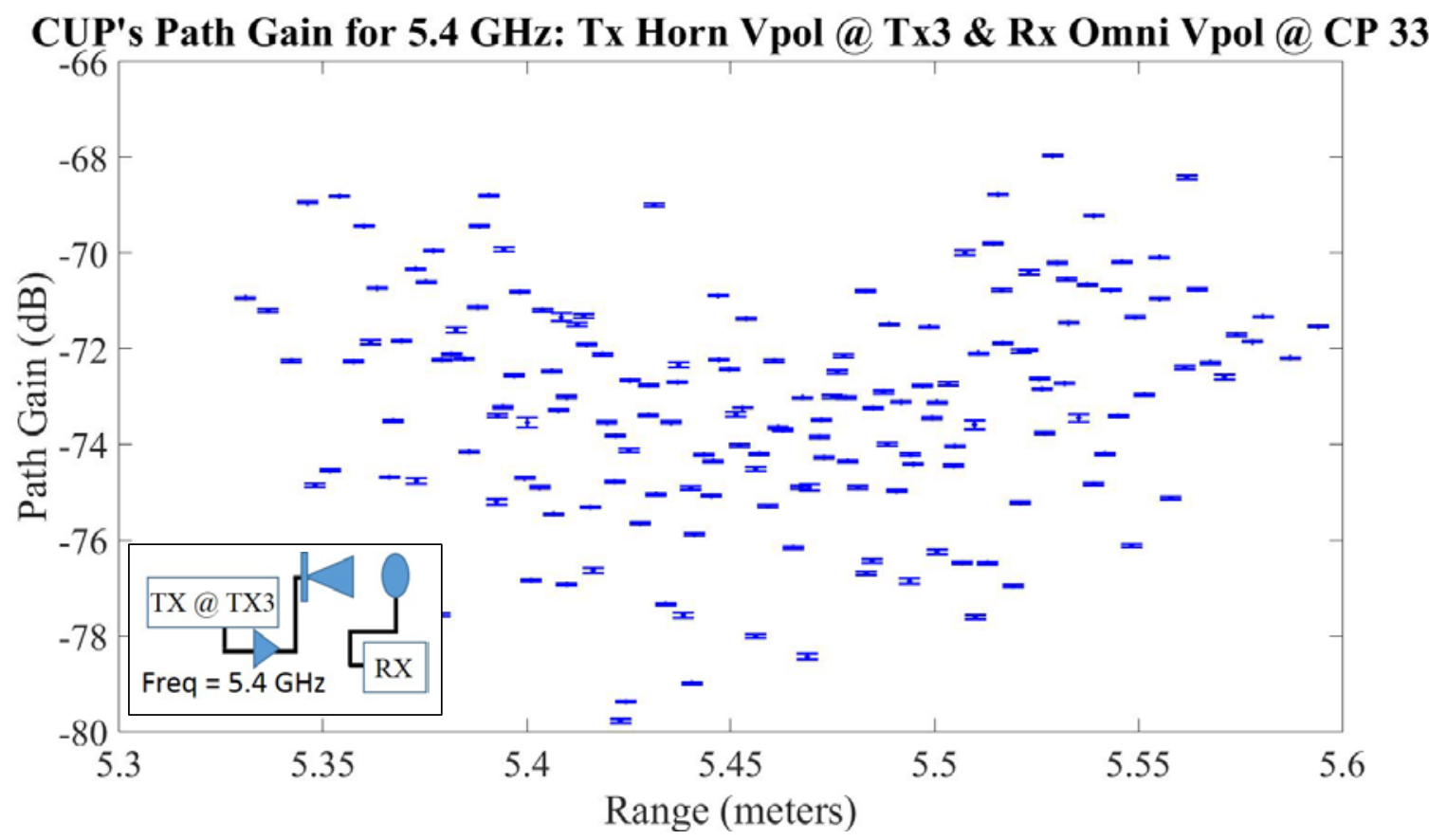

(a)

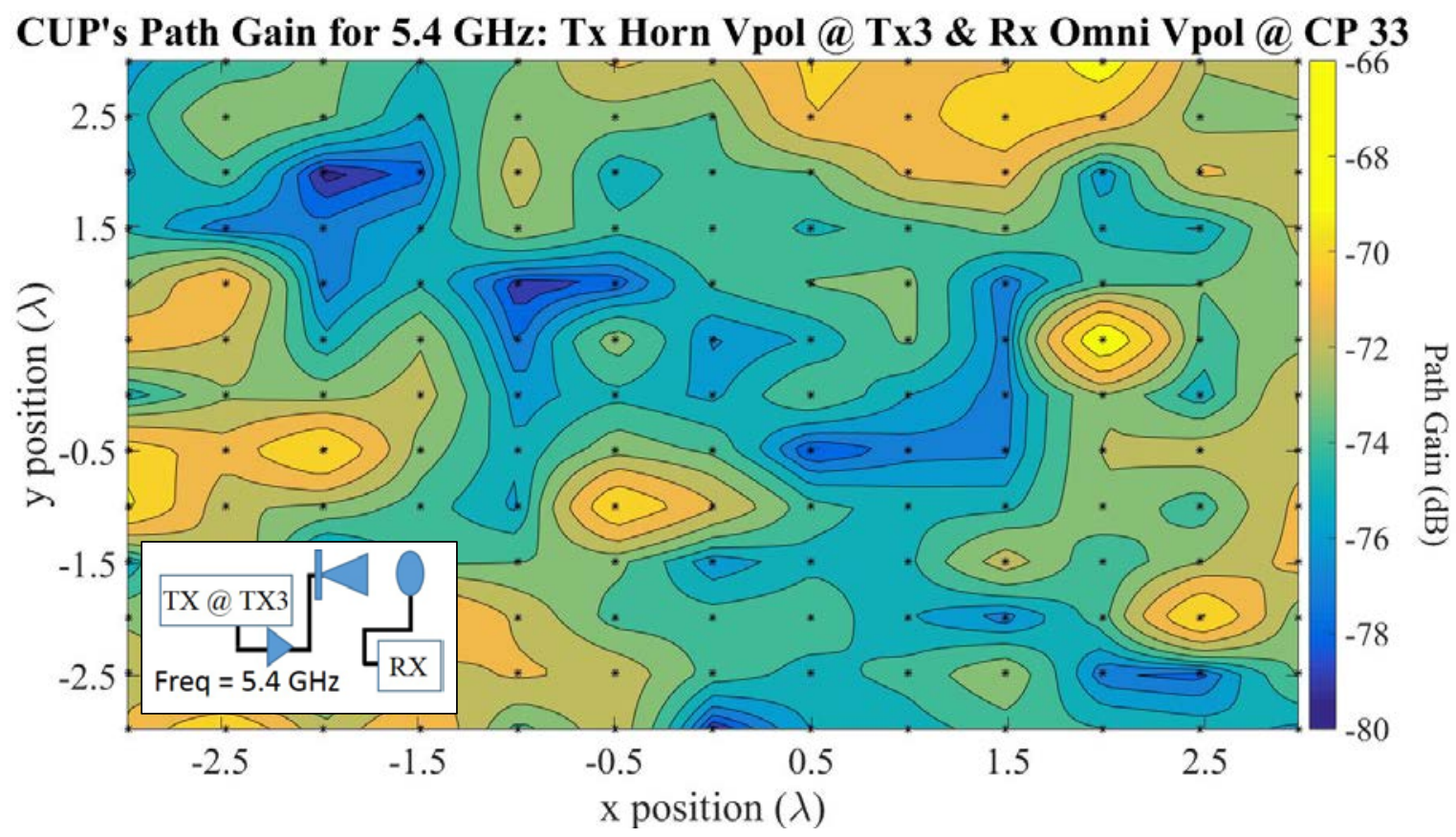

(b)

Fig. 4-21 Path gain measurements in the NIST CUP at $5.4 \mathrm{GHz}$ for Vpol horn TX antenna at position TX3 and omnidirectional RX antenna at check point 33: (a) range plot and (b) contour plot. 


\subsection{Automotive Assembly Plant (AAP) Measurement Campaign}

The Automotive Assembly Plant (AAP) is an indoor automotive assembly factory. The factory contained large tracts of open areas, machining areas, inspection machines, assembly work cells, and stacked storage areas. This factory provided a very-reflective, physically-large environment. The floor dimensions of the factory were over $400 \mathrm{~m} \mathrm{x} 400 \mathrm{~m}$ with a ceiling height of approximately $12 \mathrm{~m}$. The transmitter positions are given in Table 4-4, relative to the origin (orange circle) in Fig. 4-22.

Table 4-4: Automotive Assembly Plant TX locations.

\begin{tabular}{|c|c|c|c|}
\hline TX Location & X(m) & Y(m) & Z(m) \\
\hline TX1 & 274 & 152 & 3.1 \\
\hline TX2 & 125 & 178 & 3.1 \\
\hline
\end{tabular}

We conducted the channel sounder measurements for this campaign were conducted during the spring of 2015 using the previously described channel sounder system with a symbol rate of 20 Msym/s, $4 \frac{\text { Samples }}{\text { symbol }}$, a PN code of 2047 symbols with a maximum sequence length of 11 . The attenuation for the back-to-back measurement was $50 \mathrm{~dB}$. We used the omnidirectional TX antenna described in Section 2.1. The TX antenna was placed in one of the two locations shown in Table 4-4, during the measurement campaign at the greater heights greater than the RX antenna. The RX antenna height was $1.92 \mathrm{~m}$. The RX antenna was the omnidirectional dipole described in Section 2.1. The post processing of the measurement data used a PNideal filter with discrete sum scaling.

A number of measurement runs were conducted during the AAP measurement campaign and the measurement data may be downloaded from the website, http://doi.org/10.18434/T44S3N. 
Table 4-5: AAP runs: omnidirectional = biconical antenna .

\begin{tabular}{|c|c|c|c|c|c|c|}
\hline $\begin{array}{c}\text { Channel } \\
\text { Sounder } \\
\text { Information }\end{array}$ & $\begin{array}{l}\text { Freq. } \\
\text { (GHz) }\end{array}$ & $\begin{array}{c}\text { TX } \\
\text { Position } \\
\text { and } \\
\text { Route }\end{array}$ & $\begin{array}{c}\text { Path Gain Model } \\
\text { B, A(dB) }\end{array}$ & $\begin{array}{l}\text { Acquisitio } \\
\text { n Numbers } \\
\text { (start,end) }\end{array}$ & Figure \# & $\begin{array}{l}\text { Measurement } \\
\text { Filename }\end{array}$ \\
\hline $\begin{array}{l}\text { RX: Vertical Pol. } \\
\text { TX: Vertical Pol. } \\
\text { Omnidirectional }\end{array}$ & 2.245 & TX1, O & $\begin{array}{c}r_{B P}=54 \\
\mathrm{~B} 1=-1.1, \mathrm{~A} 1=-50 \\
\mathrm{~B} 2=-4.2, \mathrm{~A} 2=-6.4\end{array}$ & 22,142 & Fig. 4-23(a) & $\begin{array}{l}\text { AAPlantD1_2GHz } \\
\text { _TX1_vpol_run3 }\end{array}$ \\
\hline $\begin{array}{l}\text { RX: Horizontal Pol. } \\
\text { TX: Vertical Pol. } \\
\text { Omnidirectional }\end{array}$ & 2.245 & TX1, O & $\begin{array}{c}r_{B P}=54 \\
\mathrm{~B} 1=-1.2, \mathrm{~A} 1=-54 \\
\mathrm{~B} 2=-5.2, \mathrm{~A} 2=9\end{array}$ & 12,132 & Fig. 4-23(a) & $\begin{array}{l}\text { AAPlantD1_2GHz } \\
\text { _TX1_hpol_run4 }\end{array}$ \\
\hline $\begin{array}{l}\text { RX: Vertical Pol. } \\
\text { TX: Vertical Pol. } \\
\text { Omnidirectional }\end{array}$ & 5.4 & TX1, O & $\begin{array}{c}r_{B P}=54 \\
\mathrm{~B} 1=-0.9, \mathrm{~A} 1=-58 \\
\mathrm{~B} 2=-3.8, \mathrm{~A} 2=-16\end{array}$ & 11,118 & Fig. 4-23(b) & $\begin{array}{l}\text { AAPlantD2_5GHz } \\
\text { _TX1_vpol_run1 }\end{array}$ \\
\hline $\begin{array}{l}\text { RX: Horizontal Pol. } \\
\text { TX: Vertical Pol. } \\
\text { Omnidirectional }\end{array}$ & 5.4 & TX1, O & $\begin{array}{c}r_{B P}=54 \\
\mathrm{~B} 1=-0.9, \mathrm{~A} 1=-64 \\
\mathrm{~B} 2=-4.3, \mathrm{~A} 2=-12\end{array}$ & 4,113 & Fig. 4-23(b) & $\begin{array}{l}\text { AAPlantD2_5GHz } \\
\text { _TX1_hpol_run2 }\end{array}$ \\
\hline $\begin{array}{l}\text { RX: Vertical Pol. } \\
\text { TX: Vertical Pol. } \\
\text { Omnidirectional }\end{array}$ & 2.245 & TX1, I & $\begin{array}{c}r_{B P}=20 \\
\mathrm{~B} 1=-0.37, \mathrm{~A} 1=-56 \\
\mathrm{~B} 2=-4.7, \mathrm{~A} 2=2.8\end{array}$ & 6,101 & Fig. 4-24(a) & $\begin{array}{l}\text { AAPlantD2_2GHz } \\
\text { _TX1_vpol_intern } \\
\text { al_runF }\end{array}$ \\
\hline $\begin{array}{l}\text { RX: Horizontal Pol. } \\
\text { TX: Vertical Pol. } \\
\text { Omnidirectional }\end{array}$ & 2.245 & TX1, I & $\begin{array}{c}r_{B P}=24 \\
\mathrm{~B} 1=-0.57, \mathrm{~A} 1=-58 \\
\mathrm{~B} 2=-5.0, \mathrm{~A} 2=5.2\end{array}$ & 5,104 & Fig. 4-24(a) & $\begin{array}{l}\text { AAPlantD2_2GHz } \\
\text { _TX1_hpol_intern } \\
\text { al_runE }\end{array}$ \\
\hline $\begin{array}{l}\text { RX: Vertical Pol. } \\
\text { TX: Vertical Pol. } \\
\text { Omnidirectional } \\
\end{array}$ & 5.4 & TX1, I & $\begin{array}{c}r_{B P}=24 \\
\mathrm{~B} 1=-0.4, \mathrm{~A} 1=-62 \\
\mathrm{~B} 2=-4.3, \mathrm{~A} 2=-8.3\end{array}$ & 35,127 & Fig. 4-24(b) & $\begin{array}{l}\text { AAPlantD2_5GHz } \\
\text { _TX1_vpol_intern } \\
\text { al_runA }\end{array}$ \\
\hline $\begin{array}{l}\text { RX: Horizontal Pol. } \\
\text { TX: Vertical Pol. } \\
\text { Omnidirectional }\end{array}$ & 5.4 & TX1, I & $\begin{array}{c}r_{B P}=43 \\
\mathrm{~B} 1=-1.4, \mathrm{~A} 1=-52 \\
\mathrm{~B} 2=-4.9, \mathrm{~A} 2=5.3\end{array}$ & 6,94 & Fig. 4-24(b) & $\begin{array}{l}\text { AAPlantD2_5GHz } \\
\text { _TX1_hpol_intern } \\
\text { al_runB }\end{array}$ \\
\hline $\begin{array}{l}\text { RX: Vertical Pol. } \\
\text { TX: Vertical Pol. } \\
\text { Omnidirectional }\end{array}$ & 2.245 & TX2, O & $\begin{array}{c}r_{B P}=272 \\
\mathrm{~B} 1=-3.9, \mathrm{~A} 1=-11 \\
\mathrm{~B} 2=0.0, \mathrm{~A} 2=0.0\end{array}$ & 13,133 & Fig. 4-25(a) & $\begin{array}{l}\text { AAPlantD3_2GHz } \\
\text { _TX2_vpol_run32 }\end{array}$ \\
\hline $\begin{array}{l}\text { RX: Horizontal Pol. } \\
\text { TX: Vertical Pol. } \\
\text { Omnidirectional }\end{array}$ & 2.245 & TX2, O & $\begin{array}{c}r_{B P}=272 \\
\mathrm{~B} 1=-4.0, \mathrm{~A} 1=-13 \\
\mathrm{~B} 2=0.0, \mathrm{~A} 2=0.0\end{array}$ & 7,113 & Fig. 4-25(a) & $\begin{array}{l}\text { AAPlantD3_2GHz } \\
\text { _TX2_hpol_run34 }\end{array}$ \\
\hline $\begin{array}{l}\text { RX: Vertical Pol. } \\
\text { TX: Vertical Pol. } \\
\text { Omnidirectional }\end{array}$ & 5.4 & TX2, O & $\begin{array}{c}r_{B P}=272 \\
\mathrm{~B} 1=-3.3, \mathrm{~A} 1=-26 \\
\mathrm{~B} 2=0.0, \mathrm{~A} 2=0.0\end{array}$ & 20,136 & Fig. 4-25(b) & $\begin{array}{l}\text { AAPlantD3_5GHz } \\
\text { _TX2_vpol_run37 }\end{array}$ \\
\hline $\begin{array}{l}\text { RX: Horizontal Pol. } \\
\text { TX: Vertical Pol. } \\
\text { Omnidirectional } \\
\end{array}$ & 5.4 & TX2, O & $\begin{array}{c}r_{B P}=272 \\
\mathrm{~B} 1=-3.4, \mathrm{~A} 1=-29 \\
\mathrm{~B} 2=0.0, \mathrm{~A} 2=0.0\end{array}$ & 18,117 & Fig. 4-25(b) & $\begin{array}{l}\text { AAPlantD3_5GHz } \\
\text { _TX2_hpol_run38 }\end{array}$ \\
\hline $\begin{array}{l}\text { RX: Vertical Pol. } \\
\text { TX: Vertical Pol. } \\
\text { Omnidirectional }\end{array}$ & 2.245 & TX2, I & $\begin{array}{c}r_{B P}=150 \\
\mathrm{~B} 1=-4.9, \mathrm{~A} 1=6.2 \\
\mathrm{~B} 2=0.0, \mathrm{~A} 2=0.0\end{array}$ & 49,143 & Fig. 4-26(a) & $\begin{array}{l}\text { AAPlantD3_2GHz } \\
\text { _TX2_vpol_intern } \\
\text { al_run33 }\end{array}$ \\
\hline $\begin{array}{l}\text { RX: Horizontal Pol. } \\
\text { TX: Vertical Pol. } \\
\text { Omnidirectional }\end{array}$ & 2.245 & TX2, I & $\begin{array}{c}r_{B P}=150 \\
\mathrm{~B} 1=-5.0, \mathrm{~A} 1=4.9 \\
\mathrm{~B} 2=0.0, \mathrm{~A} 2=0.0\end{array}$ & 11,103 & Fig. 4-26(a) & $\begin{array}{l}\text { AAPlantD3_2GHz } \\
\text { _TX2_hpol_intern } \\
\text { al_run36 }\end{array}$ \\
\hline $\begin{array}{l}\text { RX: Horizontal Pol. } \\
\text { TX: Vertical Pol. } \\
\text { Omnidirectional }\end{array}$ & 5.4 & TX2, I & $\begin{array}{c}r_{B P}=150 \\
\mathrm{~B} 1=-4.8, \mathrm{~A} 1=-3.5 \\
\mathrm{~B} 2=0.0, \mathrm{~A} 2=0.0\end{array}$ & 15,99 & Fig. 4-26(b) & $\begin{array}{l}\text { AAPlantD3_5GHz } \\
\text { _TX2_hpol_intern } \\
\text { al_run39 }\end{array}$ \\
\hline $\begin{array}{l}\text { RX: Vertical Pol. } \\
\text { TX: Vertical Pol. } \\
\text { Omnidirectional }\end{array}$ & 5.4 & TX2, I & $\begin{array}{c}r_{B P}=150 \\
\mathrm{~B} 1=-4.4, \mathrm{~A} 1=-5.7 \\
\mathrm{~B} 2=0.0, \mathrm{~A} 2=0.0\end{array}$ & 9,103 & Fig. 4-26(b) & $\begin{array}{l}\text { AAPlantD3_5GHz } \\
\text { _TX2_vpol_intern } \\
\text { al_run40 }\end{array}$ \\
\hline
\end{tabular}




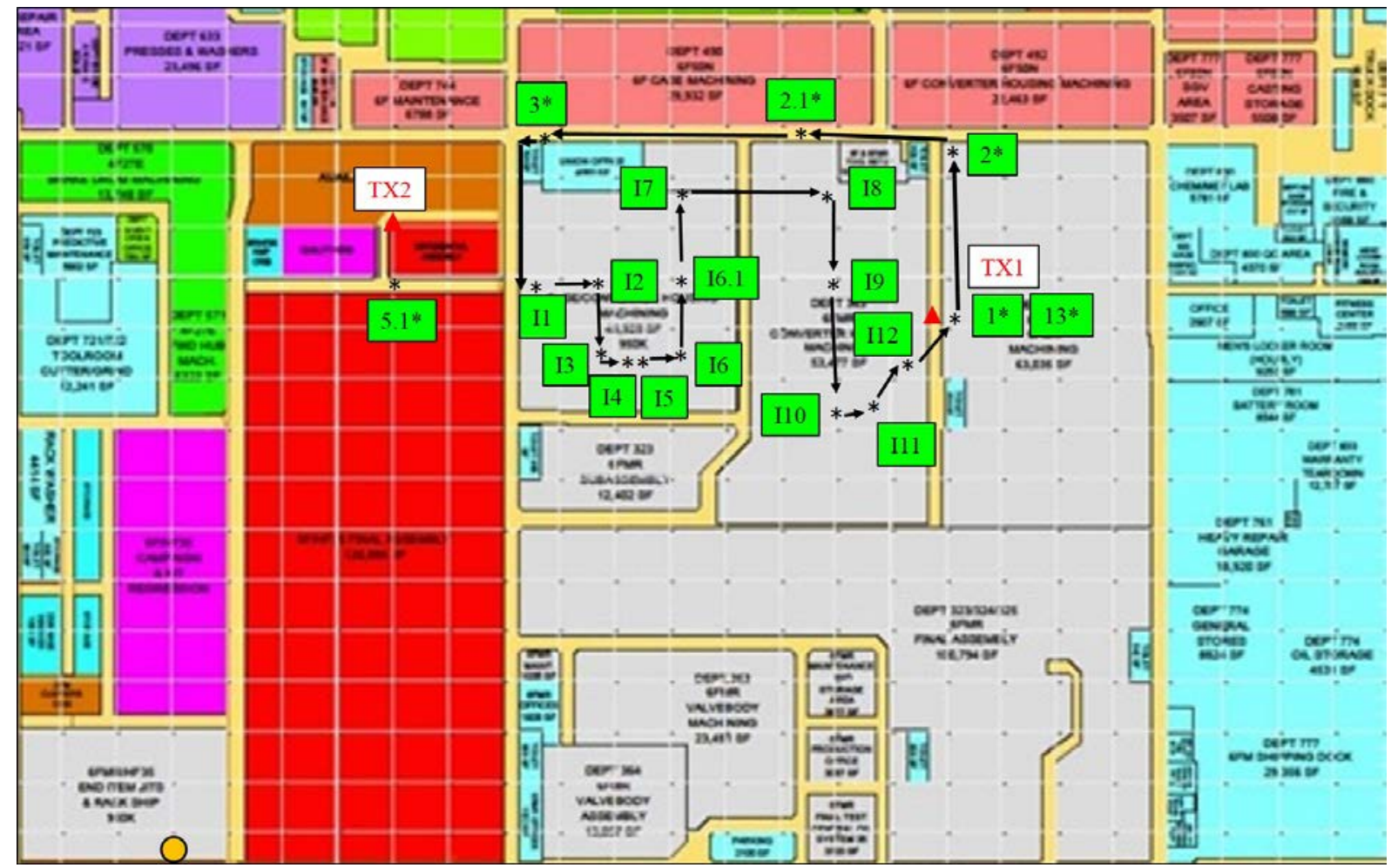

(a)

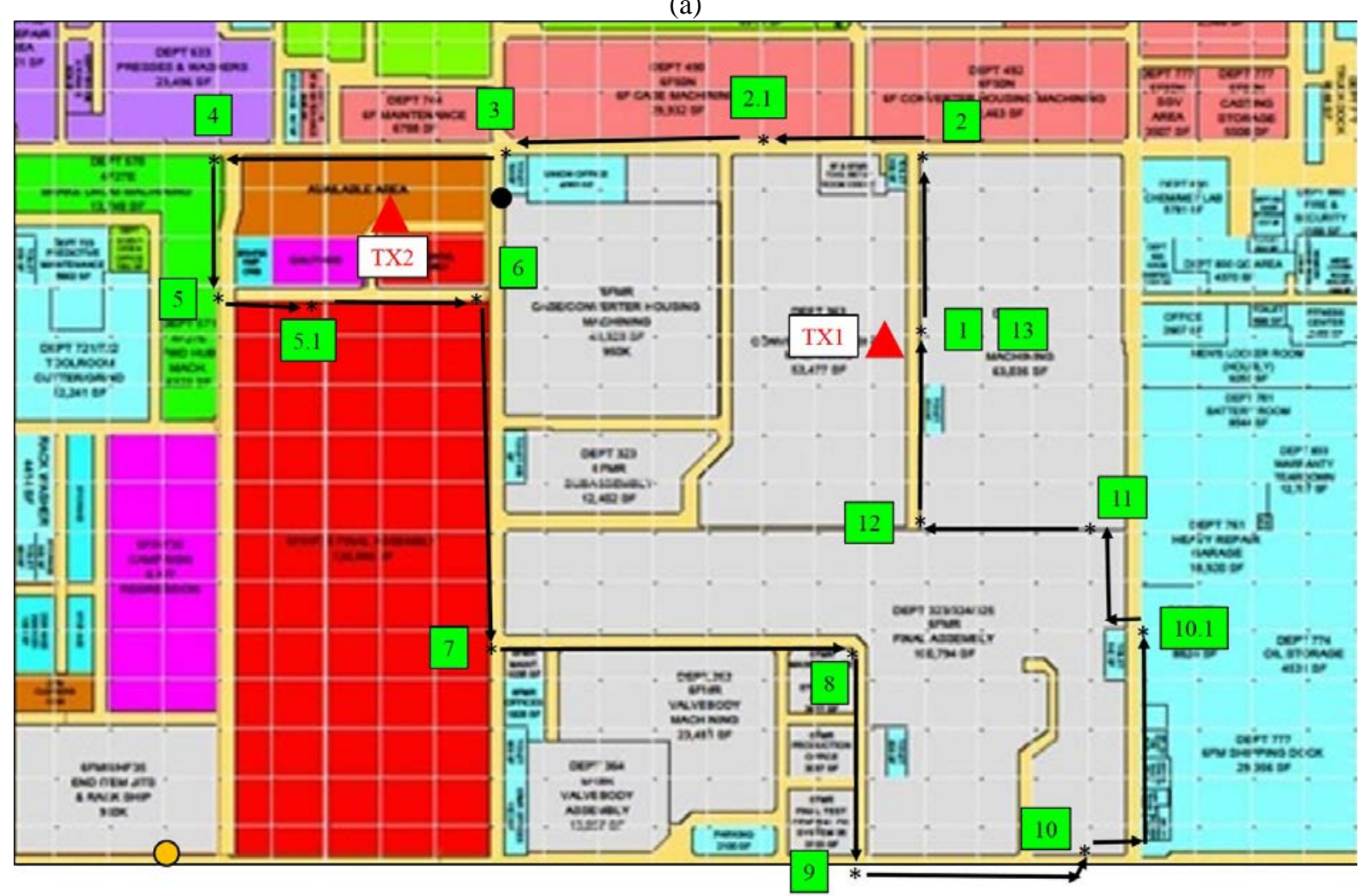

(b)

Fig. 4-22 Measurement routes through the automotive assembly plant facility: (a) inner loop and (b) outer loop. 
The AAP had an inner loop route for the measurement campaign that was conducted over 3 days. Fig. 4-22(a) shows the inner loop route with TX antenna locations and check points. The inner loop route had reflective metals, machine canyons, and overhead robot gantry systems. This route was comprised of different multipaths from LOS to highly multipath NLOS almost to the level of complete obstruction. The maximum range for this loop was approximately $125 \mathrm{~m}$. Fig. 4-15 and Fig. 4-17 show the path gain for the inner loop route compared to free space. Using a two-slope polynomial fit, we performed a linear regression on the path gain results with a break point at 15 $\mathrm{m}$ for all measurement runs. The slope of the linear regression fit can be found in Table 4-5. At ranges less than $15 \mathrm{~m}$, the slopes are low, usually below $-0.5 \mathrm{~dB} / \mathrm{m}$, (please see Section 4.1 ). After $15 \mathrm{~m}$, the slopes range from -3.3 to $-4.0 \mathrm{~dB} / \mathrm{m}$ for vertically polarized $\mathrm{RX}$ antennas. For a horizontally polarized RX antenna, the slopes have a greater range from -2.2 to $-4.5 \mathrm{~dB} / \mathrm{m}$.

The internal loop route measurement runs: designated run E, run F, run A, and run B, started at check point $1 *$, near the TX1 as shown in Fig. 4-22(a). The route took the cart away from TX1 and moved towards check point $2^{*}$ and slowly came back to check point $1^{*}$ near TX1. This route differs from the runs designated run 33 , run 36 , run 39 , and run 40 . For those runs, the cart started at check point $1 *$ far away from the transmitting antenna TX2. The cart slowly moved towards TX2, then away, finally ending at check point $1^{*}$. With the same break point at $15 \mathrm{~m}$, the slopes between these two different internal routes moving towards or away from the transmitting locations are very comparable.

The AAP also had an outer loop route used for the measurement campaign. Fig. 4-22(b) shows the outer loop route with TX antenna locations and check points. The outer loop route represents the routes that may be taken by autonomous ground vehicles (AGVs) and other mobile platforms. These platforms may be deployed throughout the facility to transport parts and inventory. This route is also comprised of different multipath from LOS to highly multipath NLOS but with much larger physical range of 200 meters.

The outer loop route measurement runs designated run 1 , run 2 , run 3 , and run 4 started at check point 1 near TX1, as shown in Fig. 4-2(b). The route took the cart away from TX1 and moved towards check point 2, which was $50 \mathrm{~m}$ away, and slowly came back to check point 1 near TX1. In Fig. 4-23, there is a distinct step at $50 \mathrm{~m}$. This step occurs when the cart turns left at check point 2, leaving the LOS condition and entering into a NLOS condition. On the return journey (back to check point 1 ), the cart makes a right turn at check point 12, thus leaving the NLOS condition and entering once again into a LOS condition. This distinct step did not show up in the internal loop run $\mathrm{E}$, run $\mathrm{F}$, run $\mathrm{A}$, and run $\mathrm{B}$, which all had an identical route start. The limited number of measurement paths for a range of around $50 \mathrm{~m}$ for the outer loop route versus the inner loop route leads to this distinct step in the outer route. That is, the only path for $50 \mathrm{~m}$ is the one going from LOS to NLOS for the outer loop. For the inner loop route, the majority of the measurements and check points are all within a $50 \mathrm{~m}$ radius of the transmitting antennas. These additional measurements appear to "muddle" away the distinct step seen in the outer loop route data. Thus, the distinct step from LOS to NLOS does exist in the inner loop route but it cannot be seen due to the increased number of measurements for this route. 


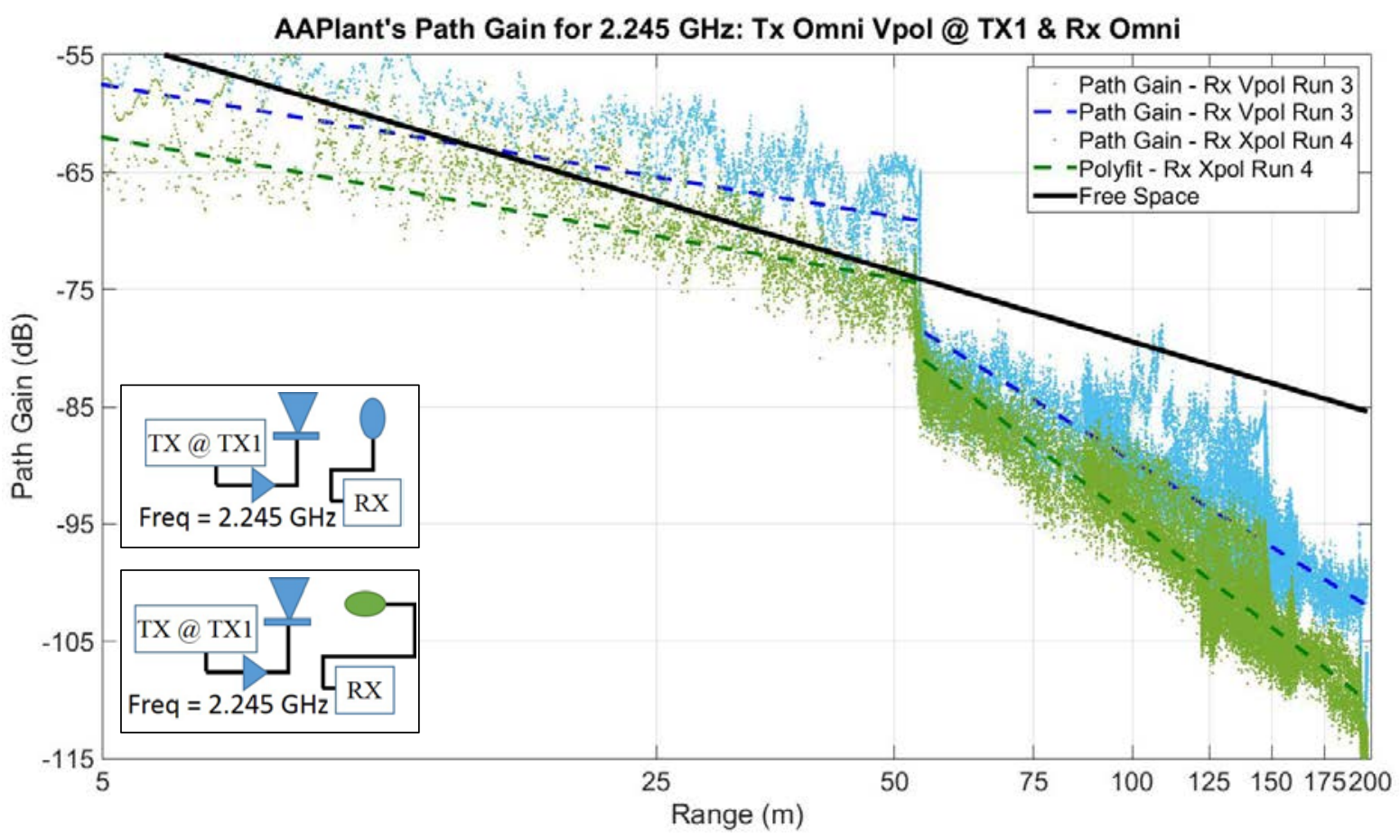

(a)

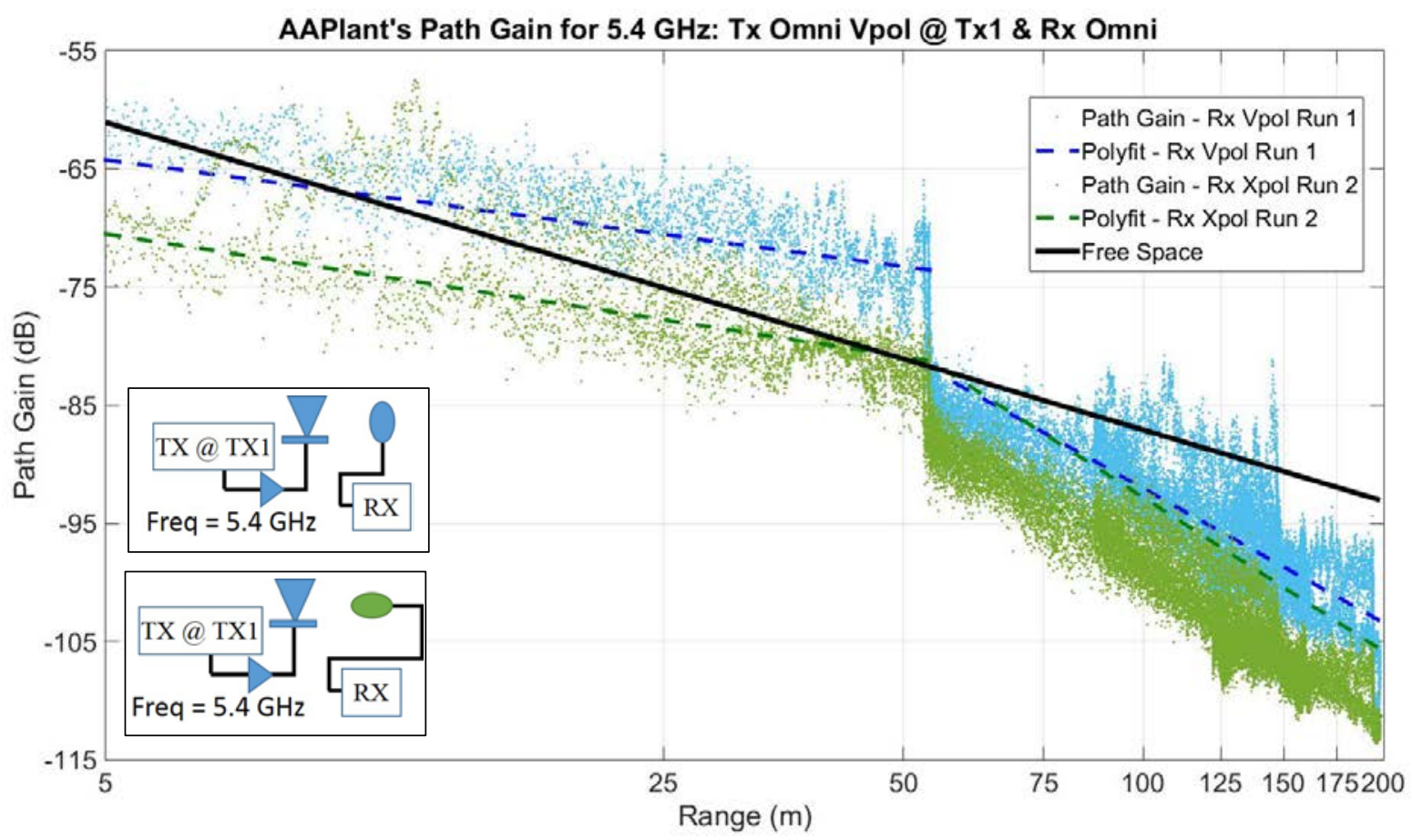

(b)

Fig. 4-23 Path gain measurements in the AAP for Vpol omnidirectional TX antenna at position TX1 for Vpol or Xpol omnidirectional RX antenna: (a) at $2.245 \mathrm{GHz}$ and (b) at $5.4 \mathrm{GHz}$. 


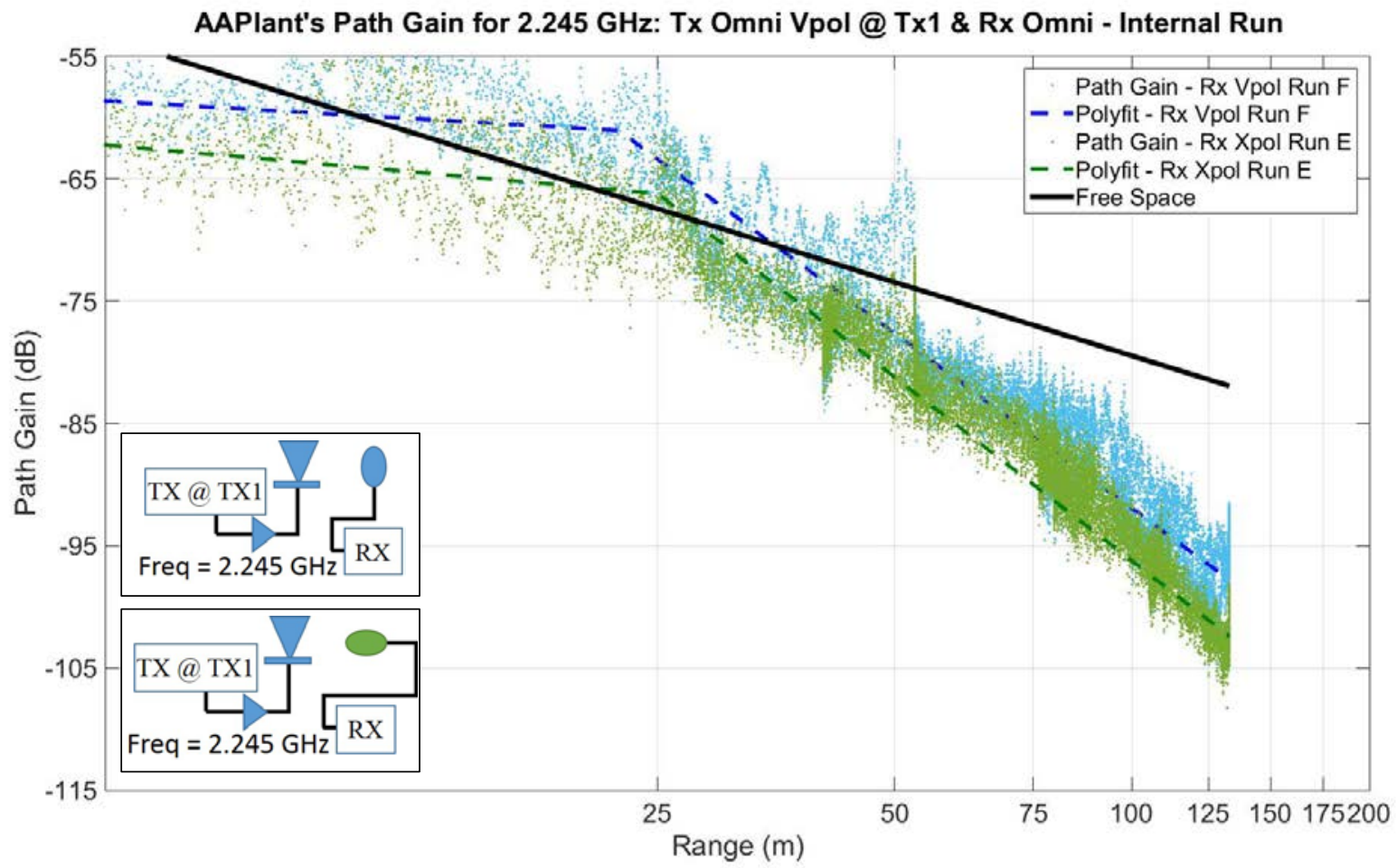

(a)

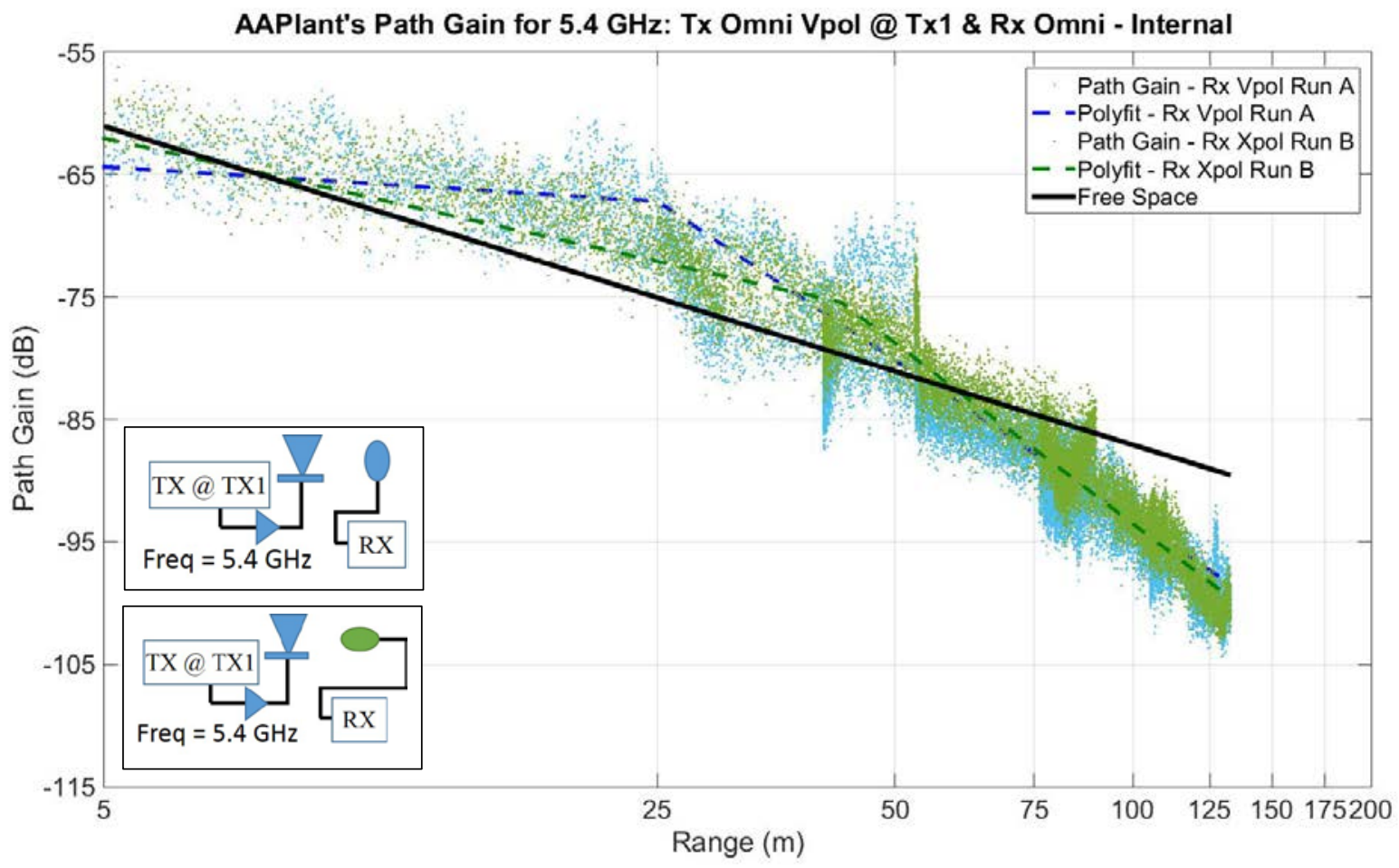

(b)

Fig. 4-24 Path gain measurements in the AAP for Vpol omnidirectional TX antenna at position TX1 for Vpol or Xpol omnidirectional RX antenna: (a) at $2.245 \mathrm{GHz}$ internal run and (b) at $5.4 \mathrm{GHz}$ internal run. 


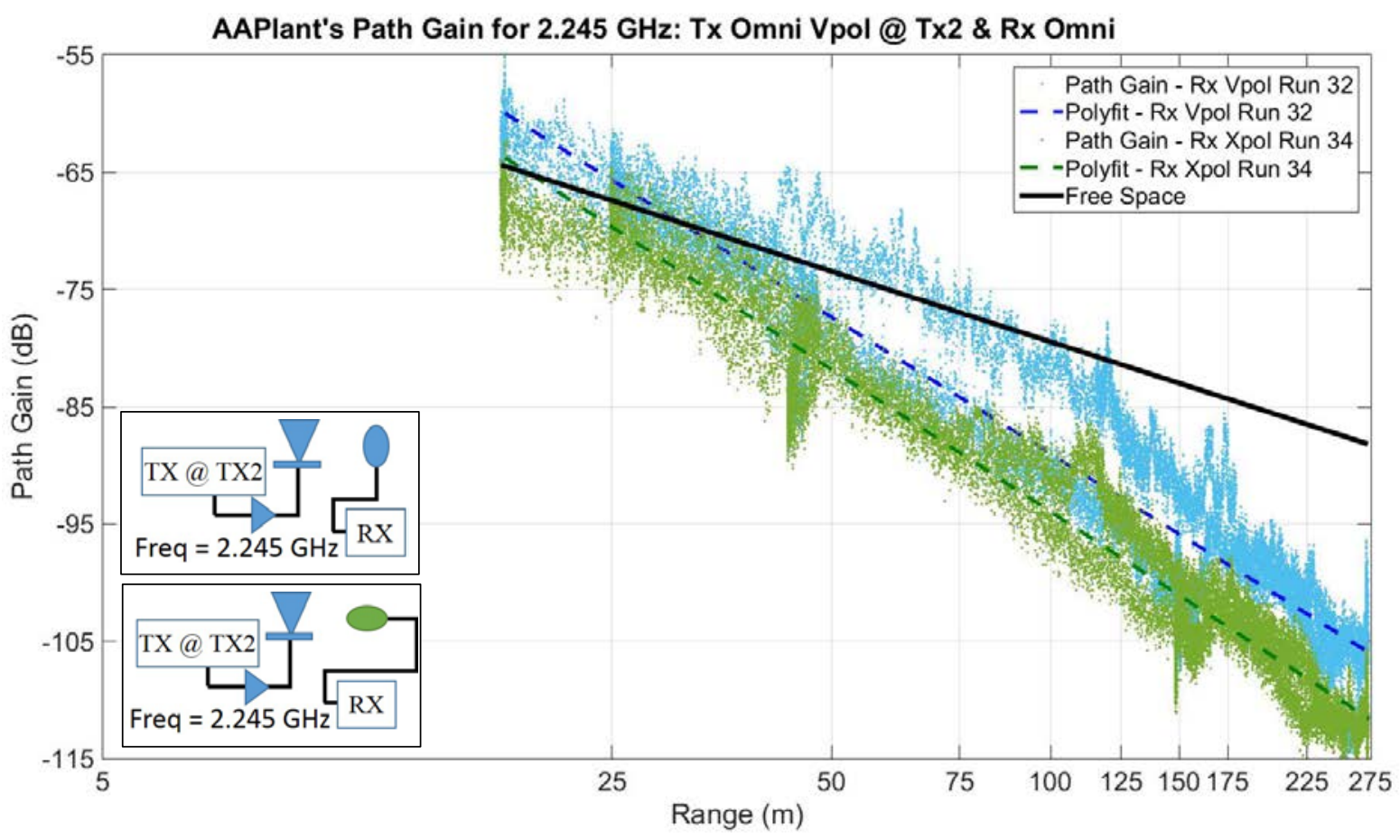

(a)

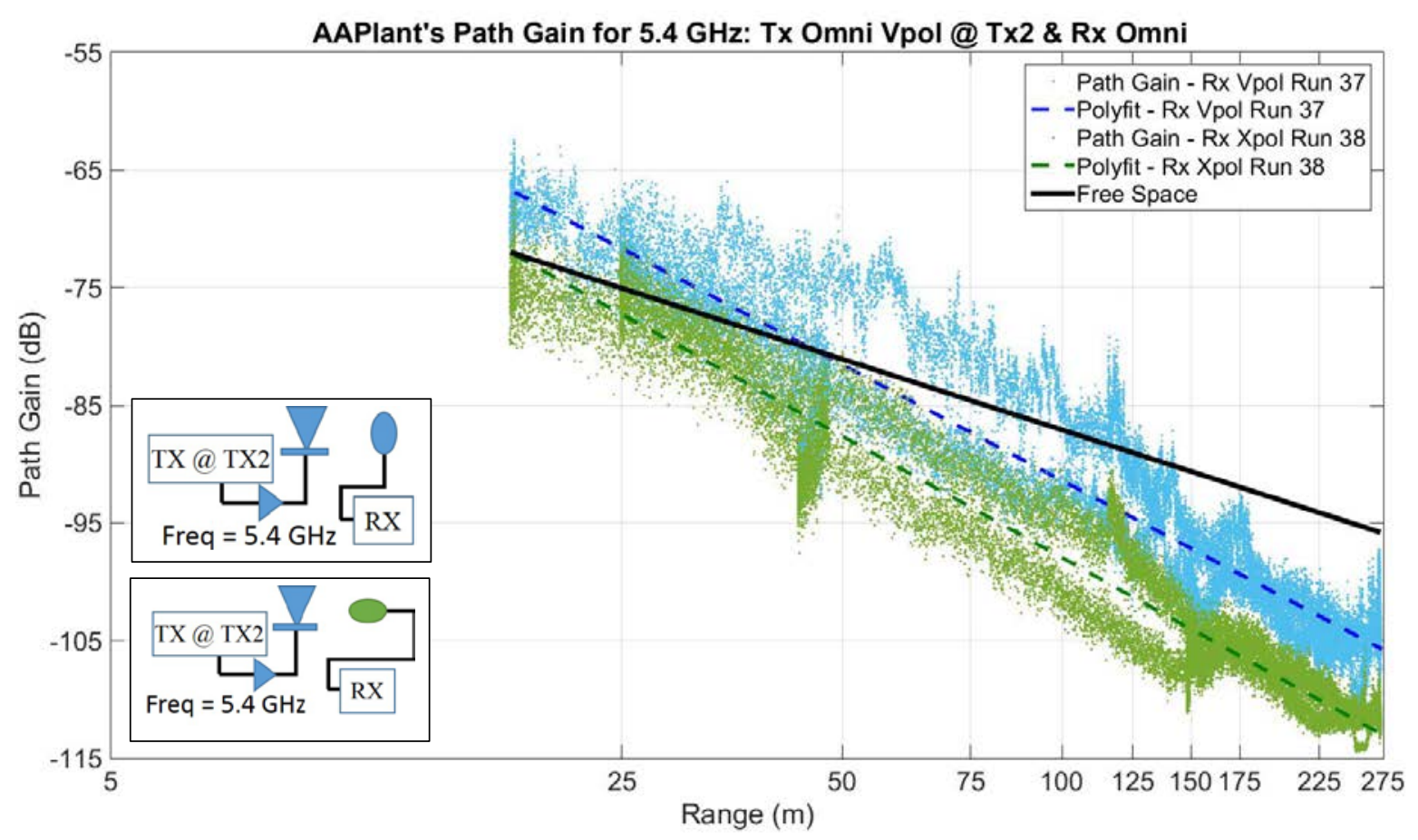

(b)

Fig. 4-25 Path gain measurements in the AAP for Vpol omnidirectional TX antenna at position TX2 for Vpol or Xpol omnidirectional RX antenna: (a) at $2.245 \mathrm{GHz}$ and (b) at $5.4 \mathrm{GHz}$. 


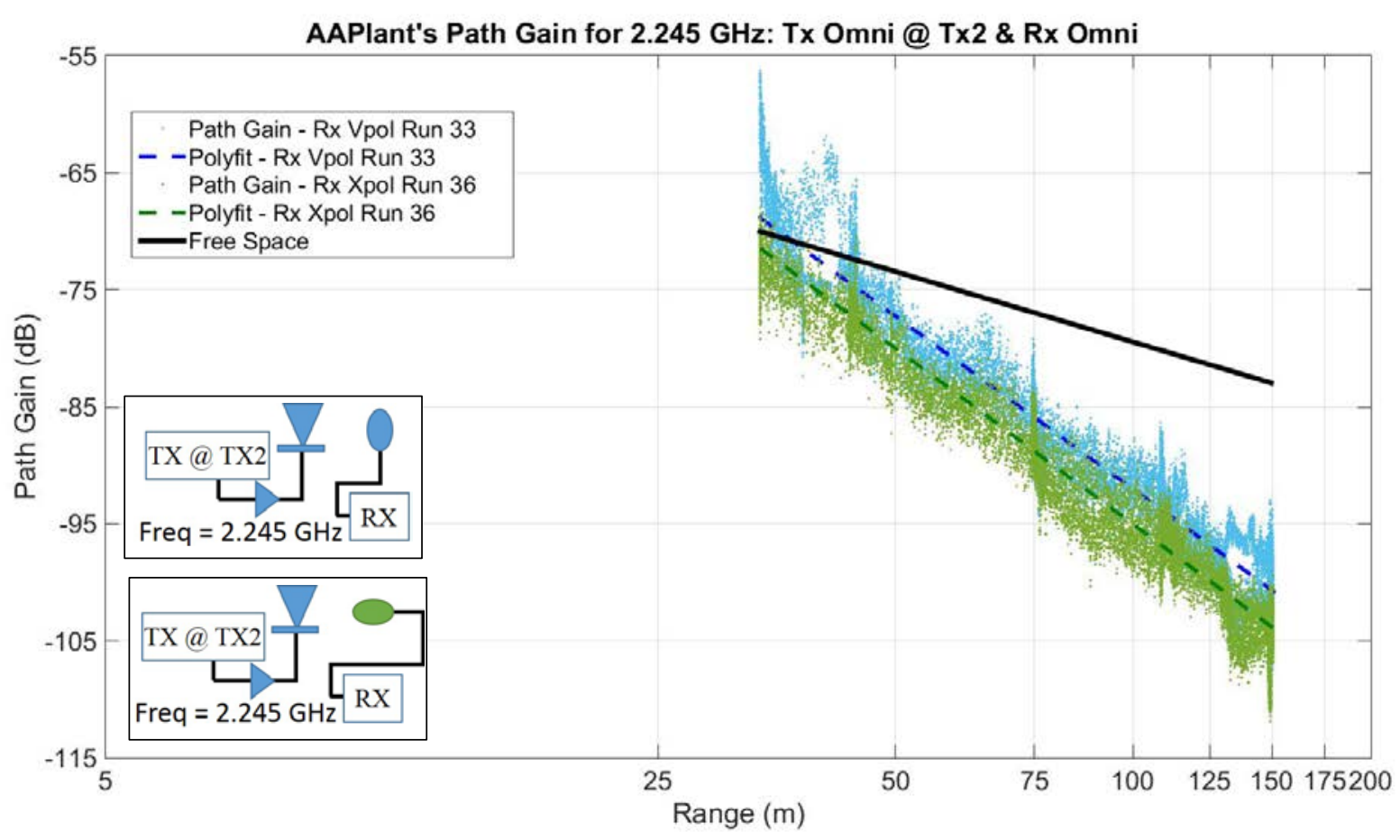

(a)

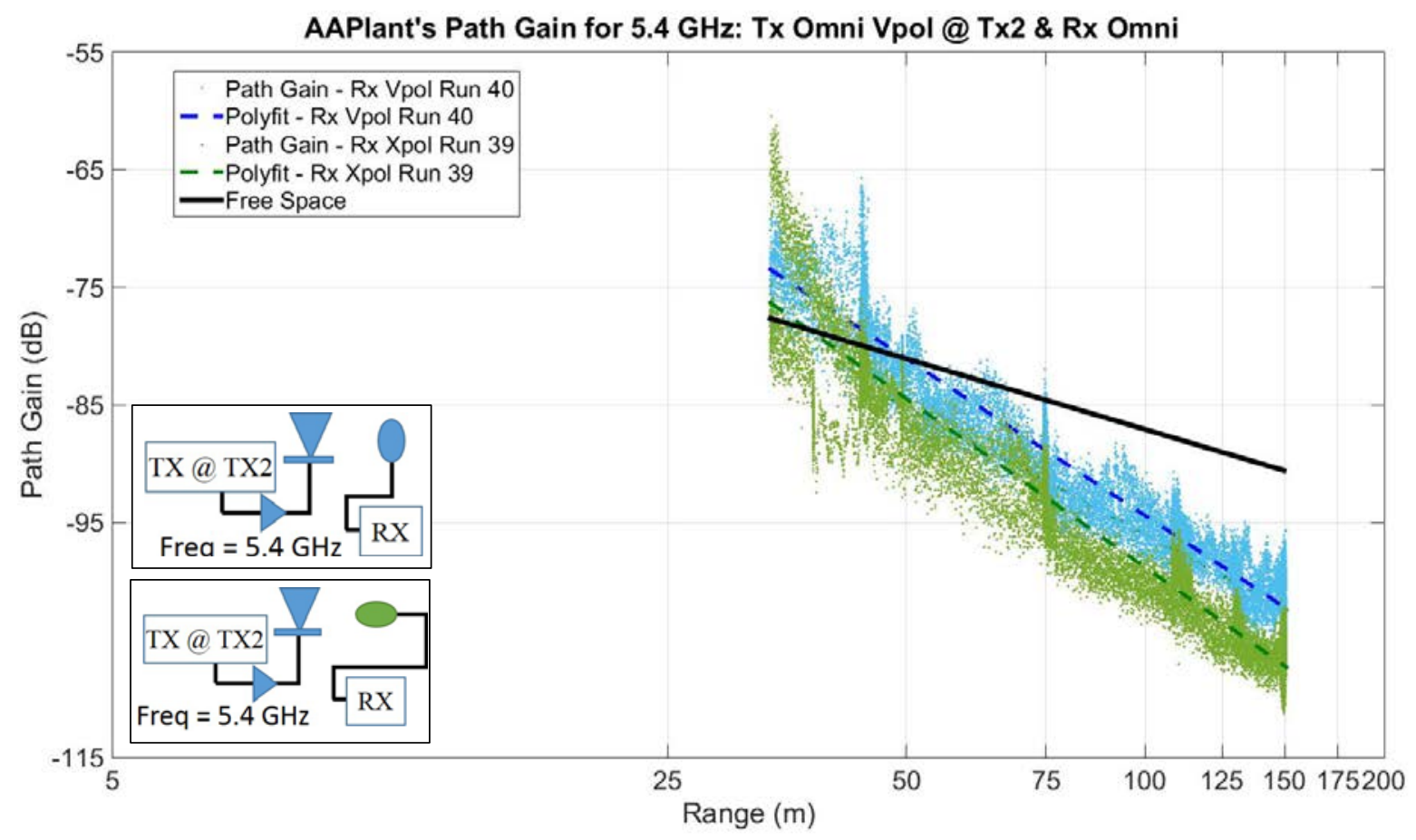

(b)

Fig. 4-26 Path gain measurements in the AAP for Vpol omnidirectional TX antenna at position TX2 for Vpol or Xpol omnidirectional RX antenna: (a) at $2.245 \mathrm{GHz}$ internal run and (b) at $5.4 \mathrm{GHz}$ internal run. 


\subsection{NIST-Gaithersburg, MD Machine Shop Measurement Campaign}

The NIST Gaithersburg Machine Shop (Gburg) is located on the NIST campus in Gaithersburg, MD. This shop is a prototype fabrication facility that manufactures parts for use by NIST researchers. The shop contains several fabrication machine tools such as a Computer Numerical Control (CNC) milling machine, CNC turning and inspection equipment such as Coordinate Measurement Machine (CMM), and digital micrometers. The NIST shop is similar to many machine shops located throughout the U.S. The floor dimensions of the shop are approximately $12 \mathrm{~m} \times 15 \mathrm{~m}$ with a ceiling height of $7.6 \mathrm{~m}$. The transmitter positions are given in Table 4-7, with reference to the origin (orange circle) shown in Fig. 4-27. The antennas types are described in Section 2.1.

Table 4-6: NIST-Gaithersburg, MD machine shop TX locations.

\begin{tabular}{|c|c|c|c|}
\hline TX Location & X(m) & Y(m) & Z(m) \\
\hline TX1 & 34.19 & 60.27 & 3.07 \\
\hline TX2low & 18.31 & 38.89 & 3.07 \\
\hline TX2high & 18.31 & 38.89 & 4.15 \\
\hline
\end{tabular}

Th measurement run for the Gaithersburg machine shop route seen in Fig. 4-27. This route is comprised of different multipaths from LOS to highly multipaths NLOS in a physically smaller footprint.

We conducted the channel-sounder measurements for this campaign during the spring of 2015 using the previously described channel-sounder system with a symbol rate of $20 \mathrm{Msym} / \mathrm{s}, 4$ $\frac{\text { Samples }}{\text { symbol }}$, and a PN code length of 2047. The attenuation for the back-to-back measurement was 50 $\mathrm{dB}$. The post processing of the measurement data used a PNideal filter with discrete sum scaling.

A number of measurement runs were conducted during the Gburg measurement campaign and the measurement data may be downloaded from the website, http://doi.org/10.18434/T44S3N. 
Table 4-7: Gaithersburg machine shop runs.

\begin{tabular}{|c|c|c|c|c|c|c|}
\hline Measurement Run & $\begin{array}{l}\text { Freq. } \\
\text { (GHz) }\end{array}$ & $\begin{array}{c}\text { TX } \\
\text { Position }\end{array}$ & $\begin{array}{c}\text { Path Gain Model } \\
\text { B, A(dB) }\end{array}$ & $\begin{array}{l}\text { Acquisitio } \\
\text { n Numbers } \\
\text { (start, end) }\end{array}$ & $\begin{array}{c}\text { Figure } \\
\text { \#s }\end{array}$ & $\begin{array}{l}\text { Measurement } \\
\text { Filename }\end{array}$ \\
\hline $\begin{array}{l}\text { RX: Vertical Pol. } \\
\text { TX: Vertical Pol. } \\
\text { Omnidirectional }\end{array}$ & 2.245 & TX1 & $\begin{array}{c}r_{B P}=11 \\
\mathrm{~B} 1=-0.27, \mathrm{~A} 1=-52 \\
\mathrm{~B} 2=-2.8, \mathrm{~A} 2=-27\end{array}$ & 20,200 & $\begin{array}{l}\text { Fig. } \\
4-28(a)\end{array}$ & $\begin{array}{c}\text { GBurgD2_2GHz_T } \\
\text { X1_vpol_run1 }\end{array}$ \\
\hline $\begin{array}{l}\text { RX: Horizontal Pol. } \\
\text { TX: Vertical Pol. } \\
\text { Omnidirectional }\end{array}$ & 2.245 & TX1 & $\begin{array}{c}r_{B P}=11 \\
\mathrm{~B} 1=-0.04, \mathrm{~A} 1=-61 \\
\mathrm{~B} 2=-2.2, \mathrm{~A} 2=-38\end{array}$ & 27,215 & $\begin{array}{l}\text { Fig. } \\
\text { 4-28(a) }\end{array}$ & $\begin{array}{l}\text { GBurgD2_2GHz_T } \\
\text { X1_crossPol_run3 }\end{array}$ \\
\hline $\begin{array}{l}\text { RX: Horizontal Long Pol. } \\
\text { TX: Vertical Pol. } \\
\text { Omnidirectional }\end{array}$ & 2.245 & TX1 & $\begin{array}{c}r_{B P}=11 \\
\mathrm{~B} 1=-0.26, \mathrm{~A} 1=-58 \\
\mathrm{~B} 2=-2.3, \mathrm{~A} 2=-38\end{array}$ & 10, 196 & $\begin{array}{l}\text { Fig. } \\
\text { 4-28(a) }\end{array}$ & $\begin{array}{c}\text { GBurgD2_2GHz_T } \\
\text { X1_longPol_run2 }\end{array}$ \\
\hline $\begin{array}{l}\text { RX: Vertical Pol. } \\
\text { TX: Vertical Pol. } \\
\text { Omnidirectional }\end{array}$ & 5.4 & TX1 & $\begin{aligned} & r_{B P}=11 \\
\mathrm{~B} 1= & 0.26, \mathrm{~A} 1=-64 \\
\mathrm{~B} 2= & -2.7, \mathrm{~A} 2=-34\end{aligned}$ & 7, 211 & $\begin{array}{l}\text { Fig. } \\
\text { 4-28(b) }\end{array}$ & $\begin{array}{c}\text { GBurgD2_5GHz_T } \\
\text { X1_vpol_run2 }\end{array}$ \\
\hline $\begin{array}{l}\text { RX: Horizontal Pol. } \\
\text { TX: Vertical Pol. } \\
\text { Omnidirectional }\end{array}$ & 5.4 & TX1 & $\begin{array}{c}r_{B P}=11 \\
\mathrm{~B} 1=-0.04, \mathrm{~A} 1=-70 \\
\mathrm{~B} 2=-2.5, \mathrm{~A} 2=-44\end{array}$ & 25,223 & $\begin{array}{l}\text { Fig. } \\
\text { 4-28(b) }\end{array}$ & $\begin{array}{l}\text { GBurgD2_5GHz_T } \\
\text { X1_crossPol_run3 }\end{array}$ \\
\hline $\begin{array}{l}\text { RX: Horizontal Long Pol. } \\
\text { TX: Vertical Pol. } \\
\text { Omnidirectional }\end{array}$ & 5.4 & TX1 & $\begin{array}{c}r_{B P}=11 \\
\mathrm{~B} 1=-0.28, \mathrm{~A} 1=-67 \\
\mathrm{~B} 2=-2.3, \mathrm{~A} 2=-46\end{array}$ & 30,214 & $\begin{array}{l}\text { Fig. } \\
\text { 4-28(b) }\end{array}$ & $\begin{array}{c}\text { GBurgD2_5GHz_T } \\
\text { X1_longPol_run4 }\end{array}$ \\
\hline $\begin{array}{l}\text { RX: Vertical Pol. } \\
\text { TX: Vertical Pol. } \\
\text { Omnidirectional } \\
\end{array}$ & 2.245 & TX2low & $\begin{array}{c}r_{B P}=13 \\
\mathrm{~B} 1=-0.16, \mathrm{~A} 1=-52 \\
\mathrm{~B} 2=-1.8, \mathrm{~A} 2=-41\end{array}$ & 8,214 & $\begin{array}{l}\text { Fig. } \\
\text { 4-29(a) }\end{array}$ & $\begin{array}{l}\text { GBurgD3_2GHz_T } \\
\text { X2low_vpol_run1 }\end{array}$ \\
\hline $\begin{array}{l}\text { RX: Horizontal Pol. } \\
\text { TX: Vertical Pol. } \\
\text { Omnidirectional }\end{array}$ & 2.245 & TX2low & $\begin{array}{c}r_{B P}=13 \\
\mathrm{~B} 1=-1.2, \mathrm{~A} 1=-52 \\
\mathrm{~B} 2=-1.5, \mathrm{~A} 2=-48\end{array}$ & 27,234 & $\begin{array}{l}\text { Fig. } \\
\text { 4-29(a) }\end{array}$ & $\begin{array}{c}\text { GBurgD3_2GHz_T } \\
\text { X2low_crossPol_ru } \\
\text { n2 }\end{array}$ \\
\hline $\begin{array}{l}\text { RX: Horizontal Long Pol. } \\
\text { TX: Vertical Pol. } \\
\text { Omnidirectional }\end{array}$ & 2.245 & TX2low & $\begin{array}{c}r_{B P}=13 \\
\mathrm{~B} 1=1.1, \mathrm{~A} 1=-75 \\
\mathrm{~B} 2=-1.3, \mathrm{~A} 2=-51\end{array}$ & 12,227 & $\begin{array}{l}\text { Fig. } \\
\text { 4-29(a) }\end{array}$ & $\begin{array}{c}\text { GBurgD3_2GHz_T } \\
\text { X2low_longPol_run } \\
3\end{array}$ \\
\hline $\begin{array}{l}\text { RX: Vertical Pol. } \\
\text { TX: Vertical Pol. } \\
\text { Omnidirectional }\end{array}$ & 5.4 & TX2low & $\begin{array}{c}r_{B P}=13 \\
\mathrm{~B} 1=-0.71, \mathrm{~A} 1=-58 \\
\mathrm{~B} 2=-1.3, \mathrm{~A} 2=-53\end{array}$ & 12, 205 & $\begin{array}{l}\text { Fig. } \\
\text { 4-29(b) }\end{array}$ & $\begin{array}{l}\text { GBurgD2_5GHz_T } \\
\text { X2low_vpol_run1 }\end{array}$ \\
\hline $\begin{array}{l}\text { RX: Horizontal Pol. } \\
\text { TX: Vertical Pol. } \\
\text { Omnidirectional }\end{array}$ & 5.4 & TX2low & $\begin{array}{c}r_{B P}=13 \\
\mathrm{~B} 1=-0.97, \mathrm{~A} 1=-64 \\
\mathrm{~B} 2=-1.2, \mathrm{~A} 2=-60\end{array}$ & 21,225 & $\begin{array}{l}\text { Fig. } \\
\text { 4-29(b) }\end{array}$ & $\begin{array}{l}\text { GBurgD2_5GHz_T } \\
\text { X2low_crossPol }\end{array}$ \\
\hline $\begin{array}{l}\text { RX: Horizontal Long Pol. } \\
\text { TX: Vertical Pol. } \\
\text { Omnidirectional }\end{array}$ & 5.4 & TX2low & $\begin{array}{c}r_{B P}=13 \\
\mathrm{~B} 1=-0.99, \mathrm{~A} 1=-63 \\
\mathrm{~B} 2=-1.5, \mathrm{~A} 2=-57\end{array}$ & 36, 242 & $\begin{array}{l}\text { Fig. } \\
\text { 4-29(b) }\end{array}$ & $\begin{array}{l}\text { GBurgD2_5GHz_T } \\
\text { X2low_longPol }\end{array}$ \\
\hline $\begin{array}{l}\text { RX: Vertical Pol. } \\
\text { TX: Vertical Pol. } \\
\text { Omnidirectional }\end{array}$ & 2.245 & TX2high & $\begin{array}{c}r_{B P}=13 \\
\mathrm{~B} 1=-0.61, \mathrm{~A} 1=-53 \\
\mathrm{~B} 2=-1.5, \mathrm{~A} 2=-44\end{array}$ & 16, 204 & $\begin{array}{l}\text { Fig. } \\
\text { 4-30(a) }\end{array}$ & $\begin{array}{l}\text { GburgD3_2GHz_T } \\
\text { X2high_vpol_run1 }\end{array}$ \\
\hline $\begin{array}{l}\text { RX: Horizontal Pol. } \\
\text { TX: Vertical Pol. } \\
\text { Omnidirectional } \\
\end{array}$ & 2.245 & TX2high & $\begin{array}{c}r_{B P}=13 \\
\mathrm{~B} 1=-0.8, \mathrm{~A} 1=-56 \\
\mathrm{~B} 2=-1.5, \mathrm{~A} 2=-48\end{array}$ & 14, 211 & $\begin{array}{l}\text { Fig. } \\
\text { 4-30(a) }\end{array}$ & $\begin{array}{c}\text { GBurgD3_2GHz_T } \\
\text { X2high_crossPol_ru } \\
\text { n2 } \\
\end{array}$ \\
\hline $\begin{array}{l}\text { RX: Horizontal Long Pol. } \\
\text { TX: Vertical Pol. } \\
\text { Omnidirectional }\end{array}$ & 2.245 & TX2high & $\begin{array}{c}r_{B P}=13 \\
\mathrm{~B} 1=-0.73, \mathrm{~A} 1=-56 \\
\mathrm{~B} 2=-1.6, \mathrm{~A} 2=-47\end{array}$ & 25,230 & $\begin{array}{l}\text { Fig. } \\
\text { 4-30(a) }\end{array}$ & $\begin{array}{c}\text { GBurgD3_2GHz_T } \\
\text { X2high_longPol_ru } \\
\text { n3 }\end{array}$ \\
\hline $\begin{array}{l}\text { RX: Vertical Pol. } \\
\text { TX: Vertical Pol. } \\
\text { Omnidirectional }\end{array}$ & 5.4 & TX2high & $\begin{array}{c}r_{B P}=13 \\
\mathrm{~B} 1=-0.7, \mathrm{~A} 1=-60 \\
\mathrm{~B} 2=-1.0, \mathrm{~A} 2=-57\end{array}$ & 29,227 & $\begin{array}{l}\text { Fig. } \\
\text { 4-30(b) }\end{array}$ & $\begin{array}{l}\text { GburgD2_5GHz_T } \\
\text { X2high_vpol }\end{array}$ \\
\hline $\begin{array}{l}\text { RX: Horizontal Pol. } \\
\text { TX: Vertical Pol. } \\
\text { Omnidirectional }\end{array}$ & 5.4 & TX2high & $\begin{array}{c}r_{B P}=13 \\
\mathrm{~B} 1=-0.97, \mathrm{~A} 1=-63 \\
\mathrm{~B} 2=-1.1, \mathrm{~A} 2=-61\end{array}$ & 19, 221 & $\begin{array}{l}\text { Fig. } \\
\text { 4-30(b) }\end{array}$ & $\begin{array}{l}\text { GBurgD2_5GHz_T } \\
\text { X2high_crossPol }\end{array}$ \\
\hline $\begin{array}{l}\text { RX: Horizontal Long Pol. } \\
\text { TX: Vertical Pol. } \\
\text { Omnidirectional }\end{array}$ & 5.4 & TX2high & $\begin{array}{c}r_{B P}=13 \\
\mathrm{~B} 1=-0.73, \mathrm{~A} 1=-65 \\
\mathrm{~B} 2=-1.1, \mathrm{~A} 2=-61\end{array}$ & 15, 221 & $\begin{array}{l}\text { Fig. } \\
\text { 4-30(b) }\end{array}$ & $\begin{array}{l}\text { GBurgD2_5GHz_T } \\
\text { X2high_longPol }\end{array}$ \\
\hline
\end{tabular}




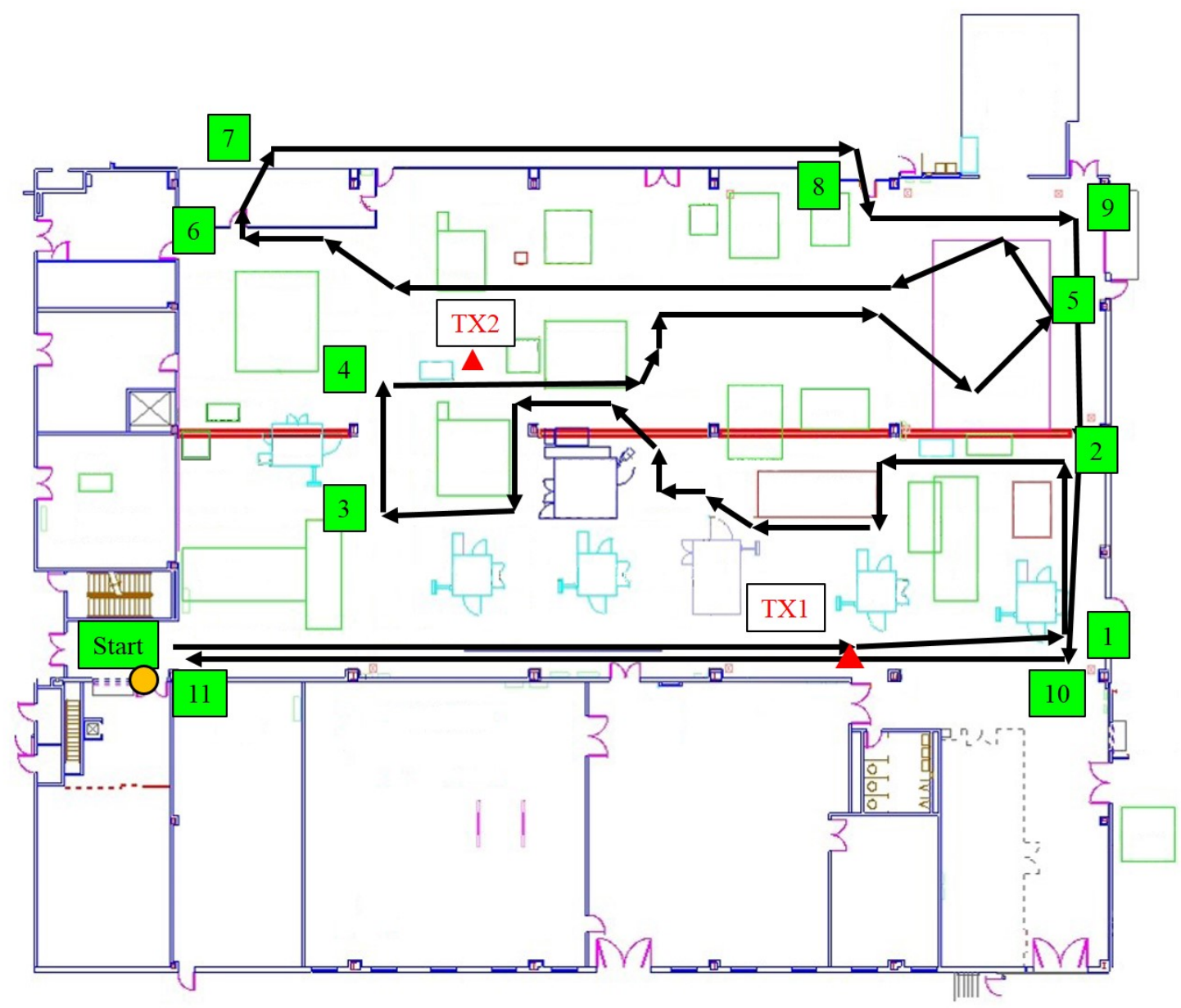

Fig. 4-27: Measurement route through the Gaithersburg machine shop.

The loop route using TX1 has slopes B2 comparable to the CUP measurement campaign of -2.8 and $-2.7 \mathrm{~dB} / \mathrm{m}$ for $2.245 \mathrm{GHz}$ and $5.4 \mathrm{GHz}$, respectively. For the loop route measurement runs with Tx2low and Tx2high, the low and high are a reference to the transmitting antenna height, as seen in Table 4-6. The slope for B2 for Tx2low and Tx2high for frequency of $2.245 \mathrm{GHz}$ at a break point of $13 \mathrm{~m}$ is $-1.8 \mathrm{~dB} / \mathrm{m}$ and $-1.5 \mathrm{~dB} / \mathrm{m}$. While for the frequency of $5.4 \mathrm{GHz}$, the slopes are $-1.3 \mathrm{~dB} / \mathrm{m}$ and $-1.0 \mathrm{~dB} / \mathrm{m}$ at a break point of $13 \mathrm{~m}$. These slopes are comparable to the CUP measurement slopes for similar cases while the AAP measurement slopes are considerably larger. 


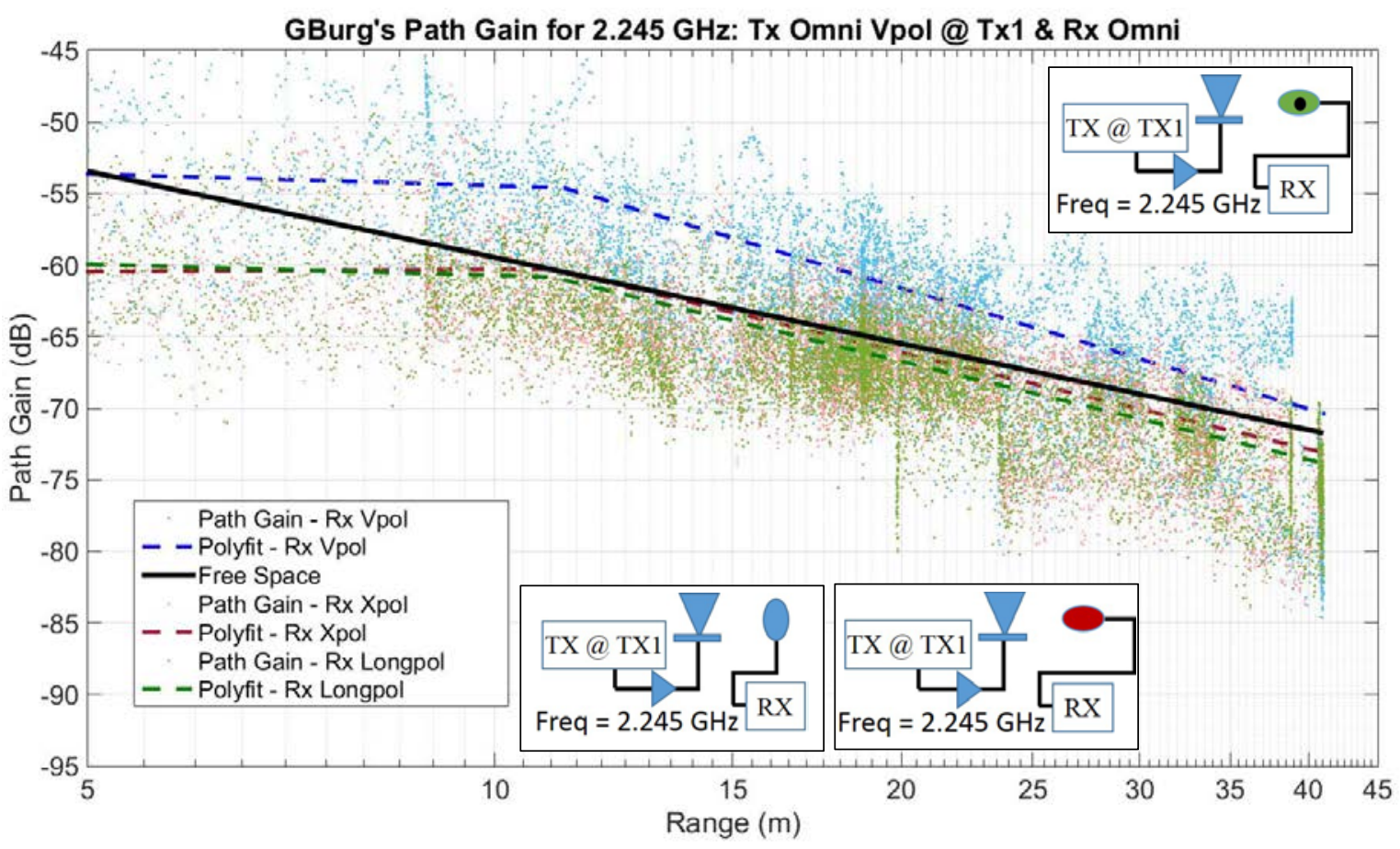

(a)

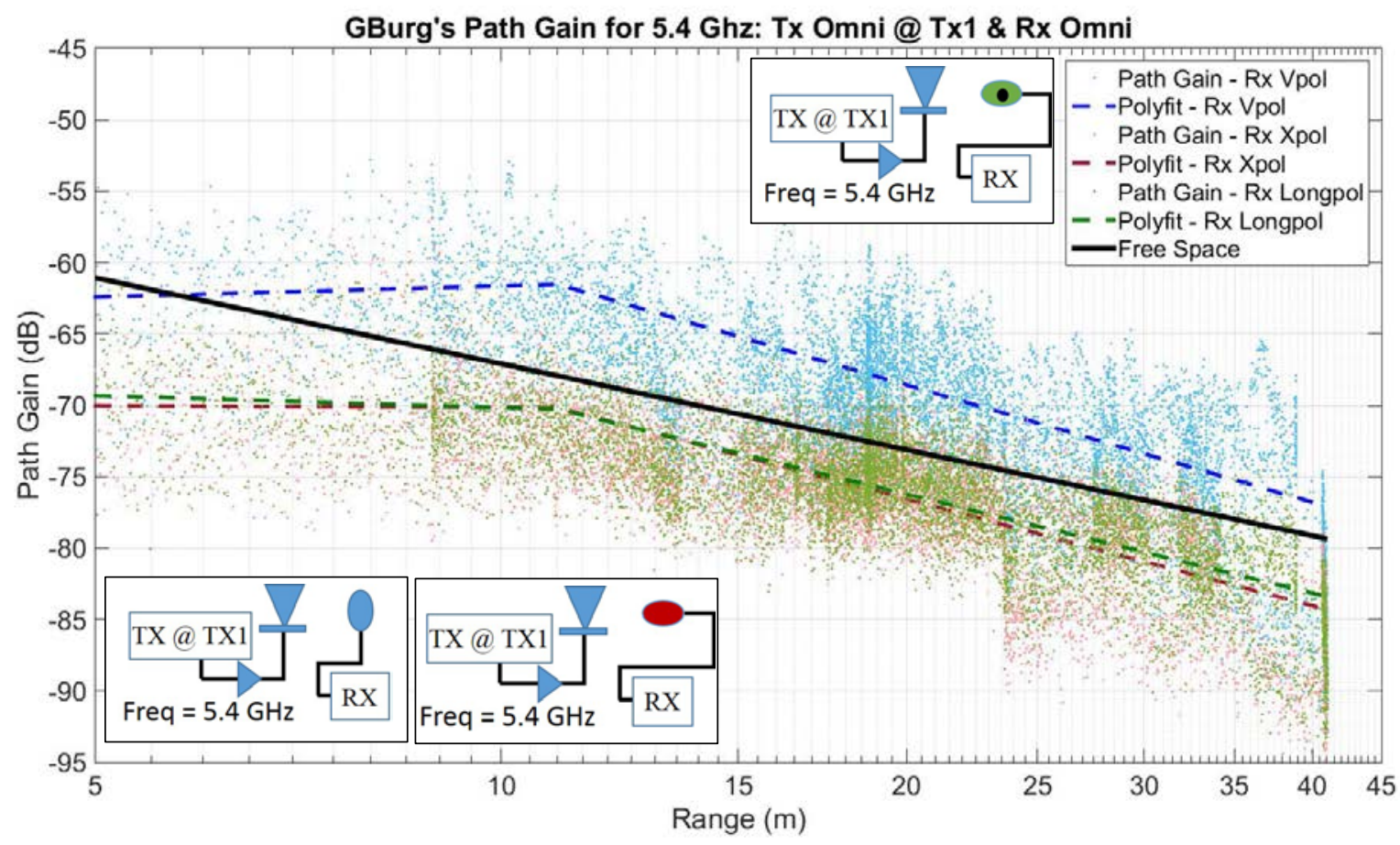

(b)

Fig. 4-28 Path gain measurements in the Gaithersburg (GBurg) Machine Shop for Vpol omnidirectional TX antenna at position TX1 for Vpol or Xpol or Longpol omnidirectional RX antenna: (a) at $2.245 \mathrm{GHz}$ and (b) at $5.4 \mathrm{GHz}$. 


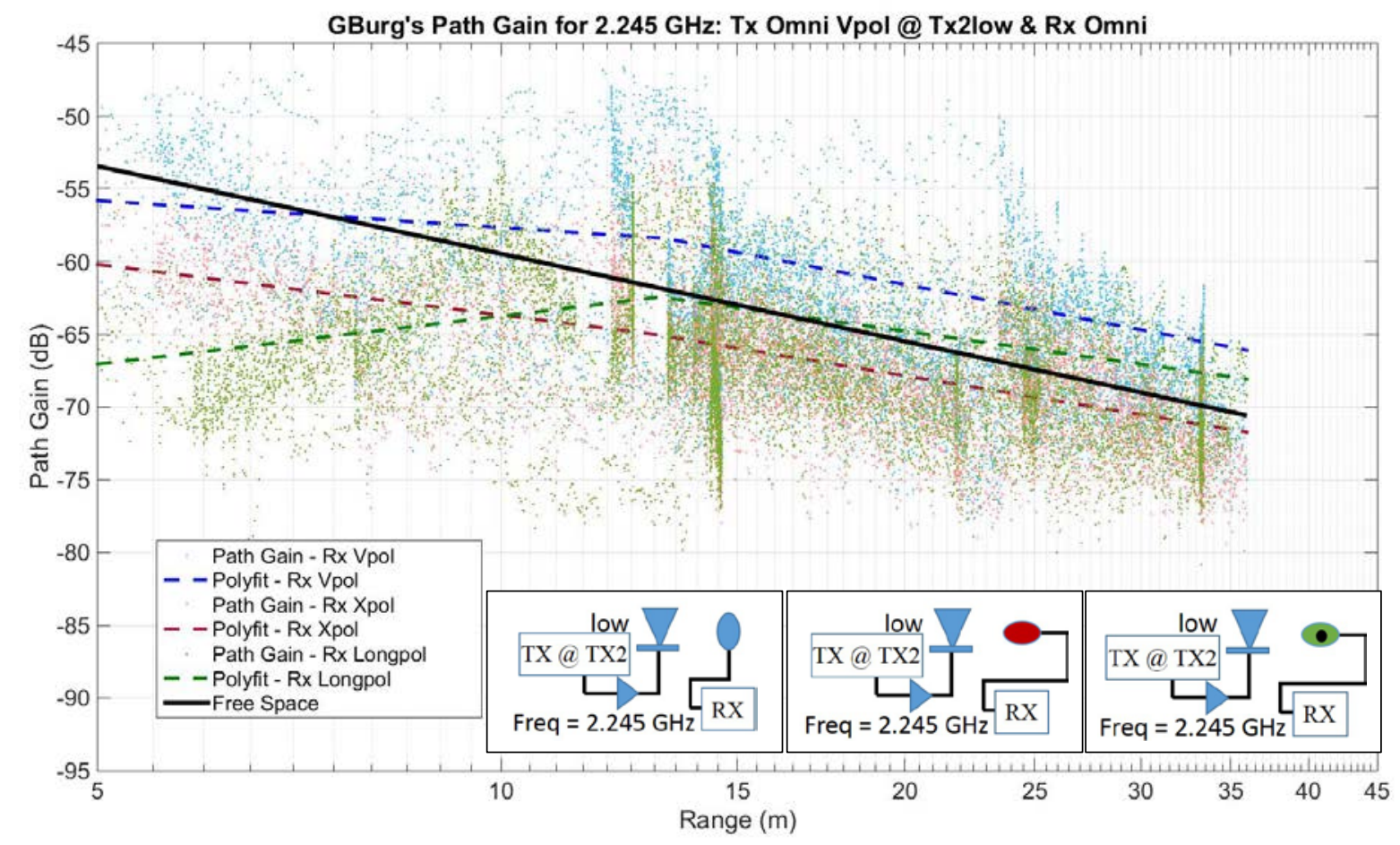

(a)

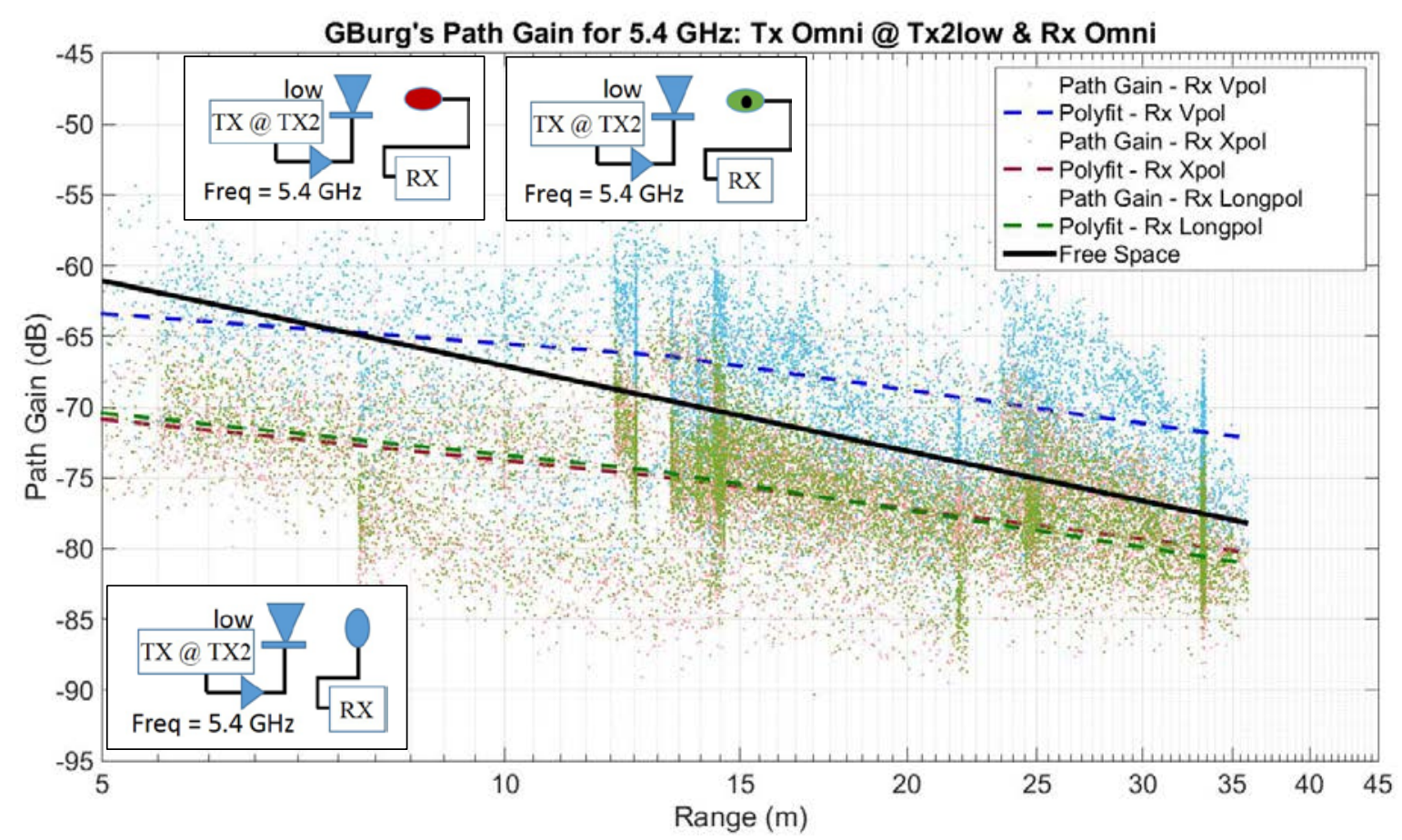

(b)

Fig. 4-29 Path gain measurements in the Gaithersburg (GBurg) Machine Shop for Vpol omnidirectional TX antenna at position TX2low for Vpol or Xpol or Longpol omnidirectional RX antenna: (a) at $2.245 \mathrm{GHz}$ and (b) at $5.4 \mathrm{GHz}$. 


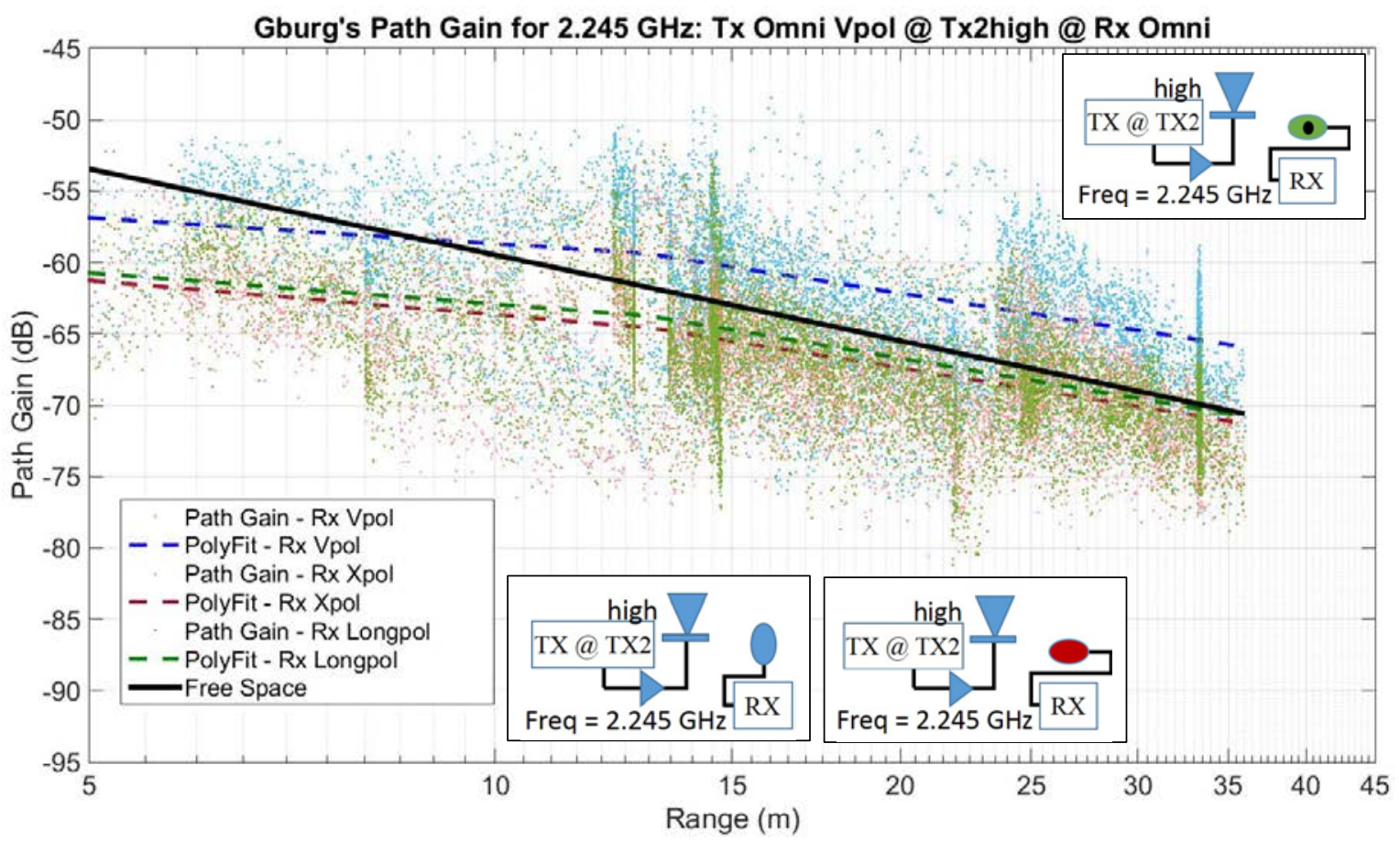

(a)

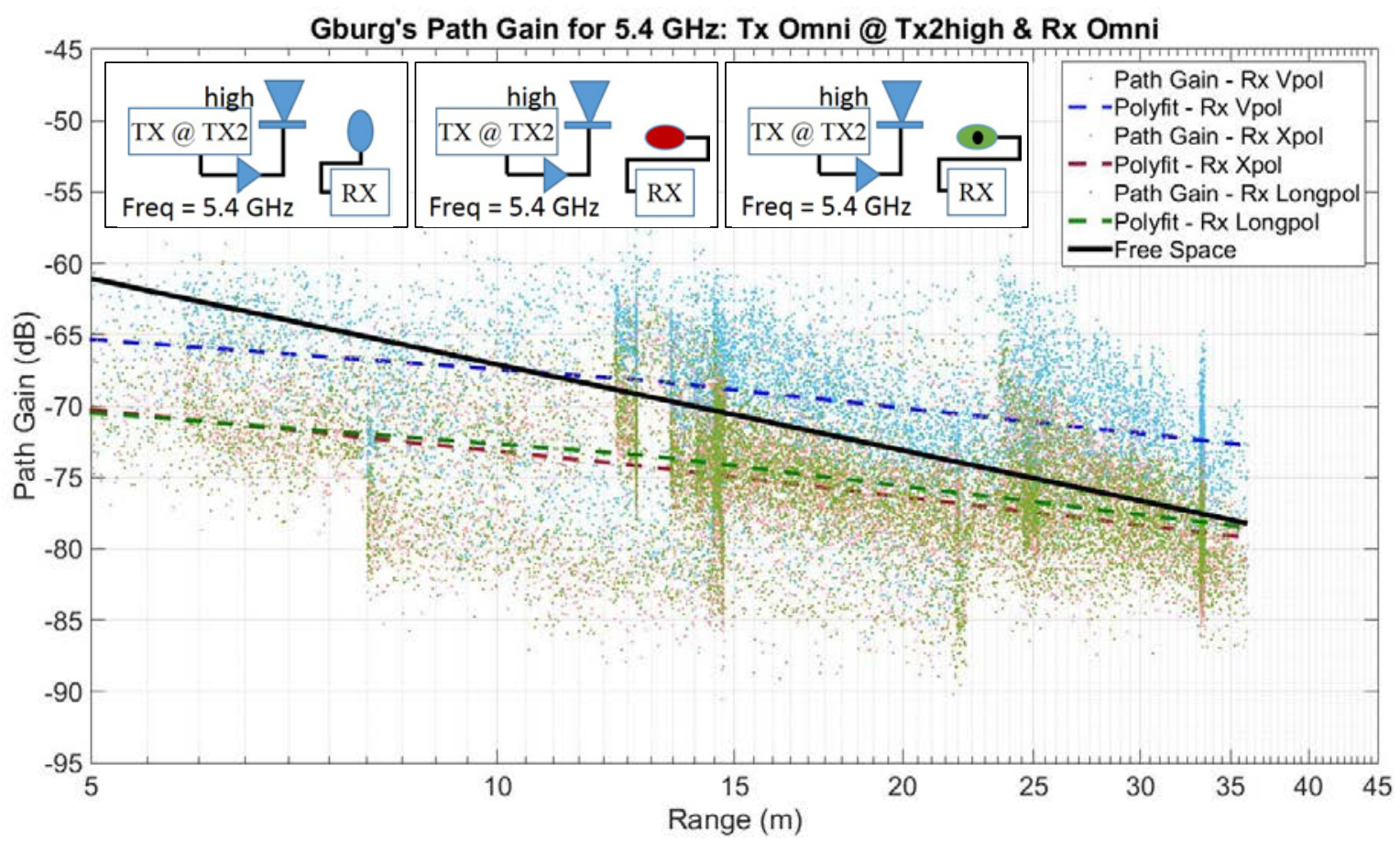

(b)

Fig. 4-30 Path gain measurements in the Gaithersburg (GBurg) Machine Shop for Vpol omnidirectional TX antenna at position TX2high for Vpol or Xpol or Longpol omnidirectional RX antenna: (a) at $2.245 \mathrm{GHz}$ and (b) at $5.4 \mathrm{GHz}$. 


\section{Conclusion}

The deployment of wireless technologies in industrial manufacturing environments requires an indepth understanding of the propagation environment. Environments such as oil refineries or automotive plants are difficult propagation environments due to the highly reflective materials that induce scattering phenomena.

Characterization of these environments was provided through three measurement campaigns. These measurement campaigns include a central utility steam plant, an automotive assembly plants and a machine shop. The measurements reported here consisted of mobile measurement and fixedposition data acquired with a PN-sequence correlation-based channel sounder operating in the frequency range of $65 \mathrm{MHz}$ to $6 \mathrm{GHz}$ with up to $200 \mathrm{MHz}$ bandwidth. The channel sounder consists of a single transmitter and a single receiver coordinated with two synchronized rubidium clock. The measurement campaign data are available for download at http://doi.org/10.18434/T44S3N. Please reference the DOI when downloading the data for research and/or publications.

Path gain versus range for mobile and fixed measurements are provided in this report. From analysis of these results, a few summary comments may be made about industrial environments.

Comment 1: The slopes from the linear regression fits for large-range values agreed with previous studies and research for industrial facilities environments [13, 14, 15,16,17].

Comment 2: For transmitters located near the receiver location, the effect of the antenna radiation pattern has more influence on the power received than for receiver locations far from the transmitter. This decreased influence may be attributed to an increased number of multipath components which help the power reach the receiver antenna using many different paths.

Comment 3: Due to the influence of the antenna patterns, the transmitter location near a potential receiver must be carefully considered to make sure that nearby structures are not impeding the propagation signal. This was the case for the CUP measurement campaign where the transmitter was located on an upper level (at TX3) which was blocked from receive locations directly below (receiver check points of 34,35 , and 36 ).

Comment 4: Polarization had a great effect on the power received by the channel sounder. The RX antenna with vertical polarization generally received more power than the horizontallypolarized and cross-polarized antenna orientations.

The measurement campaign data provided here are intended to aid in the deployment of wireless technologies in factory environments. Through the use of real-world measurements, the effects on wireless data communications, reliability and throughput can be modeled through physicsbased modeling to correlate real-world effects on these parameters. Additionally, best-practice guidelines for secure wireless platforms for dynamic production systems may be developed by analyzing wireless technology and protocols that will enable manufacturers, technology providers, and solution providers to design, deploy, and assess robust, secure integrated wireless solutions for use in smart manufacturing. 


\section{Appendix A - List of Equipment}

\subsection{Transmitter (TX) System Equipment}

Table 6-1: Transmitter system equipment.

\begin{tabular}{|l|c|l|l|}
\hline \multicolumn{1}{|c|}{ Name } & Quantity & \multicolumn{1}{c|}{ Manufacturer } & \multicolumn{1}{c|}{ Description } \\
\hline PXIe-1085 & 1 & $\begin{array}{l}\text { National } \\
\text { Instruments }\end{array}$ & PXIe-1085, 18 slot 3U Express Chassis, 12 GB/s System BW \\
\hline PXIe-8135 & 1 & $\begin{array}{l}\text { National } \\
\text { Instruments }\end{array}$ & $\begin{array}{l}\text { PXIe-8135 Core i7-3610QE 2.3 GHz Controller, Win 7 (64 } \\
\text { bit) }\end{array}$ \\
\hline PXI-5691 & 2 & $\begin{array}{l}\text { National } \\
\text { Instruments }\end{array}$ & PXI-5691 8 GHz Programmable RF Amplifier Module \\
\hline PXIe-6674T & 1 & $\begin{array}{l}\text { National } \\
\text { Instruments }\end{array}$ & PXIe-6674T Timing Synchronization Module with OCXO \\
\hline PXI-6683H & 1 & $\begin{array}{l}\text { National } \\
\text { Instruments }\end{array}$ & $\begin{array}{l}\text { PXI-6683H GPS, IRIG-B, IEEE 1588 Sync Time Module with } \\
\text { TCXO }\end{array}$ \\
\hline $\begin{array}{l}\text { PXIe-5646R } \\
\text { VST }\end{array}$ & 1 & $\begin{array}{l}\text { National } \\
\text { Instruments }\end{array}$ & PXIe-5646R VST: 200 MHz BW \\
\hline $\begin{array}{l}\text { Power } \\
\text { Amplifier }\end{array}$ & 1 & $\begin{array}{l}\text { OPHIR } \\
\text { 5ow power amplifier, 20 MHz to 6.0 GHz, 5-10W Set }\end{array}$ \\
\hline
\end{tabular}

\subsection{Receiver (RX) System Equipment}

Table 6-2: Receiver system equipment.

\begin{tabular}{|l|c|l|}
\hline \multicolumn{1}{|c|}{ Name } & Quantity & \multicolumn{1}{c|}{ Description } \\
\hline PXIe-1085 & 1 & PXIe-1085, 18 slot 3U Express Chassis, 12 GB/s System BW \\
\hline PXIe-8135 & 1 & PXIe-8135 Core i7-3610QE 2.3 GHz Controller, Win 7 (64 bit) \\
\hline HDD & 1 & 8260, 4-Drive, 3T HDD High Speed Storage Module, Win 7 Only \\
\hline PXI-6683H & 1 & PXI-6683H GPS, IRIG-B, IEEE 1588 Sync Time Module with TCXO \\
\hline PXIe-6674T & 1 & PXIe-6674T Timing Synchronization Module with OCXO \\
\hline PXIe-5646R VST & 1 & PXIe-5646R VST: 200 MHz BW \\
\hline
\end{tabular}

\subsection{Additional Channel Sounder System Equipment}

Table 6-3 Additional channel sounder system equipment.

\begin{tabular}{|c|c|c|}
\hline Name & Quantity & Description \\
\hline PXIe-1082DC & 1 & PXIe-1082DC 8-Slot 3U PXI Express Chassis with DC Power Supply \\
\hline Power Cord & 2 & Power Cord, AC, U. S., 125VAC, 15A \\
\hline DC Power Cord & 1 & DC Power Cord for PXIe-1082DC \\
\hline $\begin{array}{l}\text { Replacement } \\
\text { Power Supply } \\
\text { Fan }\end{array}$ & 1 & Replacement Power Supply Fan Shuttle for PXIe-1082 \\
\hline PMA-1115 & 1 & $\begin{array}{l}\text { PMA-1115: Portable PXI monitor English Keyboard Accessory for 8-Slot PXI } \\
\text { Chassis }\end{array}$ \\
\hline ISO 17025 & 2 & ISO 17025 Accredited Calibration for RF Analyzer/Generator \\
\hline
\end{tabular}




\section{Appendix B - Installation Guide}

This appendix contains guidance on the installation of the LabVIEW signal generation data acquisition software on the equipment from lessons learned during the process. Challenges as well as solutions met during installation will be provided. This installation guide starts with nothing in the PCIe-1085 Chassis. The manufacturer suggests that installation should be done sequentially. First, software drivers are installed for a module then the physical module is inserted into the PCIe-1085 Chassis. This process is carried out on a single module one at a time. This way, if a failure occurs in the installation process it is easier to determine the point of failure.

First, the PXIe-8135 controller is inserted into the PCIe-1085 Receiver Chassis as seen in Fig. 7-1. Next, the monitor, keyboard, power cables are connected to the PCIe-1085 Receiver Chassis. The PCIe-8135 controller is turned on. Our system is running Windows 7. While there may be a version of LabVIEW for runtime installed on the controller, we suggest

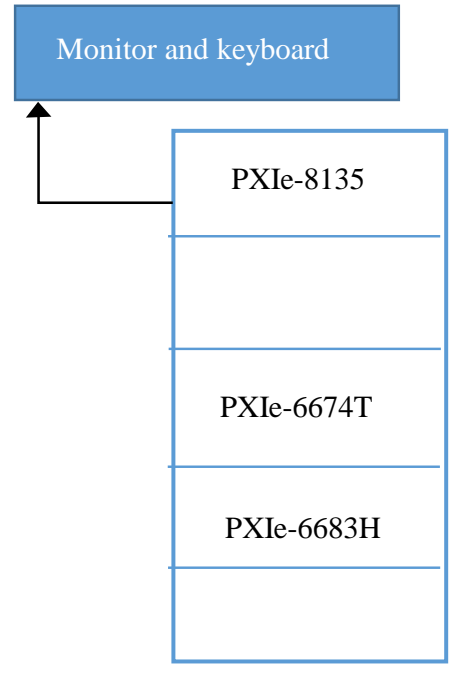

Fig. 7-3 Installation of PXIe6683H and PXIe-6674T into the PXIe-1085 receiver chassis. installing a stand-alone LabVIEW for data collection purposes. For the results shown here, our LabView runs on the 2014 version at 32 bits. After installation, turn off

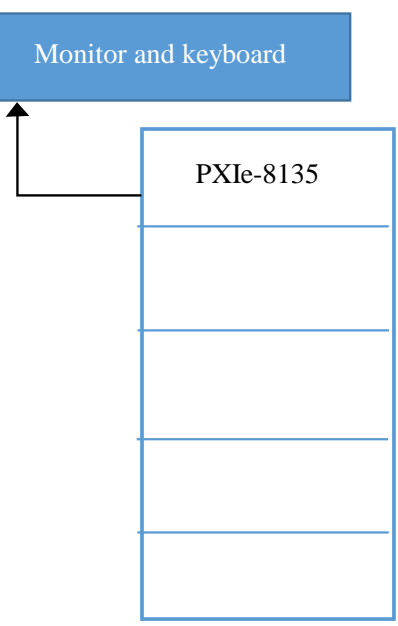

Fig. 7-1 Installation of PXIe-8135 controller into the PXIe-1085 receiver the controller.

Turn on the controller. Next, the NI-Sync drivers (15.0.1 Ni_Sync est) for the PXI-6683H PPS timing synchronization hardware and PXIe-6674T Timing Synchronization modules need to be installed. After the installation, turn off the controller. Next, the PXie-6683H PXIe-6674T can be physically inserted into the PCIe-1085 Receiver Chassis as seen in Fig. 7-3.

\section{PXIe-6674T must be inserted into the dark circle with a square around the circle slot seen on the} PXIe-1085 chassis.

For the NIST system, the PXIe-6674T was inserted into slot 10 and the PXIe-6683H was inserted into slot 11. A double check of the correct installation is done by looking at the NI IMAX and seeing the modules in the software. Next, turn off the entire system.

Turn on the controller. Note that the NI Instrument Design Libraries (http://www.ni.com/download/ni-pxie-5644r5645r5646rinstrument-design-libraries-15.0/5578/en/) must be installed onto the PXIe-8135 controller. Turn off the system. Next, the PXIe-5646R

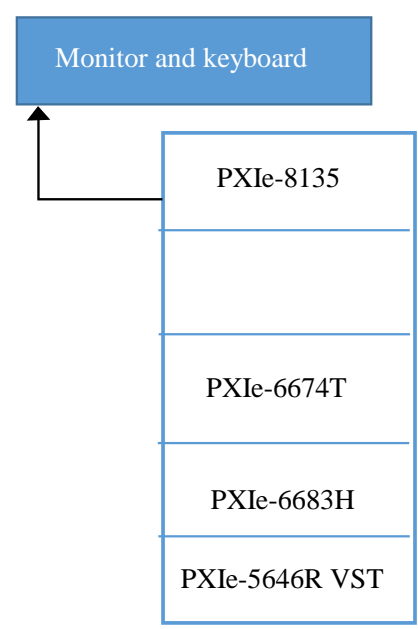

Fig. 7-2 Installation of PXIe-5646R VST into the PXIe-1085 receiver chassis. 
VST module can be physically inserted into the PCIe-1075 Receiver Chassis as seen in Fig. 7-2. Turn on the system, double check using the NI IMAX software that the PXIe-5646R VST module was installed correctly. Turn off the system. Turn on the system put the entire folder contents of VST Channel Sounder-TX or -Rx folder into the NI LabVIEW Instruments. 


\section{Appendix C - Channel Sounder Measurement Software Settings}

The software settings for the TX and RX PXIe-8135 controllers are shown in the below tables.

Table 8-1 Set time reference LabVIEW code.

\begin{tabular}{|l|c|}
\hline \multicolumn{1}{|c|}{ Name } & Input Value \\
\hline Resource Name & PPS Timing \& Sync Module (NI 6683H) \\
\hline Time Reference & IRIGB Dl \\
\hline $\begin{array}{l}\text { Inter-Range Instrumentation Group (IRIG) Parameter: } \\
\text { IRIG Type }\end{array}$ & IO/6683H/PF10 \\
\hline Time Reference Terminal for IRIG and GPS & 0 \\
\hline Time Reference Correction (s) & 10 \\
\hline Clock Resolution (ns) & PPS \\
\hline Time Reference & \multicolumn{1}{|c|}{. } \\
\hline
\end{tabular}

TABLE 8-2 Discipline clock LabVIEW code.

\begin{tabular}{|l|c|}
\hline \multicolumn{1}{|c|}{ Name } & Value \\
\hline System Timing Module & Timing \& Sync Module (NI 6674T) \\
\hline Time Base Synchronization Module & PPS Timing \& Sync Module (NI 6683H) \\
\hline Time Reference & 0 \\
\hline
\end{tabular}

Table 8-3 Rubidium clock model FS725 specifications.

\begin{tabular}{|l|c|}
\hline \multicolumn{1}{|c|}{ Name } & Input Value \\
\hline Phase Noise for 10 MHz & Less Than $130 \mathrm{dBc} / \mathrm{Hz}$ \\
\hline Offset from Carrier Frequency & $10 \mathrm{~Hz}$ \\
\hline $\begin{array}{l}\text { Phase Noise for 10 MHz at a 10 } \mathrm{kHz} \text { Offset from } \\
\text { Carrier Frequency }\end{array}$ & Less Than $155 \mathrm{dBc} / \mathrm{Hz}$ \\
\hline Allan Variance & $2 \times 10^{-11}$ At One Second And \\
& $2 \times 10^{-12}$ At 100 Seconds \\
\hline 1 PPS TTL Output & Less Than 1 Nanosecond of Jitter \\
\hline Rb Clock Long Term Stability of 20 Years Aging & Less Than $0.005 \mathrm{ppm}$ \\
\hline
\end{tabular}




\section{Appendix D - Variability of Sounder Measurements in Manufacturing Facilities Guide}

\subsection{Variability in Path Loss Linear Regression}

The linear regression used to find path gain or path loss provides channel information through the calculation of the slope. The slope of the linear regression provides insight into the severity of path gain or path loss as a function of position for the channel. The linear regression model with one independent variable for path loss vs. distance is shown in Eq. 9.1

$$
\rho=A+B r_{t}
$$

where $\rho$ is the path loss in $\mathrm{dB}, r_{t}$ is the true check point value in meters, $A$ and $B$ are sampled leastsquare estimators. Note that $r_{t}$ is not observed directly. Instead, the distance between the channel sounder's transmitter and receiver, $r$, is observed. Eq. 8.1 becomes

$$
\begin{aligned}
& \rho=A+B r \\
& r=r_{t}+r_{P E},
\end{aligned}
$$

where $r_{P E}$ is the range position error which includes random perturbations. We assume that $r_{P E}$ is distributed as a normal random variable with mean zero and a standard deviation of $\sigma_{r_{P E}}$. The sampled least-square estimators, $A$ and $B$, for the linear regression model can be determined with a known $\sigma_{r_{P E}}[18]$.

\subsection{Range Position Error, $\boldsymbol{r}_{P E}$}

We simulated a range position error using a Monte Carlo simulation for small $x_{P E}$ and $y_{P E}$ surrounding a single $r_{t}$. We use the standard deviation $\sigma_{r_{P E}}$ of the normal distribution to quantify the error $r_{P E}$ for different measured check point true values $r_{t}$ in a NIST Gaithersburg Machine Shop. A variety of standard deviation values were calculated with a Monte Carlo Position Uncertainty algorithm. An example of this algorithm for perturbation in Cartesian $x_{P E} y_{P E}$ is shown in Eqs. 9.4 - 9.6:

$$
\begin{gathered}
x_{P E}= \pm v \cdot t \cdot \varsigma+\frac{1}{2} a \cdot t^{2} \cdot \varsigma, \\
y_{P E}= \pm v \cdot t \cdot \varsigma+\frac{1}{2} a \cdot t^{2} \cdot \varsigma, \\
\quad \sim U(0,1),
\end{gathered}
$$

where $\varsigma$ is a pseudorandom value from a uniform distribution with the open interval of $(0,1), v$ is velocity, $t$ is time, and $a$ is acceleration.

The standard deviation $\sigma_{r_{P E}}=\sqrt{\sigma_{r_{P E}}^{2}}$ [18] is found with (9.7)

$$
\sigma_{r_{P E}}^{2}=\frac{\bar{x}_{P E}^{2} \sigma_{x_{P E}}^{2}+\bar{y}_{P E}^{2} \sigma_{y_{P E}}^{2}+2 \bar{x}_{x_{P E}} \bar{y}_{y_{P E}} \sigma_{x y_{P E}}}{\bar{x}_{P E}^{2}+\bar{y}_{P E}^{2}}
$$


where $\bar{x}_{P E}$ is the mean of the $x_{P E}, \sigma_{x_{P E}}^{2}$ is the variance of $x_{P E}, \bar{y}_{P E}$ is the mean of the $y_{P E}, \sigma_{y_{P E}}^{2}$ is the variance of $y$, and $\sigma_{x y_{P E}}$ is the covariance of $x_{P E} y_{P E}$.

Table 9-1 contains different simulated $\sigma_{r_{P E}}$ values based upon time, velocity, acceleration.

Table 9-1: $\sigma_{r_{P E}}$ versus time, velocity, acceleration.

\begin{tabular}{|l|c|c|c|c|}
\hline & $t(\mathrm{~s})$ & $v(\mathrm{~m} / \mathrm{s})$ & $a\left(\mathrm{~m} / \mathrm{s}^{2}\right)$ & $\sigma_{\boldsymbol{r}_{\boldsymbol{P E}}}$ \\
\hline Stationary & 0 & 0 & 0 & 0 \\
\hline Robot & 3 & 0.7 & 0.5 & 1.4 \\
\hline Human Walking & 3 & 1.4 & 0.75 & 2.6 \\
\hline Human Running & 5 & 10 & 1 & 29 \\
\hline
\end{tabular}

\subsection{Calculation of Uncertainty in A B}

With the assumption that the error in $r_{P E}$ is quantified by $\sigma_{r_{P E}}, B, A$ their uncertainties can be computed with Eqs. 9.8 - 9.12

$$
\begin{gathered}
B=\frac{\sigma_{\rho r}}{\sigma_{r}^{2}-\sigma_{r_{P E}}^{2}}, \\
A=\bar{\rho}-B \bar{r}, \\
B_{\text {Uncert }}=\frac{1}{(M-1)\left(\sigma_{r}^{2}-\sigma_{r_{P E}}^{2}\right)^{2}}\left(\sigma_{r}^{2} s_{v v}+B^{2} \sigma_{r_{P E}}^{2}\right), \\
A_{\text {Uncert }}=(\bar{r})^{2} B_{\text {Uncert }}+\frac{s_{v v}}{M}, \\
s_{v v}=\frac{1}{M-2} \sum_{m=1}^{M}\left[\left(\rho_{m}-\bar{\rho}\right)-\left(r_{m}-\bar{r}\right) B\right]^{2},
\end{gathered}
$$

where $\sigma_{\rho r}$ is the covariance between the path loss $\rho$ the range $r, \sigma_{r}^{2}$ is the variance of the range, $\bar{\rho}$ is the mean of $\rho, \bar{r}$ is the mean of the range, and $M$ is the number of samples. In Table 9-2, the uncertainties for $A$ and $B$ were determined for different $\sigma_{r_{P E}}$. From Table 9-1, when $\sigma_{r_{P E}}$ equals $2.6 \mathrm{~m}$, which corresponds to a human walking, the uncertainty of $B$ was 0.054 . Based upon these values, the channel sounder platform of a human walking lies within the two sigma bounds of the bounds of the Stationary case. Fig. 9-1 shows the standard path loss versus range with the uncertainties introduced by $\sigma_{r_{P E}}$ for a human walking as compared to path loss data collected for the Stationary case.

Table 9-2: B, uncertainty B, A, uncertainty A results

\begin{tabular}{|l|c|c|c|c|}
\hline & $B(d B / m)$ & Uncertainty $B$ & $A(d B)$ & Uncertainty $A$ \\
\hline Stationary & 3 & 0.0036 & 21 & 0.62 \\
\hline Robot & 4.9 & 0.026 & -3 & 4.4 \\
\hline Human Walking & 4.9 & 0.054 & -3 & 9.2 \\
\hline Human Running & 4.9 & 130 & -3 & 2300 \\
\hline
\end{tabular}




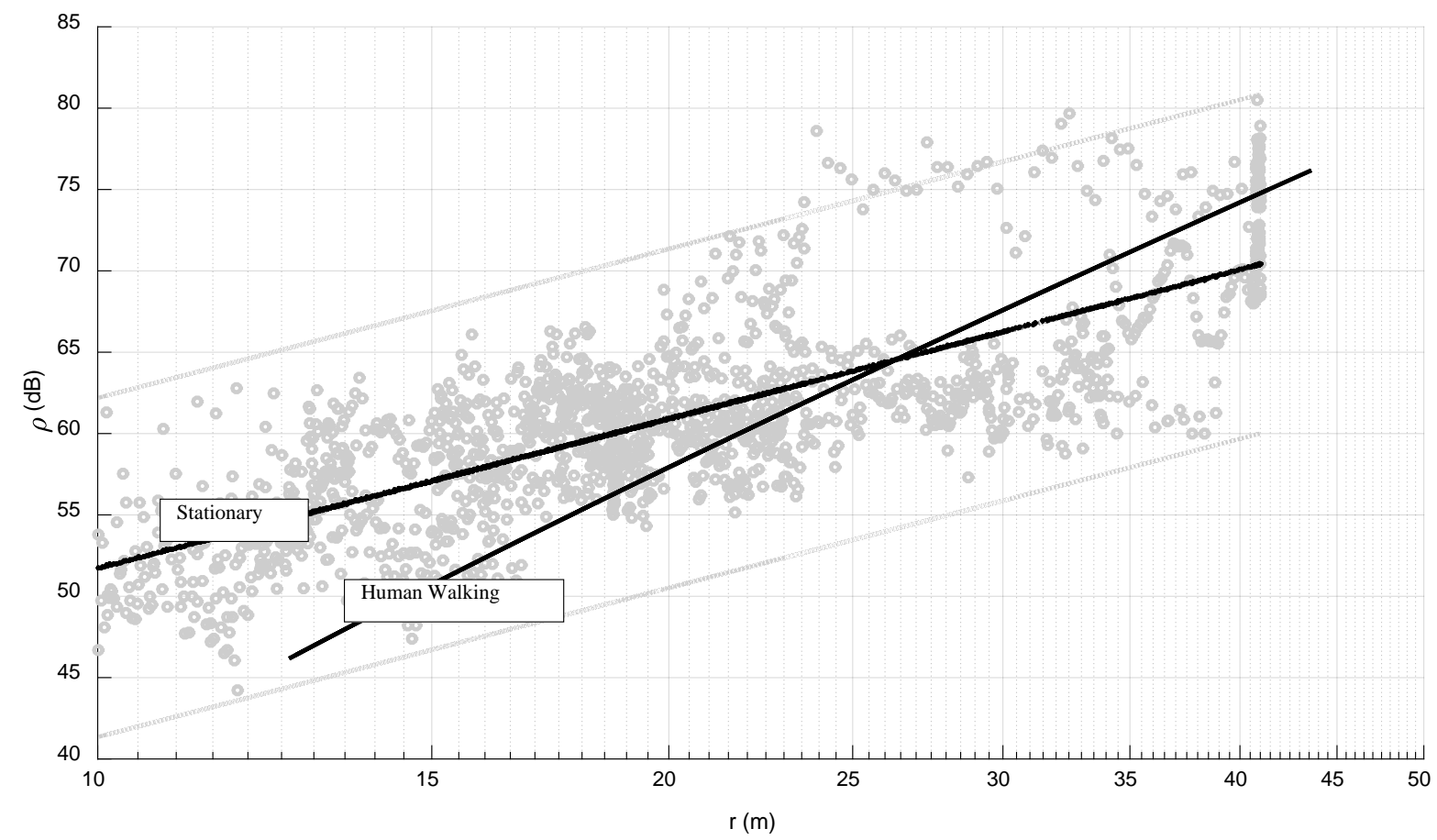

Fig. 9-1 Path loss versus range with uncertainties for a human walking.

$B$, $A$, and their uncertainties provide insight into the required accuracy between physical location path loss, when choosing between a robot or the human walking approach for different levels of accuracy in check point position. 


\section{References}

[1] R. Candell , Remley, J. T. Quimby, D. R. Novotny, A. E. Curtin, P. B. Papazian, G. H. Koepke, J. E. Diener, M. T. Hany, "Industrial Wireless Systems: Radio Propagation Measurements" Technical Note (NIST TN) 1951, 125 pp., (30-Jan-2017)

[2] D. Novotny, A. Curtin, J. Quimby, K. Remley, P. Papazian, R. Candell, “A Tetherless, Absolute-Time Channel Sounder; Processing Results for a Complex Environment,” AMTA, 2016, pg. 1-6

[3] TECOM Industries Inc. Broad Omnidirectional, Slant Linear, Biconical Antenna [Online]. Available: http://www.tecom-ind.com/files/1/5361632ea18b8-WebOMA0305004RevA.pdf

[4] ETS-LINDGREN. 3115 Double-Ridged Guide Antenna [Online]. Available: http://www.etslindgren.com $/ 3115$

[5] Seibersdorf Laboratories. Precision Omnidirectional Dipoles 1 - $18 \mathrm{GHz}$ [Online]. Available: https://rf.seibersdorf-laboratories.at/products-services/antennas/pod

[6] A. Molisch, “Wireless Communications,” J. Wiley \& Sons Ltd., 2011

[7] P. Papazian, K. Remley, K. Gentile, N. Golmie, "Radio Channel Sounders for Modeling Mobile Communications at $28 \mathrm{GHz}, 60 \mathrm{GHz}, 83 \mathrm{GHz}$,” IEEE Millimeter Waves, 2015 Global Symposium, 2015, pg. 1-3

[8] R. Sun, D. Williams, J. Quimby, K. Remley, D. Novotny, C. Gentile, P. Papazian, A. Curtin, "Power Delay Profile Calibration for Correlation-Based Channel Sounders", in progress

[9] P. Papazian and J. Lemmon, "Radio Channel Impulse Response Measurement and Analysis," NTIA Technical Report TR-11-476, May 2011

[10] NIST Networked Control System Group Website, http://doi.org/10.18434/T44S3N.

[11] S. Ryan and L. Porth, "A Tutorial on the Piecewise Regression Approach Applied to Bedload Transport Data”, General Technical Report RMRS-GTR-189, May 2007

[12] R. Marhefka. "Numerical Electromagnetic Code - Basic Scattering Code (NEC-BSC) "Version 4.6, User’s Manual, Parts 1- 7, 2016

[13] D. Sexton, M. Mahony, M. Lapinski, and J. Werb, "Radio Channel Quality in Industrial Wireless Sensor Networks””' IEEE Sensors for Industry Conference, 2005, pg. 1-7

[14] T. Rappaport, "Characterization of UHF Multipath Radio Channels in Factory Buildings," IEEE Trans. Antennas and Prop., Vol 37, NO. 8, August 1989 
[15] S. Sun, T. Rappaport, T. Thomas, A. Ghosh, H. Nguyen, . I. Kovac, I. Rodriguez, O. Koymen, and A. Partyka, "Investigation of Prediction Accuracy, Sensitivity, and Parameter Stability of Large-Scale Propagation Path Loss Models for 5G Wireless Communications,” IEEE Trans. Antennas and Prop., Vol 65, NO. 5, May 2016

[16] E. Tanghe, W. Joseph, L. Verloock, L. Mertens, H. Capoen, K. Herwegen, W. Vantomme, "The Industrial Indoor Channel: large-Scale and Temporal Fading at 900, 2400, and $5200 \mathrm{MHz}$," IEEE Trans. Wireless Communication, Vol. 7, No. 7, July 2008, pg. 2740-2750

[17] S. Salous, Radio Propagation measurement Channel Modeling, Ed. Chichester, U. K., 2013

[18] W. Fuller, Measurement Error Models, John Wiley \& Sons, Inc, 1987 